\title{
2017 Guidelines of the American Thyroid Association for the Diagnosis and Management of Thyroid Disease During Pregnancy and the Postpartum
}

\author{
Erik K. Alexander,, ${ }^{1, *}$ Elizabeth N. Pearce, ${ }^{2, *}$ Gregory A. Brent, ${ }^{3}$ Rosalind S. Brown, ${ }^{4}$ Herbert Chen, \\ Chrysoula Dosiou, William A. Grobman, ${ }^{7}$ Peter Laurberg, ${ }^{8, \uparrow}$ John H. Lazarus, ${ }^{9}$ Susan J. Mandel, ${ }^{10}$ \\ Robin P. Peeters, ${ }^{11}$ and Scott Sullivan ${ }^{12}$
}

Background: Thyroid disease in pregnancy is a common clinical problem. Since the guidelines for the management of these disorders by the American Thyroid Association (ATA) were first published in 2011, significant clinical and scientific advances have occurred in the field. The aim of these guidelines is to inform clinicians, patients, researchers, and health policy makers on published evidence relating to the diagnosis and management of thyroid disease in women during pregnancy, preconception, and the postpartum period.

Methods: The specific clinical questions addressed in these guidelines were based on prior versions of the guidelines, stakeholder input, and input of task force members. Task force panel members were educated on knowledge synthesis methods, including electronic database searching, review and selection of relevant citations, and critical appraisal of selected studies. Published English language articles were eligible for inclusion. The American College of Physicians Guideline Grading System was used for critical appraisal of evidence and grading strength of recommendations. The guideline task force had complete editorial independence from the ATA. Competing interests of guideline task force members were regularly updated, managed, and communicated to the ATA and task force members.

Results: The revised guidelines for the management of thyroid disease in pregnancy include recommendations regarding the interpretation of thyroid function tests in pregnancy, iodine nutrition, thyroid autoantibodies and pregnancy complications, thyroid considerations in infertile women, hypothyroidism in pregnancy, thyrotoxicosis in pregnancy, thyroid nodules and cancer in pregnant women, fetal and neonatal considerations, thyroid disease and lactation, screening for thyroid dysfunction in pregnancy, and directions for future research.

Conclusions: We have developed evidence-based recommendations to inform clinical decision-making in the management of thyroid disease in pregnant and postpartum women. While all care must be individualized, such recommendations provide, in our opinion, optimal care paradigms for patients with these disorders.

Keywords: pregnancy, thyroid and pregnancy, thyroid function tests, postpartum thyroiditis

\footnotetext{
${ }^{1}$ Division of Endocrinology, Diabetes and Hypertension, Brigham and Women's Hospital and Harvard Medical School, Boston, Massachusetts.

${ }^{2}$ Section of Endocrinology, Diabetes, and Nutrition, Boston University School of Medicine, Boston, Massachusetts.

${ }^{3}$ Department of Medicine, VA Greater Los Angeles Healthcare System and David Geffen School of Medicine at UCLA, Los Angeles, California.

${ }^{4}$ Division of Endocrinology, Boston Children's Hospital, Harvard Medical School, Boston, Massachusetts.

${ }^{5}$ Department of Surgery, University of Alabama at Birmingham, Birmingham, Alabama.

${ }^{6}$ Division of Endocrinology, Stanford University School of Medicine, Stanford, California.

${ }^{7}$ Department of Obstetrics and Gynecology, Northwestern University, Chicago, Illinois.

${ }^{8}$ Departments of Endocrinology \& Clinical Medicine, Aalborg University Hospital, Aalborg, Denmark.

${ }_{9}^{9}$ Institute of Molecular Medicine, Cardiff University, Cardiff, United Kingdom.

${ }^{10}$ Division of Endocrinology, Diabetes and Metabolism, Perelman School of Medicine, University of Pennsylvania, Philadelphia, Pennsylvania.

${ }^{11}$ Department of Internal Medicine and Rotterdam Thyroid Center, Erasmus Medical Center, Rotterdam, The Netherlands.

${ }^{12}$ Department of Obstetrics and Gynecology, Medical University of South Carolina, Charleston, South Carolina.

*Co-chairpersons: Erik K. Alexander and Elizabeth N. Pearce. Excepting the co-chairpersons, the authors are listed in alphabetical order and were appointed by the ATA to independently formulate the content of this manuscript. None of the scientific or medical content of the manuscript was dictated by the ATA.

${ }^{\dagger}$ Deceased.
} 


\section{DEDICATION}

These guidelines are dedicated to the memory of Dr. Peter Laurberg, our friend and colleague, who died tragically during their preparation.

\section{INTRODUCTION}

$\mathbf{P}$ REGNANCY HAS A PROFOUND IMPACT on the thyroid gland and its function. During pregnancy, the thyroid gland increases in size by $10 \%$ in iodine replete countries but by $20 \%$ to $40 \%$ in areas of iodine deficiency. Production of the thyroid hormones, thyroxine (T4), and triiodothyronine (T3), increases by nearly $50 \%$, in conjunction with a separate $50 \%$ increase in the daily iodine requirement. These physiological changes happen seamlessly in healthy women, but thyroid dysfunction can occur in many pregnant women because of pathologic processes. Furthermore, other thyroid illnesses such as nodular disease and thyroid cancer are occasionally detected during pregnancy and may require treatment. Together, the burden of thyroid disease affecting women, either before, during, or directly after pregnancy, is substantial.

For these reasons thyroid function is frequently assessed during the gestation period. However, accurate assessment of maternal (and fetal) thyroid function during pregnancy remains difficult, and interpretation of laboratory testing differs from the nonpregnant patient. Placental human chorionic gonadotropin (hCG) stimulates thyroid hormone secretion, often decreasing maternal thyrotropin (TSH) concentrations, especially in early pregnancy. But while such transiently suppressed maternal TSH concentrations are often observed and deemed safe, defining the upper reference limit for serum TSH in this population has remained controversial. Furthermore, up to $18 \%$ of all pregnant women are thyroid peroxidase antibody (TPOAb) or thyroglobulin antibody (TgAb) positive. Increasingly, data suggest that TPOAb positivity adversely modulates the impact of maternal thyroid status (especially hypothyroidism) on the pregnancy and the developing fetus. Thyroid antibody positivity separately increases the risk of thyroid dysfunction following delivery and during the postpartum period.

Studies have recently questioned the optimal treatment of hyperthyroidism during pregnancy. Clinical management of patients with Graves' disease (GD) is challenged by the understanding that maternal antibodies as well as antithyroid medication may differentially affect maternal and fetal thyroid function. Reports have also detailed the potential teratogenic effects of the antithyroid medications methimazole (MMI) and propylthiouracil (PTU). But while mild hyperthyroidism appears safe for the mother and fetus, moderate to severe hyperthyroidism can prove dangerous. Thus, when and how to treat affected mothers during pregnancy remains an important clinical question. Following delivery, mothers often choose to breastfeed. Separate questions surround the optimal approach to the treatment of hypo- and hyperthyroidism while lactating.

Given the prevalence and potential dangers detailed above, many have suggested universally evaluating thyroid function in all women either before or during pregnancy. Such a screening mandate, however, must take the cost, effectiveness, and practical nature of any such approach into account. To date, studies evaluating this question appear to demonstrate mixed conclusions. Several ongoing investigations will shed further light on this difficult question.

Given the complexity surrounding thyroid physiology and thyroid illness during pregnancy and the postpartum period, how and when to evaluate for thyroid dysfunction and how and if to treat thyroid illness during this period remain challenging.

In 2011, the American Thyroid Association (ATA) first published guidelines on the diagnosis and management of thyroid disease during pregnancy and postpartum (1). There has been a substantial amount of new literature in this area since that publication.

It is in this context that the ATA charged a task force to develop revised clinical guidelines on the diagnosis and treatment of thyroid disease during pregnancy and the postpartum period. The task force consisted of both national and international experts in the field of thyroid disease and pregnancy and included representatives from the ATA, the European Thyroid Association (ETA), the American College of Obstetricians and Gynecologists, the Society for Maternal Fetal Medicine, and the Iodine Global Network. In addition to evidence-based updates of traditional content areas, the task force also sought to expand the prior document to address topics such as thyroid disease during lactation, the treatment of thyroid illness in infertile women and those undergoing assisted reproductive techniques (ART), as well as the approach to thyroid disease in the newborn.

The literature review for each section included an analysis of all primary studies in the area published since 1990 and selective review of the primary literature published prior to 1990 that was seminal in the field. In the past 25 years, there have been a number of recommendations and guideline statements relating to aspects of thyroid disease and pregnancy. In deriving the present guidelines, the task force conducted a new and comprehensive analysis of the primary literature and reformulated all clinical recommendations. In doing so, this document represents the best effort to create a useful, practical, and accurate guideline designed to help the practicing clinician, while also stimulating future research and discovery into this important and complex arena.

\section{METHODS}

ATA Thyroid Disease in Pregnancy guidelines were previously published in 2011 (1). Because of the rapid growth of the literature relating to this topic, plans for revising the guidelines within $\sim 4-5$ years of publication were made at the inception of the project. Task force chairs were appointed by the ATA President with approval of the Board. A task force of specialists with complementary expertise (adult and pediatric endocrinology, obstetrics, maternal-fetal medicine, endocrine surgery, iodine nutrition, and epidemiology) was appointed. In order to have broad specialty and geographic representation, as well as fresh perspectives, approximately one third of the task force is to be replaced for each iteration of the guidelines, as per ATA policy. In accordance with current ATA policies, the American College of Physicians Grading System was adopted for use in these guidelines (Tables 1 and 2) (2).

Prior to initiating the reviews, all task force members were provided written and verbal group advice on conducting electronic literature searches, critical appraisal of articles, 
Table 1. Recommendations (For Therapeutic Interventions) Based on Strength of Evidence

\begin{tabular}{|c|c|c|}
\hline $\begin{array}{l}\text { Recommendation } \\
\text { and evidence quality }\end{array}$ & $\begin{array}{l}\text { Description } \\
\text { of supporting evidence }\end{array}$ & Interpretation \\
\hline \multicolumn{3}{|l|}{ Strong recommendation } \\
\hline High-quality evidence & $\begin{array}{l}\text { RCT without important limitations } \\
\text { or overwhelming evidence from } \\
\text { observational studies }\end{array}$ & $\begin{array}{l}\text { Can apply to most patients in most } \\
\text { circumstances without reservation }\end{array}$ \\
\hline $\begin{array}{l}\text { Moderate-quality } \\
\text { evidence }\end{array}$ & $\begin{array}{l}\text { RCT with important limitations or strong } \\
\text { evidence from observational studies }\end{array}$ & $\begin{array}{l}\text { Can apply to most patients in most } \\
\text { circumstances without reservation }\end{array}$ \\
\hline Low-quality evidence & Observational studies/case studies & $\begin{array}{l}\text { May change when higher-quality } \\
\text { evidence becomes available }\end{array}$ \\
\hline \multicolumn{3}{|l|}{ Weak recommendation } \\
\hline High-quality evidence & $\begin{array}{l}\text { RCT without important limitations } \\
\text { or overwhelming evidence from } \\
\text { observational studies }\end{array}$ & $\begin{array}{l}\text { Best action may differ based on } \\
\text { circumstances or patients' values }\end{array}$ \\
\hline $\begin{array}{l}\text { Moderate-quality } \\
\text { evidence }\end{array}$ & $\begin{array}{l}\text { RCT with important limitations or strong } \\
\text { evidence from observational studies }\end{array}$ & $\begin{array}{l}\text { Best action may differ based on } \\
\text { circumstances or patients' values }\end{array}$ \\
\hline Low-quality evidence & Observational studies/case studies & Other alternatives may be equally reasonable \\
\hline Insufficient & $\begin{array}{l}\text { Evidence is conflicting, of poor quality, } \\
\text { or lacking }\end{array}$ & $\begin{array}{l}\text { Insufficient evidence to recommend } \\
\text { for or against }\end{array}$ \\
\hline
\end{tabular}

${ }^{\text {a }}$ This description of supporting evidence refers to therapy, therapeutic strategy, or prevention studies. The description of supporting evidence is different for diagnostic accuracy studies.

$\mathrm{RCT}$, randomized controlled trial.

Table 2. Recommendations (for Diagnostic Interventions) Based on Strength of Evidence

\begin{tabular}{|c|c|c|}
\hline $\begin{array}{l}\text { Recommendation } \\
\text { and evidence quality }\end{array}$ & $\begin{array}{l}\text { Methodologic quality } \\
\text { of supporting evidence }\end{array}$ & Interpretation \\
\hline \multicolumn{3}{|c|}{ Strong recommendation } \\
\hline $\begin{array}{l}\text { High-quality } \\
\text { evidence }\end{array}$ & $\begin{array}{l}\text { Evidence from one or more well-designed nonrandomized } \\
\text { diagnostic accuracy studies (i.e., observational-- } \\
\text { cross-sectional or cohort) or systematic reviews/ } \\
\text { meta-analyses of such observational studies } \\
\text { (with no concern about internal validity or external } \\
\text { generalizability of the results). }\end{array}$ & $\begin{array}{l}\text { Implies the test can be offered to most } \\
\text { patients in most applicable } \\
\text { circumstances without reservation. }\end{array}$ \\
\hline $\begin{array}{l}\text { Moderate-quality } \\
\text { evidence }\end{array}$ & $\begin{array}{l}\text { Evidence from nonrandomized diagnostic accuracy } \\
\text { studies (cross-sectional or cohort), with one or } \\
\text { more possible limitations causing minor concern } \\
\text { about internal validity or external generalizability } \\
\text { of the results. }\end{array}$ & $\begin{array}{l}\text { Implies the test can be offered to most } \\
\text { patients in most applicable } \\
\text { circumstances without reservation. }\end{array}$ \\
\hline $\begin{array}{l}\text { Low-quality } \\
\text { evidence }\end{array}$ & $\begin{array}{l}\text { Evidence from nonrandomized diagnostic accuracy } \\
\text { studies with one or more important limitations } \\
\text { causing serious concern about internal validity } \\
\text { or external generalizability of the results. }\end{array}$ & $\begin{array}{l}\text { Implies the test can be offered to most } \\
\text { patients in most applicable } \\
\text { circumstances, but the utilization } \\
\text { of the test may change when higher- } \\
\text { quality evidence becomes available. }\end{array}$ \\
\hline \multicolumn{3}{|l|}{ Weak recommendation } \\
\hline $\begin{array}{l}\text { High-quality } \\
\text { evidence }\end{array}$ & $\begin{array}{l}\text { Evidence from one or more well-designed } \\
\text { nonrandomized diagnostic accuracy studies } \\
\text { (i.e., observational-cross-sectional or cohort) } \\
\text { or systematic reviews/meta-analyses of such } \\
\text { observational studies (with no concern about } \\
\text { internal validity or external generalizability } \\
\text { of the results). }\end{array}$ & $\begin{array}{l}\text { The degree to which the diagnostic test } \\
\text { is seriously considered may differ } \\
\text { depending on circumstances or patients' } \\
\text { or societal values. }\end{array}$ \\
\hline $\begin{array}{l}\text { Moderate-quality } \\
\text { evidence }\end{array}$ & $\begin{array}{l}\text { Evidence from nonrandomized diagnostic } \\
\text { accuracy studies (cross-sectional or cohort), with } \\
\text { one or more possible limitations causing minor } \\
\text { concern about internal validity or external } \\
\text { generalizability of the results. }\end{array}$ & $\begin{array}{l}\text { The degree to which the diagnostic test } \\
\text { is seriously considered may differ } \\
\text { depending on individual patients'/ } \\
\text { practice circumstances or patients' } \\
\text { or societal values. }\end{array}$ \\
\hline $\begin{array}{l}\text { Low-quality } \\
\text { evidence }\end{array}$ & $\begin{array}{l}\text { Evidence from nonrandomized diagnostic accuracy } \\
\text { studies with one or more important limitations } \\
\text { causing serious concern about internal validity } \\
\text { or external generalizability of the results. }\end{array}$ & $\begin{array}{l}\text { Alternative options may be equally } \\
\text { reasonable. }\end{array}$ \\
\hline Insufficient & $\begin{array}{l}\text { Evidence may be of such poor quality, conflicting, } \\
\text { lacking (i.e., studies not done), or not externally } \\
\text { generalizable to the target clinical population such } \\
\text { that the estimate of the true effect of the test } \\
\text { is uncertain and does not permit a reasonable } \\
\text { conclusion to be made. }\end{array}$ & $\begin{array}{l}\text { Insufficient evidence exists to recommend } \\
\text { for or against routinely offering the } \\
\text { diagnostic test. }\end{array}$ \\
\hline
\end{tabular}


and rationale for formulating strength of recommendations. Standardized data collection forms were used by all reviewers. For each question, a primary reviewer performed a literature search, appraised relevant literature, and generated recommendations, accompanying text, and a relevant bibliography. This information was then reviewed by both chairs, revised as needed, and presented for review by the entire panel. Feedback and suggestions for revisions from the Chairs and panel members were obtained via e-mail, regularly scheduled teleconferences, and face-to-face meetings. Once the manuscript was drafted, all suggestions for revisions were regularly reviewed by the entire panel in the form of a tracked changes draft manuscript and teleconferences. The draft document continued to be revised until no suggestions for further revisions were requested by any panel members. Thus, general consensus on acceptability of recommendations and manuscript text was achieved, with the fundamental understanding that not all recommendations may be feasible in all practice settings and adaptation of the guideline recommendations to individual care may be needed.

Formal stakeholder input in development of these guidelines was sought from ATA membership via an online survey distributed in October 2014. We also reviewed any letters, editorials, or reviews of the 2011 iteration of these guidelines (1) that were collected by the current chairs of the task force. Prepublication verbal feedback on some of the key guideline recommendations was received at a formal Satellite Symposium held in conjunction with the Endocrine Society meeting in Boston on March 31, 2016. The guidelines were then provided to the ATA membership for review and comments over a 2 -week period. The guideline manuscript was next reviewed and approved by the ATA Board of Directors. Feedback and suggestions were formally discussed by the panel, and revisions were made to the manuscript prior to journal submission. The organization of management guideline recommendations is shown in Table 3.

TABle 3. Organization of the TASK Force's RECOMMENDATIONS

\begin{tabular}{llc}
\hline Location key & \multicolumn{1}{c}{ Description } & Page \\
\hline I & Introduction & 316 \\
II & Methods & 316 \\
III & Thyroid Function Testing and & 318 \\
& $\quad$ Pregnancy & 321 \\
IV & Iodine Status and Nutrition \\
V & Thyroid Autoantibodies and & 324 \\
& $\quad$ Pregnancy Complications \\
VI & The Impact of Thyroid Illness \\
& $\quad$ upon Infertility and Assisted \\
& $\quad$ Reproduction & 328 \\
VII & Hypothyroidism and Pregnancy \\
VIII & Thyrotoxicosis in Pregnancy \\
IX & Thyroid Nodules and Thyroid & 331 \\
& $\quad$ Cancer During Pregnancy \\
X & Fetal and Neonatal Considerations & 343 \\
XI & Thyroid Disease and Lactation \\
XII & Postpartum Thyroiditis & 356 \\
XIII & Screening for Thyroid Dysfunction & 359 \\
& $\quad$ Before or During Pregnancy \\
XIV & Future Research Directions \\
\hline
\end{tabular}

The medical opinions expressed here are those of the authors, and the task force had complete editorial independence from the ATA in writing the guidelines. No funding was received by individual task force members from the ATA or industry for work on these guidelines. Competing interests of all task force members were reviewed at inception of the group, yearly, and upon completion of the guidelines and are included with this document.

These Guidelines were approved by the Board of the ATA.

\section{THYROID FUNCTION TESTING AND PREGNANCY \\ Question 1: How do thyroid function tests change during pregnancy?}

Normal pregnancy is associated with an increase in renal iodine excretion, an increase in thyroxine binding proteins, an increase in thyroid hormone production, and thyroid stimulatory effects of hCG. All of these factors influence thyroid function tests in the pregnant patient. The healthy thyroid adapts to these alterations through changes in thyroid hormone metabolism, iodine uptake, and the regulation of the hypothalamic-pituitary-thyroid axis $(3,4)$. The thyroid function tests of healthy pregnant women, therefore, differ from those of healthy nonpregnant women. Furthermore, the reference ranges for the most widely applied tests, TSH and free thyroxine (FT4), may vary significantly in different populations.

Following conception, circulating thyroxine binding globulin (TBG) and total T4 (TT4) concentrations increase by week 7 of gestation and reach a peak by approximately week 16 of gestation (5). These concentrations then remain high until delivery. In the first trimester, maternal hCG directly stimulates the TSH receptor, increasing thyroid hormone production and resulting in a subsequent reduction in serum TSH concentration (4,6). Therefore, during pregnancy, women have lower serum TSH concentrations than before pregnancy, and a TSH below the nonpregnant lower limit of $0.4 \mathrm{mU} / \mathrm{L}$ is observed in as many as $15 \%$ of healthy women during the first trimester of pregnancy $(7,8)$. In Japan, a suppressed TSH less than $0.6 \mathrm{mU} / \mathrm{L}$ is similarly frequently observed during the first trimester of pregnancy (9). The fraction of women with a suppressed TSH falls to about $10 \%$ in the second trimester, and 5\% in the third trimester (4).

Measurement of FT4 concentration by automated immunoassays results in a significant and assay dependent reduction in the measured serum FT4 concentrations in the third trimester, even though direct measurement of free T4 by more precise methods does not show a similar degree of reduction (4,6,7). Automated immunoassays for FT4, which are employed in most clinical laboratories, are complicated in pregnant women by the increase in TBG and decrease in albumin concentrations $(10,11)$. Other methods of direct measurement, such as measurement by equilibrium dialysis, ultrafiltration, or liquid chromatography/tandem mass spectrometry (LC/MS/MS) are less influenced by the pregnancyassociated changes in serum proteins but are significantly more expensive and less widely available. Thus, the automated immunoassays used for serum FT4 analysis are still widely used, but the important considerations discussed above must be noted. The use of population-based, trimester-specific reference ranges remains the best way to handle this issue. 


\section{Question 2: What is the normal reference range for serum TSH concentrations in each trimester of pregnancy?}

A downward shift of the TSH reference range occurs during pregnancy, with a reduction in both the lower (decreased by about $0.1-0.2 \mathrm{mU} / \mathrm{L}$ ) and the upper limit of maternal TSH (decreased by about $0.5-1.0 \mathrm{mU} / \mathrm{L}$ ), relative to the typical nonpregnant $\mathrm{TSH}$ reference range. The largest decrease in serum TSH is observed during the first trimester because of elevated levels of serum hCG directly stimulating the TSH receptor and thereby increasing thyroid hormone production (Table 4). Thereafter, serum TSH and its reference range gradually rise in the second and third trimesters, but nonetheless remain lower than in nonpregnant women $(12,13)$. Since hCG concentrations are higher in multiple pregnancies than in singleton pregnancies, the downward shift in the TSH reference interval is greater in twin pregnancies (11). In a study of 63 women with hCG concentrations $>200,000 \mathrm{IU} / \mathrm{L}$, TSH was suppressed $(\leq 0.2 \mathrm{mU} / \mathrm{L})$ in $67 \%$ of women and in $100 \%$ of women if hCG concentrations were $>400,000$ IU/L (14). Serum TSH reference range determinations should take into account iodine intake, TPO positivity, and according to some studies, body mass index (BMI).

Although the downward shift in TSH reference ranges is seen in essentially all populations, the extent of this reduction varies significantly between different racial and ethnic groups. Initial studies of pregnant women in the United States and Europe first led to recommendations for a TSH upper reference limit of $2.5 \mathrm{mU} / \mathrm{L}$ in the first trimester and $3.0 \mathrm{mU} /$ $\mathrm{L}$ in the second and third trimesters $(1,15)$. However, more recent studies in pregnant women in Asia, India, and the Netherlands, have demonstrated only a modest reduction in the upper reference limit (16-20). A study of 4800 pregnant women in China recently showed that the downward shift in the $\mathrm{TSH}$ reference range occurred at weeks 7-12, but the upper reference limit was only reduced from 5.31 to $4.34 \mathrm{mU} /$ L (17). Separate data from a recent prospective intervention trial in the United States support this finding (21). Analysis of the TSH and FT4 "set-point" in pregnant women showed that reductions in FT4 were observed only when the serum TSH was greater than $4.8 \mathrm{mU} / \mathrm{L}$. Similar studies of pregnant women in India and Korea show a modest reduction in the first-trimester upper TSH limit of $0.51 .0 \mathrm{mU} / \mathrm{L}$. In some cases, this was not statistically different from the nonpregnant state $(18,20)$. Thus, the current evidence supports only a slight downward shift in the upper reference range of TSH occurring in the latter first trimester of pregnancy, typically not seen prior to week 7 (17).

A reduction in the lower TSH reference range is observed during pregnancy in almost all studies. In a small percentage of women, TSH can be undetectable $(<0.01 \mathrm{mU} / \mathrm{L})$, and yet still represent a normal pregnancy. In addressing the clinical importance of a reduced serum TSH during pregnancy, it is important to note that subclinical hyperthyroidism has not been associated with adverse pregnancy outcomes. Therefore, a maternal TSH concentration that is low but detectable is likely not clinically significant (22). TSH ranges have been shown to vary slightly depending on different methods of analysis, although this variation is not clinically significant (23). One approach to reducing this variability is to use the Multiple of Medians calculation to compare values between assays. This calculation divides an individual value by the population median (24). The resulting value is not influenced by the differences between assays (24).

Significant geographic and ethnic diversity exist in TSH concentrations during pregnancy, as shown in Table 4 . The task force recognizes the limited availability of trimesterspecific reference ranges calculated for most ethnic and racial populations with adequate iodine intake who are free of thyroid autoantibodies. Nonetheless, to provide guidance to all patients and clinicians, the panel recommends use of the following trimester-specific ranges and cutoffs when local assessments are not available. In the first trimester, the lower reference range of TSH can be reduced by approximately $0.4 \mathrm{mU} / \mathrm{L}$, while the upper reference range is reduced by approximately $0.5 \mathrm{mU} / \mathrm{L}$. For the typical patient in early pregnancy, this corresponds to a TSH upper reference limit of $4.0 \mathrm{mU} / \mathrm{L}$. This reference limit should generally be applied beginning with the late first trimester, weeks 7-12, with a gradual return towards the nonpregnant range in the second and third trimesters. For specific recommendations regarding the diagnosis and treatment of maternal hypothyroidism, see Section VII.

\section{- RECOMMENDATION 1}

When possible, population-based trimester-specific reference ranges for serum TSH should be defined through assessment of local population data representative of a health care provider's practice. Reference range determinations should only include pregnant women with no known thyroid disease, optimal iodine intake, and negative TPOAb status.

\section{Strong recommendation, moderate-quality evidence.}

\section{Question 3: What is the optimal method to assess serum T4 concentration during pregnancy?}

Unbound T4 represents only about $0.03 \%$ of serum TT4 content. Importantly, only FT4 is available for tissue uptake, with the remainder of $\mathrm{T} 4$ bound to serum proteins, primarily TBG. Serum TT4 concentrations are measured in the nanomolar range, while FT4 concentrations are measured in the picomolar range. In part because of this, measuring FT4 in the presence of high concentrations of bound $\mathrm{T} 4$ has proved to be challenging. This challenge is especially true in conditions where binding-protein levels are altered, such as pregnancy.

Equilibrium dialysis and ultrafiltration are used for physical separation of serum FT4 from bound T4 prior to analysis of the dialysate or ultrafiltrate. While they are theoretically not influenced by changes in binding proteins and heterophilic antibodies, assays based on classical equilibrium dialysis or ultrafiltration are laborious, time-consuming, expensive, and not widely available.

As noted above, FT4 measurement performed by indirect analog immunoassays is used by the majority of clinical laboratories, largely because of its ability to be quickly performed on automated platforms. Unfortunately, this approach is prone to inaccuracy in the setting of pregnancy because of disruption of the original equilibrium - a process dependent upon dilution, temperature, buffer composition, affinity, and the concentration of the T4 antibody reagent and the T4binding capacity within the serum sample (25). High protein concentrations in serum samples tend to result in higher FT4 


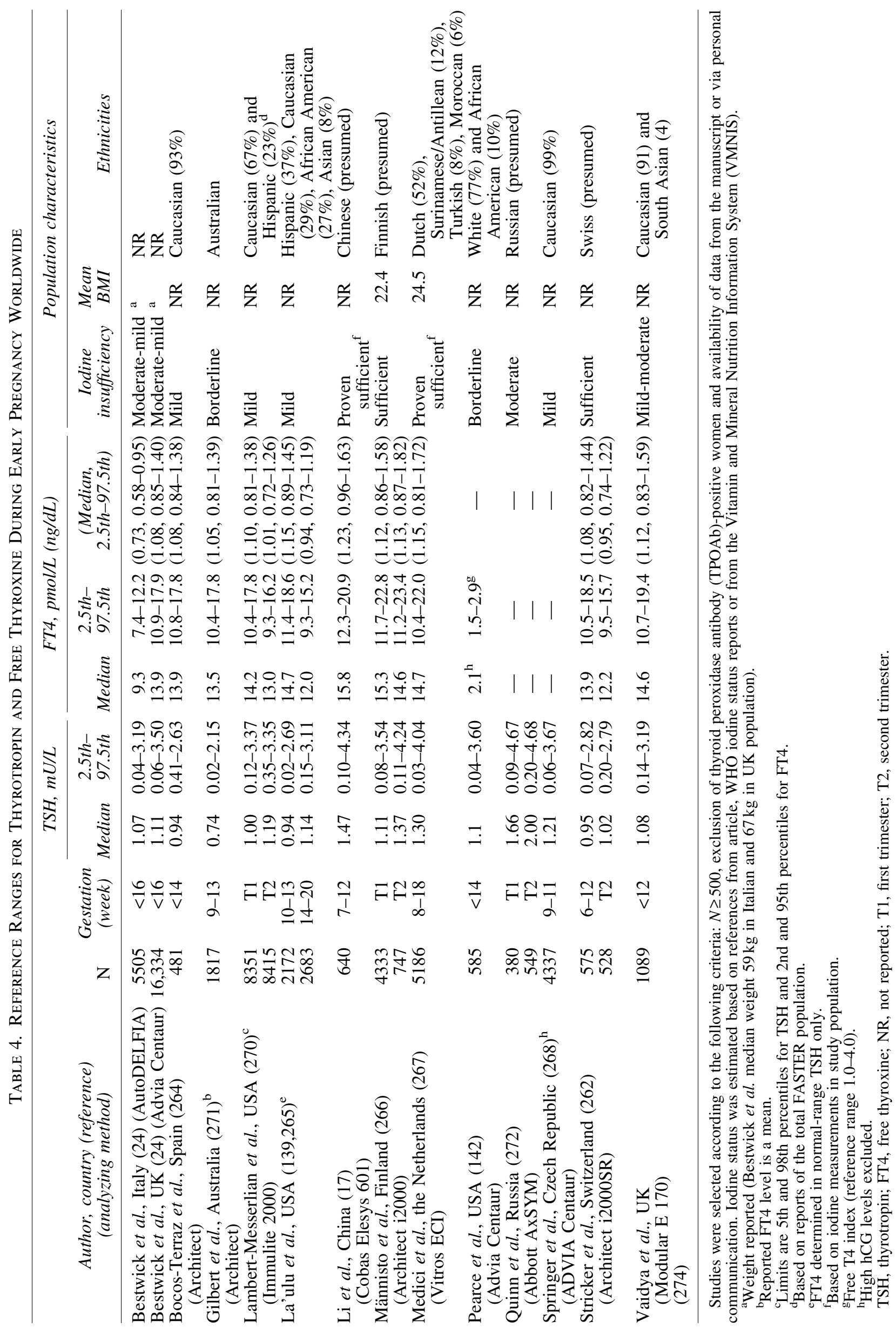


values, whereas low protein concentrations are likely to yield lower FT4 values. In order to decrease nonspecific binding and neutralize the effect of nonesterified fatty acids on serum FT4, albumin is added in some assays. Albumin itself, however, binds T4 and when added in sufficient amounts, may disrupt the equilibrium. Nevertheless, the currently used FT4 immunoassays perform reasonably well in many circumstances, and most often accurately report both low FT4 levels in the setting of thyroid hormone deficiency and high FT4 levels in the setting of thyroid hormone excess $(26,27)$.

Sera of pregnant women are characterized by higher concentrations of TBG and nonesterified fatty acids and by lower concentrations of albumin relative to the sera of nonpregnant women. In part because of this, many current FT4 analog immunoassays fail dilutional assessment $(25,28)$. Because FT4 reference intervals in pregnancy vary widely between methods, interpretation of FT4 values requires method-specific as well as trimester-specific ranges $(10,11,27)$. Whereas it is customary for manufacturers to suggest that laboratories establish their own reference range for such a test, this suggestion is frequently impractical for FT4 assessment because it is especially difficult to recruit subjects with specific conditions such as pregnancy from which to independently establish method- and trimesterspecific reference ranges. Therefore, laboratories customarily adopt the pregnancy ranges provided by the test manufacturers. Typically, the characteristics of these reference pregnant cohorts are not disclosed, and differences in iodine intake and ethnicity may compromise the ability to generalize the manufacturer ranges across different populations. This problem adds to the complexity of accurate measurement of serum FT4 in the pregnant individual.

Current uncertainty around FT4 estimates in pregnancy has led some to question the wisdom of relying on any FT4 immunoassays during pregnancy $(29,30)$. In contrast, measurement of TT4 and the calculated FT4 index do show the expected inverse relationship with serum TSH (29). This finding suggests that TT4 measurements may be superior to immunoassay measurement of FT4 measurements in pregnant women. However, reference values should take the $50 \%$ increase in TBG witnessed during pregnancy into account by calculating the FT4 index using a serum thyroid hormone uptake test (such as the thyroid hormone binding ratio). Changes in total serum T4 concentration through pregnancy among euthyroid women have been previously reported (5). Changes are predictable, with an increase in TT4 concentration from weeks $7-16$ of gestation, ultimately reaching $\sim 50 \%$ above the prepregnancy level. This level is then sustained through pregnancy. Therefore, a clinically acceptable upper range determination can be calculated by shifting the nonpregnant limit 50\% higher. However, this limit can only be used after week 16 of pregnancy. If a T4 measurement is required before that time (i.e., weeks 7-16 of pregnancy), a calculation can be made for the upper reference range based on increasing the nonpregnant upper reference limit by $5 \%$ per week, beginning with week 7 . For example, at 11 weeks of gestation ( 4 weeks beyond week 7 ), the upper reference range for T4 is increased by $20 \%$ (4 weeks $\times 5 \% /$ week) (5).

As described above, free thyroid hormones can also be measured in the dialysate or ultrafiltrate using online solid phase extraction (LC/MS/MS). However, this approach is time con- suming, costly, and often impractical. Using direct equilibrium dialysis and LC/MS/MS, the 95\% FT4 reference intervals decrease gradually with advancing gestational age: from 1.08$1.82 \mathrm{ng} / \mathrm{dL}$ in week 14 to $0.86-1.53 \mathrm{ng} / \mathrm{dL}$ in week 20 (31). Serum FT4 by LC/MS/MS correlates very well with serum FT4 measured by classical equilibrium dialysis, but correlation with results from the FT4 immunoassay are less satisfactory (8). The use of isotope dilution-LC/MS/MS for measuring T4 in the dialysate from equilibrium dialysis of serum is helpful to obtain a gold-standard reference measurement procedure for serum FT4 (32). This assay technology, unfortunately, is currently not widely available due to high instrument and operating costs.

\section{- RECOMMENDATION 2}

The accuracy of serum FT4 measurement by the indirect analog immunoassays is influenced by pregnancy and also varies significantly by manufacturer. If measured in pregnant women, assay method-specific and trimesterspecific pregnancy reference ranges should be applied.

Strong recommendation, moderate-quality evidence.

\section{- RECOMMENDATION 3}

In lieu of measuring FT4, TT4 measurement (with a pregnancy-adjusted reference range) is a highly reliable means of estimating hormone concentration during the last part of pregnancy. Accurate estimation of the FT4 concentrations can also be done by calculating a FT4 index.

\section{Strong recommendation, moderate-quality evidence.}

\section{IODINE STATUS AND NUTRITION}

Because of increased thyroid hormone production, increased renal iodine excretion, and fetal iodine requirements, dietary iodine requirements are higher in pregnancy than they are for nonpregnant adults (33). Women with adequate iodine intake before and during pregnancy have adequate intrathyroidal iodine stores and have no difficulty adapting to the increased demand for thyroid hormone during gestation. In these women, total-body iodine levels remain stable throughout pregnancy (34). However, in areas of even mild to moderate iodine deficiency, total-body iodine stores, as reflected by urinary iodine values, decline gradually from the first to the third trimester of pregnancy (35). Iodine, required for infant nutrition, is secreted into breast milk. Therefore, lactating women also have increased dietary iodine requirements (36).

Spot urinary iodine values are used most frequently for determination of iodine status in populations. Because there is substantial diurnal and day-to-day variation in urinary iodine excretion, urinary iodine concentrations (UICs) cannot be used to identify particular individuals with iodine deficiency $(37,38)$. Therefore, iodine levels are a population rather than individual marker and outside unusual settings urinary iodide testing is not beneficial for individual use.

\section{- RECOMMENDATION 4}

Median UICs can be used to assess the iodine status of populations, but single spot or 24-hour UICs are not a valid marker for the iodine nutritional status of individual patients.

Strong recommendation, high-quality evidence. 
Question 4: What is the impact of severe iodine deficiency on the mother, fetus, and child?

Maternal dietary iodine deficiency results in impaired maternal and fetal thyroid hormone synthesis. Low thyroid hormone values stimulate increased pituitary TSH production, and the increased TSH stimulates thyroid growth, resulting in maternal and fetal goiter (39). In areas of severe iodine deficiency, thyroid nodules can be present in as many as $30 \%$ of pregnant women (40). Severe iodine deficiency in pregnant women has been associated with increased rates of pregnancy loss, stillbirth, and increased perinatal and infant mortality (41).

Normal levels of thyroid hormone are essential for neuronal migration, myelination, and other structural changes of the fetal brain. Because thyroid hormones are needed throughout pregnancy, iodine deficiency affects both maternal and fetal thyroid hormone production, and insufficient iodine intake can lead to detrimental effects. Specifically, maternal and fetal iodine deficiency in pregnancy have adverse effects on the cognitive function of offspring (42-45). Children whose mothers were severely iodine deficient during pregnancy may exhibit cretinism, characterized by profound intellectual impairment, deaf-mutism, and motor rigidity. Iodine deficiency is the leading cause of preventable intellectual deficits worldwide (42).

Universal salt iodization is the most cost-effective way of delivering iodine and improving maternal and infant heath (46).

\section{Question 5: What is the impact of mild to moderate iodine deficiency on the mother, fetus, and child?}

Groups of pregnant women whose median UICs are 50 $150 \mu \mathrm{g} / \mathrm{L}$ are defined as mildly to moderately iodine deficient. Women with mild to moderate iodine deficiency during pregnancy are at increased risk for the development of goiter (39) and thyroid disorders (47). Low maternal UIC in pregnancy has been associated with reduced placental weight and neonatal head circumference (48). However, in areas with adequate dietary iodine intake, variations in maternal UICs have a limited influence on physical developmental outcomes (49). Mild to moderate maternal iodine deficiency has also been associated with attention deficit and hyperactivity disorders in children (50) as well as impaired cognitive outcomes (51-53). In an iodine-deficient area iodized salt intake before pregnancy did improve maternal thyroid function; no difference in child neurodevelopment was noted (54), but improvement has been noted in other studies (55).

\section{Question 6: What is the iodine status of pregnant and breastfeeding women in the United States?}

Surveillance of urinary iodine values of the U.S. population has been carried out at intervals since 1971. Following a precipitous drop in urinary iodine values between 1971 and 1994, U.S. dietary iodine intake has stabilized (56-61). The U.S. population overall remains iodine sufficient. However, U.S. women of reproductive age are the most likely group to have low urinary iodine values. According to the World Health Organization (WHO) guidelines, median urinary iodine values for pregnant women between 149 and $249 \mu \mathrm{g} / \mathrm{L}$ are consistent with optimal iodine intake (42). In the 20052010 National Health and Nutrition Examination Survey (NHANES) surveys, the median UIC for U.S. pregnant women was $129 \mu \mathrm{g} / \mathrm{L}$, consistent with mild iodine deficiency (62). Current data regarding iodine sufficiency among lactating U.S. women are very limited. It is possible that a subset of pregnant and lactating U.S. women may have mildly to moderately inadequate dietary iodine intake resulting in insufficient amounts of iodine in the breast milk to meet infants' dietary requirements, but studies have been small and inconsistent (63-65).

\section{Question 7: What is the iodine status of pregnant and breastfeeding women worldwide?}

Since 1990, the number of households worldwide using iodized salt has risen from less than $20 \%$ to more than $70 \%$ (66). Despite these advances, however, 30 countries remain iodine deficient, and iodine deficiency remains the leading cause of preventable intellectual deficits worldwide (44). Recent reports highlight the value of iodized salt in correcting iodine deficiency states in India $(67,68)$, although remaining iodine deficiency was noted in one report (69). Only $6 \%$ of pregnant women had low UICs in rural Bangladesh (70), whereas $80 \%$ had low urine iodine concentrations in Pakistan (71). In Shanghai, China, iodine deficiency was noted in a subset of pregnant women (72), whereas in Shenyang city slight iodine excess was noted, with a consequent increase in subclinical hypothyroidism (73). Iodine status in Korea is more than adequate (median UIC $427 \mu \mathrm{g} / \mathrm{L}$ ) (74). Iodine status in Japan is also more than adequate (median UIC $328 \mu \mathrm{g} / \mathrm{L})(9)$. On the African continent, iodine status in pregnancy was inadequate in Niger (75) and was also poor in Ethiopia (76). Iodine nutrition in Iran was sufficient (77). In Brazil, median UIC was $138 \mu \mathrm{g} / \mathrm{L}$, possibly due to reduced concentration of iodine in salt (78). In Europe many countries, including Belgium, the Czech Republic, Denmark, France, Latvia, Norway, Spain, and the United Kingdom, have recorded significant iodine deficiency in their pregnant populations $(47,79-85)$.

\section{Question 8: Does iodine supplementation in pregnancy and lactation improve outcomes in severe iodine deficiency?}

In areas of severe iodine deficiency, iodine supplementation of mothers prior to conception or in early pregnancy results in children with improved cognitive performance relative to children of mothers given a placebo (86-88). The prevalence of cretinism and other severe neurological abnormalities is significantly reduced (89). Maternal iodine supplementation in severely iodine-deficient areas also decreases rates of stillbirth and neonatal and infant mortality $(90,91)$. Oral administration of iodized oil can increase birth weight in addition to correcting iodine deficiency (92).

A recent randomized clinical trial demonstrated that in moderately to severely iodine-deficient areas without universal salt iodization, lactating women who receive one dose of $400 \mathrm{mg}$ oral iodized oil after delivery can provide adequate iodine to their infants through breast milk for at least 6 months. Direct infant iodine supplementation was less effective at improving infant iodine status (93). 


\section{Question 9: Does iodine supplementation in pregnancy and lactation improve outcomes in mildly to moderately iodine-deficient women?}

Eight controlled trials of iodine supplementation in mildly to moderately iodine-deficient pregnant European women have been published (94-101), although doses and timing of iodine supplementation varied and only two trials examined effects on offspring development. Iodine supplementation of moderately deficient pregnant women appears to consistently decrease maternal and neonatal thyroid volumes and thyroglobulin ( $\mathrm{Tg}$ ) levels. Effects on maternal thyroid function have been mixed, with significant maternal TSH decreases with supplementation described in four $(88,90,91,95)$ of the eight published trials, and increases in maternal T4 or FT4 noted in just two $(90,95)$. A reduction in cord TSH also indicates improvement in gestational iodine status (102).

In two nonrandomized studies, neurodevelopmental outcomes were improved in children from mildly to moderately iodine-deficient areas whose mothers received iodine supplementation early in pregnancy $(87,95)$. Another study failed to show neuropsychological improvement in 16-month-old children of mothers who received supplementation (103). The timing of supplementation is likely to be critical because the beneficial effects of iodine on offspring development appeared to be lost if supplementation is started after 10-20 weeks gestation. If iodine supplementation is started before pregnancy in iodine-deficient women better maternal thyroid function can be observed but, depending on dose and the timing of initiation, supplementation may not fully correct iodine deficiency in an already iodine-deficient population (104). A meta-analysis concluded that iodine supplementation improves some maternal thyroid indices and may benefit aspects of cognitive function in school age children, even in marginally iodine-deficient areas (105). Another review highlighted the lack of high-quality evidence in relation to these outcomes and suggested that randomized controlled trial (RCTs) may not be feasible where iodine supplementation is common (106).

Recently, there has been controversy regarding whether it is ethical to perform randomized clinical trials of iodine supplementation in pregnancy in regions that are mildly to moderately iodine deficient (107-109). Caution in accepting the necessity of supplementation has been expressed, especially in areas where iodized salt is already in use (110).

No trials to date have specifically examined the effects of iodine supplementation in lactation in mildly to moderately iodine-deficient regions.

\section{Question 10: What is the recommended daily iodine intake in women planning pregnancy, women who are pregnant, and women who are breastfeeding?}

Iodine is an essential nutrient required for thyroid hormone production and is primarily derived from the diet and from vitamin/mineral preparations. The U.S. Institute of Medicine recommended dietary allowances to be used as goals for individual total daily iodine intake (dietary and supplement) are $150 \mu \mathrm{g} / \mathrm{d}$ for women planning a pregnancy, $220 \mu \mathrm{g} / \mathrm{d}$ for pregnant women, and $290 \mu \mathrm{g} / \mathrm{d}$ for women who are breastfeeding (111). The WHO recommends $250 \mu \mathrm{g} / \mathrm{d}$ for pregnant and lactating women (39). This level is supported by a study of more than 7000 pregnant Chinese women in whom it was found that subclinical hypothyroidism and hypothyroxinemia were least common at the urinary iodine range of 150$249 \mu \mathrm{g} / \mathrm{L}$, but risk for both these abnormalities rose when the UIC was lower or higher than this range (73).

Dietary iodine sources vary regionally. Sources of iodine in the United States diet have been difficult to identify, in part because there are a wide variety of potential sources and food iodine content is not listed on packaging. Iodized salt remains the mainstay of iodine deficiency disorder elimination efforts worldwide. However, salt iodization has never been mandated in the United States and only approximately $50 \%$ of salt sold for household use in the United States is iodized (112). In the United States, dairy foods are another important source of dietary iodine because of iodine in cattle feed and the use of iodophor disinfectants by the dairy industry (113-115). Commercially baked breads have been another major source of iodine in the United States because of the use of iodate bread conditioners (115). However, the use of iodate bread conditioners has decreased over the past several decades. Other sources of iodine in the United States diet are seafood, eggs, meat, and poultry (116). Foods of marine origin generally have high concentrations of iodine because marine animals concentrate iodine from seawater (96-98), although the amount of iodine in different fish and shellfish species is quite variable.

In the United States, the dietary iodine intake of individuals cannot be reliably ascertained either by patient history or by any laboratory measure. Because of concerns that a subset of pregnant U.S. women may be mildly to moderately iodine deficient and an inability to identify individual women who may be at risk, the ATA has previously recommended $150 \mu \mathrm{g}$ daily iodine supplementation for all North American women who are pregnant or breastfeeding (117). More recently the Endocrine Society, Teratology Society, and American Academy of Pediatrics have also advocated iodine supplementation for pregnant and lactating U.S. women $(15,118,119)$. The goal is supplementation of dietary iodine intake, rather than its replacement. Special attention may need to be paid to those with dietary restrictions (e.g., lactose intolerant, gluten intolerant, low-carbohydrate, or vegan) because those individuals may have additional needs for supplementation (120).

Unfortunately, recommendations regarding iodine supplementation in the United States have not been widely adopted. In the NHANES 2001-2006 dataset, only 20\% of pregnant women and $15 \%$ of lactating women reported ingesting iodine-containing supplements (121). When assessed in 2009 , of the 223 types of prenatal multivitamins available in the United States, only 51\% contained any iodine (122). However, this may recently have changed; in 2015 the Council for Responsible Nutrition, the U.S. supplement industry trade group, recommended that their members include $150 \mu \mathrm{g}$ iodine in all prenatal multivitamin preparations. Iodine in U.S. prenatal multivitamins is typically derived either from potassium iodide or from kelp. The iodine content in prenatal multivitamin brands containing kelp may be inconsistent because of variability in kelp iodine content (123). Women consuming levothyroxine (LT4) regularly do not require supplemental iodine because the substrate is no longer needed for hormone formation.

The task force recommendations for iodine supplementation in the setting of lactation and breastfeeding are provided in Section XI. 


\section{- RECOMMENDATION 5}

All pregnant women should ingest approximately $250 \mu \mathrm{g}$ iodine daily. To achieve a total of $250 \mu \mathrm{g}$ iodine ingestion daily, strategies may need to be varied based on country of origin.

\section{Strong recommendation, high-quality evidence.}

\section{- RECOMMENDATION 6}

In most regions, including the United States, women who are planning pregnancy or currently pregnant, should supplement their diet with a daily oral supplement that contains $150 \mu \mathrm{g}$ of iodine in the form of potassium iodide. This is optimally started 3 months in advance of planned pregnancy.

Strong recommendation, moderate-quality evidence.

\section{- RECOMMENDATION 7}

In low-resource countries and regions where neither salt iodization nor daily iodine supplements are feasible, a single annual dose of $\sim 400 \mathrm{mg}$ of iodized oil for pregnant women and women of childbearing age can be used as a temporary measure to protect vulnerable populations. This should not be employed as a long-term strategy or in regions where other options are available.

Weak recommendation, moderate-quality evidence.

\section{- RECOMMENDATION 8}

There is no need to initiate iodine supplementation in pregnant women who are being treated for hyperthyroidism or who are taking LT4.

Weak recommendation, low-quality evidence.

\section{Question 11: What is the safe upper limit for iodine consumption in pregnant and breastfeeding women?}

Most people are tolerant of chronic excess dietary iodine intake due to a homeostatic mechanism known as the WolffChaikoff effect $(124,125)$. In response to a large iodine load, there is a transient inhibition of thyroid hormone synthesis. Following several days of continued exposure to high iodine levels, escape from the acute Wolff-Chaikoff effect is mediated by a decrease in the active transport of iodine into the thyroid gland, and thyroid hormone production resumes at normal levels (126).

Some individuals do not appropriately escape from the acute Wolff-Chaikoff effect, making them susceptible to hypothyroidism in the setting of high iodine intake. The fetus may be particularly susceptible, since the ability to escape from the acute Wolff-Chaikoff effect does not fully mature until about week 36 of gestation $(127,128)$.

Tolerable upper intake levels for iodine have been established to determine the highest level of daily nutrient intake that is likely to be tolerated biologically and to pose no risk of adverse health effects for almost all individuals in the general population. The upper intake levels are based on total intake of a nutrient from food, water, and supplements and apply to chronic daily use. The U.S. Institute of Medicine has defined the tolerable upper limit for daily iodine intake as $1100 \mu \mathrm{g} / \mathrm{d}$ in all adults, including pregnant women (96) and the WHO has stated that daily iodine intake
$>500 \mu \mathrm{g}$ may be excessive in pregnancy. Recent population data support the WHO threshold (73). The exception may be communities that have historically and consistently consumed greater than $500 \mu \mathrm{g}$ daily without experiencing adverse effects (e.g., Japan).

Medications may be a source of excessive iodine intake for some individuals. Amiodarone, an antiarrhythmic agent (129), contains $75 \mathrm{mg}$ iodine per $200 \mathrm{mg}$ tablet. Iodinated intravenous radiographic contrast agents contain up to $380 \mathrm{mg} / \mathrm{mL}$. Some topical antiseptics contain iodine, although systemic absorption is generally not clinically significant in adults except in patients with severe burns (130). Iodine-containing anti-asthmatic medications and expectorants are occasionally used. In addition, some dietary supplements such as kelp and some iodine preparations may contain very large amounts of iodine (several thousand times higher than the daily upper limit) and should not be taken. Ingestion of iodine and kelp supplements containing in excess of $500 \mu \mathrm{g} / \mathrm{d}$ is not recommended in pregnancy or lactation (131).

Concern exists that some populations may be exposed to excess iodine, possibly resulting in a high prevalence of thyroid dysfunction $(132,133)$, an increased rate of hyperthyrotrophinemia (134), and an increased rate of hyperthyroid newborns (135). In addition, iodine-induced hypothyroidism has been reported in infants exposed to excess iodine from radiocontrast agents (136). It should be recognized that even low-dose iodine supplementation may trigger thyroid autoimmunity in a small proportion of women (73). The exception may be communities that have historically and consistently consumed greater then $500 \mu \mathrm{g}$ daily without experiencing adverse effects. In one study, approximately one third of Japanese pregnant women demonstrated higher UIC beyond $500 \mu \mathrm{g} / \mathrm{Cr}$, but there were no problems in pregnancy progress and fetal development (9).

\section{- RECOMMENDATION 9}

Excessive doses of iodine exposure during pregnancy should be avoided, except in preparation for the surgical treatment of GD. Clinicians should carefully weigh the risks and benefits when ordering medications or diagnostic tests that will result in high iodine exposure.

\section{Strong recommendation, moderate-quality evidence.}

\section{- RECOMMENDATION 10}

Sustained iodine intake from diet and dietary supplements exceeding $500 \mu \mathrm{g}$ daily should be avoided during pregnancy due to concerns about the potential for fetal thyroid dysfunction.

Strong recommendation, moderate-quality evidence.

\section{THYROID AUTO-ANTIBODIES AND PREGNANCY COMPLICATIONS}

\section{Question 12: What is the prevalence of thyroid autoantibodies in pregnant women?}

Anti-TPO or anti-Tg thyroid autoantibodies are present in $2 \%$ to $17 \%$ of unselected pregnant women $(47,137-144)$. The prevalence of antibodies varies with ethnicity. In U.S. populations, thyroid antibodies are most frequent in Caucasian and Asian women and least frequent in African Americans 
$(139,145)$. Dietary iodine intake may also be associated with anti-thyroid $\mathrm{Ab}$ positivity during pregnancy. Shi and colleagues (73) recently demonstrated a U-shaped relationship between UICs and antibody positivity among pregnant women.

A recent study from Belgium in women seeking fertility treatment showed that both TPOAb and $\mathrm{TgAb}$ were present in $8 \%$ of women, while $5 \%$ demonstrated isolated $\mathrm{Tg}$ antibodies and $4 \%$ demonstrated isolated TPOAb concentrations (146). Those women with isolated $\mathrm{TgAb}$ positivity had a significantly higher serum TSH than women without thyroid autoimmunity. While the task force acknowledges that testing for thyroid autoimmunity using only TPOAb would likely miss a small proportion of women with isolated Tg antibodies, we note that the vast majority of studies investigating thyroid autoimmunity and clinical outcomes used only TPOAb measurements. For this reason, the task force recommends assessment of TPOAb when testing for the presence of thyroid autoimmunity.

\section{Question 13: What is the natural history of antithyroid antibodies in pregnant women?}

\section{Question 14: How should euthyroid women who are thyroid antibody (Ab)-positive be monitored during pregnancy?}

In women with thyroid autoimmunity, hypothyroidism may occur because of the stress of pregnancy because the ability of the thyroid to augment hormone production is compromised. In 1994, Glinoer et al. (147) performed a prospective study of 87 euthyroid (TSH $\leq 4 \mathrm{mU} / \mathrm{L})$ women who were positive for TPOAb, TgAb, or both. Twenty percent of women in the study developed a serum TSH $>4 \mathrm{mU} / \mathrm{L}$ during gestation despite normal prepregnancy TSH values. Antithyroid Ab titers were highest in the first trimester, although they decreased by about $60 \%$ over the course of gestation. Twelve years later, in a prospective study, Negro et al. (28) demonstrated similar results. The authors found that in TPOAb-positive euthyroid women, TSH levels increased as gestation progressed, from a mean of $1.7 \mathrm{mU} / \mathrm{L}$ (12th week) to $3.5 \mathrm{mU} / \mathrm{L}$ (term), with $19 \%$ of women having a supranormal TSH value at delivery. Because the risk of TSH elevation is increased in this population, increased surveillance of euthyroid thyroid $\mathrm{Ab}$-positive women should occur.

TPO antibodies are able to cross the placenta. At the time of delivery, cord blood TPOAb levels strongly correlate with third-trimester maternal TPOAb concentrations (148). However, maternal passage of either TPOAb or TgAb is not associated with fetal thyroid dysfunction.

\section{- RECOMMENDATION 11}

Euthyroid pregnant women who are TPOAb or TgAb positive should have measurement of serum TSH concentration performed at time of pregnancy confirmation and every 4 weeks through midpregnancy.

\section{Strong recommendation, high-quality evidence.}

\section{Question 15: Should euthyroid women with thyroid autoimmunity be treated with selenium?}

Some studies evaluating nonpregnant women have shown that selenium can diminish TPOAb concentrations (149-152).
However, this reduction has not been observed in all studies (153). Negro et al. (154) noted that euthyroid TPOAbpositive pregnant women randomized to treatment with $200 \mu \mathrm{g} / \mathrm{d}$ selenium not only had a significant decrease in the frequency of postpartum thyroid dysfunction $(p<0.01)$ but also had lower TPOAb concentrations during pregnancy compared to those in the untreated group. Importantly, this trial did not measure urinary iodine, a potential confounder because iodine status may influence the thyroidal effects of selenium. However, in another recent randomized clinical trial (155) performed in mildly iodine-deficient British pregnant women, treatment with $60 \mu \mathrm{g}$ of selenium daily did not affect TPO concentrations or TPOAb positivity. Thus, conflicting data regarding selenium supplementation make any generalized recommendation unreliable, especially to regions with different intakes of iodine, selenium, or both. In addition, patients treated with selenium could be at higher risk for developing type 2 diabetes mellitus (156). For these reasons, the risk-to-benefit comparison does not presently support routine selenium supplementation of TPOAb-positive women during pregnancy.

\section{- RECOMMENDATION 12}

Selenium supplementation is not recommended for the treatment of TPOAb-positive women during pregnancy.

Weak recommendation, moderate-quality evidence.

\section{Question 16: Is there an association between thyroid antibodies and sporadic spontaneous pregnancy loss in euthyroid women?}

Spontaneous pregnancy loss (miscarriage), occurs in $17 \%$ $31 \%$ of all gestations $(157,158)$. A spontaneous pregnancy loss is usually defined as one occurring at less than 20 weeks of gestation. The individual risk varies according to clinical factors including maternal age, family history, environmental exposures, and medical comorbidities (159). Pregnancy losses are a significant emotional burden to patients and may also result in bleeding, infection, pain, and need for surgical intervention.

Endocrine disorders have been previously recognized as risk factors for spontaneous pregnancy loss. Patients with poorly controlled diabetes mellitus may have up to a $50 \%$ risk of loss (160). Thyroid dysfunction has similarly been associated with increased pregnancy loss (161). Stagnaro-Green and colleagues (162) first demonstrated an association between pregnancy loss and thyroid antibodies in a prospective observational study. Patients who were positive for thyroid antibodies (TPOAb, TgAb, or both) demonstrated a 2-fold increase in the risk for pregnancy loss (17\% vs. $8.4 \%$, $p=0.01)$. Since that time, numerous other studies have examined the association between maternal antithyroid $\mathrm{Ab}$ status and pregnancy loss risk, showing similar findings. In a recent meta-analysis of eight case-control studies, the pooled OR for pregnancy loss in women with thyroid autoimmunity versus women without antithyroid antibodies was 2.55 [95\% CI 1.42-4.57]. Meta-analysis of 14 cohort studies showed a similar increased OR of 2.31 [95\% CI 1.90-2.82] (163). However, Ab-positive women were noted to be slightly older and to have slightly higher serum TSH values. In support of these data, a separate meta-analysis similarly found an increase in loss rate among thyroid $\mathrm{Ab}$-positive women (OR 
3.90 for cohort studies [95\% CI 2.48-6.12]; OR 1.80 for case control studies [95\% CI 1.25-2.60]) (164).

Although a clear association has been demonstrated between thyroid antibodies and spontaneous pregnancy loss, it does not prove causality and the underlying mechanisms for such an association remain unclear. Three research groups have demonstrated one possible mechanism through increased fetal resorption in active immunization murine models (165-167). Several other mechanistic hypotheses have been proposed, including Ab-mediated mild thyroid hypofunction, cross-reactivity of antithyroid antibodies with hCG receptors on the zona pellucida, the presence of concurrent non-organ-specific autoimmunity, and increased levels of endometrial cytokines in women with thyroid autoimmunity (168).

\section{Question 17: Is there an association between thyroid antibodies and recurrent spontaneous pregnancy loss in euthyroid women?}

Recurrent pregnancy loss is defined as either two consecutive spontaneous losses or three or more spontaneous losses, and it may occur in up to $1 \%$ of all women (169). Several causes have been reported, including parental chromosomal anomalies, immunologic derangements, uterine pathology, and endocrine dysfunction (170).

In a case-control study, Iravani and colleagues (171) reported that patients with primary recurrent pregnancy losses (three or more) had a higher incidence of $\mathrm{TgAb}$ and/or TPOAb positivity (OR 2.24 [95\% CI 1.5-3.3]). Kutteh et al. (172) reported similar findings, with an increased rate of positivity for $\mathrm{TgAb}, \mathrm{TPOAb}$, or both in 700 women with recurrent pregnancy loss compared to 200 healthy controls $(22.5 \%$ vs. $14.5 \%, p=0.01)$. On the other hand, in a prospective observational study, Esplin and colleagues (173) demonstrated no difference in positivity for $\mathrm{TgAb}$, TPOAb, or both between patients with recurrent pregnancy loss and healthy controls. Pratt and colleagues (174) reported a higher rate of subsequent pregnancy loss in patients with recurrent losses and thyroid Ab positivity. In a larger study with a similar population, Rushworth and colleagues (175) reported no significant difference in live birth rates between women with recurrent losses who were $\mathrm{TgAb}$ or thyroid microsomal $\mathrm{Ab}$ positive and those who were not. In a casecontrol study, Lata et al. (176) found that the prevalence of TPO antibodies was higher in women with recurrent pregnancy loss than in healthy pregnant controls without a history of recurrent loss $(31 \%$ vs. $18 \%$. $p=0.031)$. A metaanalysis of eight studies that included $460 \mathrm{Ab}$-positive patients and 1923 controls noted a significant association between thyroid $\mathrm{Ab}$ positivity and recurrent pregnancy loss (OR 2.3 [95\% CI 1.5-3.5]) (177).

Thus, the data for an association between thyroid antibodies and recurrent pregnancy loss are less robust than for sporadic loss. This finding may be because recurrent pregnancy loss has many potential causes, and endocrine dysfunction may only account for $15 \%-20 \%$ of all such cases (170). Many of the studies described above did not control for other potential causes of recurrent losses. One intriguing study reported an apparent interaction of antiphospholipid antibodies and antithyroid antibodies in the risk for recurrent pregnancy loss (178). In support of this, Kim and colleagues (179) reported that women with recurrent pregnancy loss who were antithyroid $\mathrm{Ab}$ positive also demonstrated higher levels of anticardiolipin Ab and other nonorgan-specific antibodies.

\section{Question 18: Does treatment with LT4 or intravenous immunoglobulin therapy decrease the risk for pregnancy loss in euthyroid women with thyroid autoimmunity?}

Negro and colleagues (28) reported a prospective, randomized interventional trial of LT4 in euthyroid patients who were TPOAb positive. The researchers reported a significantly decreased rate of pregnancy loss in the LT4-treated group (3.5\% vs. $13.8 \%, p<0.05)$. A limitation of the study is that the mean estimated gestational age at starting LT4 was 10 weeks, and all but one of the eight losses in the untreated group had occurred before 11 weeks. This finding raises uncertainty as to the impact of the intervention upon the endpoint.

However, in a separate nonrandomized, retrospective study, Lepoutre et al. (180) analyzed data from 65 TPOAbpositive pregnant women with serum TSH values of 1$3.5 \mathrm{mU} / \mathrm{L}$ at the first antenatal visit. Thirty-four of these women were treated with $50 \mu \mathrm{g} \mathrm{LT} 4$ daily starting at a mean 10 weeks gestation, while the others were not treated. None of the LT4-treated women miscarried, but 5 of 31 untreated women $(16 \%)$ experienced pregnancy loss. Although limited in nature, these data support the findings of Negro et al. (28) and begin to suggest a potential benefit to this treatment approach. The underlying mechanism is, however, uncertain.

Three small nonrandomized case series have been published on the use of intravenous immunoglobulin (IVIG) therapy for the prevention of recurrent pregnancy loss in women with antithyroid antibodies (181-183). The live birth rates ranged from $80 \%$ to $95 \%$, and the one study with a control group (consisting of women who refused IVIG therapy) reported a highly significant improvement in live births in the IVIG-treated cohort ( $95 \%$ vs. $0 \% p=0.001)(182)$. Comparison of a LT4 intervention to an IVIG intervention in one study demonstrated a higher rate of term delivery in the LT4-treated group (183). However, all three studies had serious design flaws including small sample sizes, heterogeneous patient populations, lack of or limited randomization, and differences in the timing of treatment initiation. Nonetheless, intervention trials with LT4 (and less so IVIG) in euthyroid TPOAb-positive women with recurrent abortion appear to show a possible decrease in miscarriage rates. Further randomized trials are needed to better understand the effectiveness of both LT4 and IVIG intervention. At present, however, the cost, complexity, and side effect profile associated with IVIG infusion must be noted and make its use undesirable given the questionable benefit. In contrast, LT4 administration in low dosage $(25-50 \mu \mathrm{g} / \mathrm{d})$ is safe. Therefore, its use among patients with recurrent pregnancy loss may be reasonably considered in the setting of early gestation, especially when no other known cause of prior pregnancy loss has been identified.

The task force makes note that two randomized clinical trials are currently ongoing. The Thyroid AntiBodies and LEvoThyroxine study (TABLET) trial in the United Kingdom is randomizing euthyroid TPOAb-positive women with a 
history of infertility or recurrent losses to either LT4 or placebo to assess effects on live birth rates. Separately, the T4Lifetrial in the Netherlands is examining the effects of LT4 treatment in euthyroid pregnant women with a history of recurrent loss. The primary outcome of this trial is the live birth rate.

\section{- RECOMMENDATION 13}

Intravenous immunoglobulin treatment of euthyroid women with a history of recurrent pregnancy loss is not recommended.

Weak recommendation, low-quality evidence.

\section{- RECOMMENDATION 14}

Insufficient evidence exists to conclusively determine whether LT4 therapy decreases pregnancy loss risk in TPOAb-positive euthyroid women who are newly pregnant. However, administration of LT4 to TPOAb-positive euthyroid pregnant women with a prior history of loss may be considered given its potential benefits in comparison with its minimal risk. In such cases, $25-50 \mu \mathrm{g}$ of LT4 is a typical starting dose.

\section{Weak recommendation, low-quality evidence.}

\section{Question 19: Is there an association between thyroid autoantibody positivity and premature delivery?}

Preterm deliveries are defined as those occurring before 37 weeks gestation. In the United States, $11.4 \%$ of all births are preterm (184). Preterm birth remains one of the most prevalent and morbid perinatal complications. It is the leading cause of neonatal death and is associated with increased risks for acute respiratory, gastrointestinal, immunologic, central nervous system (CNS), hearing, and vision problems, as well as longer-term motor, cognitive, visual, hearing, behavioral, and growth impairment (185). A decade ago, the annual cost of preterm delivery to the U.S. health care system was estimated at $\$ 26$ billion (185). Preterm birth has remained difficult to predict, prevent, and treat primarily because there are multiple potential causes and pathways that end in premature labor (186). Examples include infection, trauma, cervical insufficiency, premature rupture of membranes, and maternal medical conditions.

The relationship between thyroid autoantibodies and preterm delivery has been investigated with mixed results. Glinoer et al. (187) reported in a prospective cohort that euthyroid women positive for either TPOAb or TgAb had a significantly increased incidence of preterm birth (16\% vs. $8 \%, p<0.005)$. Ghafoor et al. (188) evaluated 1500 euthyroid women and found an increase in preterm delivery in TPOAbpositive women compared to women who were TPOAb negative $(26.8 \%$ vs. $8.0 \%, p<0.01)$. In contrast, Iijima et al. (189) did not find an increased risk for preterm birth in women positive for seven different autoantibodies and thyroid antibodies. This study had an unusually low rate of preterm birth in both study and control groups (3\% vs. $3.1 \%)$. Haddow et al. (190) reported a significant increase in preterm premature rupture of the membranes, but not in preterm delivery, among women who were TPOAb and/or TgAb positive in the first trimester.
Importantly, three recent large prospective cohort studies failed to find significant associations between antithyroid antibody positivity and risk for premature delivery. However, in each of the studies, prematurity rates were nonsignificantly higher in TPOAb-positive women than in women who were antibody negative $(19,190,191)$. By contrast, Karakosta et al. (192) found that euthyroid women (TSH $<2.5 \mathrm{mIU} / \mathrm{L})$ with positivity for TPOAb, TgAb, or both in early pregnancy were at increased risk for spontaneous preterm delivery (RR 1.7 [95\% CI 1.1-2.8]). In a small cohort of 395 pregnant women, Kumru and colleagues (193) noted that spontaneous preterm delivery ( $<37$ weeks) was more frequent in TPOAb-positive euthyroid (TSH <2.6 mU/L) women (OR 2.5 [95\% CI 1.065.89]). Negro and colleagues found that untreated TPOAbpositive women had a substantially higher risk for preterm delivery than women without TPOAb (RR 12.18 [95\% CI 7.93-18.7]) (28). A 2011 meta-analysis of seven studies, including about 23,000 participants, concluded that there was an association between thyroid autoimmunity and preterm delivery (OR $=1.6$ [95\% CI 1.44-1.94]) (194). In support, a meta-analysis of five cohort studies including 12,566 women similarly concluded that a positive association existed between the presence of thyroid $\mathrm{Ab}$ and preterm birth (OR 2.907 [95\% CI 1.17-3.68]) (164). Finally, a third metaanalysis of 11 prospective cohorts including 35,467 participants determined that the relative risk for delivery at less than 37 weeks for women with positivity for TgAb, TPOAb, or both was 1.41 [95\% CI 1.08-1.84] (195). Interestingly, in subgroup analyses, TPOAb but not TgAb positivity was associated with prematurity. Together, these data suggest that thyroid autoantibody positivity is associated with increased risk for preterm delivery.

\section{Question 20: Does LT4 treatment of euthyroid women who are thyroid autoantibody positive reduce risk for premature delivery?}

In contrast to association studies, interventional studies of LT4 therapy for the prevention of preterm delivery are sparse. Negro et al. (28) reported an increased risk of preterm delivery among euthyroid TPOAb-positive women compared to euthyroid TPOAb-negative women in the only prospective interventional trial to date $(22.4 \%$ vs. $8.2 \%, p<0.01)$. The TPOAb-positive subjects were randomized to either treatment with LT4 or no treatment, with the LT4 dose based on TSH level. The treated group had a significantly lower rate of preterm delivery than did the untreated group (7\% vs. $22.4 \%$, $p<0.05)$. This finding has not been replicated. The ongoing TABLET study in the United Kingdom, a randomized clinical trial examining effects of LT4 treatment of euthyroid women with a history of infertility or recurrent pregnancy loss, will examine preterm birth as a secondary outcome. Therefore, at present, there are insufficient data from which to draw any conclusion regarding the utility of LT4 administration for the purpose of reducing preterm delivery.

\section{- RECOMMENDATION 15}

Insufficient evidence exists to recommend for or against treating euthyroid pregnant women who are thyroid autoantibody positive with LT4 to prevent preterm delivery.

No recommendation, insufficient evidence. 


\section{Question 21: Is thyroid autoimmunity in euthyroid pregnant women associated with adverse obstetric or child outcomes other than pregnancy loss and premature birth?}

Antithyroid antibodies have been associated with perinatal death in some $(191)$ but not all $(140,196)$ studies. In two pregnancy cohorts an increased risk for placental abruption was observed in women with thyroid autoimmunity and without clinical hypothyroidism $(140,190)$. Maternal thyroid autoimmunity has also been linked to postpartum depression $(197,198)$ and to neonatal respiratory distress syndrome (196).

Several studies have examined associations between maternal thyroid autoimmunity and child development. In a nested case-control study, Li et al. (199) reported lower motor and intellectual development at age 25-30 months in the offspring of euthyroid women who were TPOAb positive compared to children of TPOAb-negative controls. Williams et al. (200) followed 97 full-term mothers and their children, assessing child neurocognition at age 5.5 years. Lower perceptual performance and motor scores were described in children of TgAb-positive mothers, and lower perceptual performance scores noted in children with TgAb-positive cord blood. However, no neurodevelopmental outcomes were associated with maternal or infant TPOAb status. Wasserman and colleagues (141) described higher rates of sensorineural hearing loss $(22.7 \%$ vs. $4.3 \%, p=0.004)$ at age 8 in children whose mothers were TPOAb positive in pregnancy compared to children of TPOAb-negative women. Although maternal thyroid function was not assessed in this study, the association remained significant after controlling for a known history of maternal hypothyroidism. In the same cohort, cognitive assessments were performed in children at ages 4 and 7 years (201). Maternal TPOAb positivity was associated with lower child intelligence quotient (IQ) at age 4 , but effects were attenuated by age 7 . The authors speculated that the lower IQ scores at age 4 might be mediated by sensorineural hearing loss. Ghassabian and colleagues (202) assessed a cohort of 3139 mother-child pairs in which child cognitive function was assessed at age 2.5 years and behavior assessed at age 3. Maternal TPOAb status did not predict child neurocognition, but TPOAb positivity was associated with externalizing problems in children (OR 1.64 [95\% CI 1.17-2.29]), especially attention deficit/hyperactivity problems (OR 1.77 [95\% CI 1.15-2.72]). This result remained significant after adjustment for maternal TSH. Finally, in a nested case-control study, Brown et al. (203) found that women with children on the autism spectrum were more likely to have had positive TPOAb during pregnancy than mothers of controls.

\section{THE IMPACT OF THYROID ILLNESS UPON INFERTILITY AND ASSISTED REPRODUCTION}

Infertility is defined as the failure to achieve a clinical pregnancy after 12 or more months of regular unprotected sexual intercourse (204). Infertility affects $7.4 \%$ of U.S. women aged 15-44 years (205). Infertility is due to female factors in about $35 \%$ of cases, due to male factors in $30 \%$ of cases, and due to both female and male factors in $20 \%$ of cases. In approximately $15 \%$ of cases the cause of infertility is unknown (206).

\section{Question 22: Is overt thyroid dysfunction associated with infertility in women?}

Irregular menses may occur in women with overt hyperthyroidism. Krassas and colleagues (207) reported that menstrual irregularities were present in $22 \%$ of hyperthyroid patients as compared to $8 \%$ in age- and weight-matched euthyroid controls. However, in a cross-sectional study, the prevalence of hyperthyroidism (both subclinical and overt) was similar in infertile women compared to fertile controls (208).

The risk of infertility in women with overt hypothyroidism is less well studied. In a study of 171 hypothyroid women with TSH concentrations $>15 \mathrm{mU} / \mathrm{L}, 68 \%$ reported having irregular menses, far higher than the $12 \%$ rate of menstrual irregularities reported by euthyroid controls (209). In one cross-sectional study among 129 infertile women, 5\% had serum TSH levels $>4.5 \mathrm{mU} / \mathrm{L}$ (210). In a separate cross-sectional study among women age 18-50 years, the prevalence of infertility was $52.3 \%$ in women with GD and $47 \%$ among women with Hashimoto's thyroiditis; however, thyroid function was not reported in either group (211). Thus, despite imperfect data, the majority of evidence appears to support an association between overt thyroid dysfunction and an increased risk of infertility. Thyroid dysfunction is also reversible, and treatment is generally safe and may exert a positive effect on fertility. Therefore, it is reasonable to treat overt thyroid dysfunction in infertile women, with the goal of normalizing thyroid function.

\section{Question 23: Is subclinical hypothyroidism associated with infertility in women?}

Different definitions of subclinical hypothyroidism have been used in different studies examining this question, and results have been inconsistent. In a cross-sectional study of 704 women with infertility, the prevalence of TSH elevations was $2.3 \%$, similar to background rates in the general population (212). In a prospective study, Poppe and colleagues (208) did not find increased rates of subclinical hypothyroidism among infertile women but did report slightly higher median serum TSH levels (1.3 vs. $1.1 \mathrm{mU} / \mathrm{L}$ ) in the infertile women compared to controls. However, in a retrospective study, higher rates of subclinical hypothyroidism (13.9\% vs. $3.9 \%)$ were reported in infertile women as compared to fertile controls (213). Among women with baseline TSH values $2.5-5 \mathrm{mU} / \mathrm{L}$, thyrotropin releasing hormone (TRH) stimulation testing caused TSH increases to $>30 \mathrm{mU} / \mathrm{L}$ in $46 \%$ of women with female-factor infertility versus $7 \%$ of women whose infertility was due to male factors (214). Among women presenting with infertility, TSH levels were highest among women with ovulatory dysfunction and unknown causes of infertility and lower among those women with tubal infertility and whose infertility was due to male factors (215). In an uncontrolled study, 94 infertile women with hypothyroidism (TSH $>4.2 \mathrm{mU} / \mathrm{L}$ ) were treated with LT4 (25-150 $\mu \mathrm{g} / \mathrm{d})$ and 72 of them spontaneously conceived within 1 year (216). In a retrospective study, Yoshioka et al. (217) reported that $84.1 \%$ of infertile women with subclinical hypothyroidism (TSH $>3.0 \mathrm{mU} / \mathrm{L}$ ) successfully conceived and their infertility duration was shortened after LT4 therapy, suggesting that LT4 therapy may enhance fertility in patients with subclinical hypothyroidism. Importantly, whether or not LT4 treatment increases the likelihood of conception in subclinically hypothyroid women not undergoing ART has not been studied in controlled trials. Thus, insufficient data exist for 
recommending for or against routine LT4 therapy in subclinically hypothyroid, thyroid autoantibody-negative infertile women who are attempting conception but not undergoing ART.

\section{Question 24: Is thyroid autoimmunity linked to infertility in women?}

Limited evidence suggests that women with female-factor infertility are more likely to be TPOAb positive than agematched women who are not infertile, even if euthyroid (208). In addition, the prevalence of antithyroid antibodies may be higher in women with polycystic ovarian syndrome (PCOS) than in age-matched controls (218). Antithyroid antibodies are detectable in the ovarian follicles of women with thyroid autoimmunity and correlate with serum antibody levels (219), although whether such antibodies interfere with the fertilization potential of maturing oocytes is unknown. Among infertile women with PCOS, the presence of antithyroid antibodies has been associated with a decreased likelihood of developing ovarian follicles in response to treatment with clomiphene citrate (220).

\section{- RECOMMENDATION 16}

Evaluation of serum TSH concentration is recommended for all women seeking care for infertility.

Weak recommendation, moderate-quality evidence.

\section{- RECOMMENDATION 17}

LT4 treatment is recommended for infertile women with overt hypothyroidism who desire pregnancy.

Strong recommendation, moderate-quality evidence.

\section{- RECOMMENDATION 18}

Insufficient evidence exist to determine if LT4 therapy improves fertility in subclinically hypothyroid, thyroid autoantibody-negative women who are attempting natural conception (not undergoing ART). However, administration of LT4 may be considered in this setting given its ability to prevent progression to more significant hypothyroidism once pregnancy is achieved. Furthermore, low dose LT4 therapy $(25-50 \mu \mathrm{g} / \mathrm{d})$ carries minimal risk.

\section{Weak recommendation, low-quality evidence.}

\section{- RECOMMENDATION 19}

Insufficient evidence exists to determine if LT4 therapy improves fertility in nonpregnant, thyroid autoantibodypositive euthyroid women who are attempting natural conception (not undergoing ART). Therefore, no recommendation can be made for LT4 therapy in this setting.

No recommendation, insufficient evidence.

\section{Question 25: Is maternal subclinical hypothyroidism associated with worse ART outcomes?}

\section{Question 26: Does treatment of subclinically hypothyroid women improve ART outcomes?}

In a retrospective cohort study among women undergoing intrauterine insemination (IUI), the use of LT4 to treat subclinical hypothyroidism $(\mathrm{TSH}>2.5 \mathrm{mU} / \mathrm{L})$ was associated with higher pregnancy rates, and the use of LT4 to treat overt hypothyroidism with lower pregnancy rates, despite the fact that all LT4-treated patients had a serum $\mathrm{TSH}<2.5$ at the time of IUI (221). In a separate prospective cohort study of 1477 women undergoing IUI, preconception serum TSH in the range of $0.4-4.99 \mathrm{mU} / \mathrm{L}$ was not associated with IUI parameters, pregnancy rates, or live birth rates per cycle (222).

The majority of evidence suggests that IVF outcomes do not differ between women with serum TSH $<2.5 \mathrm{mU} / \mathrm{L}$ and those with very mild TSH elevations, defined as a TSH between 2.5 and $5 \mathrm{mU} / \mathrm{L}$. A retrospective cohort of $195 \mathrm{IVF}$ cycles that resulted in deliveries found that gestational age at birth was lower and mean birth weight was lower among women with preconception $\mathrm{TSH}>2.5 \mathrm{mU} / \mathrm{L}$ (223). In another retrospective cohort of 164 women undergoing IVF, clinical pregnancy rates were higher $(22 \%$ vs. $9 \%, p=0.045)$ in women with $\mathrm{TSH} \leq 2.5$ versus those with TSH $>2.5 \mathrm{mU} / \mathrm{L}$ (224). However, in a retrospective cohort of 1055 women undergoing their first IVF cycle, in age-adjusted analyses there was no difference in pregnancy, pregnancy loss, or delivery rates among women with serum TSH $<2.5 \mathrm{mU} / \mathrm{L}$ versus those with $\mathrm{TSH}<4.5 \mathrm{mU} / \mathrm{L}$ (225). Another recent retrospective study examined IVF outcomes in 627 women without known thyroid disease and concluded that the presence of prepregnancy subclinical hypothyroidism (serum TSH $>4.5 \mathrm{mU} / \mathrm{L}$ ) did not affect pregnancy rates, live birth rates, or miscarriage rates (226). A case-control study among women undergoing first IVF cycles found no difference in embryo quality among women with serum TSH 0.45-2.5 mU/L versus $2.5-4.5 \mathrm{mU} / \mathrm{L}$ (227). Another retrospective cohort similarly reported no associations between serum TSH in the range of $0.5-4.5 \mathrm{mU} / \mathrm{L}$ and IVF outcomes (228). Finally, in a retrospective study which aimed to control for embryo quality by limiting the cohort to 1599 euploid blastocyst transfer cycles, variations in serum TSH $\leq 2.5 \mathrm{mU} / \mathrm{L}$ were not associated with differences in implantation, pregnancy loss, or live birth rates (229).

However, data generally demonstrate that treatment of more significant elevations in TSH concentrations (although still classified as subclinical hypothyroidism) appears beneficial. A randomized clinical trial was conducted in women aged 20-40 years with subclinical hypothyroidism (serum TSH $>4.5 \mathrm{mU} / \mathrm{L}$ with normal FT4) who were undergoing IVF (230). A total of 64 women were randomized to treatment with LT4 $(50 \mu \mathrm{g} / \mathrm{d}$ starting at the time of initiation of ovarian stimulation, and titrated to maintain TSH $<2.5 \mathrm{mU} / \mathrm{L}$ in the first trimester in those women who did achieve pregnancy) versus placebo. The treated women had higher rates of clinical pregnancy, lower rates of miscarriage, and higher delivery rates. Another trial randomized 64 infertile women with subclinical hypothyroidism $(\mathrm{TSH}>4.2 \mathrm{mU} / \mathrm{L}$ with normal FT4) to treatment with $50 \mu \mathrm{g} / \mathrm{d}$ LT4 versus placebo and similarly reported higher pregnancy rates, lower pregnancy loss rates, and higher live birth rates in the treated women than in the control group (231). A recent meta-analysis pooled results of these trials with a third study (232) examining the effects of LT4 treatment for TPOAb-positive euthyroid women undergoing ART and concluded that although LT4 treatment did not have any effect on clinical pregnancy rates (pooled relative risk 1.75 [95\% CI $0.90-3.38]$ ), it did result in a higher delivery rate (pooled relative risk 2.76 [95\% CI 1.20-6.44]) (233).

Together, these data suggest that subclinical hypothyroidism likely affects ART in a dose-related fashion, such 
that impact worsens as TSH concentrations rise. It is also well known that TSH concentrations are variable and may differ by several milli-units per liter from week to week, despite no change in treatment (234). It therefore seems prudent to recommend treatment of subclinically hypothyroid women seeking pregnancy with ART for any TSH elevation $>2.5 \mathrm{mU} / \mathrm{L}$.

\section{- RECOMMENDATION 20}

Subclinically hypothyroid women undergoing IVF or intracytoplasmic sperm injection (ICSI) should be treated with LT4. The goal of treatment is to achieve a TSH concentration $<2.5 \mathrm{mU} / \mathrm{L}$.

\section{Strong recommendation, moderate-quality evidence.}

\section{Question 27: Is treated maternal hypothyroidism associated with worse ART outcomes compared to healthy controls?}

In a retrospective cohort study, among 240 women aged $\leq 37$ years undergoing first IVF retrieval cycles, 21 euthyroid (serum TSH $0.35-4.0 \mathrm{mU} / \mathrm{L}$ ) women with treated hypothyroidism had lower pregnancy rates than the women without thyroid disease (235). However, a subsequent case-control study including 137 LT4-treated hypothyroid women (mean serum TSH $1.6 \pm 0.7 \mathrm{mU} / \mathrm{L}$ ) and 274 age-matched euthyroid controls determined that the presence of adequately treated hypothyroidism was not associated with inferior rates of pregnancy, implantation, or live birth following IVF/ICSI (236). Thus, no conclusion can be drawn from these data.

\section{Question 28: Is maternal antithyroid Ab positivity associated with poorer outcomes following ART?}

Studies examining ART outcomes in thyroid autoantibodypositive and -negative women have enrolled heterogeneous populations with differing underlying etiologies for infertility. Some, but not all studies have been limited to women who were euthyroid. Different ART/IVF protocols have also been employed in different places and over time. A meta-analysis of prospective cohort studies suggests that pregnancy rates following IVF do not differ between Ab-positive and Abnegative women, but as discussed previously (see Section V), that risk of pregnancy loss is higher in women with thyroid autoantibodies positivity (237). However, a recent retrospective study examined IVF outcomes in 627 women without known thyroid disease and concluded that the presence of prepregnancy thyroid autoimmunity did not affect pregnancy rates, live birth rates, or pregnancy loss rates (226). Similarly, two recent retrospective cohorts reported no differences in pregnancy, pregnancy loss, or live birth rates in thyroid Ab-positive versus thyroid Ab-negative euthyroid women undergoing IVF with ICSI $(238,239)$. Separately, a prospective cohort study found no differences in outcomes of IVF with ICSI in TPOAb-positive and/or TgAb-positive versus euthyroid women with Ab negativity (240). By contrast, a recent retrospective IVF cohort found that fertilization, implantation, and pregnancy rates were lower in 90 antithyroid Ab-positive compared to $676 \mathrm{Ab}$-negative women, but thyroid function was not reported in either group (241).

\section{Question 29: Does treatment of antithyroid Ab-positive euthyroid women improve ART outcomes?}

Based on a single small randomized clinical trial and one retrospective cohort, LT4 treatment for thyroid Ab-positive women without thyroid dysfunction undergoing IVF does not appear to improve outcomes $(232,242)$. Two small trials suggest the potential for improved pregnancy rates in thyroid Ab-positive infertile women who are treated with glucocorticoids prior to ART $(243,244)$. In the trial by Litwicka et al. (243), 60 euthyroid (TSH $<2.5 \mathrm{mU} / \mathrm{L}$ ) TPOAb- and/or TgAbpositive women undergoing IVF were randomized to prednisolone $5 \mathrm{mg} / \mathrm{d}$ starting from the day of oocyte retrieval and continuing through the first trimester. There were higher overall pregnancy $(60 \%$ vs. $30 \%, p=0.02)$ and live birth $(46.6 \%$ vs. $20 \%, p=0.05)$ rates in the treated versus untreated women. Turi and colleagues (244) randomized 48 TPOAbpositive infertile women to prednisone versus placebo for 4 weeks before IUI. The pregnancy rate was $33.3 \%$ in treated women compared to $8.4 \%$ in the placebo group $(p=0.03)$. Pregnancy loss rates were not significantly different between the two groups. Although these small trials appear promising, the risks of corticosteroid use in early pregnancy are not well understood (245).

\section{- RECOMMENDATION 21}

Insufficient evidence exists to determine whether LT4 therapy improves the success of pregnancy following ART in TPOAb-positive euthyroid women. However, administration of LT4 to TPOAb-positive euthyroid women undergoing ART may be considered given its potential benefits in comparison to its minimal risk. In such cases, $25-50 \mu \mathrm{g}$ of LT4 is a typical starting dose.

Weak recommendation, low-quality evidence.

\section{- RECOMMENDATION 22}

Glucocorticoid therapy is not recommended for thyroid autoantibody-positive euthyroid women undergoing ART.

Weak recommendation, moderate-quality evidence.

\section{Question 30: Does ovarian hyperstimulation alter thyroid function?}

Although multiple ART protocols are currently in use, protocols for IVF or IVF with ICSI typically begin by inducing controlled ovarian hyperstimulation through the use of gonadotrophins, gonadotrophin-releasing hormone analogues, or gonadotrophin-releasing hormone antagonists in combination with gonadotrophins. Follicular development is monitored by ultrasound, and when leading follicles are large enough, hCG is administered to produce fully mature oocytes. Controlled ovarian hyperstimulation causes serum estradiol to rapidly rise to supraphysiologic levels (4000$6000 \mathrm{ng} / \mathrm{L}$ ), comparable to those detected in late pregnancy (246). These hormonal manipulations may alter thyroid function. The presumed mechanism for this effect relates to the rise in TBG associated with high estrogen levels, which reduce free thyroid hormone concentrations and in turn feed back to cause serum TSH elevations. In addition, the administered hCG can directly stimulate thyroidal TSH receptors, causing increases in thyroid hormone and subsequent 
decreases in TSH. A systematic review (247) found inconsistent effects of ovarian stimulation on serum thyroid hormones. For example, during and up to 1 month after ovarian stimulation serum TSH was increased in three of five studies, while FT4 was increased in two studies, decreased in one study, and unchanged in another. In some women (248-250), serum TSH levels increased to $\geq 2.5 \mathrm{mU} / \mathrm{L}$ following ovarian stimulation. Gracia and colleagues (251) reported increases in both serum TSH and FT4 during ovarian stimulation that peaked 1 week after hCG administration, with $44 \%$ of the women with baseline $\mathrm{TSH} \leq 2.5 \mathrm{mU} / \mathrm{L}$ having increases to $>2.5 \mathrm{mU} / \mathrm{L}$. Reinblatt et al. (252) reported that serum TSH increased in parallel with serum estradiol during the course of ovarian stimulation, with more marked $\mathrm{TSH}$ increases seen among TPOAb-positive women.

Ovarian hyperstimulation syndrome (OHSS) is a complication of controlled ovarian hyperstimulation in which increased vascular permeability results in fluid shifts from intravascular to third space compartments. Mild OHSS, manifested by mild ascites and abdominal symptoms, occurs in up to $20 \%-33 \%$ of all IVF cycles. Severe OHSS, which occurs in $0.1 \%-2 \%$ of IVF cycles, may be associated with thrombosis and respiratory distress, and is occasionally fatal (253). A recent case report described a patient who had subclinical hypothyroidism prior to IVF that was adequately treated with LT4. The patient rapidly developed overt hypothyroidism in the setting of severe OHSS (254). However, Poppe et al. (246) prospectively studied thyroid function in women undergoing controlled ovarian hyperstimulation and found no differences in serum TSH or FT4 levels between 25 women who developed OHSS and 52 women who did not.

Among women with adequately treated hypothyroidism (i.e., receiving LT4 therapy), ovarian stimulation has been reported to induce hypothyroidism by the time of egg retrieval (255). In one cohort study, increases in serum TSH during ovarian stimulation were more marked in LT4-treated women than in women without underlying hypothyroidism 1 week after hCG administration. However, TSH levels no longer differed between the groups by the time of pregnancy testing (251). In a prospective cohort study in 72 treated hypothyroid women with serum $\mathrm{TSH}<2.5 \mathrm{mU} / \mathrm{L}$ prior to ovarian stimulation, serum TSH rose sequentially from $1.7 \pm 0.7 \mathrm{mU} / \mathrm{L}$ at baseline to $2.9 \pm 1.3 \mathrm{mU} / \mathrm{L}$ at the time of $\mathrm{hCG}$ administration, and to $3.2 \pm 1.7 \mathrm{mU} / \mathrm{L} 16$ days after hCG, with serum hCG $>2.5 \mathrm{mU} / \mathrm{L}$ in $46(64 \%)$ of subjects by the time hCG was administered (256). A retrospective cohort study demonstrated that among treated hypothyroid women who undergo IVF, 84\% required LT4 dose increases, most within the first 5-7 weeks of gestation (257). However, a small prospective study found that treated hypothyroid patients who conceived with the help of gonadotrophin therapy did not require larger LT4 dose increases than treated hypothyroid women who conceived spontaneously (258).

\section{- RECOMMENDATION 23}

When possible, thyroid function testing should be performed either before or 1-2 weeks after controlled ovarian hyperstimulation because results obtained during the course of controlled ovarian stimulation may be difficult to interpret.

Weak recommendation, moderate-quality evidence.

\section{- RECOMMENDATION 24}

In women who achieve pregnancy following controlled ovarian hyperstimulation, TSH elevations should be treated according to the recommendations outlined in Section VII. In nonpregnant women with mild TSH elevations following controlled ovarian stimulation, serum TSH measurements should be repeated in 2-4 weeks because levels may normalize.

\section{Weak recommendation, moderate-quality evidence.}

\section{HYPOTHYROIDISM AND PREGNANCY}

Primary overt maternal hypothyroidism is generally defined as the presence of an elevated TSH and a decreased serum FT4 concentration during gestation, with both concentrations outside the (trimester-specific) reference ranges. In very rare cases, it is important to exclude other causes of abnormal thyroid function such as TSH-secreting pituitary tumors, thyroid hormone resistance, or central hypothyroidism with biologically inactive TSH. Several investigations report that at least $2 \%-3 \%$ of healthy, nonpregnant women of childbearing age have an elevated serum TSH $(259,260)$. The prevalence may be higher in areas of iodine insufficiency. When iodine nutrition is adequate, the most frequent cause of hypothyroidism is autoimmune thyroid disease (Hashimoto's thyroiditis). Therefore, not surprisingly, thyroid autoantibodies can be detected in approximately 30\%-60\% of pregnant women with an elevated TSH concentration $(19,260,261)$.

In the 2011 ATA guidelines, the upper reference limit for serum TSH concentration during pregnancy was defined as $2.5 \mathrm{mU} / \mathrm{L}$ in the first trimester, and $3.0 \mathrm{mU} / \mathrm{L}$ in the second and third trimesters. These cutoffs were predominantly based on the published reference ranges obtained from six pregnancy studies together comprising a total cohort of approximately 5500 subjects $(12,13,18,262-264)$. Since that publication, additional much larger cohorts have published center-specific and trimester-specific pregnancy reference ranges. These analyses combine data from over $60,000 \mathrm{sub}-$ jects $(17,24,265-268)$. Importantly, this larger analysis demonstrates substantial population differences in the TSH upper reference limit (Table 4) (17,24,142,262,264-274). These differences may be partly attributable to differences in the iodine status between populations as well as the TSH assays used for analysis. However, these data also demonstrate important influences of BMI, geography, and ethnicity upon "normalcy" of TSH concentrations in pregnant women. In summary, substantial variation exists between populations, with many recent investigations confirming a more liberal upper TSH reference range in healthy pregnant women with no thyroid disease (269). Equally important, recent studies have also demonstrated an important additive influence of TPOAb positivity upon maternal thyroid status. Increasingly, there appears to be a greater risk for adverse events in women who are TPOAb positive compared to those who are TPOAb negative, even when thyroid function is identical. The reasons for this difference remain unclear. As described in Section V, some studies suggest that TPOAb-positive euthyroid women may be at increased risk for adverse clinical outcomes not observed in TPOAb-negative comparators (19). As a consequence, it is difficult to precisely define a universal TSH cutoff above which LT4 therapy should be initiated for all pregnant women. Rather, decisions about LT4 
treatment must be based upon both measurement of thyroid function and TPOAb status.

\section{Question 31: What is the definition of hypothyroidism in pregnancy?}

Elevations in serum TSH concentrations during pregnancy should ideally be defined using pregnancy- and populationspecific reference ranges. It is important to note that detection of an increased TSH concentration is not always synonymous with decreased FT4 concentrations. Frequently, elevated maternal TSH is detected when FT4 concentrations are normal. Conversely, low FT4 concentrations can be detected despite normal TSH concentrations. The latter situation is referred to as isolated hypothyroxinemia. Excepting the very rare scenarios noted above, serum TSH measurement remains the principal determinant of maternal thyroid status at the present time and should be used to guide treatment decisions and goals. Because substantial differences exist in the upper reference limit for TSH between different populations (Table 4), each practitioner and hospital should ideally seek to determine their own trimesterspecific reference ranges, obtained from analysis of healthy, TPOAb-negative, and iodine-sufficient women. However, the task force recognizes that this goal is frequently not feasible. Therefore, this guideline also proposes a stepwise approach to evaluation, providing a practical guide for clinicians.

\section{- RECOMMENDATION 25}

In the setting of pregnancy, maternal hypothyroidism is defined as a TSH concentration elevated beyond the upper limit of the pregnancy-specific reference range.

\section{Strong recommendation, high-quality evidence.}

\section{- RECOMMENDATION 26}

The pregnancy-specific TSH reference range should be defined as follows:

$\circ$ When available, population- and trimester-specific reference ranges for serum TSH during pregnancy should be defined by a provider's institute or laboratory and should represent the typical population for whom care is provided. Reference ranges should be defined in healthy TPOAb-negative pregnant women with optimal iodine intake and without thyroid illness. Strong recommendation, high-quality evidence.

$\circ$ When this goal is not feasible, pregnancy-specific TSH reference ranges obtained from similar patient populations and performed using similar TSH assays should be substituted (Table 4).

Strong recommendation, high-quality evidence.

- If internal or transferable pregnancy-specific TSH reference ranges are not available, an upper reference limit of $\sim 4.0 \mathrm{mU} / \mathrm{L}$ may be used. For most assays, this limit represents a reduction in the nonpregnant $\mathrm{TSH}$ upper reference limit of $\sim 0.5 \mathrm{mU} / \mathrm{L}$.

Strong recommendation, moderate-quality evidence.

\section{Question 32: How is isolated hypothyroxinemia defined in pregnancy?}

Isolated hypothyroxinemia is typically defined as a FT4 concentration in the lower 2.5 th- 5 th percentile of a given population in conjunction with a normal maternal TSH concentration.

\section{Question 33: What adverse outcomes are associated with overt hypothyroidism during pregnancy?}

Overt maternal hypothyroidism has consistently been shown to be associated with an increased risk of adverse pregnancy complications (275) as well as detrimental effects upon fetal neurocognitive development (276). Specific adverse outcomes associated with overt maternal hypothyroidism include increased risks of premature birth, low birth weight, pregnancy loss, and lower offspring IQ. Abalovich et al. (277) demonstrated that women with overt hypothyroidism carry an estimated $60 \%$ risk of fetal loss when not adequately treated. Separately, Leung et al. (278) demonstrated a $22 \%$ risk of gestational hypertension in pregnant women with overt maternal hypothyroidism. Finally, Allan et al. (260) similarly described an increased risk of fetal death among pregnant women with overt disease. Together, these data demonstrate a clear association between overt maternal hypothyroidism and risk to the maternal-fetal unit.

\section{Question 34: What adverse outcomes are associated with subclinical hypothyroidism during pregnancy?}

Subclinical hypothyroidism is variably associated with an increased risk of adverse pregnancy outcomes in most, but not all studies, partly because separate studies use differing cutoffs to define an elevated TSH concentration. Also, many studies do not account for TPOAb status. Table 5 provides an overview of all available observational and prospective studies (inclusive of at least 400 subjects) investigating the effect of maternal subclinical hypothyroidism upon pregnancy $(19,192,193$, 259,279-285,287-289,291,294,295,306,314,318). Separately, Table 6 describes the additive adverse impact of TPOAb status upon maternal hypothyroidism $(19,192,279,284,287-289,291$, 295,314).

For the purposes of discussion, the investigations that have studied the association between elevated maternal TSH concentration and adverse clinical endpoints can be broadly grouped into three categories below based upon adverse endpoints. These include adverse effects on pregnancy outcome (i.e., pregnancy loss), adverse perinatal outcomes (i.e., premature delivery, hypertensive disorders), and adverse neurocognitive outcomes (IQ) in offspring.

Pregnancy loss. Early fetal loss naturally occurs in approximately $30 \%$ of pregnancies. Since the majority of pregnancy losses occur even before pregnancy is clinically recognized, pregnancy loss is a difficult study endpoint (158). Nevertheless, different studies have suggested a relationship between higher levels of maternal TSH and pregnancy loss. Negro et al. (286) reported a significantly higher pregnancy loss rate in TPOAb-negative women with TSH concentrations between 2.5 and $5.0 \mathrm{mU} / \mathrm{L}$ compared to those with TSH concentrations below $2.5 \mathrm{mU} / \mathrm{L}$ (6.1\% vs. $3.6 \%)$. Similarly, Benhadi et al. (287) performed a prospective cohort study investigating the risk of pregnancy loss (defined as miscarriage or fetal or neonatal death) in 2497 Dutch women. In this cohort of pregnant women without overt hypothyroidism, the 


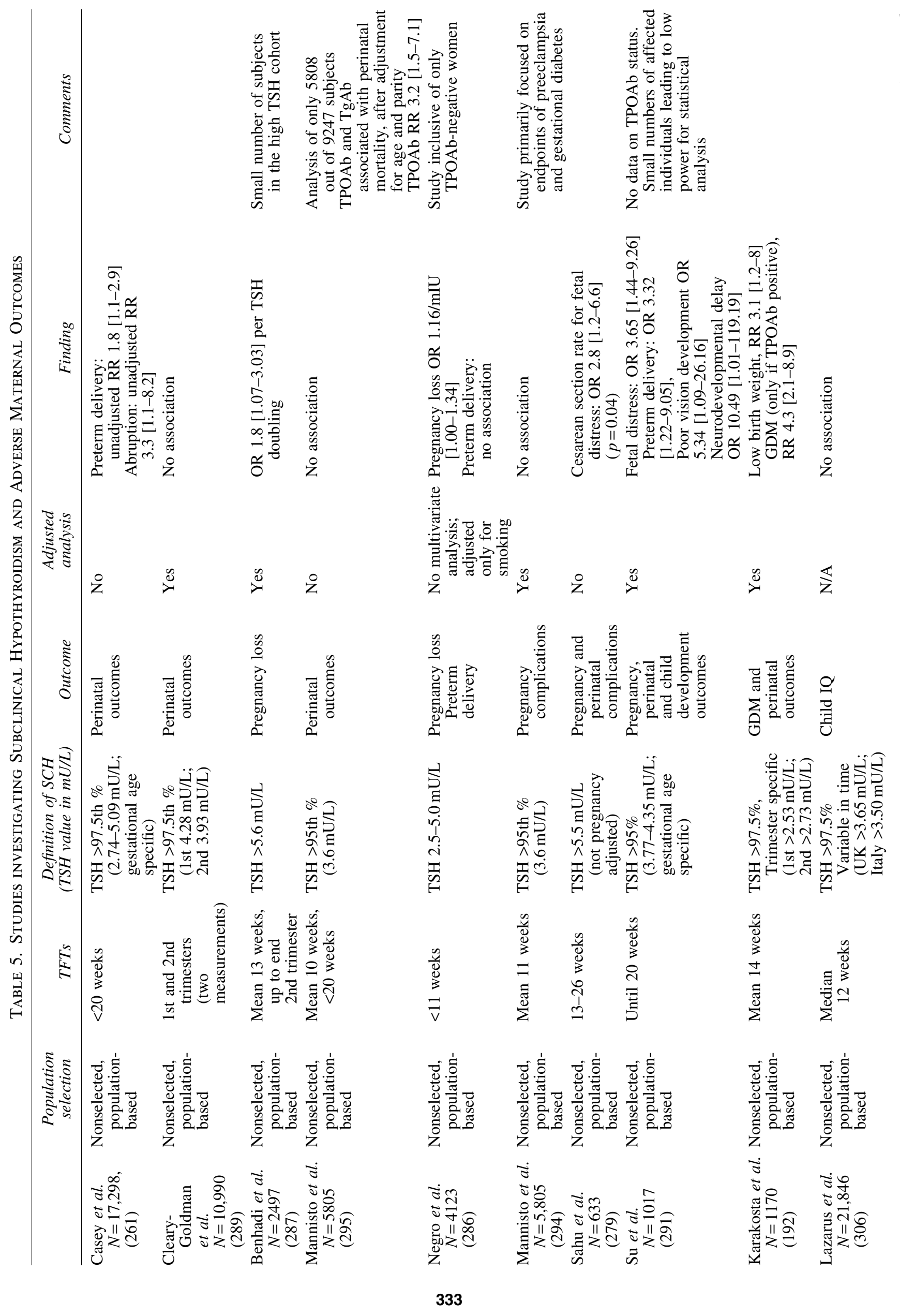




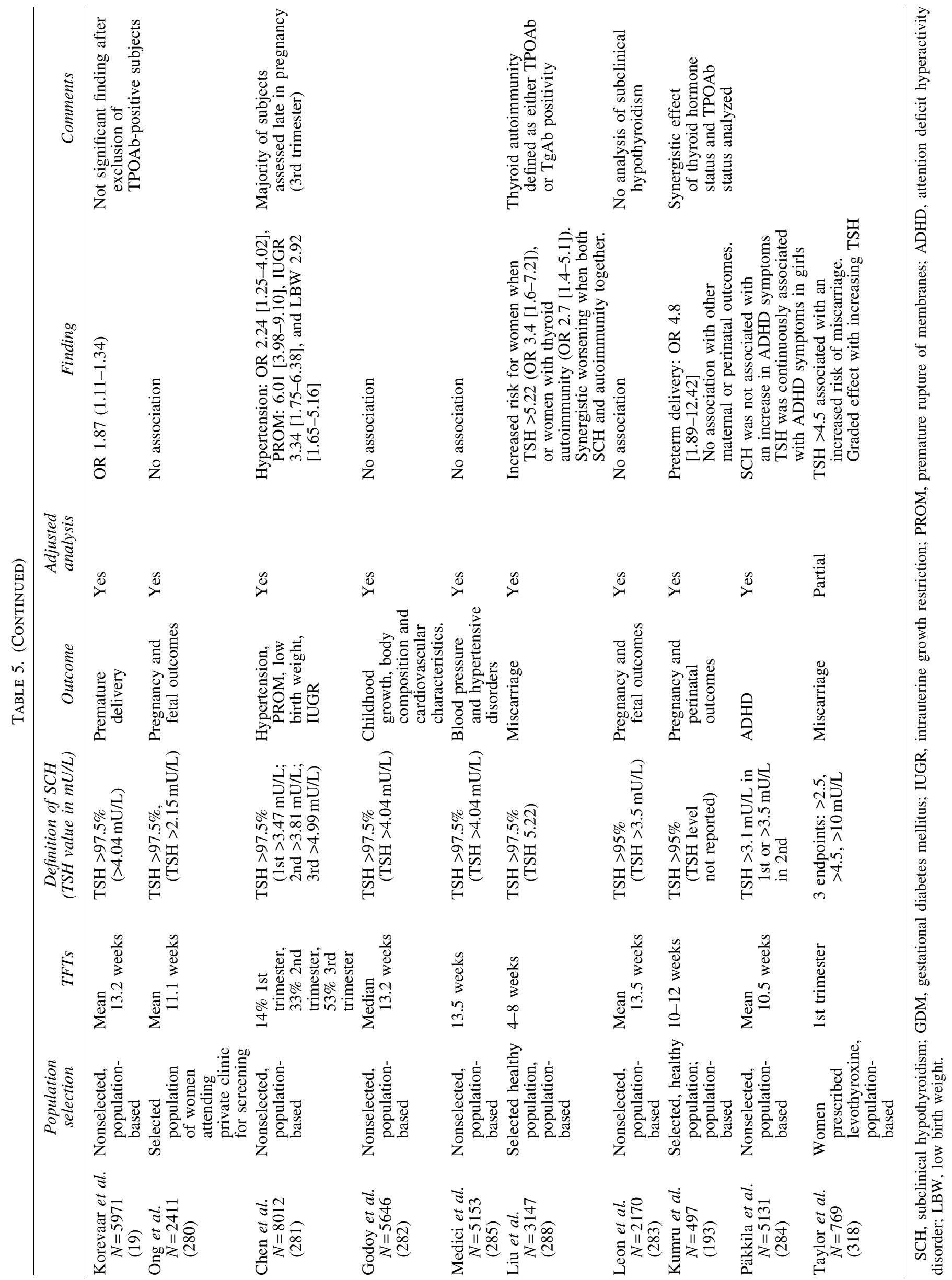



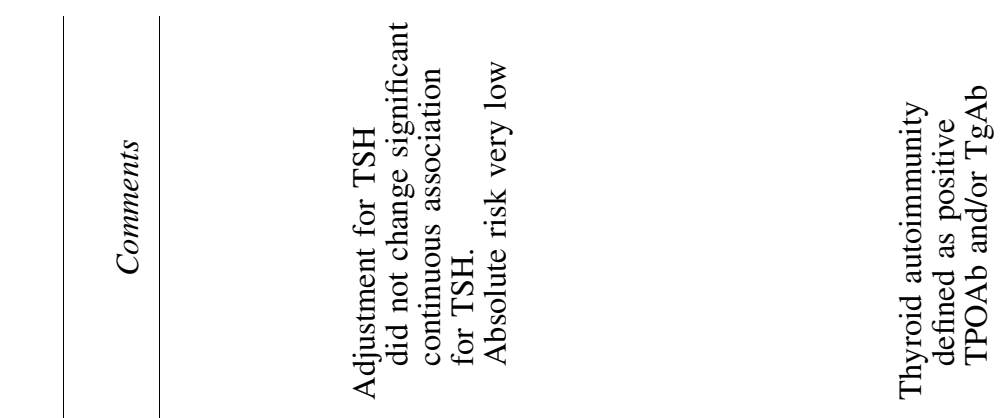

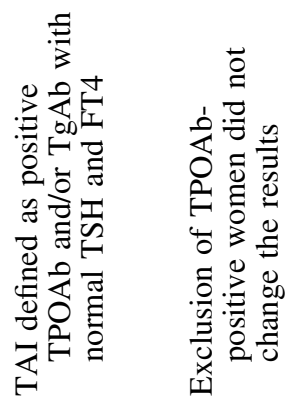

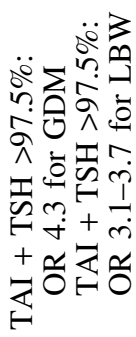

0
0
0
0
0
0
0
0
0
0

i

ซै

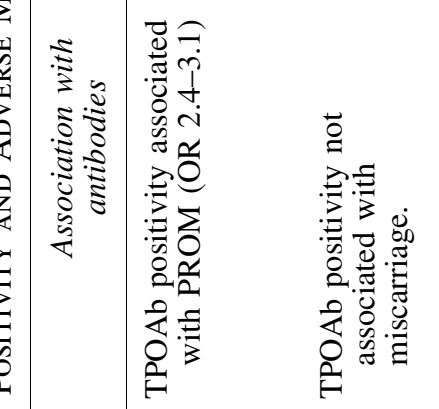

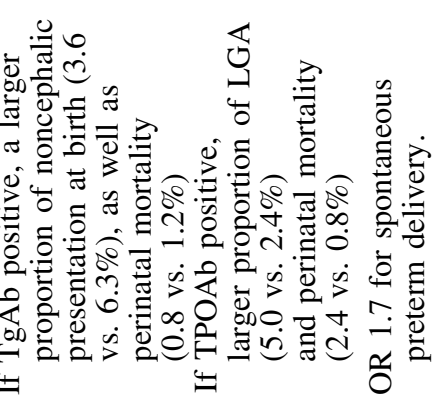

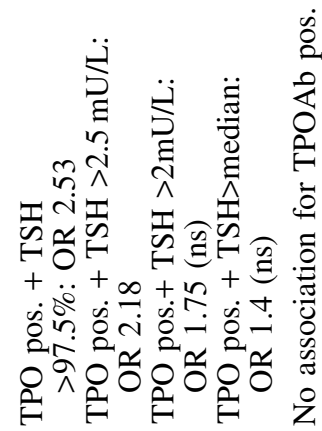

0
0
0
0
0
0
0
0
0

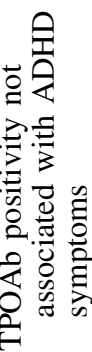

$\stackrel{8}{\varnothing}$

$\stackrel{3}{*}$

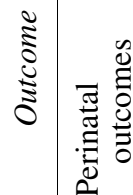

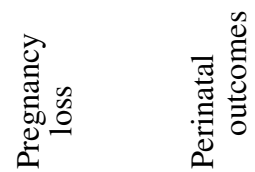

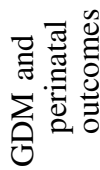

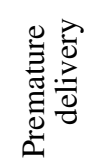

$\stackrel{\circ}{*}$

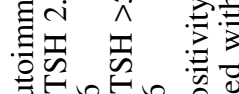

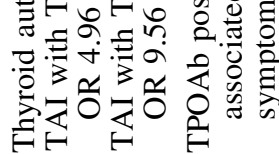

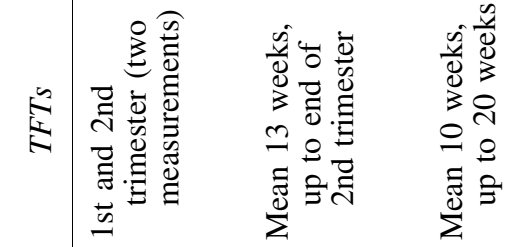
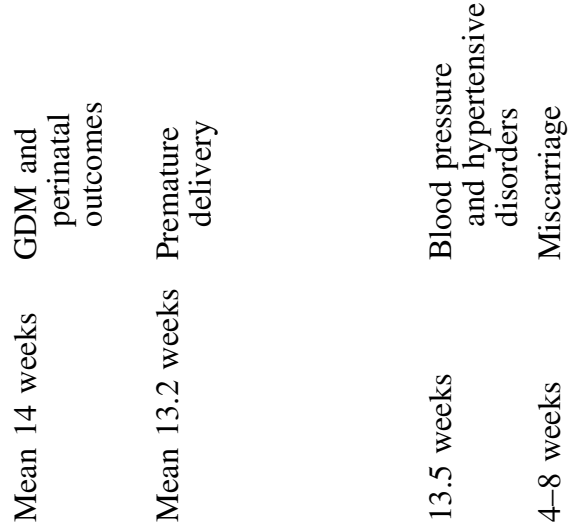

$\stackrel{0}{7}$
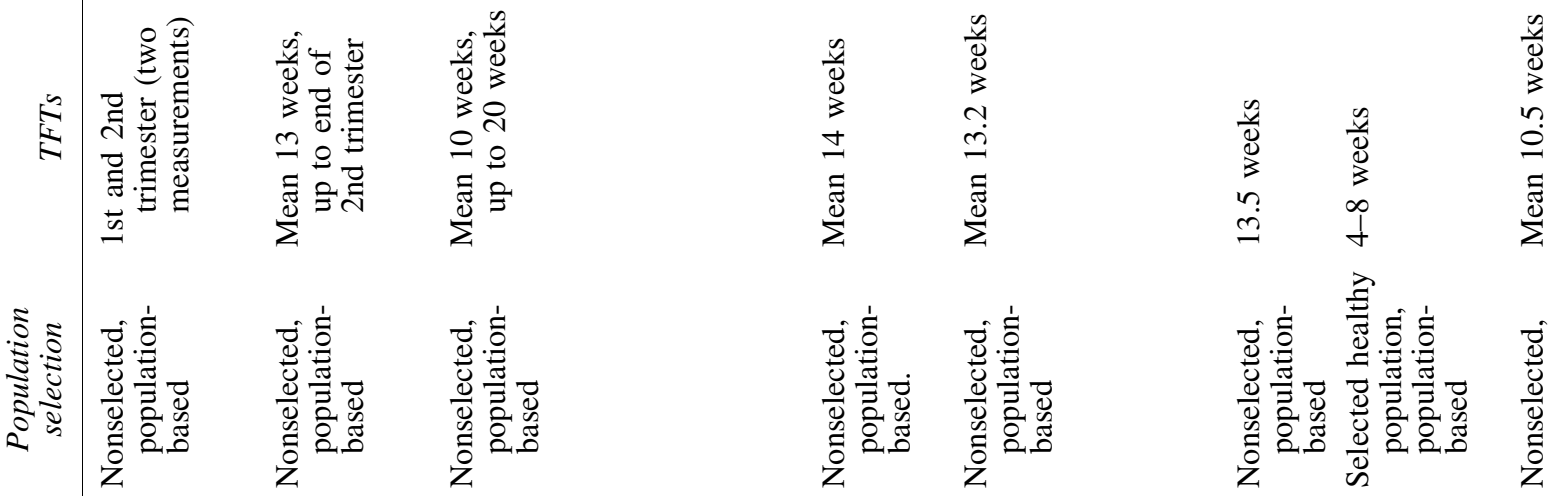

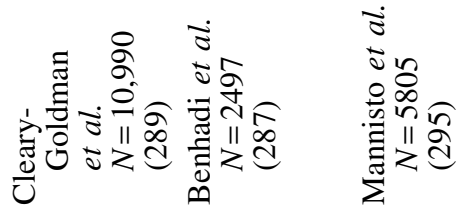
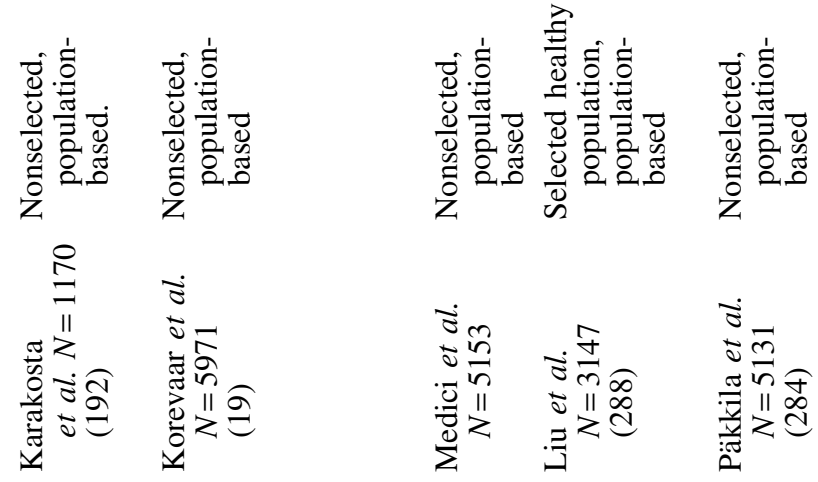

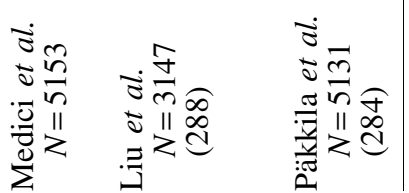


risk of child loss increased with higher levels of maternal $\mathrm{TSH}$, although results should be interpreted with caution given the very small number of 27 cases studied as well as the heterogeneity of the study's endpoint. In an Australian cohort, early-pregnancy TSH levels $>95$ th percentile were associated with an increased risk of miscarriage (OR 3.66) although subclinical and overt hypothyroid cases were pooled (292). A separate retrospective study that determined thyroid parameters in early-pregnancy samples obtained from 202 pregnancies that subsequently miscarried showed higher mean TSH and lower FT4 concentrations as well as a higher prevalence of TSH concentrations $>97.5$ th percentile and FT4 concentrations $<2.5$ th percentile compared to 3592 normal pregnancies (137). More recently, Liu and colleagues (288) demonstrated a graded increase in miscarriage risk as maternal TSH concentrations increased. This effect was augmented by the presence of TPOAb positivity.

Negro et al. (286) published data suggesting that subclinical hypothyroidism increases the risk of pregnancy complications in TPOAb-positive women. In this prospective trial of more than 4600 subjects, women were randomized to universal screening versus case finding (high-risk screening) during pregnancy, with subsequent LT4 treatment of TPOAb-positive women with TSH $>2.5 \mathrm{mU} / \mathrm{L}$. Low-risk women in the unscreened group had serum collected and stored for analysis post partum. Within the subset of women classified as low risk for hypothyroidism, treatment of TPOAb-positive women with TSH $>2.5 \mathrm{mU} / \mathrm{L}$ resulted in a significant reduction in a composite endpoint of pregnancy complications compared to no treatment. The composite endpoint remains a significant study limitation because many variables were subjective in nature. Furthermore, it is critical to note that the primary study endpoint was nonsuperior, showing no benefit of universal screening and treatment (286) compared with screening of high-risk women only, because the primary, predefined endpoint analyzed the effects of LT4 treatment on both low-risk and high-risk subjects together. Importantly, all high-risk women in the study were tested and treated for elevated TSH values. Therefore, when combining both groups, the treatment effect on the low-risk group was diluted, leading to the conclusion of no superiority of universal screening. As such, this study should be viewed as providing important and provocative data that are worthy of further study (286). However, its conclusion that universal screening did not confer a benefit, combined with the difficulty in drawing conclusions from a composite endpoint, makes it challenging to translate into clinical practice.

Premature delivery and other pregnancy complications. The largest study investigating the association of maternal hypothyroidism and premature delivery was performed by Casey et al. (261) in a cohort of 17,298 pregnant women presenting for prenatal care. Subclinical hypothyroidism was associated with an increased risk of premature delivery at $<34$ weeks ( $4 \%$ vs. $2.5 \%, p=0.01$ ), but not with premature delivery at $<32$ weeks $(2.5 \%$ vs. $1 \%, p=0.07)$ or at $<36$ weeks ( $7 \%$ vs. $6 \%, p=0.39$ ). This lack of continuous effect raises questions about the 34-week finding. A later study by ClearyGoldman et al. (289) showed no association of an elevated TSH with prematurity at $<37$ weeks. Separately, other studies have also investigated this potential adverse relationship, albeit with conflicting results (192,260,266,286,290-292).
This variation can in part be explained by some studies pooling overt and subclinical hypothyroid cases together $(260,292)$, while others used different TSH cutoff values $(286,290)$, and yet others enrolled a very limited number of subjects $(192,291)$. A recent study compared the value of using population-based reference range limits ( $\mathrm{TSH}>97.5$ percentile, defined as $4.0 \mathrm{mU} / \mathrm{L}$ ) versus a fixed TSH cutoff of $2.5 \mathrm{mU} / \mathrm{L}$ (19). While a $\mathrm{TSH}>2.5 \mathrm{mU} / \mathrm{L}$ was not associated with premature delivery, 1.9 and 2.5 times increased risks of prematurity at $<37$ and $<34$ weeks, respectively, were observed among women with TSH $>4.0 \mathrm{mU} / \mathrm{L}$. Interestingly, this association no longer persisted after exclusion of TPOAb-positive women or women with comorbidities. This shows that these factors may be important confounders in various studies and underlines the importance of performing in-depth analyses of observed associations.

The majority of large studies focusing upon pre-eclampsia and hypertensive disorders, with one exception one (293), did not find associations of other pregnancy complications with elevated TSH $(260,289,294)$. Mannisto and colleagues $(294,295)$ evaluated the relationship between pregnancy outcomes and thyroid function tests obtained at 12 weeks gestation in 5805 women. No adverse association between thyroid function and perinatal mortality was noted. A separate large study investigating the relationship between subclinical hypothyroidism and birth weight showed no effect on very low $(<2500 \mathrm{~g})$ or high ( $>4000 \mathrm{~g}$ ) birth weights (289). This finding was also confirmed by Mannisto et al. (295). A recent meta-analysis broadly analyzed pregnancy outcomes in relation to maternal thyroid status (296). The authors found an increasing risk of pregnancy complications (pregnancy loss, preterm delivery, and placental abruption) in relation to maternal subclinical hypothyroidism during early pregnancy, although subclinical hypothyroidism was variably defined across studies (296).

Together, despite some differences in study design, biochemical cutoffs applied and slightly differing endpoints, the above studies overall indicate an increasing risk of pregnancy-specific complications, most notably pregnancy loss and preterm delivery, in relation to elevated maternal TSH concentrations. Importantly, however, this effect is exacerbated by the presence of elevated TPOAb, such that any additive risk is apparent in TPOAb-positive women when TSH exceeds $2.5 \mathrm{mU} / \mathrm{L}$. However, in TPOAb-negative women similar adverse risk is not consistently apparent until maternal TSH exceeds 5-10 mU/L.

Adverse neurocognitive effect on the offspring. The detrimental effects of maternal thyroid hypofunction on fetal neurocognitive development are less clear. In support of an adverse impact attributable to maternal hypothyroidism, data from a large case-control study demonstrated a seven-point reduction in IQ among children born to untreated overtly hypothyroid women compared to euthyroid controls (276). Findings also supported a delay in motor skill development, language development, and attention at 7-9 years of age. Subsequent studies have shown similar impact on children born to women with isolated hypothyroxinemia, $(19,283$, 297-304,314,317). Separately, three small studies analyzing only TPOAb positivity appear to similarly show an effect on neurocognitive outcome in the offspring but need to be confirmed in larger samples $(199,201,305)$.

In contrast, the Controlled Antenatal Thyroid Screening (CATS) study was a large prospective RCT investigating the 
benefit of population screening for elevated TSH concentrations and low FT4 concentrations in pregnant women. This study demonstrated no improvement in cognitive function when children of treated hypothyroid or hypothyroxinemic mothers were evaluated at 3 years of age. In this cohort, detection of either an elevated TSH or low FT4 triggered initiation of $150 \mu \mathrm{g} / \mathrm{d}$ LT4 therapy, at a mean of 13 weeks and 3 days gestation (306). Preliminary results of another large multicenter, randomized, controlled trial, the Randomized Trial of Thyroxine Therapy for Subclinical Hypothyroidism or Hypothyroxinemia Diagnosed During Pregnancy, have recently become available. This study screened 97,226 pregnant women in order to randomize 677 women with subclinical hypothyroidism and 526 women with isolated maternal hypothyroxinemia to LT4 treatment versus placebo at a mean time point of 17 weeks. Similarly, this study demonstrated no significant effect of treatment on offspring IQ at the age of 5 years (21). Other smaller studies support this conclusion (307-309).

Taken together, these prospective results provide insufficient evidence to conclude that treatment of subclinical hypothyroidism is associated with improved neurocognitive outcomes in offspring.

It should be noted, however, that the lack of positive data does not rule out a potential harmful effect, nor does it rule out the theoretical effectiveness of any intervention. It is important to note that such studies are highly difficult to complete and often enroll subjects with great heterogeneity with regard to important study parameters. For example, the timing of LT4 intervention during gestation likely plays an important role in the effect of any intervention. The two randomized controlled studies described above initiated LT4 treatment only at the completion of the first trimester or later-which may be too late to significantly impact neurodevelopment. Animal studies have suggested any window of opportunity is likely earlier in gestation (310,311). Similarly, the duration and severity of maternal hypothyroidism are likely important, yet virtually never controlled for, because all studies include only a single baseline measurement of TSH concentration during pregnancy. It therefore must again be emphasized that overt maternal hypothyroidism during pregnancy should be considered dangerous, and logic suggests that moderate (or even mild) maternal hypothyroidism may similarly impart risk. What remains uncertain is the nuanced understanding of how this risk is reduced or abated as the extent of maternal hypothyroidism is normalized or other variables are modified. This point should be emphasized as we consider that the most common cause of maternal hypothyroidism has dramatically changed over the last century. Formerly, severe iodine deficiency was common, while more recently, the principal cause of maternal hypothyroidism is maternal Hashimoto's disease. These disorders are physiologically different, though both may impart a similar phenotype demonstrating elevated maternal TSH concentrations.

\section{Question 35: What adverse outcomes are associated with isolated hypothyroxinemia in pregnancy?}

Pop and colleagues (312) initially reported a decrease in psychomotor test scores among offspring born to women with FT4 indices in the lowest 10th percentile, despite having normal serum TSH concentrations. Similarly, Li et al. (199) observed a reduction in IQ among offspring of mothers suffering from either hypothyroidism or isolated hypothyroxinemia during the first trimester. In recent years, additional prospective, nonrandomized studies have similarly reported adverse child outcomes in children born to mothers with isolated hypothyroxinemia $(19,192,193,259,279,280-284$, 287-289,291,294,295,306,313,314,318) (see Tables 5 and 6). Adverse outcomes include lower IQ, language delay, worsened motor function, smaller head circumference, and an increased risk of autism. These data are derived from different populations across the world (China, Belgium, the Netherlands, Spain) with known differences in iodine status. In contrast to those studies investigating the association of elevated TSH concentrations with adverse pregnancy outcome, however, very few studies have investigated isolated hypothyroxinemia and adverse pregnancy outcomes (see Table 7), excepting birth weight (314-316) and premature delivery (19). Available data suggest an association with higher birth weight and higher risk of premature delivery. Interestingly, many large-scale studies demonstrate that the populations of women with elevated TSH concentrations are generally exclusive from those identified with low FT4 concentrations. For example, in the CATS study, approximately the same proportion of screened mothers were identified in the hypothyroxinemic and subclinically hypothyroid groups, with little overlap.

Overall, available evidence appears to show an association between hypothyroxinemia and cognitive development of the offspring, with uncertain effects on prematurity (314-316) and low birth weight (19). However, no studies exist in which LT4 administration has been shown to ameliorate such harmful effects. In the CATS trial (303), 499 women were identified with low FT4 concentrations and 242 randomized to treatment with LT4. This intervention failed to demonstrate any improvement in neurocognitive outcomes in the offspring at 3 years of age. However, women in this study received a fairly high dose $(150 \mu \mathrm{g})$ of LT4 treatment and $10 \%$ needed a dose reduction because of biochemical or clinical signs of overtreatment. This is interesting because a recent study by Korevaar et al. (317) showed that both low and high FT4 concentrations may be associated with a decrease in child IQ and reduction of cerebral gray matter volume as assessed by magnetic resonance imaging.

\section{Question 36: Should women with overt hypothyroidism be treated in pregnancy?}

As mentioned above, numerous retrospective and casecontrol studies confirm the detrimental effects of overt hypothyroidism on both pregnancy and fetal health (318). A recent retrospective study of more than 1000 pregnant women on chronic LT4 replacement, showed that the risk of pregnancy loss increased proportionally to the degree of TSH elevation, with no increased risk associated with TSH normalization (318). Although no prospective, randomized investigation of LT4 intervention to improve obstetric outcomes or child development has occurred in pregnant women with overt hypothyroidism, such an investigation would be unethical to complete. Nonetheless, available data confirm the benefits of treating severe hypothyroidism during pregnancy. 


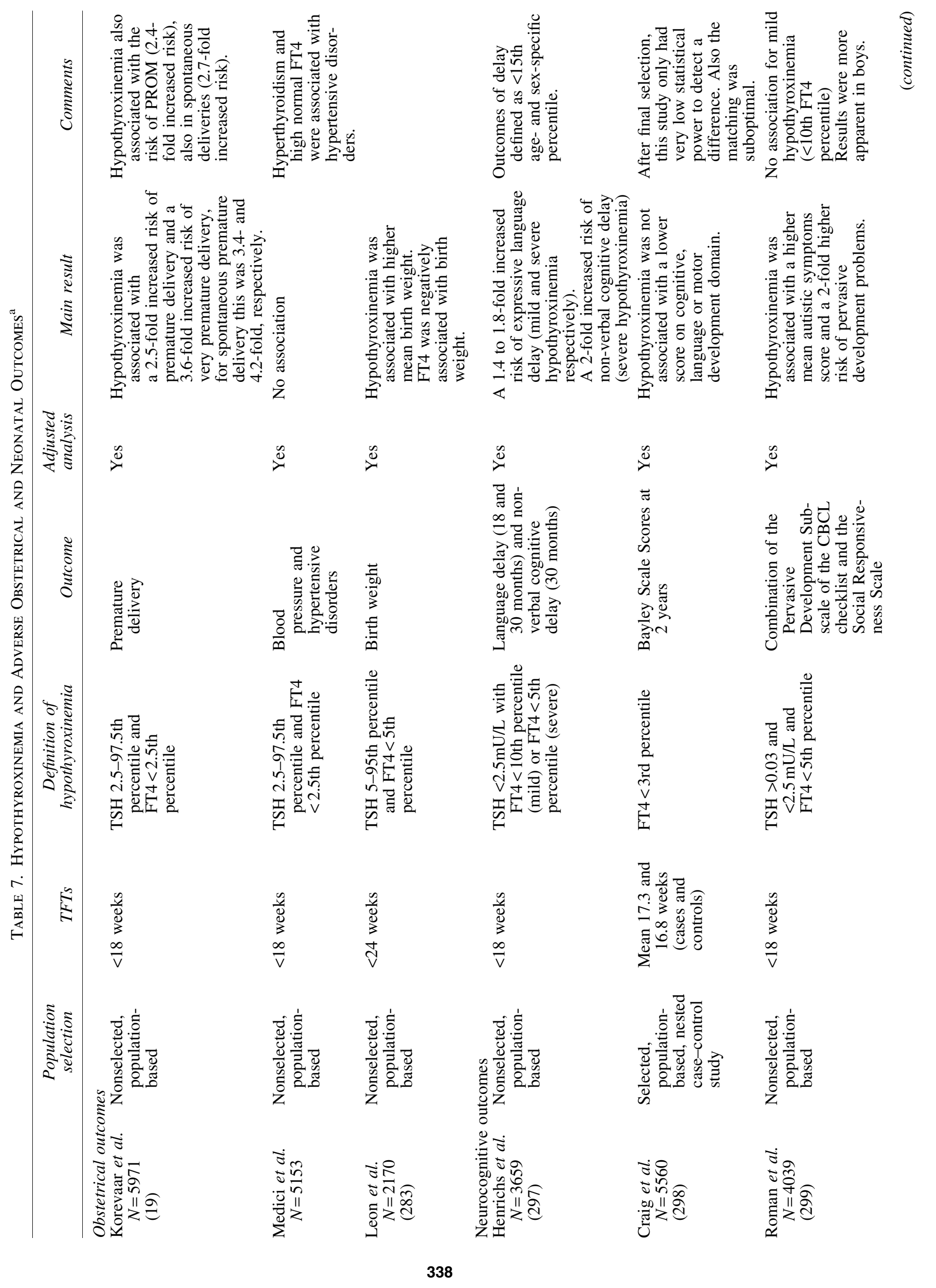




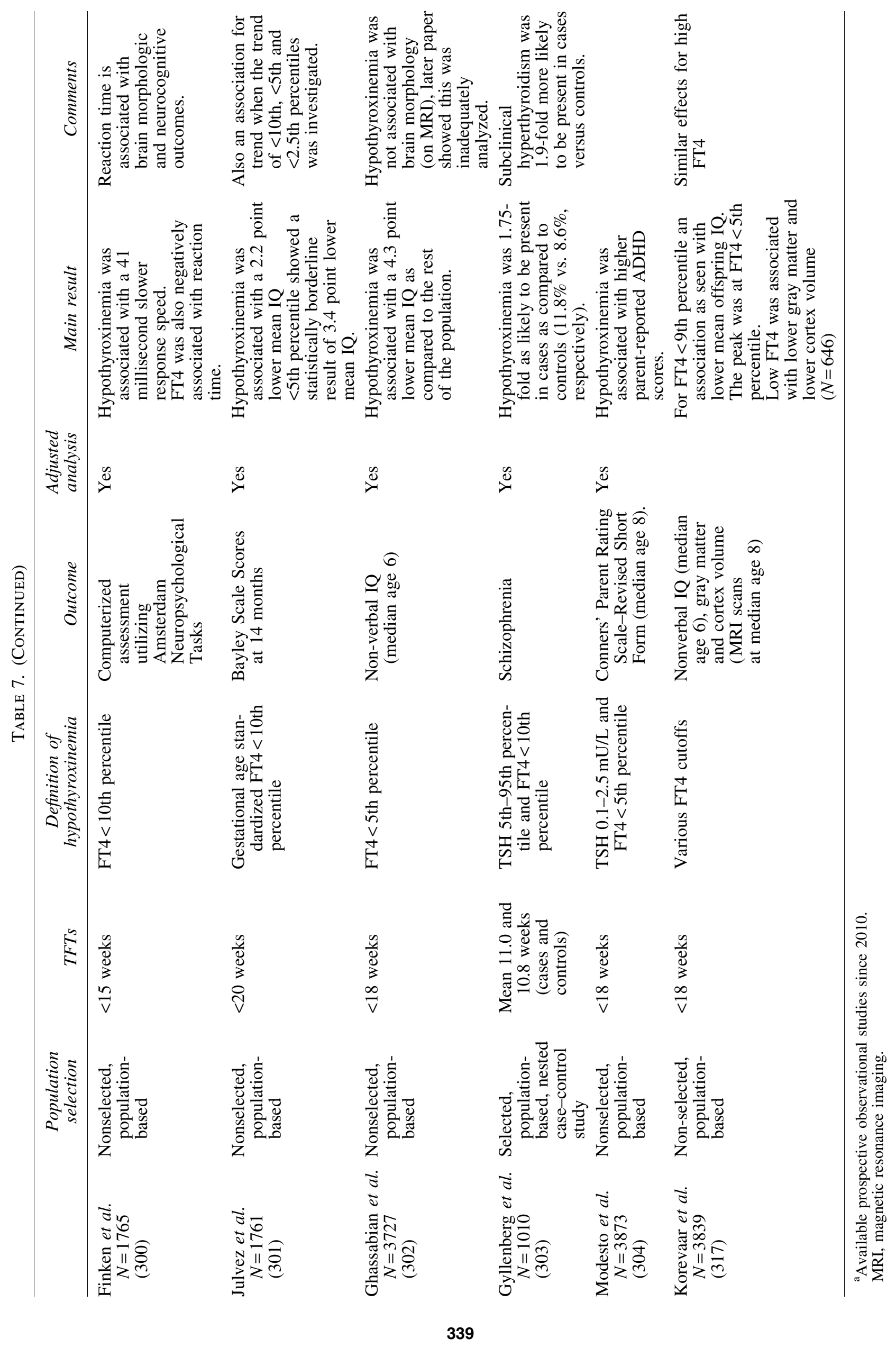


- RECOMMENDATION 27

Treatment of overt hypothyroidism is recommended during pregnancy.

Strong recommendation, moderate-quality evidence.

\section{Question 37: Should women with subclinical hypothyroidism be treated in pregnancy?}

Many prospective and retrospective studies have demonstrated an increased risk of pregnancy complications associated with mildly elevated maternal TSH concentrations, especially in TPOAb-positive women. However, only a small number of studies have investigated the impact of LT4 treatment on pregnancy complications in such women.

As noted above, a single RCT has demonstrated a potential benefit of LT4 intervention at $\sim 9$ weeks gestation. Importantly, this study documented a reduction in the adverse pregnancy composite outcome only in TPOAb-positive women with mild hypothyroidism (defined as a TSH $>2.5 \mathrm{mU} / \mathrm{L}$ ) (286). It should again be noted that the majority of women with subclinical hypothyroidism detected in this investigation were TPOAb negative, for whom no intervention or treatment was provided. This study also used a composite endpoint that included subjective endpoints such as cesarean section rates and postdelivery admission to the neonatal intensive care unit. Importantly, the authors' conclusion for their primary endpoint stated that universal screening for elevated TSH concentration in a broad population of pregnant women did not improve outcomes compared to a high risk screening strategy. Outcomes of universal screening compared to no screening were not assessed.

A separate RCT demonstrated a decrease in preterm delivery and pregnancy loss in euthyroid (defined as TSH $<4.2 \mathrm{mU} / \mathrm{L}$ ) TPOAb-positive women who were treated with LT4 beginning in the first trimester of pregnancy (28). However, the majority of pregnancy losses in the control group occurred before the average start of LT4 therapy.

Many studies have stratified the risk imparted by hypothyroidism according to TPOAb status and consistently show that this risk is higher in TPOAb-positive women (288). These data also suggest that the adverse impact associated with maternal TSH levels is apparent at lower TSH elevations in women known to be TPOAb positive compared to women who are TPOAb negative. Furthermore, two studies suggest a reduction in pregnancy loss when TPOAb-positive women are treated with LT4, even when biochemically euthyroid $(28,180)$. Intervention trials have not been performed in TPOAb-negative women.

However, despite the limitations of available interventional trials of LT4 therapy in this subclinically hypothyroid group, the data taken in aggregate appear to suggest a benefit of treatment, especially as it applies to reducing miscarriage in TPOAb-positive women. Therefore, it seems reasonable to recommend or consider LT4 treatment for specific subgroups of pregnant women with subclinical hypothyroidism. The strength of such recommendations, however, should differ depending on TPOAb status, as will the strength of evidence supporting treatment for each subgroup. This recommendation also necessitates that any pregnant women with an elevated TSH concentration must also be evaluated for TPOAb status. In making the recommendation, the task force acknowledges the very low risk inherent in initiating low-dose
LT4 treatment. A dose of only $50 \mu \mathrm{g} / \mathrm{d}$ is typically required for effective treatment of subclinically hypothyroid women.

\section{- RECOMMENDATION 28}

Pregnant women with TSH concentrations $>2.5 \mathrm{mU} / \mathrm{L}$ should be evaluated for TPOAb status.

\section{- RECOMMENDATION 29}

Subclinical hypothyroidism in pregnancy should be approached as follows:

(a) LT4 therapy is recommended for

- TPOAb-positive women with a TSH greater than the pregnancy-specific reference range (see Recommendation 1).

Strong recommendation, moderate-quality evidence.

- TPOAb-negative women with a TSH greater than $10.0 \mathrm{mU} / \mathrm{L}$.

Strong recommendation, low-quality evidence.

(b) LT4 therapy may be considered for

- TPOAb-positive women with TSH concentrations $>2.5 \mathrm{mU} / \mathrm{L}$ and below the upper limit of the pregnancy-specific reference range.

Weak recommendation, moderate-quality evidence.

- TPOAb-negative women and TPOAb-negative women with TSH concentrations greater than the pregnancyspecific reference range and below $10.0 \mathrm{mU} / \mathrm{L}$.

Weak recommendation, low-quality evidence.

(c) LT4 therapy is not recommended for

- TPOAb-negative women with a normal TSH (TSH within the pregnancy-specific reference range or $<4.0 \mathrm{mU} / \mathrm{L}$ if unavailable).

Strong recommendation, high-quality evidence.

\section{Question 38: Should women with isolated hypothyroxinemia be treated with LT4 in pregnancy?}

Although several studies have reported adverse outcomes in children born to mothers with isolated hypothyroxinemia, no interventional data have yet been published that demonstrate beneficial effects of LT4 therapy. One observational study analyzing women at 12, 24, and 32 weeks of pregnancy demonstrated delayed infant neurodevelopment in women with persistent hypothyroxinemia. However, when FT4 concentrations increased during pregnancy, infant development was not improved (312). Nevertheless, at present there are only two randomized, prospective, intervention trials in which women with a low FT4 were treated with LT4, at 13 and 17 weeks gestation, respectively $(21,306)$. Both investigations failed to show any beneficial effect on cognitive development following LT4 administration, though a major limitation of the studies was the late timing of the intervention, after completion of the first trimester. Nevertheless, given the existing interventional data, treatment of isolated hypothyroxinemia cannot be recommended at this time.

\section{- RECOMMENDATION 30}

Isolated hypothyroxinemia should not be routinely treated in pregnancy.

Weak recommendation, low-quality evidence. 


\section{Question 39: What is the optimal method of treating hypothyroidism in pregnant women?}

Delivery of T4 is crucial for the developing fetal brain (313). The ratio of T4 to T3 in desiccated thyroid preparations is $4.2: 1$, which is significantly lower than the $14: 1$ ratio of secretion by the human thyroid gland. This relative excess of T3 leads to supraphysiologic maternal levels of T3 and relatively low levels of T4 (319). Patients using either desiccated thyroid or a treatment regimen combining T3 and T4 (320) are likely at risk for having insufficient transfer of maternal T4 to the fetal brain. It is notable that the majority of fetal T3 present in the CNS during pregnancy is derived from maternal T4 actively transported into this space. The fetal CNS is relatively impermeable to T3, which therefore argues against use of exogenous T3 during pregnancy. For these reasons, the task force feels that any T3-containing preparation should be avoided for the treatment of maternal hypothyroidism during pregnancy.

\section{- RECOMMENDATION 31}

The recommended treatment of maternal hypothyroidism is administration of oral LT4. Other thyroid preparations such as T3 or desiccated thyroid should not be used in pregnancy.

Strong recommendation, low-quality evidence.

\section{Question 40: What is the biochemical goal when treating hypothyroidism in pregnant women?}

\section{- RECOMMENDATION 32}

In parallel to the treatment of hypothyroidism in a general population, it is reasonable to target a TSH in the lower half of the trimester-specific reference range. When this is not available, it is reasonable to target maternal TSH concentrations below $2.5 \mathrm{mU} / \mathrm{L}$.

Weak recommendation, moderate-quality evidence.

\section{Question 41: Are there circumstances in which euthyroid women are at risk for hypothyroidism once pregnant?}

In 1994, Glinoer et al. (147) performed a prospective study in 87 TPOAb-positive euthyroid women evaluated before and during early pregnancy. Twenty percent of women in the study developed a TSH $>4 \mathrm{mU} / \mathrm{L}$ during gestation despite a normal TSH and no requirement for LT4 prenatally. This occurred despite the expected decrease in TPOAb titers during pregnancy (147). Negro et al. (28) demonstrated similar results in a prospective study. The authors found that in TPOAb-positive euthyroid women, TSH levels increased progressively as gestation progressed, from a mean of $1.7 \mathrm{mU} / \mathrm{L}$ (12th week) to $3.5 \mathrm{mU} / \mathrm{L}$ (term), with $19 \%$ of women having a supranormal TSH concentration at delivery. These findings confirm that an increased requirement for thyroid hormone occurs during gestation. In women who are TPOAb positive, both overt and subclinical hypothyroidism may occur because of a lack of ability of the thyroid to augment production when needed during pregnancy. Similarly, patients who undergo hemithyroidectomy or receive radioactive iodine and are euthyroid before preg- nancy are at risk for developing elevated serum TSH levels during gestation.

In summary, euthyroid patients who are antithyroid $\mathrm{Ab}$ positive, post-hemithyroidectomy, or treated with radioactive iodine have an increased propensity for the development of hypothyroidism in gestation and should be monitored regularly.

\section{Question 42: How should women with hypothyroidism or at risk for hypothyroidism be monitored through pregnancy?}

In women at risk for hypothyroidism (TPOAb- or TgAbpositive, post-hemithyroidectomy, and/or post-radioactive iodine) increased surveillance is recommended. Based on findings extrapolated from investigations of treated hypothyroid women from early pregnancy onwards (321), it is reasonable to evaluate these women for TSH elevation approximately every 4 weeks during pregnancy. Serial testing is preferably continued through midpregnancy because the increased T4 demand continues throughout the first half of gestation.

A study by Yassa and colleagues (322) investigated the optimal timing of subsequent assessment of thyroid function following dose modification though this was in patients consuming LT4 prenatally and may not be generalizable to patients not taking LT4 but at risk for hypothyroidism.

\section{- RECOMMENDATION 33}

Women with overt and subclinical hypothyroidism (treated or untreated) or those at risk for hypothyroidism (e.g., patients who are euthyroid but TPOAb or TgAb positive, post-hemithyroidectomy, or treated with radioactive iodine) should be monitored with a serum TSH measurement approximately every 4 weeks until midgestation and at least once near 30 weeks gestation.

\section{Strong recommendation, high-quality evidence.}

\section{Question 43: How do treated hypothyroid women (receiving LT4) differ from other patients during pregnancy? What changes can be anticipated in such patients during gestation?}

The major physiologic thyroid changes during pregnancy have been thoroughly studied. Total body T4 requirements are not static throughout gestation. Rather, data demonstrate that the total body T4 pool must increase by approximately $40 \%-50 \%$ to maintain a euthyroid state $(321,323)$. In a healthy woman who becomes pregnant, the pregnancy hormone hCG plays a major role as a stimulus of maternal thyroid hormone production, especially throughout the first half of pregnancy. Together with pituitary TSH, placental hCG stimulates endogenous thyroid hormone production when an intact thyroid is present, and it helps to maintain a euthyroid state during gestation.

In women with known hypothyroidism, serum hCG and $\mathrm{TSH}$ cannot stimulate adequate $\mathrm{T} 4$ production. If exogenous LT4 is not adjusted, the increased demand of pregnancy will outstrip supply, and maternal hypothyroidism will occur. Clinical studies have confirmed that the increased requirement for thyroxine (or exogenous LT4) occurs as early as 4-6 weeks of pregnancy (321). Such requirements gradually 
increase through 16-20 weeks of pregnancy and plateau thereafter until the time of delivery. These data provide the basis for recommending adjustments of LT4 dosage when affected women become pregnant and also for the timing of follow-up intervals for TSH in treated patients.

\section{Question 44: What proportion of treated hypothyroid women (receiving LT4 prenatally) require changes in their $L T 4$ dose during pregnancy?}

Between $50 \%$ and $85 \%$ of LT4-treated hypothyroid women need to increase exogenous LT4 dosing during pregnancy $(277,321,323)$. The incremental increase largely depends on the underlying etiology of the hypothyroidism. There is a greater likelihood that dose increases will be required in those patients without functional thyroid tissue (e.g., due to radioablation or surgery) in comparison with patients with Hashimoto's thyroiditis $(324,325)$. The preconception level of TSH as well as other factors can also influence the rapidity and extent of LT4 augmentation necessary to maintain a euthyroid state during pregnancy. For example, variation and changes in maternal estrogen levels during pregnancy correlate with variations in the gestational requirements for LT4 (321).

The LT4 adjustment should be made as soon as possible after pregnancy is confirmed to reduce the probability of hypothyroidism. Normalization of TSH concentrations throughout gestation is the goal. For women receiving LT4 preconception, a prospective, randomized study has provided evidence that supports a single dose-adjustment strategy rather than a stepwise approach for LT4 dosage adjustment postconception (322). For euthyroid women receiving oncedaily dosing of LT4 (regardless of the dosage), a recommendation to increase by two additional tablets weekly (nine tablets per week instead of seven tablets per week, giving a $29 \%$ increase) can effectively mimic gestational physiology and thus prevent maternal hypothyroidism during the first trimester (322). Another option is to increase the dosage of daily LT4 by approximately 25\%-30\%. Dosage augmentation should occur as soon as possible when a missed menstruation or suspected pregnancy occurs, and this should be discussed with every patient in the prepregnancy setting. Confirmatory biochemical testing should also occur simultaneously.

\section{Question 45: How should preconception LT4 be adjusted in treated hypothyroid women (receiving LT4) planning pregnancy?}

The difficulties inherent to achieving rapid, postconceptional TSH normalization have also focused attention upon preconception TSH modulation. Different cutoff values for preconception TSH, ranging from $<1.2$ to $<2.5 \mathrm{mU} / \mathrm{L}$ have been advocated. In one study, only $17 \%$ of women with TSH $<1.2 \mathrm{mU} / \mathrm{L}$ had to increase LT4 dose later during pregnancy (326). Given this, it is recommended that all treated hypothyroid women (currently receiving LT4) optimize thyroid parameters preconception. A maternal serum TSH concentration $<2.5 \mathrm{mU} / \mathrm{L}$ is a reasonable goal for such women. Even lower preconception TSH values $(<1.5 \mathrm{mU} / \mathrm{L})$ could reduce the risk of TSH elevation during the first trimester (322), but a lower treatment target may not improve outcomes because the LT4 dose can be immediately increased upon a positive pregnancy test. Furthermore, the process of achieving a TSH concentration at the lower end of the reference range could induce subnormal TSH concentrations in some patients. Though generally safe for any developing fetus, potential such effects upon conception and/or successful implantation are unknown.

\section{- RECOMMENDATION 34}

Treated hypothyroid women of reproductive age should be counseled regarding the likelihood of increased demand for LT4 during pregnancy. Such women should also be counseled to contact their caregiver immediately upon a confirmed or suspected pregnancy.

\section{Strong recommendation, high-quality evidence.}

\section{- RECOMMENDATION 35}

In hypothyroid women treated with LT4 who are planning pregnancy, serum TSH should be evaluated preconception, and LT4 dose adjusted to achieve a TSH value between the lower reference limit and $2.5 \mathrm{mU} / \mathrm{L}$.

Strong recommendation, moderate-quality evidence.

\section{- RECOMMENDATION 36}

Hypothyroid patients receiving LT4 treatment with a suspected or confirmed pregnancy (e.g., positive home pregnancy test) should independently increase their dose of LT4 by $\sim 20 \%-30 \%$ and urgently notify their caregiver for prompt testing and further evaluation. One means of accomplishing this is to administer two additional tablets weekly of the patient's current daily LT4 dosage.

\section{Strong recommendation, high-quality evidence.}

\section{Question 46: How should LT4 be adjusted postpartum?}

The increased LT4 dose requirements during gestation are a function of pregnancy itself. Therefore, following delivery, maternal LT4 dosing should be reduced to prepregnancy levels, and a serum TSH assessed 6 weeks thereafter. However, a study demonstrated that more than $50 \%$ of women with Hashimoto's thyroiditis required an increase in the pregestational thyroid hormone dose in the postpartum period, presumably due to an exacerbation of autoimmune thyroid dysfunction post partum (327). In women started on LT4 during pregnancy for thyroid autoimmunity in the absence of TSH elevation, the LT4 can be stopped at delivery, with serum TSH assessment at 6 weeks post partum.

\section{- RECOMMENDATION 37}

Following delivery, LT4 should be reduced to the patient's preconception dose. Additional thyroid function testing should be performed at approximately 6 weeks post partum.

Strong recommendation, moderate-quality evidence.

\section{- RECOMMENDATION 38}

Some women in whom LT4 is initiated during pregnancy may not require LT4 post partum. Such women are candidates for discontinuing LT4, especially when the LT4 
dose is $\leq 50 \mu \mathrm{g} / \mathrm{d}$. The decision to discontinue LT4, if desired, should be made by the patient and their caregiver. If LT4 is discontinued, serum TSH should be evaluated in approximately 6 weeks.

Weak recommendation, moderate-quality evidence.

\section{Question 47: What is the outcome and long-term prognosis when maternal hypothyroidism is effectively treated through gestation?}

Although untreated (or incompletely treated) hypothyroidism can adversely affect pregnancy, no data suggest that women with adequately treated subclinical or overt hypothyroidism have an increased risk of any obstetrical complication. Consequently, there is no indication for any additional obstetric testing or surveillance in pregnancies of women with either subclinical or overt hypothyroidism who are being monitored and treated appropriately.

\section{Question 48: Except for measurement of maternal thyroid function, should additional maternal or fetal testing occur in treated hypothyroid women during pregnancy?}

\section{- RECOMMENDATION 39}

In the care of women with adequately treated hypothyroidism, no other maternal or fetal testing (such as serial fetal ultrasounds, antenatal testing, and/or umbilical blood sampling) is recommended beyond measurement of maternal thyroid function unless needed due to other circumstances of pregnancy. An exception to this is women with GD effectively treated with ${ }^{131}$ I ablation or surgical resection, who require TSH receptor antibody (TRAb) monitoring.

Strong recommendation, moderate-quality evidence.

\section{THYROTOXICOSIS IN PREGNANCY}

\section{Question 49: What are the causes of thyrotoxicosis in pregnancy?}

Thyrotoxicosis is the clinical syndrome of hypermetabolism and hyperactivity that results when a person is exposed to supraphysiological amounts of thyroid hormones. The most common cause of thyrotoxicosis is hyperfunction of the thyroid gland (hyperthyroidism), and the most common cause of hyperthyroidism in women of childbearing age is autoimmune GD occurring before pregnancy in $0.4 \%-1.0 \%$ of women and in approximately $0.2 \%$ during pregnancy (328).

Less common non-autoimmune causes of hyperthyroidism in pregnancy include toxic multinodular goiter and toxic adenoma. Subacute painful or painless thyroiditis with passive release of thyroid hormones from a damaged thyroid gland are less common causes of thyrotoxicosis in pregnancy, and a number of other conditions such as a TSH-secreting pituitary adenoma (329), struma ovarii (330), functional thyroid cancer metastases, or germline TSH receptor mutations (331) are very rare. A special cause of thyrotoxicosis is overtreatment with or factitious intake of thyroid hormone.

More frequent than GD as the cause of thyroid function tests demonstrating hyperthyroxinemia is "gestational transient thyrotoxicosis," which is limited to the first half of pregnancy. This condition, characterized by elevated FT4 and suppressed serum TSH, is diagnosed in about 1\%-3\% of pregnancies. This frequency depends on the geographic area and is secondary to elevated hCG levels $(332,333)$. Often it is associated with hyperemesis gravidarum, defined as severe nausea and vomiting in early pregnancy with more than 5\% weight loss, dehydration, and ketonuria. Hyperemesis gravidarum occurs in 3-10 per 1000 pregnancies $(334,335)$. Other conditions associated with hCG-induced thyrotoxicosis include multiple gestation, hydatidiform mole, and choriocarcinoma (336,337). Most cases present with marked elevations of serum hCG (14). A TSH receptor mutation leading to functional hypersensitivity to $\mathrm{hCG}$ also has been recognized as a rare cause of pregnancy-associated hyperthyroidism (338).

\section{Question 50: What is the appropriate initial evaluation of a suppressed serum TSH concentration during the first trimester of pregnancy?}

Serum TSH may decrease in the first trimester of normal pregnancy as a physiological response to the stimulating effect of hCG upon the TSH receptor. A peak hCG level typically occurs between 7 and 11 weeks gestation (339). In particular, a serum TSH below $0.1 \mathrm{mU} / \mathrm{L}$ (in some cases even undetectable) may be present in approximately $5 \%$ of women by week 11 of pregnancy (270). Any subnormal serum TSH value should be evaluated in conjunction with serum TT4 (or FT4) and T3 values. The biochemical diagnosis of overt hyperthyroidism is confirmed in the presence of a suppressed or undetectable serum TSH and inappropriately elevated serum TT4/FT4, or T3 (Section III).

\section{Question 51: How can gestational transient thyrotoxicosis be differentiated from Graves' hyperthyroidism in pregnancy?}

Diagnosing the cause of the disease is essential in any patient with thyrotoxicosis. In early pregnancy, the differential diagnosis in the majority of cases is between Graves' hyperthyroidism and gestational transient thyrotoxicosis $(332,333)$. In both situations, common clinical manifestations include palpitations, anxiety, tremor, and heat intolerance. A careful history and physical examination is of utmost importance in establishing the etiology. The findings of no prior history of thyroid disease, no stigmata of GD (goiter, orbitopathy), a self-limited mild disorder, and symptoms of emesis favor the diagnosis of gestational transient thyrotoxicosis.

If other causes for thyrotoxicosis are suspected, measurement of TRAb is indicated. If this is negative or thyroid nodules are suspected based on clinical examination, a thyroid ultrasound should be performed to evaluate nodularity. Serum hCG is higher on average in gestational transient thyrotoxicosis than in patients with GD, but overlap is considerable and the clinical usefulness of such measurement is limited (340). No study has demonstrated usefulness of thyroid ultrasonography for differentiating between gestational transient thyrotoxicosis and GD. In the presence of a nodular goiter, a serum TT3 determination is helpful in assessing the possibility of the "T3 toxicosis" syndrome. TT3 determination may also be of benefit in diagnosing $\mathrm{T} 3$ thyrotoxicosis 
caused by GD. In general, serum T3 tends to be disproportionately elevated more than $\mathrm{T} 4$ in cases of thyrotoxicosis caused by direct thyroid hyperactivity. In comparison, T4 tends to be disproportionately elevated beyond T3 when thyrotoxicosis is caused by destructive processes such as thyroiditis (341).

\section{- RECOMMENDATION $\mathbf{4 0}$}

When a suppressed serum TSH is detected in the first trimester (TSH less than the reference range), a medical history, physical examination, and measurement of maternal serum FT4 or TT4 concentrations should be performed. Measurement of TRAb and maternal TT3 may prove helpful in clarifying the etiology of thyrotoxicosis.

Strong recommendation, moderate-quality evidence.

\section{- RECOMMENDATION 41}

Radionuclide scintigraphy or radioiodine uptake determination should not be performed in pregnancy.

Strong recommendation, high-quality evidence.

\section{Question 52: What is the appropriate management of gestational transient thyrotoxicosis?}

The management of women with gestational transient thyrotoxicosis depends on the severity of symptoms. In women with hyperemesis gravidarum, control of vomiting and treatment of dehydration with intravenous fluids is the customary treatment. Women with severe hyperemesis gravidarum need frequent medical visits for management of dehydration and electrolyte abnormalities. In some cases, hospitalization is required. Antithyroid drugs (ATDs) are not indicated because the serum T4 returns to normal by 14-18 weeks gestation and ATD use in early pregnancy increases risk of birth defects. Importantly, obstetrical outcome was not improved in isolated cases in which gestational transient thyrotoxicosis was treated with ATDs (342). However, there are no studies reported in the literature comparing ATD therapy versus supportive therapy. In situations in which symptomatic therapy is indicated, small amounts of $\beta$ blockers given over a limited time period may be useful, and close follow-up with repeat investigation for the cause of disease should be performed.

\section{- RECOMMENDATION 42}

The appropriate management of abnormal maternal thyroid tests attributable to gestational transient thyrotoxicosis and/or hyperemesis gravidarum includes supportive therapy, management of dehydration, and hospitalization if needed. ATDs are not recommended, though $\beta$-blockers may be considered.

\section{Strong recommendation, moderate-quality evidence.}

\section{Question 53: How should women with GD seeking future pregnancy be counseled?}

The planning of therapy in relation to possible future pregnancy should be discussed with all women of childbearing age who develop thyrotoxicosis. In general, pregnancy should be postponed until a stable euthyroid state is reached. As a guide, two sets of thyroid function test within the reference range, at least 1 month apart, and with no change in therapy between tests, can be used to define a stable euthyroid state. The use of contraception until the disease is controlled is strongly recommended.

A hyperthyroid patient who desires future pregnancy may be offered ablative therapy using ${ }^{131} \mathrm{I}$, thyroid surgery, or medical therapy. Each therapeutic option carries advantages and disadvantages, as detailed below (see Table 8). This is further discussed in the ATA guidelines for the management of thyrotoxicosis (343).

Table 8. Advantages and Disadvantages of Therapeutic Options for Women with Graves' Disease Seeking Future Pregnancy

\begin{tabular}{|c|c|c|}
\hline Therapy & Advantages & Disadvantages \\
\hline \multirow[t]{3}{*}{$\begin{array}{l}\text { Antithyroid } \\
\text { drugs }\end{array}$} & $\begin{array}{l}\text { Effective treatment to euthyroid state within } \\
1-2 \text { months }\end{array}$ & $\begin{array}{l}\text { Medication adverse effects (mild 5\%-8\%; severe } \\
0.2 \% \text { ) }\end{array}$ \\
\hline & $\begin{array}{l}\text { Often induces gradual remission of } \\
\text { autoimmunity (decreasing antibody titers) }\end{array}$ & $\begin{array}{l}\text { Birth defects associated with use during preg- } \\
\text { nancy (MMI 3\%-4\%; PTU 2\%-3\% though } \\
\text { less severe) }\end{array}$ \\
\hline & $\begin{array}{l}\text { Easily discontinued or modified. Treatment } \\
\text { easy to take. Relatively inexpensive }\end{array}$ & Relapse after drug withdrawal likely in $50 \%-70 \%$ \\
\hline \multirow{3}{*}{$\begin{array}{l}\text { Radioactive } \\
\text { iodine }\end{array}$} & Easy oral administration & Repeat therapy at times necessary \\
\hline & Reduction in goiter size & $\begin{array}{l}\text { Rising antibody titers following treatment may } \\
\text { contribute to worsening orbitopathy or fetal } \\
\text { risk }\end{array}$ \\
\hline & Future relapse of hyperthyroidism very rare & $\begin{array}{l}\text { Lifelong need of levothyroxine therapy } \\
\text { following ablation }\end{array}$ \\
\hline \multirow[t]{3}{*}{ Thyroidectomy } & $\begin{array}{l}\text { Definitive therapy of hyperthyroidism. Stable } \\
\text { euthyroid state easily achieved on re- } \\
\text { placement levothyroxine therapy }\end{array}$ & $\begin{array}{l}\text { Life-long need for levothyroxine } \\
\text { supplementation }\end{array}$ \\
\hline & $\begin{array}{l}\text { Post surgery, gradual remission of autoim- } \\
\text { munity occurs }\end{array}$ & Surgical complications occur in $2 \%-5 \%$ \\
\hline & Goiter disappears & $\begin{array}{l}\text { Healing and recovery from surgery } \\
\text { Permanent neck scar }\end{array}$ \\
\hline
\end{tabular}


Ablative therapy. If the patient opts for radioactive iodine ablative therapy prior to pregnancy, the following recommendations should be provided. First, TRAb levels tend to increase following ${ }^{131}$ I therapy and may remain elevated for many months following ${ }^{131} \mathrm{I}$ therapy. Therefore, patients with high TRAb levels or severe hyperthyroidism may favor consideration of other therapeutic options such as surgery (344). Second, a subset of young patients with severe GD may not become stably euthyroid within the first year after ${ }^{131} \mathrm{I}$ therapy $(345,346,347,348)$. Third, if ${ }^{131} \mathrm{I}$ therapy is planned, a pregnancy test should be performed 48 hours before ${ }^{131} \mathrm{I}$ ablation to confirm absence of unexpected pregnancy. Fourth, conception should be delayed 6 months and until a stable euthyroid state is reached after ablation and initiation of LT4 replacement therapy.

Antithyroid drugs. If the patient chooses ATD therapy, the following recommendations should be given. First, the increased risk of birth defects associated with both PTU and MMI use during early pregnancy should be reviewed, Second, if possible, ATDs should be avoided in the first trimester of pregnancy, but when necessary PTU is generally favored. Third, consideration can be given to discontinuing PTU after the first trimester and switching to MMI to decrease the risk of liver failure in the mother.

\section{- RECOMMENDATION 43}

In all women of childbearing age who are thyrotoxic, the possibility of future pregnancy should be discussed. Women with GD seeking future pregnancy should be counseled regarding the complexity of disease management during future gestation, including the association of birth defects with ATD use. Preconception counseling should review the risks and benefits of all treatment options and the patient's desired timeline to conception.

\section{Strong recommendation, high-quality evidence.}

\section{- RECOMMENDATION 44}

Thyrotoxic women should be rendered stably euthyroid before attempting pregnancy. Several treatment options exist, each of which are associated with risks and benefits. These include ${ }^{131} \mathrm{I}$ ablation, surgical thyroidectomy, or ATD therapy.

Strong recommendation, moderate-quality evidence.

\section{Question 54: What is the management of patients with Graves' hyperthyroidism during pregnancy?}

Several studies have shown that obstetric and medical complications are directly related to control of maternal hyperthyroidism, and the duration of the euthyroid state throughout pregnancy $(339,342,344,349)$. Poor control of thyrotoxicosis is associated with pregnancy loss, pregnancyinduced hypertension, prematurity, low birth weight, intrauterine growth restriction, stillbirth, thyroid storm, and maternal congestive heart failure (350). Moreover, some studies suggest fetal exposure to excessive levels of maternal thyroid hormone may program the offspring to develop diseases such as seizure disorders and neurobehavioral disorders in later life (351).
Thionamide ATDs (MMI, carbimazole [CM], and PTU) are the mainstays of treatment for hyperthyroidism during pregnancy $(352,353)$. They reduce iodine organification and coupling of monoiodotyrosine and diiodotyrosine, therefore inhibiting thyroid hormone synthesis. Because the block is not absolute and the thyroid contains a depot of thyroid hormone bound to $\mathrm{Tg}$, the normalization of thyroid function tests takes place gradually over weeks. The initial dose of ATD depends on the severity of the symptoms and the degree of hyperthyroxinemia. In general, initial doses of ATDs during pregnancy are as follows: MMI, 5-30 mg/d (typical dose in average patient 10-20 mg); CM, 10-40 mg/d; and PTU, 100-600 mg/d (typical PTU dose in average patient $200-400 \mathrm{mg} / \mathrm{d}$ ). The equivalent potency of MMI to PTU is approximately 1:20 (e.g., $5 \mathrm{mg}$ MMI = $100 \mathrm{mg}$ of PTU) (354-356). Ten milligrams of CM is rapidly metabolized to approximately $6 \mathrm{mg}$ of MMI (328). Because the half-life of PTU is shorter than that of MMI, PTU dosing should generally be split into two or three daily doses. In comparison, MMI can generally be given in one daily dose. In rare cases of severe hyperthyroidism, twice or threetimes daily dosing may be of benefit $(357,358)$.

Importantly, side effects occur in $3 \%-5 \%$ of patients taking thionamide drugs, the majority of which are allergic reactions such as skin rash (352), whereas the severe side effects of agranulocytosis $(0.15 \%)(359,360)$ and liver failure $(<0.1 \%)(92)$ are rare. Most side effects develop within the first months following initiation (359) or re-initiation (361) of therapy. In 2010 the U.S. Food and Drug Administration (FDA) called attention to the risk of hepatotoxicity in patients exposed to PTU because PTU had been found to be third on the list of drugs leading to liver transplantation in the United States $(362,363)$. An advisory committee recommended limiting the use of PTU to the first trimester of pregnancy (364). Exceptions to this are patients with MMI allergy or those with thyroid storm. Monitoring hepatic enzymes during administration of PTU may be considered. However, no prospective data exist that have demonstrated that the monitoring of liver enzymes is effective in preventing fulminant PTU-induced hepatotoxicity.

However, the greatest risk surrounding the use of ATDs in pregnancy is related to their potential teratogenic effects (365). As early as 1972, exposure to MMI had been associated with aplasia cutis (366). Subsequently, several other types of congenital malformations have been associated with MMI use. A "syndrome of methimazole/carbimazole embryopathy" was described, which also includes dysmorphic facies $(367,368)$. Apart from aplasia cutis, defects with a statistically significant association with the use of MMI include choanal or esophageal atresia; various types of abdominal wall defects including umbilicocele; and eye, urinary system, and ventricular septal defects $(369,370,371)$. Recent studies have shown that these complications are more common than previously thought, and they affect $2 \%-4 \%$ of children who have been exposed to MMI in early pregnancy, especially during gestational weeks 6-10 (369,370,372).

PTU was previously considered a safe medication for use during gestation $(364,367)$. Recently, however, a Danish study revealed that $2 \%-3 \%$ of children exposed to PTU developed birth defects associated with this therapy (370). The defects were primarily face and neck cysts (often considered to be minor birth defects) and urinary tract abnormalities (in males). Prior to the recent investigation, such abnormalities 
were not commonly associated with PTU exposure, likely because they were diagnosed later in life when complications ensued. Importantly, however, most affected patients received surgery for the abnormality (373). Thus, PTU-associated birth defects appear less severe than MMI-associated birth defects but occur with similar incidence.

Beta-adrenergic blocking agents, such as propranolol 10 $40 \mathrm{mg}$ every 6-8 hours may be used for controlling hypermetabolic symptoms until patients have become euthyroid on ATD therapy. The dose should be reduced as clinically indicated. In the vast majority of cases the drug can be discontinued in 2-6 weeks. Long-term treatment with $\beta$-blockers has been associated with intrauterine growth restriction, fetal bradycardia, and neonatal hypoglycemia (374). One study suggested a higher rate of spontaneous pregnancy loss when both drugs were taken together, as compared with patients receiving only MMI (375). However, it was not clear that this difference was attributable to the medication as opposed to the underlying condition. Beta-blocking drugs may be used as preparation for thyroidectomy.

\section{Question 55: Should antithyroid medication be withdrawn or modified in early pregnancy?}

Many patients receiving ATD therapy for GD gradually enter remission of the autoimmune abnormality when made euthyroid. Though approximately half of patients eventually experience a relapse of hyperthyroidism when the antithyroid medication is withdrawn after 1-2 years of therapy, only a small fraction of patients who have become TRAb negative during therapy will become hyperthyroid within the first months. In support of this, in a prospective Norwegian study of 218 GD patients treated with ATD for 12 months, only 5\% of TRAb-negative patients became hyperthyroid within 8 weeks after ATD withdrawal (376).

Thus, one option when pregnancy is diagnosed in a woman receiving ATD therapy for GD and who, based on clinical and biochemical findings appears to be in remission, is to withdraw ATD medication and perform repeated thyroid function testing during the first trimester of pregnancy. If ATD therapy is needed during the first trimester, PTU is preferred over MMI because the risk for severe birth defects is lower. Cessation of medication has to be recommended early in gestation, before the major teratogenic periods (gestational weeks 6-10) $(372,373,376,377)$.

Gestational weeks are calculated from the first day of the last normal menstrual period; it is typically at week 5 when the next normal menstruation does not appear in a pregnant woman. Sensitive pregnancy tests are widely available and should detect pregnancy by this time. Optimally, women receiving ATD should test for pregnancy within the first days of missing or unusually weak menstruation. If the test is positive, the woman should contact the caregiver responsible for thyroid therapy to receive instruction regarding withdrawing or modifying ATD therapy and to discuss thyroid function testing.

The risk of rapid relapse of hyperthyroidism after medication withdrawal in early pregnancy varies among patients. The risk is high in patients who have been treated for a short period ( $<6$ months), who have suppressed or low serum TSH while on medication prepregnancy, who require $>5-10 \mathrm{mg}$ of MMI per day to stay euthyroid, who have active orbitopathy or large goiter, and those who have high levels of TRAb (378). Because this assessment is based upon clinical judgment, no specific single parameter may be used to assign risk. However, if the risk is considered high, medication should not be withdrawn, and PTU should be administered as the drug of choice.

Even if conventional ATDs are effective in achieving a euthyroid state, the risk of birth defects has raised the question of alternative types of drug therapy for hyperthyroidism. Iodine in pharmacological doses was widely used to treat hyperthyroidism before the thionamide drugs (and radioiodine) became available. It was (and is) especially effective as a preparation for subsequent thyroid surgery, as introduced by Plummer in 1923 (379). However, escape from the effect during prolonged therapy was not uncommon, especially in patients with severe hyperthyroidism (380), and iodine therapy is now mostly used to reduce thyroid blood flow before surgery and as part of the combination of therapies given to patients with thyrotoxic crises.

An exception to this narrow indication for the treatment of hyperthyroidism is Japan, where there is considerable experience with high-dose iodine therapy in pregnancy $(381,382)$. The doses of iodine used have varied between 5 and $75 \mathrm{mg} / \mathrm{d}$, adjusted according to thyroid function tests. In a recent retrospective nonrandomized study, substituting MMI with potassium iodine in early pregnancy reduced the risk of birth defects. However, a proportion of such pregnant women became hyperthyroid following the change in treatment (382). Important to note, Japan is a high iodine intake country, and the Japanese results may not be reproducible in other countries. Cases of iodine-induced congenital hypothyroidism have been reported in children of U.S. women treated with high-dose iodine during pregnancy (383), and the use of iodine-containing disinfectants during labor is a well-established cause of elevated TSH during screening for congenital hypothyroidism (384). Thus, at present, such therapy cannot be recommended outside Japan until more evidence on safety and efficacy is available.

Theoretically, other possible treatments can also be considered. Cholestyramine is not absorbed from the gut (and thus not transferred to the fetus) and may moderately reduce circulating thyroid hormones by binding the hormones during their enterohepatic circulation (385-387). Cholestyramine has been used in pregnancy to treat obstetric cholestasis (388). Gastrointestinal discomfort with nausea, vomiting, and diarrhea may be a problem (388), and there is a risk of binding in the gut of vitamins and other substances important in pregnancy. Perchlorate competitively inhibits the active transport of iodide into the thyroid (and into breast milk), and it has been used to treat hyperthyroidism $(389,390)$. Limited evidence suggests it has no teratogenic effects as long as normal maternal thyroid function is maintained (391). In summary, more data are needed before the use of any such drugs can be recommended. Lithium has thyroid-inhibiting effects somewhat similar to pharmacological doses of iodine (392), but it may be teratogenic (393) and therefore should not be used to treat hyperthyroidism in pregnancy.

\section{- RECOMMENDATION 45}

Women taking MMI or PTU should be instructed to confirm potential pregnancy as soon as possible. If the pregnancy test is positive, pregnant women should contact their caregiver immediately.

\section{Strong recommendation, high-quality evidence.}




\section{- RECOMMENDATION 46}

(a) In a newly pregnant woman with GD, who is euthyroid on a low dose of MMI $(\leq 5-10 \mathrm{mg} / \mathrm{d})$ or PTU $(\leq 100$ $200 \mathrm{mg} / \mathrm{d}$ ), the physician should consider discontinuing all antithyroid medication given potential teratogenic effects. The decision to stop medication should take into account the disease history, goiter size, duration of therapy, results of recent thyroid function tests, TRAb measurement, and other clinical factors.

Weak recommendation, low-quality evidence.

(b) Following cessation of antithyroid medication, maternal thyroid function testing (TSH, and FT4 or TT4) and clinical examination should be performed every 1-2 weeks to assess maternal and fetal thyroid status. If the pregnant woman remains clinically and biochemically euthyroid, test intervals may be extended to 2-4 weeks during the second and third trimester.

Weak recommendation, low-quality evidence.

(c) At each assessment, the decision to continue conservative management (withholding antithyroid medication) should be guided both by the clinical and the biochemical assessment of maternal thyroid status.

Weak recommendation, low-quality evidence.

\section{- RECOMMENDATION 47}

In pregnant women with a high risk of developing thyrotoxicosis if antithyroid drugs were to be discontinued, continued antithyroid medication may be necessary. Factors predicting high clinical risk include being currently hyperthyroid, or requirement of $>5-10 \mathrm{mg} / \mathrm{d}$ MMI or $>100-200 \mathrm{mg} / \mathrm{d}$ PTU to maintain a euthyroid state. In such cases,

(a) PTU is recommended for the treatment of maternal hyperthyroidism through 16 weeks of pregnancy.

Strong recommendation, moderate-quality evidence.

(b) Pregnant women receiving MMI who are in need of continuing therapy during pregnancy should be switched to PTU as early as possible.

Weak recommendation, low-quality evidence.

(c) When shifting from MMI to PTU, a dose ratio of approximately 1:20 should be used (e.g., MMI $5 \mathrm{mg}$ / $\mathrm{d}=$ PTU $50 \mathrm{mg}$ twice daily).

Strong recommendation, moderate-quality evidence.

(d) If ATD therapy is required after 16 weeks gestation, it remains unclear whether PTU should be continued or therapy changed to MMI. As both medications are associated with potential adverse effects and shifting potentially may lead to a period of less-tight control, no recommendation regarding switching antithyroid drug medication can be made at this time.

No recommendation, insufficient evidence.

\section{Question 56: What are the principles of thyroid testing and ATD administration when treating Graves' hyperthyroidism during pregnancy?}

Thyroid stimulating antibodies, ATDs, and most maternal thyroid hormones can effectively cross the placenta barrier. When the fetal thyroid is functional, it can respond to TRAb antibodies, causing excess fetal production of thyroid hormone. Furthermore, if the mother has an intact thyroid and is hyperthyroid from GD, the fetus will also be exposed to the hyperthyroxinemia produced by the mother's thyroid during gestation. Typically, fetal hyperthyroidism due to crossplacental passage of TRAb develops at or after week 20 of pregnancy. However, one case has been published in which excessive maternal TRAb production led to fetal hyperthyroidism by week 18 (394).

As mentioned, MMI, PTU, and CM also effectively cross the placenta, and therefore ATD therapy for maternal hyperthyroidism also modulates fetal thyroid function. Importantly, all ATDs tend to be more potent in the fetus than in the mother. Thus, when the mother is made euthyroid, the fetus is often overtreated (395). Therefore, in order to avoid a deleterious fetal impact, the aim of treatment is to maintain maternal TT4/ FT4 values at, or just above the pregnancy-specific upper limit of normal. As a general rule, the smallest possible dose of ATDs should be used whenever possible.

In the setting of hyperthyroidism during pregnancy, maternal TT4/FT4 and TSH (and in cases of severe hyperthyroidism, also serum $\mathrm{T} 3$ ) should be measured approximately every 2-4 weeks following initiation of therapy, and every 4 6 weeks after achieving the target value (396-398). When trimester-specific FT4 values are not available, use of the reference range for nonpregnant patients is recommended. Separately, a TT4 measurement with reference value 1.5 times the nonpregnancy range may be used in second and third trimesters. Overtreatment should be avoided because of the possibility of inducing fetal goiter and or fetal hypothyroidism (399).

In the first trimester of pregnancy some women with GD experience an exacerbation of symptoms (400), which is parallel to the moderate increase in incidence of GD in early pregnancy (401). By the third trimester the incidence of GD becomes very low (401) corresponding to the general decrease in thyroid autoimmunity with a decrease in TRAb.

Discontinuation of all ATD therapy is feasible in $20 \%-$ $30 \%$ of patients in the last trimester of gestation (402). Maternal serum TSH well within the reference range is a sign that the ATD dose has to be reduced to avoid fetal overtreatment. If this is not done, fetal hypothyroidism and goiter may develop from overtreatment with ATDs. Disappearance of maternal TRAb in late pregnancy indicates a high likelihood of successful ATD withdrawal.

\section{- RECOMMENDATION 48}

(a) In women being treated with ATDs in pregnancy, FT4/ TT4 and TSH should be monitored approximately every 4 weeks.

Strong recommendation, moderate-quality evidence.

(b) Antithyroid medication during pregnancy should be administered at the lowest effective dose of MMI or PTU, targeting maternal serum FT4/TT4 at the upper limit or moderately above the reference range.

Strong recommendation, high-quality evidence.

Because the fetal thyroid responds more strongly to ATD therapy than the maternal thyroid, mothers on an ATD in the second half of pregnancy, who by nonpregnancy standards would be considered euthyroid, should have the ATD dose reduced to protect the fetus.

In occasional patients, GD stays very active and serum TT3 may remain elevated even if TT4/FT4 becomes normal 
or even low (403). An increase in ATD dose to normalize maternal serum TT3 will cause elevated serum TSH in the infants at birth (398), and a balance in ATD dosing with careful clinical evaluation of the fetus and the mother is needed.

Worsening of disease activity with a need for an increase in ATD dose or relapse of previously remitted disease often occurs after delivery $(400,404)$. Women should be informed about this risk, and appropriate monitoring performed. A single Japanese study has suggested that relapse may be prevented by low-dose ATD during the postpartum period (405), but more studies on this are needed.

The combination of LT4 and an ATD (block-replace therapy) has in general been shown not to improve GD remission rates (406), and it results in a larger dose of ATD required in order to maintain the FT4 within the target range. The placenta is readily permeable to the ATD but not to the LT4 given to the mother, and the fetal thyroid is relatively more sensitive to the effect of ATDs than the maternal thyroid. Therefore, block-replacement therapy given to the mother in the second half of pregnancy will generally lead to fetal goiter and hypothyroidism (403). The only indication for such combination therapy during pregnancy is in the treatment of isolated fetal hyperthyroidism caused by maternal TRAb production in a mother who previously received ablative therapy for GD (407). The ATD will pass the placenta and treat the fetal hyperthyroidism, whereas the LT4 is necessary to keep the mother euthyroid.

\section{- RECOMMENDATION 49}

A combination regimen of LT4 and an ATD should not be used in pregnancy, except in the rare situation of isolated fetal hyperthyroidism.

\section{Strong recommendation, high-quality evidence.}

\section{Question 57: What are the indications and timing for thyroidectomy in the management of GD during pregnancy?}

Thyroidectomy should be considered in cases of allergies/contraindications to both ATDs, in the patient who is not compliant with drug therapy, and in women in whom euthyroidism cannot be achieved even on large doses of ATDs. If surgery is indicated, the second trimester is the optimal time. Thyroidectomy is often followed by a gradual, but not immediate disappearance of TRAb, and withdrawal of ATD in the mother after thyroidectomy may lead to isolated fetal hyperthyroidism (403). High serum TRAb values before surgery indicate a risk for isolated fetal hyperthyroidism, and after maternal ATD withdrawal a program of careful fetal monitoring and possible therapy should be planned (403). In hyperthyroid patients not tolerating or nonresponsive to ATD therapy, preparation for surgery with $\beta$-blocking agents and a short course of potassium iodide solution $(50-100 \mathrm{mg} / \mathrm{d})$ are recommended (381). Potassium iodide preparation before surgery is also recommended in patients with other signs of active disease to reduce bleeding during surgery.

\section{- RECOMMENDATION 50}

Thyroidectomy in pregnancy may be indicated for unique scenarios. If required, the optimal time for thyroidectomy is in the second trimester of pregnancy. If maternal TRAb concentration is high ( $>3$ times the upper reference for the assay) the fetus should be carefully monitored for development of fetal hyperthyroidism throughout pregnancy, even if the mother is euthyroid post thyroidectomy.

\section{Strong recommendation, high-quality evidence.}

\section{Question 58: How should pregnant patients with $G D$ be prepared for urgent nonthyroid surgery?}

\section{- RECOMMENDATION 51}

We concur with the American College of Obstetricians and Gynecologists' Committee on Obstetric Practice consensus guidelines (written in 2011 and revised in 2015) (408) which state the following: "1) A pregnant woman should never be denied indicated surgery, regardless of trimester. 2) Elective surgery should be postponed until after delivery. 3) If possible, nonurgent surgery should be performed in the second trimester when preterm contractions and spontaneous abortion are least likely.',

In the setting of a patient with GD undergoing urgent, nonthyroid surgery, if the patient is well controlled on ATD, no other preparation is needed. Beta-blockade should also be utilized if needed.

Strong recommendation, moderate-quality evidence.

\section{Question 59: What is the value of TRAb measurement in the evaluation of a pregnant woman with Graves' hyperthyroidism?}

Fetal risks in women with previous or current Graves' hyperthyroidism include (a) fetal hyperthyroidism, (b) neonatal hyperthyroidism, (c) fetal hypothyroidism, (d) neonatal hypothyroidism, and (e) central hypothyroidism. The above potential complications depend on several factors: (a) poor control of hyperthyroidism throughout pregnancy may induce transient central hypothyroidism $(409,410)$; (b) excessive amounts of ATDs may be responsible for fetal and neonatal hypothyroidism, even if the mother is biochemically euthyroid $(399,403)$; and (c) high levels of thyroid stimulating antibodies in the second half of pregnancy may induce fetal and neonatal hyperthyroidism $(411,412,413,414)$. TRAb is measurable in around $95 \%$ of patients with active Graves' hyperthyroidism, and levels may remain high following ablation therapy, even more so after radioiodine treatment than surgical removal (415).

Indications for ordering a TRAb test in pregnant women with GD include (a) mothers with untreated or ATD-treated hyperthyroidism in pregnancy, (b) a previous history of GD with past treatment with radioiodine or total thyroidectomy, (c) a previous history of delivering an infant with hyperthyroidism, or (d) a known history of thyroidectomy for the treatment of hyperthyroidism in pregnancy (416). In the majority of patients, maternal TRAb concentrations decrease with the progression of pregnancy; however, as in nonpregnant patients, the course of GD is variable.

The incidence of fetal and neonatal hyperthyroidism is between $1 \%$ and $5 \%$ in all women with active or a past history of Graves' hyperthyroidism and is associated with increased fetal/neonatal morbidity and mortality if 
unrecognized and untreated (417). In a recent French study (418), follow-up of 47 newborns of mothers harboring measurable levels of TRAb in pregnancy showed that nine developed transient neonatal hyperthyroidism and five of these ( $9 \%$ overall) had to be treated with ATD for a median duration of 60 days. A maternal TRAb serum concentration $>5$ IU/L (approximately 3 times the upper limit of normal for the assay) in the second and third trimester predicted neonatal hyperthyroidism with $100 \%$ sensitivity and $43 \%$ specificity. A similar TRAb level risk cutoff was found in a separate French study (419).

A determination of serum TRAb in early pregnancy is helpful in detecting pregnancies at risk. A value $>5$ IU/L or 3 times the upper limit of normal in a mother who previously received ablative therapy for GD is an indication for establishing close follow-up of the fetus in collaboration with a maternal-fetal medicine physician. Cases of overlooked isolated fetal hyperthyroidism leading to fetal loss in such women have repeatedly been published.

A determination of serum TRAb in late pregnancy in a mother who is still in need of ATD therapy to remain euthyroid, is helpful for detecting risk for neonatal hyperthyroidism. A value $>5 \mathrm{IU} / \mathrm{L}$ or 3 times the upper limit of normal in the mother indicates that the fetal thyroid may be strongly stimulated by TRAb passing through the placenta. After birth, any ATD from the mother is much more rapidly cleared in the neonate than are the TRAb, and the neonate may become hyperthyroid.

If TRAb becomes undetectable in a pregnant woman taking ATD, it may be feasible to reduce or withdraw the ATD to protect the fetus against hypothyroidism and goiter.

In women who are in remission and euthyroid after a previous course of ATD therapy for GD, measurement of $\mathrm{TRAb}$ in pregnancy is not required (416).

\section{- RECOMMENDATION 52}

(a) If the patient has a past history of GD treated with ablation (radioiodine or surgery), a maternal serum determination of TRAb is recommended at initial thyroid function testing during early pregnancy.

Strong recommendation, moderate-quality evidence.

(b) If maternal TRAb concentration is elevated in early pregnancy, repeat testing should occur at weeks 18-22. Strong recommendation, moderate-quality evidence.

(c) If maternal TRAb is undetectable or low in early pregnancy, no further TRAb testing is needed.

Weak recommendation, moderate-quality evidence.

(d) If a patient is taking ATDs for treatment of Graves' hyperthyroidism when pregnancy is confirmed, a maternal serum determination of TRAb is recommended. Weak recommendation, moderate-quality evidence.

(e) If the patient requires treatment with ATDs for GD through midpregnancy, a repeat determination of TRAb is again recommended at weeks 18-22.

Strong recommendation, moderate-quality evidence.

(f) If elevated TRAb is detected at weeks 18-22 or the mother is taking ATD in the third trimester, a TRAb measurement should again be performed in late pregnancy (weeks 30-34) to evaluate the need for neonatal and postnatal monitoring.

Strong recommendation, high-quality evidence.
Question 60: Under what circumstances should additional fetal ultrasound monitoring for growth, heart rate, and goiter be performed in women with Graves' hyperthyroidism in pregnancy?

Serial ultrasound examinations may be performed for the assessment of gestational age, fetal viability, amniotic fluid volume, fetal anatomy, and detection of malformations. Fetal well-being may be compromised in the presence of elevated TRAb, uncontrolled hyperthyroidism, and pre-eclampsia (349,420-422). Signs of potential fetal hyperthyroidism that may be detected by ultrasonography include fetal tachycardia (heart rate $>170 \mathrm{bpm}$, persistent for over 10 minutes), intrauterine growth restriction, presence of fetal goiter (the earliest sonographic sign of fetal thyroid dysfunction), accelerated bone maturation, signs of congestive heart failure, and fetal hydrops $(411,421-423)$. A team approach to the management of these patients is required, including an experienced obstetrician or maternal-fetal medicine specialist, neonatologist, and anesthesiologist. In most cases, the diagnosis of fetal hyperthyroidism should be made on clinical grounds based on maternal history, interpretation of serum TRAb levels, and fetal ultrasonography $(396,411,422,423)$.

\section{- RECOMMENDATION 53}

Fetal surveillance should be performed in women who have uncontrolled hyperthyroidism in the second half of pregnancy, and in women with high TRAb levels detected at any time during pregnancy (greater than 3 times the upper limit of normal). A consultation with an experienced obstetrician or maternal-fetal medicine specialist is recommended. Monitoring may include ultrasound to assess heart rate, growth, amniotic fluid volume, and the presence of fetal goiter.

\section{Strong recommendation, moderate-quality evidence.}

\section{Question 61: When should umbilical blood sampling be considered in women with GD in pregnancy?}

Umbilical cord blood sampling (cordocentesis) is associated with both fetal mortality and morbidity $(424,425)$. It has been utilized when a mother is TRAb positive and treated with ATDs, a fetal goiter is present, and the thyroid status of the fetus is unclear $(403,411,426)$. The presence of elevated TRAb alone is not an indication for cordocentesis (427).

\section{- RECOMMENDATION 54}

Cordocentesis should be used in rare circumstances and performed in an appropriate setting. It may occasionally be of use when fetal goiter is detected in women taking ATDs to help determine whether the fetus is hyperthyroid or hypothyroid.

\section{Weak recommendation, low-quality evidence.}

Recently, biological assays able to distinguish between stimulating and blocking TRAb have become commercially available (428). The use of such assays for characterization of TRAb biological activity may theoretically make cordocentesis unnecessary, though the complexity of differential clearance of stimulatory and blocking antibodies should be considered (see Section X, Fetal and Neonatal Considerations). 


\section{Question 62: How should hyperthyroidism caused by autonomous thyroid nodules be handled in pregnancy?}

One or more autonomous thyroid nodules is a common cause of hyperthyroidism in populations with current or previous mild to moderate iodine deficiency (429). However, even in such areas this type of hyperthyroidism is quite rare under the age of 40 years (430). Hyperthyroidism caused by autonomous nodules tends to develop more insidiously and be less severe than in GD.

In pregnancy, a major difference from GD is that no TRAb is produced by the mother, and consequently the fetal thyroid is not stimulated as it is in GD. Therefore, ATD therapy to make the mother euthyroid would significantly increase the risk of hypothyroidism and goiter in the fetus. Given the limited data on this subject, if ATDs are required in this setting, careful monitoring of the fetus should occur. Furthermore, the dose of ATD should be kept low, and maternal surgical therapy with removal of autonomous nodule(s) considered if signs of fetal hypothyroidism develop. Generally, if possible, ablative therapy should be considered before conception for hyperthyroidism caused by thyroid autonomy in women seeking future pregnancy.

If only low-grade autonomous thyroid hormone production with subclinical hyperthyroidism is present in a nonpregnant woman, the physiological increase in thyroid hormone needs may ameliorate any hyperthyroidism in pregnancy. On the other hand, the early pregnancy high hCG level may theoretically activate nonaffected normal thyroid tissue and increase thyroid secretion in early pregnancy. Furthermore, hormone production in autonomous thyroid nodules will depend on the available amount of iodine. Therefore, women with such disease should probably refrain from taking iodine-containing supplements in pregnancy.

\section{- RECOMMENDATION 55}

If ATD therapy is given for hyperthyroidism caused by autonomous nodules, the fetus should be carefully monitored for goiter and signs of hypothyroidism during the second half of pregnancy. A low dose of ATD should be administered with the goal of maternal FT4 or TT4 concentration at the upper limit or moderately above the reference range.

Strong recommendation, low-quality evidence.

\section{Question 63: What are the etiologies of thyrotoxicosis in the postpartum period?}

The most common cause of thyrotoxicosis in the postpartum period is postpartum thyroiditis (PPT), with a thyrotoxicosis prevalence of $4 \%(431,432)$ (see Section XII). Many cases are mild and of short duration and spontaneously revert to euthyroidism. More severe cases in need of a short course of $\beta$-blockers are seen in patients with high levels of TPOAb, and they are often followed by a period of hypothyroidism. The majority of women return to euthyroidism by 1 year post partum $(433,434)$.

The postpartum increase in thyroid autoimmunity is also associated with a 3- to 4-fold increase in the incidence of new GD (401), in addition to the increase in the risk of relapse of GD in remission after previous ATD therapy. In one study, the overall relapse rate of GD following a pregnancy was
$84 \%$, as compared to a relapse rate of $56 \%$ in women who did not become pregnant (404).

\section{Question 64: How should Graves' hyperthyroidism be treated in lactating women?}

See Section XI.

\section{THYROID NODULES AND THYROID CANCER DURING PREGNANCY}

Thyroid nodules and thyroid cancer discovered during pregnancy present unique challenges to both the clinician and the mother. A careful balance is required between making a definitive diagnosis and instituting treatment while avoiding interventions that may adversely impact the mother, the health of the fetus, or the maintenance of the pregnancy.

\section{Question 65: What is the prevalence of thyroid nodules during pregnancy?}

Three studies have evaluated the prevalence of thyroid nodules during pregnancy, the impact of pregnancy on nodular size, and the proportion of women who have new nodules detected during pregnancy. All three studies were performed in areas with mild to moderate iodine deficiency (Brussels, China, and Germany), with the majority using ultrasound examination of the gland. The prevalence of thyroid nodules varied between $3 \%$ and $21 \%(187,435,436)$ and increased with increasing parity $(9.4 \%$ without a prior pregnancy, $20.7 \%$ with one prior pregnancy, $20.7 \%$ with two prior pregnancies, and $33.9 \%$ with three or more prior pregnancies) (435). In the Belgian study, $60 \%$ of nodules doubled in size during pregnancy, yet remained between 5 and $12 \mathrm{~mm}$ (187). The maximum diameter of the dominant nodule did not increase during pregnancy in the Chinese study (mean diameter was $5.1 \mathrm{~mm}$, $5.1 \mathrm{~mm}$, and $5.5 \mathrm{~mm}$, in the first, second, and third trimester, respectively). However, an increase in nodular volume was reported during pregnancy, with a return to first-trimester volumes by the third postpartum month (436). The studies in Belgium and Germany reported that $11 \%-20 \%$ of women with a nodule detected in the first trimester of pregnancy developed a second nodule through the course of pregnancy $(187,436)$. Increasing age is associated with an increase in the proportion of pregnant women who have thyroid nodules $(187,436)$.

\section{Question 66: What is the prevalence of thyroid cancer in women with thyroid nodules discovered during pregnancy?}

Data regarding the prevalence of thyroid cancer derive from three retrospective studies performed at three tertiary referral centers (Mayo Clinic, George Washington University Hospital, and Mount Sinai Hospital-Toronto), one prospective study, and a retrospective study of the California Cancer Registry. The research performed at the referral centers revealed a 15\% (6/40, Mayo Clinic) (437), 12\% (7/57, George Washington Hospital) (438), and 43\% (7/16, Mount Sinai Hospital-Toronto) (439) rate of thyroid malignancy. All three studies are limited by two major methodological flaws. The first, and most problematic, is selection bias. The population studies consisted of women referred for diagnosis and treatment at major referral centers. As such, they are not representative of the population of pregnant women with 
thyroid nodules detected during pregnancy. Instead, they represent a select group of women referred to a tertiary medical center because of physician concern for thyroid malignancy. Consequently, they likely represent an overrepresentation of the prevalence of thyroid malignancy during pregnancy. Secondly, each study was retrospective in nature and therefore neither the accuracy of the diagnosis nor the completeness of case identification within the database could be verified. The lone prospective study investigated the prevalence of thyroid cancer during pregnancy in $212 \mathrm{Chi}-$ nese women. The study found a $15.3 \%$ (34/221) rate of thyroid nodules and a $0 \%$ rate of malignancy. Interpreting these data is hampered by the limited number of women enrolled in the study (436). The final study consisted of a populationbased retrospective analysis of all obstetrical deliveries in California from 1991 through 1999 identified by crossreferencing maternal/neonatal hospital discharges in California and the California Cancer Registry $(n=4,846,505$ women). A prevalence of thyroid cancer in pregnancy of 14.4/100,000 was reported, with papillary cancer being the most frequent pathological type (440). Timing of diagnosis of the thyroid malignancy was as follows: $3.3 / 100,000$ cases diagnosed before delivery, 0.3/100,000 at delivery, and 10.8/ 100,000 within 1 year post partum.

\section{Question 67: What is the optimal diagnostic strategy for thyroid nodules detected during pregnancy?}

History and physical examination. The patient with a thyroid nodule should be asked about a family history of benign or malignant thyroid disease, familial medullary thyroid carcinoma, multiple endocrine neoplasia type 2 (MEN 2), familial papillary thyroid carcinoma, and a familial history of a tumor syndrome predisposing to thyroid cancer syndrome (e.g., phosphatase and tensin homolog [PTEN] hamartoma tumor syndrome [Cowden's disease], familial adenomatous polyposis, Carney complex, or Werner syndrome) $(441,442,443)$. The malignancy risk is higher for nodules detected in both adult survivors of childhood cancers in which treatment involved head, neck, and/or cranial radiation and those exposed to ionizing radiation before 18 years of age $(444,445)$. Thorough palpation of the thyroid and neck inspection for cervical nodes is essential (446).

Ultrasound. Thyroid ultrasound is the most accurate tool for detecting thyroid nodules, determining their sonographic features and pattern, monitoring growth, and evaluating cervical lymph nodes. The recent 2015 ATA Management Guidelines for Adult Patients with Thyroid Nodules and Differentiated Thyroid Cancer should be referenced for diagnostic use and performance of thyroid and neck sonography as well as for decision-making regarding fine-needle aspiration (FNA) for thyroid nodules (443). Recent reports have validated that the identification of defined nodule sonographic patterns representing constellations of sonographic features is more robust for malignancy risk correlation than that associated with individual ultrasound characteristics $(447,448)$. Hence, a high-suspicion sonographic pattern that includes solid hypoechoic nodules with irregular borders and microcalcifications correlates with a $>70 \%$ chance of cancer compared to the very low-suspicion pattern of a noncalcified mixed cystic solid or spongiform nodule $(<3 \%$ cancer risk). Recommendation 9 in the 2015 ATA guidelines (443) indicates different FNA size cutoffs based upon five defined sonographic patterns and their associated risk stratification for thyroid cancer (Table 9).

Thyroid function tests. All women with a thyroid nodule should have a TSH measurement performed $(449,450)$. Thyroid function tests are usually normal in women with thyroid cancer. In nonpregnant women, a subnormal serum TSH level may indicate a functioning nodule, which is then evaluated with scintigraphy because functioning nodules are

Table 9. Ata Sonographic Patterns and Estimated Risk of Malignancy for Thyroid Nodules (AdAPTED From ATA 2015 Guidelines With PERMission)

\begin{tabular}{|c|c|c|c|}
\hline $\begin{array}{l}\text { Sonographic } \\
\text { pattern }\end{array}$ & Ultrasound features & $\begin{array}{l}\text { Estimated risk } \\
\text { of malignancy }\end{array}$ & $\begin{array}{l}\text { FNA size cutoff } \\
\text { (largest dimension) }\end{array}$ \\
\hline $\begin{array}{l}\text { High } \\
\text { suspicion }\end{array}$ & $\begin{array}{l}\text { Solid hypoechoic nodule or solid hypoechoic component } \\
\text { of a partially cystic nodule with one or more of the } \\
\text { following features: irregular margins (infiltrative, } \\
\text { microlobulated), microcalcifications, taller than wide } \\
\text { shape, rim calcifications with small extrusive soft tissue } \\
\text { component, evidence of extrathyroidal extension }\end{array}$ & $>70 \%-90 \%$ & Recommend at $>1 \mathrm{~cm}$ \\
\hline $\begin{array}{l}\text { Intermediate } \\
\text { suspicion }\end{array}$ & $\begin{array}{l}\text { Hypoechoic solid nodule with smooth margins without } \\
\text { microcalcifications, extrathyroidal extension, or taller } \\
\text { than wide shape }\end{array}$ & $10 \%-20 \%$ & Recommend at $>1 \mathrm{~cm}$ \\
\hline $\begin{array}{l}\text { Low } \\
\quad \text { suspicion }\end{array}$ & $\begin{array}{l}\text { Isoechoic or hyperechoic solid nodule, or partially cystic } \\
\text { nodule with eccentric solid areas, without } \\
\text { microcalcification, irregular margin or extrathyroidal } \\
\text { extension, or taller than wide shape. }\end{array}$ & $5 \%-10 \%$ & Recommend at $>1.5 \mathrm{~cm}$ \\
\hline $\begin{array}{l}\text { Very low } \\
\text { suspicion }\end{array}$ & $\begin{array}{l}\text { Spongiform or partially cystic nodules without any of the } \\
\text { sonographic features described in low, intermediate } \\
\text { or high suspicion patterns }\end{array}$ & $<3 \%$ & $\begin{array}{l}\text { Consider at }>2 \mathrm{~cm} \text {; } \\
\text { observation without FNA } \\
\text { is also a reasonable option }\end{array}$ \\
\hline Benign & Purely cystic nodules (no solid component) & $<1 \%$ & No biopsy \\
\hline
\end{tabular}

Ultrasound-guided fine-needle aspiration (FNA) is recommended for lateral neck cervical lymph nodes that are sonographically suspicious for thyroid cancer. 
so rarely malignant that cytologic evaluation is not indicated. However, pregnancy produces two hurdles for following this algorithm. First, the lower limit of the TSH reference range decreases, especially during early gestation, making it difficult to differentiate what is normal for pregnancy from potential nodular autonomous function. Second, scintigraphy with either technetium pertechnetate or ${ }^{123} \mathrm{I}$ is contraindicated in pregnancy (see below).

Calcitonin and thyroglobulin. As within the general population, the routine measurement of calcitonin remains controversial (451). Calcitonin measurement may be performed in pregnant women with a family history of medullary thyroid carcinoma or MEN 2 or a known RET gene mutation. However, the utility of measuring calcitonin in all pregnant women with thyroid nodules has not been evaluated. The pentagastrin stimulation test is contraindicated in pregnancy (452).

In the presence of an in situ thyroid gland, serum $\mathrm{Tg}$ measurements are neither sensitive nor specific for thyroid cancer and can be elevated in many benign thyroid disorders (453). Thus, serum Tg measurement is not recommended.

Fine-needle aspiration. Fine needle aspiration is a safe diagnostic tool in pregnancy and may be performed in any trimester (454-463). Two retrospective case series of FNAs performed during pregnancy, involving a total of 94 patients, have been published. In those cases in which surgery was performed, pathological examination of the specimens confirmed the diagnosis of all FNAs classified by cytology as either benign or malignant. Six of the $16(37.5 \%)$ cases reported by cytology as suspicious for malignancy were found to be malignant at pathological examination $(437,438)$. Pregnancy does not appear to alter a cytological diagnosis of thyroid tissue obtained by FNA, but there have been no prospective studies to evaluate potential differences in FNA cytology obtained during pregnancy versus in the nonpregnant state. Since overall survival does not differ if surgery is performed during or after gestation in patients diagnosed with thyroid cancer during pregnancy (464-466), patient preference for timing of FNA (during pregnancy or post partum) should be considered.

Radionuclide scanning. Iodine 131 readily crosses the placenta and the fetal thyroid begins to accumulate iodine by 12-13 weeks gestation (467). There are reports of inadvertent administration of therapeutic ${ }^{131} \mathrm{I}$ therapy for treatment of hyperthyroidism during unsuspected pregnancy. If ${ }^{131} \mathrm{I}$ is given after 12-13 weeks gestation, it accumulates in the fetal thyroid resulting in fetal/neonatal hypothyroidism. In this scenario, the International Atomic Energy Agency (IAEA) recommends intervention with $60-130 \mathrm{mg}$ of stable potassium iodide given to the mother only if the pregnancy is discovered within 12 hours of ${ }^{131} \mathrm{I}$ administration. This will partially block the fetal thyroid, hence reducing fetal thyroid ${ }^{131} \mathrm{I}$ uptake (468). However, if maternal treatment occurs prior to 12 weeks, the fetal thyroid does not appear to be damaged (469471). Rather, the issue is the fetal whole-body radiation dose due to gamma emissions from ${ }^{131} \mathrm{I}$ in the maternal bladder, which is in the range of $50-100 \mathrm{mGy} / \mathrm{GBq}$ of administered activity. This dose is decreased by hydrating the mother and by encouraging frequent voiding (468). No studies have specifically examined whether scanning doses of ${ }^{123} \mathrm{I}$ or technetium pertechnetate have adverse fetal effects if used during gesta- tion. In general, these are contraindicated because all maternal radionuclides are associated with a fetal irradiation resulting from both placental transfer and external irradiation from maternal organs, specifically the bladder. Again, both maternal hydration and frequent voiding reduce fetal exposure (468).

The optimal diagnostic strategy for thyroid nodules detected during pregnancy is based on risk stratification. All women should have the following: a complete history and clinical examination, serum TSH measurement, and ultrasound of the neck.

\section{- RECOMMENDATION 56}

For women with suppressed serum TSH levels that persist beyond 16 weeks gestation, FNA of a clinically relevant thyroid nodule may be deferred until after pregnancy. At that time, if serum TSH remains suppressed, a radionuclide scan to evaluate nodule function can be performed if not breastfeeding.

\section{Strong recommendation, low-quality evidence.}

\section{- RECOMMENDATION 57}

The utility of measuring calcitonin in pregnant women with thyroid nodules is unknown. The task force cannot recommend for or against routine measurement of serum calcitonin in pregnant women with thyroid nodules.

\section{No recommendation, insufficient evidence.}

\section{- RECOMMENDATION 58}

Thyroid nodule FNA is generally recommended for newly detected nodules in pregnant women with a nonsuppressed TSH. Determination of which nodules require FNA should be based upon the nodule's sonographic pattern as outlined in Table 9. The timing of FNA, whether during gestation or early postpartum, may be influenced by the clinical assessment of cancer risk or by patient preference.

\section{Strong recommendation, moderate-quality evidence.}

\section{- RECOMMENDATION 59}

Radionuclide scintigraphy or radioiodine uptake determination should not be performed during pregnancy.

\section{Strong recommendation, high-quality evidence.}

\section{Question 68: Does pregnancy impact the prognosis of newly diagnosed thyroid carcinoma?}

Six studies have compared the diagnostic features and prognosis of women diagnosed with differentiated thyroid cancer (DTC) either during gestation or within the first year post partum to nonpregnant women (464-466,472-474). None of the studies were RCTs, all were retrospective, and the size of many of the studies was limited. Four studies either predate or do not use the contemporary tools for recurrence detection, including sensitive serum $\mathrm{Tg}$ assays and neck sonography (464-466,473). Using large database evaluation (464-466), the analyzed outcomes were either overall survival or diseasefree survival, neither of which differed between the two groups. This outcome is not unexpected because the vast majority ( $>99 \%$ ) of both the pregnant and nonpregnant populations had stage 1 disease. In addition, all studies consistently found that timing of surgery, either during pregnancy or deferred until postpartum, did not affect survival. 
Recently, using both sonography and sensitive Tg assays, two studies from referral centers in Italy reported higher persistence or recurrence rates in women diagnosed with thyroid cancer during pregnancy or within 12-24 months of delivery compared to both nulliparous women and those diagnosed after a longer interval post partum $(472,474)$. DTC persistence or recurrence was defined as (a) either stimulated or suppressed serum $\mathrm{Tg}>2 \mathrm{ng} / \mathrm{mL}$, (b) increasing levels of $\mathrm{Tg}$ autoantibodies, (c) sonography detection of metastatic lymph nodes, or (d) uptake outside the thyroid bed on scintigraphy. Subjects were followed for a median of about 6 years after definitive therapy. Vannucchi et al. (472) observed a much higher than expected persistence/recurrence rate $(60 \%)$ in the pregnant/early postpartum group than otherwise anticipated for Stage 1 disease, compared with $4 \%$ in women diagnosed more than 12 months after delivery and $13 \%$ in nulliparous women. The majority of the recurrences $(67 \%)$ were identified by $\mathrm{Tg}$ or $\mathrm{TgAb}$ criteria without identification of structural disease, so it is unclear if $\mathrm{Tg} / \mathrm{TgAb}$ detection is associated with other confounding factors such as potentially lessaggressive surgery if done during gestation. Messuti et al. (474) reported a recurrence rate of $10 \%$ that was more typical for early stage disease, but this was statistically higher than the $1.3 \%$ for women diagnosed 2 years after delivery and $4.3 \%$ for nulliparous women. No details for recurrence classification, biochemical versus structural, were provided. Importantly, delaying surgery until after delivery did not further affect recurrence rates among women diagnosed with DTC during gestation in either study. Although several potential explanations exist for the poorer prognosis of pregnant/early postpartum women versus the comparator groups, findings are not consistent in the two studies. Metastatic cervical adenopathy at diagnosis was more frequent in pregnant/postpartum women compared to the other groups in one study (63\% vs. 40\%) (472) but not in the other (474). Vannucchi et al. (472) documented more frequent presence of estrogen receptor $\alpha$ in the tumors of women diagnosed during pregnancy/early post partum as compared to the other two groups, which may indicate that the poorer prognosis is related to estrogen-mediated growth and stimulation of the MAPK pathway. However, this was not confirmed in the study by Messuti et al. (474). The discrepancy may be due to methodologic issues because a less-dilute estrogen receptor $\mathrm{Ab}$ was employed in the study with the positive findings. Hence, there is a recent observation of higher persistence/ recurrence rates for women diagnosed with DTC during or immediately after pregnancy, without elucidation of pathophysiologic mechanisms. Further studies may help to clarify causality. Importantly, survival is not compromised.

The impact of pregnancy on women with medullary or anaplastic carcinoma is unknown.

\section{Question 69: What are the perioperative risks to mother and fetus of thyroid surgery during pregnancy?}

Surgery is the treatment of choice for DTC. As deferring surgery until postpartum has not been associated with a worse prognosis, it is imperative to assess maternal and neonatal complications before recommending an operation during pregnancy. Between 1986 and 2008, nine studies evaluated the impact of thyroidectomy during pregnancy on a total of
113 patients (study size ranged from 1 to 96$)(437,441,442$, 444,446,475-478). The majority, but not all, of the operations were performed in the second trimester. There were no maternal or fetal complications in any of the studies. A 2009 population-based study of nonfederal hospitals in the United States compared 201 pregnant women who underwent thyroid and parathyroid surgery during pregnancy with 31,155 similarly treated nonpregnant women. One hundred sixtyfive operations were thyroid related and $46 \%$ of the women had thyroid cancer. Pregnant patients had a higher rate of endocrine and general complications, longer lengths of stay, and higher hospital costs. The fetal and maternal complication rates were $5.5 \%$ and $4.5 \%$, respectively (479). Interpretation of the results of this study is difficult because there were substantial baseline differences between the two groups. Pregnant women were more likely to have either urgent or emergent admissions and had a higher percentage of government insurance. However, surgical volume was also an independent predictor of poor outcomes, so that if surgery is performed during pregnancy, an experienced surgeon is preferred. Recently, a study from Japan focused on 45 patients with DTC, of whom 24 had thyroidectomy during pregnancy (19 during the second trimester) and 21 had surgery after delivery. The groups were compared with regard to disease and other factors. There was no difference in recurrence rates in the mothers and there were no pregnancy losses or birth defects. The authors concluded that although thyroidectomy can be performed safely in the second trimester, surgery after delivery is recommended for most patients with nonaggressive DTC (480). Thus, in instances in which surgery during pregnancy is indicated or desired, it should be performed in the second trimester in order to minimize complications to both the mother and fetus (altered organogenesis and spontaneous abortion in the first trimester, preterm labor and delivery in the third trimester) (481), preferably by an experienced thyroid surgeon. The risk of post-thyroidectomy maternal hypothyroidism and hypoparathyroidism should also be considered.

\section{Question 70: How should cytologically benign thyroid nodules be managed during pregnancy?}

Although pregnancy is a risk factor for modest progression of nodular thyroid disease, there is no evidence demonstrating that LT4 is effective in decreasing the size or arresting the growth of thyroid nodules during pregnancy. Hence, LT4 suppressive therapy for thyroid nodules is not recommended during pregnancy. Nodules that were benign on FNA but show rapid growth or ultrasound changes suspicious for malignancy should be evaluated with a repeat FNA and be considered for surgical intervention. In the absence of rapid growth, nodules with biopsies that are benign do not require surgery during pregnancy (482).

\section{- RECOMMENDATION 60}

Pregnant women with cytologically benign thyroid nodules do not require special surveillance strategies during pregnancy and should be managed according to the 2015 ATA Management Guidelines for Adult Patients with Thyroid Nodules and Differentiated Thyroid Cancer.

Strong recommendation, moderate-quality evidence. 


\section{Question 71: How should cytologically indeterminate nodules be managed during pregnancy?}

There have been no prospective studies evaluating the outcome and prognosis of women with an FNA that is interpreted as either atypia of undetermined significance/follicular lesion of undetermined significance (AUS/FLUS), suspicious for follicular neoplasm (SFN), or suspicious for malignancy (SUSP). The reported malignancy rates associated with these cytologic diagnoses range from $6 \%$ to $48 \%$ for AUS/FLUS, $14 \%$ to $34 \%$ for SFN, and $53 \%$ to $87 \%$ for SUSP (483). Although molecular testing for cytologically indeterminate nodules is now being considered, no validation studies address application of these tests in pregnant women. It is theoretically possible that thyroid gestational stimulation may alter a nodule's gene expression and change diagnostic performance of molecular tests based upon RNA expression, whereas testing based upon either single base-pair DNA mutations or translocations would be less likely to be affected.

Since prognosis for DTC diagnosed during pregnancy is not adversely affected by performing surgery post partum, it is reasonable to defer surgery until following delivery. Because the majority of these women will have benign nodules, LT4 therapy during pregnancy is not recommended.

\section{- RECOMMENDATION 61}

Pregnant women with cytologically indeterminate (AUS/ FLUS, SFN, or SUSP) nodules, in the absence of cytologically malignant lymph nodes or other signs of metastatic disease, do not routinely require surgery while pregnant.

Strong recommendation, moderate-quality evidence.

\section{- RECOMMENDATION 62}

During pregnancy, if there is clinical suspicion of an aggressive behavior in cytologically indeterminate nodules, surgery may be considered.

Weak recommendation, low-quality evidence.

\section{- RECOMMENDATION 63}

Molecular testing is not recommended for evaluation of cytologically indeterminate nodules during pregnancy.

Strong recommendation, low-quality evidence.

\section{Question 72: How should newly diagnosed thyroid carcinoma be managed during pregnancy?}

The 2015 ATA guidelines (443) recommend that a nodule with cytology indicating papillary thyroid carcinoma discovered early in pregnancy should be monitored sonographically and, if either it grows substantially by 24 weeks gestation $(50 \%$ in volume and $20 \%$ in diameter in two dimensions), or if metastatic cervical lymph nodes are present, surgery should be considered in the second trimester. However, if it remains stable by midgestation, or if it is diagnosed in the second half of pregnancy, surgery may be performed after delivery. Surgery in the second trimester is an option if the DTC is advanced stage at diagnosis or if the cytology indicates medullary or anaplastic carcinoma.
If surgery is not performed, the utility of thyroid hormone therapy targeted to lower serum TSH levels to improve the prognosis of DTC diagnosed during gestation is not known. Because higher serum TSH levels may be correlated with a more advanced stage of cancer at surgery (484), if the patient's serum TSH is $>2 \mathrm{mU} / \mathrm{L}$, it may be reasonable to initiate thyroid hormone therapy to maintain the $\mathrm{TSH}$ between 0.3 and $2.0 \mathrm{mU} / \mathrm{L}$ for the remainder of gestation.

\section{- RECOMMENDATION 64}

PTC detected in early pregnancy should be monitored sonographically. If it grows substantially before 24-26 weeks gestation, or if cytologically malignant cervical lymph nodes are present, surgery should be considered during pregnancy. However, if the disease remains stable by midgestation, or if it is diagnosed in the second half of pregnancy, surgery may be deferred until after delivery.

Weak recommendation, low-quality evidence.

\section{- RECOMMENDATION 65}

The impact of pregnancy on women with newly diagnosed medullary carcinoma or anaplastic cancer is unknown. However, a delay in treatment is likely to adversely affect outcome. Therefore, surgery should be strongly considered, following assessment of all clinical factors.

\section{Strong recommendation, low-quality evidence.}

\section{Question 73: What are the TSH goals for pregnant women with previously treated thyroid cancer receiving LT4 therapy?}

Based on studies which have demonstrated a lack of maternal or neonatal complications from subclinical hyperthyroidism, it is reasonable to assume that the preconception degree of TSH suppression can be safely maintained throughout pregnancy. The appropriate level of TSH suppression depends upon preconception risk of residual or recurrent disease. According to the 2009 and 2015 ATA management guidelines for DTC $(443,482)$, and the ETA consensus (485), the serum TSH should be maintained indefinitely below $0.1 \mathrm{mU} / \mathrm{L}$ in patients with persistent structural disease. Target TSH values are first based upon initial risk of recurrence determined postoperatively (Table 12 of the 2015 ATA guidelines) and then are modified based upon classification of the patient's response to therapy as defined in Table 13 of the 2015 ATA guidelines. Hence, for a patient at initial high risk for recurrence, TSH suppression at or below $0.1 \mathrm{mU} / \mathrm{L}$ is recommended. If the patient then demonstrates an excellent response to therapy at one year with an undetectable suppressed serum Tg and negative imaging, the TSH target may rise to the lower half of the reference range.

The main challenge in caring for women with previously treated DTC is maintaining the TSH level within the preconception range. In a recent report (325), thyroid cancer patients were reported to require smaller LT4 dose increases than patients who had undergone thyroid ablation for benign thyroid disorders or patients with primary hypothyroidism. However, this outcome was because the nonpregnant serum level TSH was $<0.1 \mathrm{mU} / \mathrm{L}$ in $83 \%$ of the thyroid cancer patients prior to pregnancy and this target was not achieved during gestation. It is likely that if the prepregnancy TSH 
target level is maintained throughout gestation, the LT4 dosage increase will be similar to the $\sim 49 \%$ required in ablated hypothyroidism. Patients require careful monitoring of thyroid function tests in order to avoid hypothyroidism.

Thyroid function should be evaluated as soon as pregnancy is confirmed. The adequacy of LT4 treatment should be checked 4 weeks after any LT4 dose change. The same laboratory should be utilized to monitor TSH and Tg levels before, during, and after pregnancy.

\section{- RECOMMENDATION 66}

Pregnant women with thyroid cancer should be managed at the same TSH goal as determined preconception. TSH should be monitored approximately every 4 weeks until 16-20 weeks of gestation, and at least once between 26 and 32 weeks of gestation.

Strong recommendation, moderate-quality evidence.

\section{Question 74: What is the effect of therapeutic radioactive iodine treatment on subsequent pregnancies?}

Following surgery for DTC, many patients will receive an ablative dose of radioactive iodine. The possible deleterious effect of radiation on gonadal function and the outcome of subsequent pregnancies has been evaluated by Sawka et al. (486) and Garsi et al. (487); the latter collected 2673 pregnancies, 483 of which occurred after radioiodine treatment. Neither study found an increased risk of infertility, pregnancy loss, stillbirths, neonatal mortality, congenital malformations, preterm births, low birth weight, or death during the first year of life or cancers in offspring.

Radioiodine treatment may lead to suboptimal thyroid hormonal control during the month following administration. The potential adverse effect upon the pregnancy is described in Section VII. It therefore seems reasonable to wait a minimum of 6 months to ensure that thyroid hormonal control is stable before conceiving following radioiodine ablative therapy.

Iodine 131 may affect spermatogenesis. In one study following men after ${ }^{131}$ I therapy, there was a dose-dependent increase in follicle-stimulating hormone levels and a reduction in normokinetic sperm (488). Therefore, it seems prudent for a man who has received ${ }^{131}$ I to wait 120 days (the lifespan of sperm) after ${ }^{131} \mathrm{I}$ therapy before attempting to conceive.

\section{- RECOMMENDATION 67}

Pregnancy should be deferred for 6 months after a woman has received therapeutic radioactive iodine $\left({ }^{131} \mathrm{I}\right)$ treatment.

\section{Strong recommendation, low-quality evidence.}

\section{Question 75: What is the effect of tyrosine kinase inhibitors on pregnancy?}

Several tyrosine kinase inhibitor (TKI) medications are now FDA approved for the therapy of metastatic differentiated and medullary thyroid cancer. These include sorafenib, lenvatinib, and cabozantinib. All three drugs have demonstrated both teratogenicity and embryo toxicity in animals when administered at doses below the recommended human dose (489). There are no human studies. The FDA recom- mends that women be advised of the potential risk to the fetus with TKI medications. However, specific advisories vary by medication. Furthermore, the use of any TKI must be guided by an assessment of risks to benefits that is also affected by the stage of disease and recommended drug indications. For sorafenib, the FDA prescribing information counsels to avoid pregnancy while taking the drug. For lenvatinib, contraception is explicitly recommended. For cabozantinib, no additional warnings are listed (489).

\section{Question 76: Does pregnancy increase the risk of DTC recurrence?}

Five studies have evaluated the impact of pregnancy after a woman has been treated for DTC. Two were published five decades ago, predating contemporary tools for thyroid cancer surveillance (490,491). Still, Rosvoll et al. (490) demonstrated pregnancy did not accelerate tumor growth in the 22 women who had stable or slowly progressive metastatic disease. Hill et al. (491) found no difference in clinically detected thyroid cancer recurrence rates in 70 women who had one or more pregnancies following initial diagnosis of DTC and 109 women who had no subsequent pregnancies. A more recent study from Memorial Sloan Kettering reported on 36 women who became pregnant between 1997 and 2006, a median of 4.3 years after initial treatment for DTC (492). Although all had serum Tg testing, only 16 (44\%) had ultrasound imaging before and after delivery. Disease progression occurred in one of the two women who had metastatic cervical lymph nodes on baseline imaging (the lymph node grew from $2.3 \mathrm{~cm}$ to $2.7 \mathrm{~cm}$ ). The mean suppressed $\mathrm{Tg}$ after delivery was not significantly different than the prepartum value. However, eight women had postpartum $\mathrm{Tg}$ values more than $20 \%$ higher than before pregnancy (three with known disease, five with no clinical evidence of disease). No evidence of recurrence was detected in the early postpartum period in women with negative prepregnancy neck ultrasound and a suppressed serum $\mathrm{Tg}<1 \mathrm{ng} / \mathrm{mL}$. Two more recent studies $(493,494)$ routinely used neck sonography and sensitive Tg assays to evaluate cancer progression during pregnancy. No recurrence was observed in 64 Brazilian women (48 papillary cancer and 16 follicular cancer) who had suppressed Tg levels $<2 \mathrm{ng} / \mathrm{mL}$ and negative neck sonography prepregnancy. However, follow-up was limited to only 6 months after delivery (493). Using a definition of disease-free as a suppressed $\mathrm{Tg}<0.9 \mathrm{ng} / \mathrm{mL}$ and negative neck ultrasound, Hirsch and colleagues (494) followed 63 women who had given birth after receiving treatment for DTC (all papillary thyroid cancer) for a mean of 4.8 years. None of the 50 women categorized as disease-free had progression of disease. However, 6 of the 13 women (46\%) with persistent disease prepregnancy experienced progression. Metastatic cervical lymph nodes grew in $50 \%$ of those with known prepregnancy nodal disease, and new nodal metastases appeared in two of the seven women with negative baseline sonography.

Thus, pregnancy does not pose a risk for tumor recurrence in women without structural or biochemical disease present prior to the pregnancy. Therefore, women with an excellent response to therapy as defined by the 2015 ATA guidelines (443) do not require additional monitoring during gestation. However, pregnancy may represent a stimulus to thyroid 
cancer growth in patients with known structural (ATA 2015 structural incomplete response to therapy) or biochemical (ATA 2015 biochemical incomplete response to therapy) disease present at the time of conception and requires monitoring.

\section{Question 77: What type of monitoring should be performed during pregnancy in a patient who has already been treated for DTC prior to pregnancy?}

\section{- RECOMMENDATION 68}

Ultrasound and $\mathrm{Tg}$ monitoring during pregnancy is not required in women with a history of previously treated DTC with undetectable serum Tg levels (in the absence of Tg autoantibodies) classified as having no biochemical or structural evidence of disease prior to pregnancy.

Strong recommendation, moderate-quality evidence.

\section{- RECOMMENDATION 69}

Ultrasound and Tg monitoring should be performed during pregnancy in women diagnosed with well-differentiated thyroid cancer and a biochemically or structurally incomplete response to therapy, or in patients known to have active recurrent or residual disease.

Strong recommendation, moderate-quality evidence.

\section{Question 78: What type of monitoring should be performed during pregnancy in a patient who is under active surveillance for papillary thyroid microcarcinoma?}

Although FNA of subcentimeter nodules in the absence of suspicious cervical adenopathy is not recommended by the 2015 ATA guidelines (443), there are times when this has been performed with subsequent diagnosis of a papillary thyroid microcarcinoma (PTMC). In some situations, women with this diagnosis may have chosen active surveillance. Observation with active surveillance has been demonstrated to be a viable option for patients cytologically diagnosed with PTMC ( size $<1 \mathrm{~cm})$ in the absence of suspicious lymph nodes or extrathyroidal invasion by sonographic imaging (495). Although tumor growth $>3 \mathrm{~mm}$ and appearance of novel metastatic lymph nodes occurred respectively overall in $8 \%$ and $3.8 \%$ of a cohort of 1235 Japanese patients, progression was more likely in patients younger than 40 years of age. Therefore, the same group published their observations of nine women with PTMC who became pregnant during active surveillance and compared outcomes to 27 age-matched nonpregnant women. During pregnancy, growth $\geq 3 \mathrm{~mm}$ occurred more frequently in pregnant $(44 \%)$ than nonpregnant $(11 \%)$ women $(p=0.0497)$. There was no correlation between growth and gestational TSH or Tg levels, and novel metastatic cervical lymph nodes were not observed. Three of the four patients who experienced tumor growth had surgery post partum, but the tumor decreased in size post partum in the fourth patient who is still under active surveillance. Furthermore, the PTMC enlarged by less than $3 \mathrm{~mm}$ in the other five pregnant women, but then decreased in three postpartum (496). This supports the hypothesis that pregnancy is either permissive for or promotes growth of papillary thyroid cancer. While this may have no impact on overall health, it seems reasonable to monitor such women with serial sonographic evaluations.

\section{- RECOMMENDATION 70}

Ultrasound monitoring of the maternal thyroid should be performed each trimester during pregnancy in women diagnosed with PTMC who are under active surveillance.

Weak recommendation, low-quality evidence.

\section{Question 79: What special considerations should be followed for women with medullary cancer due to germline RET mutations?}

Hereditary medullary thyroid cancer (MTC) may occur as a result of activating germline mutations of the RET oncogene. Over 100 mutations, duplications, insertions, or deletions involving RET have been identified in patients with hereditary MEN2a (including familial MTC) or MEN2b syndromes. The newly Revised American Thyroid Association Guidelines for the Management of Medullary Thyroid Carcinoma have graded the most common mutations according to risk of aggressive MTC and likelihood of concomitant pheochromocytoma and hyperparathyroidism (497). For a woman with a given RET mutation with or without clinical MTC, who is either pregnant or contemplating conception, the associated risks of MTC aggressiveness combined with her own history may inform her about whether to seek genetic counseling. Both prenatal and preimplantation genetic testing are available, and the reader is referred to the excellent discussion in the 2015 ATA guidelines that culminates in Recommendation 12: "The duty to warn of genetic risk extends to both preconception and prenatal contexts. Genetic counseling about the options of pre-implantation or prenatal diagnostic testing should be considered for all RET mutation carriers of childbearing age, particularly those with MEN2B. Parents who do not wish to have prenatal RET gene mutation testing should be offered genetic counseling and informed of the availability of genetic testing of their child to detect a mutated RET allele. This is particularly important for mutations associated with the onset of MTC before 5 years of age." In addition, testing to exclude a pheochromocytoma should be done prior to pregnancy for all women with MEN2. No studies address the effect of pregnancy on MTC progression in patients with biochemical or structural evidence of residual or metastatic disease.

\section{FETAL AND NEONATAL CONSIDERATIONS}

\section{Question 80: What is the relationship between} maternal and fetal thyroid hormone status?

Under normal circumstances the fetal hypothalamicpituitary-thyroid system develops relatively independently of maternal influence because of the presence of the placenta, which regulates the passage of many substances, including $\mathrm{T} 4$, to the fetus. Circulating fetal thyroid hormone levels therefore largely reflect the stage of fetal maturation.

Thyroid hormone receptors are present in the fetal brain at low concentrations up to week 10 of gestation. Thyroid hormone receptor concentrations then rapidly increase 10-fold through week 16 of pregnancy (498).

Serum TT4 and FT4 are first measurable in low levels in fetal serum at 12-14 weeks of development (499-501). 
Based on values obtained by fetal cord sampling in normal pregnant women, it has been estimated that the mean fetal serum TT4 is $2 \mu \mathrm{g} / \mathrm{dL}(26 \mathrm{nmol} / \mathrm{L})$ at 12 weeks, and a relatively larger proportion is in the free form. Beginning in midgestation, the fetal TT4 concentration begins to increase and typically reaches values comparable to nonpregnant females $(10 \mu \mathrm{g} / \mathrm{dL}[138 \mathrm{nmol} / \mathrm{L}])$ by 36 weeks. This rise in TT4 follows an increase in the serum TBG concentration due primarily to the stimulatory effects of maternal estrogen on the fetal liver. In addition, owing to up-regulation of the TSH receptor, there is an increase in the fetal FT4 concentration from a mean of approximately $0.1 \mathrm{ng} / \mathrm{dL}(1.3 \mathrm{pmol} / \mathrm{L})$ to $2 \mathrm{ng} /$ $\mathrm{dL}(25.7 \mathrm{pmol} / \mathrm{L})$, beginning early in the third trimester (502).

In contrast to $\mathrm{T} 4$, the fetal circulating levels of its active metabolite T3 remain significantly lower in fetal life than postnatally, whereas the inactive metabolites reverse T3 and T3 sulfate are elevated. The fetal serum T3 concentration is approximately $6 \mathrm{ng} / \mathrm{dL}(0.09 \mathrm{nmol} / \mathrm{L})$ at 12 weeks, rising to $45 \mathrm{ng} / \mathrm{dL}(0.68 \mathrm{nmol} / \mathrm{L})$ at 36 weeks. Despite the low levels of circulating T3, brain T3 levels are $60 \%-80 \%$ those of the adult by fetal age 20-26 weeks (503). This reflects the importance of local conversion of T4 to T3 in the brain itself. During the second trimester, expression of type 2 deiodinase, which converts $\mathrm{T} 4$ to $\mathrm{T} 3$, increases in the fetal cerebral cortex in parallel to the T3 concentrations (504). T3 sulfate may also serve as an alternate source of T3 for the pituitary (505).

The serum concentration of TSH rises modestly from $\sim 4 \mathrm{mU} / \mathrm{L}$ at 12 weeks to $8 \mathrm{mU} / \mathrm{L}$ at term and is always higher than the corresponding maternal levels $(500,506)$. The reason for this is not completely understood but may be a consequence of incomplete maturation of the hypothalamicpituitary-thyroid axis and/or the high TRH levels in the fetus.

\section{Question 81: What is the role of placental transfer?}

Thyroid hormone. The placenta plays a key role in regulating the exchange of products between the mother and fetus. The amount of placental transfer and the consequent effect on fetal thyroid function varies widely.

Under normal circumstances, the human placenta has modest permeability for T4 because of the predominance of placental deiodinase (D3), which serves to inactivate most of the thyroid hormone presented from the maternal to the fetal circulation. The iodide released by this process can be used as a substrate for fetal thyroid hormone synthesis. The placenta also expresses some D2 (an activating deiodinase), but placental D3 activity is approximately 200-fold greater than D2; the activity of both D2 and D3 falls as pregnancy progresses. The placenta also expresses a range of thyroid hormone transporters, binding proteins, sulfotransferases, and sulfatases. The balance of all of these enzymatic processes determines the amount of T4 transfer that occurs (507).

In the first half of pregnancy, before fetal thyroid maturation, $\mathrm{T} 4$ is detectable in the fetal brain, indicating that mechanisms exist for small but significant transfer of maternal $\mathrm{T} 4$ to the fetal circulation $(503,507)$. This transfer of maternal thyroid hormone is particularly important when the fetus is hypothyroid and probably explains the normal or near-normal cognitive outcomes of babies diagnosed with severe congenital hypothyroidism at birth and treated adequately and sufficiently early following birth. For example, even in infants with congenital absence of thyroid peroxidase
(TPO), the cord serum concentration of T4 is between $25 \%$ and $50 \%$ of normal (508). This maternal T4 disappears rapidly from the newborn circulation, with a half-life of approximately 3-4 days.

lodine. Iodine is actively transported across the placenta from the maternal circulation to the fetus. The placenta actively concentrates iodine through expression of the sodium iodide transporter, the concentration of which increases with gestational age (509).

Immunoglobulin G. Maternal autoantibodies of the immunoglobulin $\mathrm{G}$ ( $\mathrm{IgG}$ ) class are transported across the placenta from approximately midgestation onward by a cellular receptor termed the neonatal Fc receptor, thereby providing the fetus and neonate with humoral immune protection until the infant starts producing its own $\operatorname{IgG}$ (510). In mothers with autoimmune thyroid disease, autoantibodies to TPO, Tg, and the TSH receptor can be transmitted to the fetus in a similar fashion. Whereas TPO antibodies and Tg antibodies do not significantly affect fetal or neonatal thyroid function, antibodies to the TSH receptor can stimulate or block thyroid hormonogenesis or be biologically neutral (511) (see sections VII and VIII on Hypothyroidism and Thyrotoxicosis). Maternal transmission of antibodies to thyroid hormone has also been reported as a cause of congenital hypothyroidism (512).

Hyperthyroidism generally develops only in babies born to mothers with the most potent (or highest concentrations of) stimulatory TRAb activity $(513,514)$. This corresponds to $1 \%-2 \%$ of mothers with GD, or 1 in 50,000 newborns, an incidence that is approximately 4 times higher than that for transient neonatal hypothyroidism due to maternal TSH receptor blocking antibodies (515). Some mothers have mixtures of stimulating and blocking antibodies in their circulation, the relative proportion of which may change over time. Not surprisingly, the clinical picture in the fetus and neonate of these mothers is complex and depends not only on the relative proportion of each activity in the maternal circulation at any one time but on the rate of their clearance from the neonatal circulation postpartum. Thus, one affected mother gave birth, in turn, to a normal infant, a baby with transient hyperthyroidism, and one with transient hypothyroidism (516). In another neonate, the onset of hyperthyroidism did not become apparent until 1-2 months postpartum when the higher-affinity blocking antibodies had been cleared from the neonatal circulation (517). In the latter case, multiple TSH receptor stimulating and blocking antibodies were isolated from maternal peripheral lymphocytes. Each monoclonal antibody recognized different antigenic determinants (epitopes) on the receptor and had different functional properties (518). Occasionally, neonatal hyperthyroidism may occur in infants born to hypothyroid mothers. In these situations, the maternal thyroid has been destroyed by prior radioablation or surgery so that potent thyroid stimulating antibodies, present in the maternal circulation, affect the fetal thyroid but not the maternal thyroid.

$\mathrm{TSH}$ receptor blocking, Ab-induced congenital hypothyroidism, is much less common than neonatal GD but should be suspected in any child with congenital hypothyroidism born to a mother with severe autoimmune thyroid disease, especially if there is a history of a similarly affected sibling (515). Blocking Ab-induced congenital hypothyroidism is 
important to identify because it identifies a child who is likely to have transient disease $(515,519)$. Affected infants may have significantly impaired cognitive outcomes despite early and adequate postnatal treatment if maternal hypothyroidism was present and untreated during gestation (520). The halflife of $\mathrm{IgG}$ is $1-2$ weeks, and the duration of the Ab-induced hypothyroidism is usually about 3 months, but it may be longer.

Significant transplacental transfer of maternal TRH occurs, but not maternal TSH, hCG, or Tg. Importantly, both ATDs (PTU and MMI) as well as TSH receptor antibodies can pass from the mother and the fetus. This may have significant effects on fetal and neonatal thyroid function, as discussed elsewhere.

\section{Question 82: When maternal thyroid abnormalities are detected, how and when should information be provided to the neonatologist or pediatrician?}

\section{Question 83: What information should be provided to the neonatologist or pediatrician?}

Most infants born to women with known thyroid illness are healthy. Furthermore, all newborn infants in the United States are screened for thyroid dysfunction as part of universal screening mandates. Nonetheless, maternal thyroid illness, abnormal maternal thyroid function, the presence of maternal TSH receptor antibodies, and/or the use of antithyroid medications during gestation can each contribute to adverse effects in the newborn. Therefore, knowledge of these facts is important and should be documented in the newborn infant's medical record. In many cases, direct communication with the neonatologist or pediatrician is recommended, especially in the setting of severe maternal hyperthyroidism or maternal use of ATDs at any time throughout pregnancy. The etiology, timing, severity, and treatment of maternal thyroid disease should all be conveyed. In women with GD (even if previously ablated with ${ }^{131}$ I or athyreotic following surgery) concentrations of circulating TRAb should be assessed at initial thyroid function testing in early pregnancy, and if TRAb is positive, again at weeks 18-22 (see Section VIII). Such information should be documented and conveyed to the pediatrician because maternally derived thyroid antibodies as well as antithyroid medication can transfer to the fetus and affect newborn thyroid health.

\section{- RECOMMENDATION 71}

A history of maternal thyroid illness, use of antithyroid medications (PTU, MMI) during gestation, or measurements of abnormal maternal thyroid function or TRAb during gestation should be communicated to the newborn's neonatologist or pediatrician.

Strong recommendation, moderate-quality evidence.

\section{- RECOMMENDATION 72}

The severity of maternal and fetal thyroid illness should guide the timing of communication. Severe, progressive, or complex thyroid illness during pregnancy mandates communication with the neonatologist or pediatrician before birth and consideration of consultation with a pedi- atric endocrinologist. Most other illness is optimally communicated shortly after birth.

Strong recommendation, moderate-quality evidence.

\section{Question 84: Should all neonates be screened for thyroid dysfunction?}

Congenital hypothyroidism is one of the most frequent treatable causes of intellectual impairment, and newborn screening for the detection of congenital hypothyroidism is performed routinely in all 50 states of the United States, as well as in Canada, Europe, Israel, Japan, Australia, and New Zealand (521). Screening programs are also being introduced in many developing countries, accounting overall for approximately one third of the world's population (521). Blood spot specimens eluted from filter paper are utilized. The optimal timing of measurement is at $2-5$ days of age in order to avoid confounding by the physiologic surge in neonatal TSH, which occurs shortly after birth. Some programs employ primary T4 screening with TSH measurement when specimens demonstrate a value below a specified cutoff. Others use a primary TSH determination with reflex T4 strategy. The introduction of newborn screening for congenital hypothyroidism has led to the virtual elimination of intellectual impairment due to hypothyroidism so long as early and adequate postnatal LT4 treatment is initiated immediately $(522,523)$.

\section{- RECOMMENDATION 73}

All newborns should be screened for hypothyroidism by blood spot analysis typically 2-5 days after birth.

Strong recommendation, high-quality evidence.

\section{Question 85: How is neonatal hypothyroidism treated?}

In women receiving ATDs at the time of delivery, transplacental transfer of the medication can potentially induce neonatal hypothyroidism. In such cases, neonatal metabolism removes the remaining MMI or PTU from the newborn circulation. This results in the return of normal thyroid function, typically within 3-5 days. For neonates with congenital hypothyroidism, LT4 must be administered. The recommended starting dose for full-term infants is $10-15 \mu \mathrm{g} / \mathrm{kg} / \mathrm{d}$, and depends on the severity of the initial hypothyroidism $(522,523)$. In premature hypothyroid infants a lower dose is generally utilized. For optimal cognitive outcomes, therapy should be initiated within 2 weeks of life. Both inadequate and excess thyroid hormone replacement may be harmful, so close follow-up is important, particularly during the first 3 years of life. During this time, brain development is thyroid hormone dependent. Age-dependent normative values for TSH and T4 should be used to guide therapy. Until further information becomes available, infants with severe congenital hypothyroidism (i.e., initial serum $\mathrm{TSH}$ concentration $>100 \mathrm{mU} / \mathrm{L}$ ) should remain on a single formulation of LT4 without substitution (524). Treatment should be guided by a pediatric endocrinologist or experienced pediatrician.

The presence of a goiter in a newborn should prompt referral to a pediatric endocrinologist. An ultrasound examination should be performed to evaluate the patency of the trachea. A goiter may reflect a hypothyroid or hyperthyroid 
state in response to maternal thyroid illness or overtreatment with antithyroid medications. Regardless, close observation and urgent treatment may be required to achieve a euthyroid state and avoid airway compromise in the newborn.

For a more detailed discussion of this and other aspects of neonatal hypothyroidism, the reader is referred to several recent excellent reviews and guidelines $(522,523,525)$.

\section{Question 86: How is neonatal hyperthyroidism treated?}

Most neonatal hyperthyroidism is caused by maternal transfer of TRAb to the fetus. Typically, neonatal GD does not present until the end of the first week of life when maternal ATD, but not the TRAb, have been cleared from the neonatal circulation. This may be delayed in babies born to mothers with a mixture of stimulating and blocking antibodies. Thus, results on newborn screening may be paradoxically normal. A high degree of suspicion for infants at risk is important.

The conventional treatment for neonatal hyperthyroidism is PTU $5-10 \mathrm{mg} / \mathrm{kg}$ administered in divided doses. However recent evidence of rare hepatotoxicity due to this agent has led to a switch to MMI therapy $(0.5-1 \mathrm{mg} / \mathrm{d})$ by many pediatric endocrinologists. Propranolol $(2 \mathrm{mg} / \mathrm{kg})$ may be added if the hyperthyroidism is severe. Close follow-up of the affected newborn is important, with downward adjustment in the dose of ATD required as the hyperthyroidism resolves. The usual duration of neonatal GD is 1-3 months, but it depends on antibody potency. Separate from neonatal GD (which is self-limited), neonatal hyperthyroidism may rarely be caused by a gain-of-function mutation in the TSH receptor, or by McCune-Albright syndrome. In such cases, newborn hyperthyroidism may be permanent (526).

\section{THYROID DISEASE AND LACTATION}

\section{Question 87: Does maternal thyroid hormone status impact lactation?}

\section{Question 88: In the breastfeeding mother, should maternal hypothyroidism be treated to improve lactation?}

\section{Question 89: In the breastfeeding mother, should maternal hyperthyroidism be treated to improve lactation?}

Abnormal maternal thyroid hormone concentrations (both hypothyroidism and hyperthyroidism) can impact milk letdown and the ability to successfully breastfeed. While there are no controlled or randomized human trials, several case series and cohort analyses have been reported. One study reported 26 human pregnancies complicated by hypothyroidism in New Delhi, India. Deficient lactation was reported in $19.2 \%$ of subjects (527). Other case reports describe impaired lactation, which improved upon successful treatment of hypothyroidism (528). Interventional trials in nonhuman mammals corroborate these findings. Hapon and colleagues (529) investigated the effects of PTU-induced hypothyroidism in rats at days 10-12 of lactation. After 30 minutes of suckling, milk excretion was reduced compared to euthyroid controls. The authors suggested mammary function was maintained while a reduced milk ejection reflex was likely responsible. A separate investigation of Brahman cows demonstrated reduced milk production in PTU-induced hypothyroid cows compared to euthyroid controls. Following cessation of PTU and normalization of thyroid status, lactation in these animals returned to baseline (530). Together, these data strongly suggest maternal hypothyroidism can adversely affect lactation. However, with limited human data and no controlled or randomized intervention studies, it is unclear what proportion of hypothyroid mothers would be affected. Furthermore, it is possible that many mothers experiencing only mild to moderate hypothyroidism could experience only minimal difficulties with breastfeeding and milk production. Importantly, the available data confirm that maternal hypothyroidism itself is not harmful to the breastfeeding infant per se, so long as adequate nutrition (milk) is provided. Therefore, it appears most reasonable to recommend evaluation of thyroid function in women who demonstrate poor lactation, with the goal of effectively returning any hypothyroid mother to a euthyroid state.

Maternal hyperthyroidism may also impair lactation, although the data are less clear. No controlled human trial or large cohort analysis has been performed. Two rat studies demonstrated adverse effects of hyperthyroidism on lactation. In one study, moderate to severe hyperthyroidism was induced in pregnant rats during and after pregnancy. Complete lactation failure was noted (531). A second study in which moderate hyperthyroidism was induced demonstrated continued ability of rats to lactate, but impairment of milk ejection. Ultimately, this resulted in death of the offspring due to malnutrition (532). Such limited data, obtained from only nonhuman populations, make it impossible to draw firm conclusions. Therefore, no recommendation can be made to treat maternal hyperthyroidism on the grounds that it will improve lactation.

\section{- RECOMMENDATION 74}

As maternal hypothyroidism can adversely impact lactation, women experiencing poor lactation without other identified causes should have TSH measured to assess for thyroid dysfunction.

Weak recommendation, low-quality evidence.

\section{- RECOMMENDATION 75}

Given its adverse impact upon milk production and letdown, subclinical and overt hypothyroidism should be treated in lactating women seeking to breastfeed.

Weak recommendation, low-quality evidence.

\section{- RECOMMENDATION 76}

The impact of maternal hyperthyroidism upon lactation is not well understood. Therefore, no recommendation to treat maternal hyperthyroidism on the grounds of improving lactation can be made at this time.

No recommendation, insufficient evidence.

\section{Question 90: Is maternal thyroid hormone (thyroxine or LT4) transferred to the newborn via breast milk?}

A small amount of maternal thyroid hormone is present in the breast milk of lactating women, and also detected in cow, 
goat, and sheep milk. A study investigated T4 and T3 in the breast milk of mothers who delivered very premature infants. Thyroxine concentration in breast milk ranged from 0.17 to $1.83 \mu \mathrm{g} / \mathrm{L}$ (mean $0.83 \mu \mathrm{g} / \mathrm{L}$ ) (533). Assuming an average consumption of $\sim 750 \mathrm{~mL} / \mathrm{d}$ of breast milk, this equates to approximately $0.62 \mu \mathrm{g}$ of T4 per day. Assuming total T4 requirements of $40-60 \mu \mathrm{g} / \mathrm{d}$ for most athyreotic infants, the amount of thyroxine transferred to the infant via the human lactating breast is $\sim 1 \%$ of the total daily requirement. Thus, maternal transfer of thyroid hormone does not have a meaningful impact on the infant's thyroid hormone status.

Studies in cows (534), sheep (535), and donkeys (536) similarly find a small but detectable concentration of $\mathrm{T} 4$ and T3 in the milk supply. One study demonstrated secretion of $200-300 \mathrm{pg} / \mathrm{mL}$ of T3 in cow milk. This translates into a total daily consumption of $0.2-0.4 \mu \mathrm{g} / \mathrm{d}$ depending the age and feeding habits of the infant. Similarly, T4 concentrations in cows' milk demonstrate an average concentration of $0.97 \mathrm{ng} /$ $\mathrm{mL}$, translating into the consumption of approximately 0.75 $1.5 \mu \mathrm{g}$ T4 per day. Thus, dairy milk obtained from cows, sheep, goats, or donkeys has little impact on the thyroid health of the infant or young child.

\section{Question 91: Should diagnostic or therapeutic radiopharmaceuticals be administered to breastfeeding or lactating women?}

Because radioactive iodine is concentrated in breast milk, and the half-life of ${ }^{131} \mathrm{I}$ is relatively long ( $\sim 8$ days), use of ${ }^{131}$ I for scanning or treatment is absolutely contraindicated in the breastfeeding mother. The half-life of ${ }^{123} \mathrm{I}$ is 13 hours. If circumstances necessitate the use of ${ }^{123}$ I for of thyroid uptake measurement or scans while lactating, breast milk should be pumped and discarded for several days until the radioactive iodine has cleared from the body (537).

\section{- RECOMMENDATION 77}

The use of ${ }^{131} \mathrm{I}$ is contraindicated during lactation. If required, ${ }^{123}$ I can be used if breast milk is pumped and discarded for 34 days before breastfeeding is resumed. Similarly, Tc-99m pertechnetate administration requires breast milk to be pumped and discarded during the day of testing.

Strong recommendation, moderate-quality evidence.

\section{Question 92: Are ATDs (PTU, MMI) transferred into breast milk, and what are the clinical consequences to the breastfed infant?}

Both PTU and MMI can be detected in the breast milk of treated hyperthyroid women. This finding raised initial concern that consumption of these medications could prove detrimental to the health of the breastfeeding infant. However, studies first performed using PTU confirmed that only a very small amount of the drug is transferred from maternal serum into breast milk. In a study of nine women given $200 \mathrm{mg}$ PTU orally, milk PTU concentration was measured for 4 hours thereafter and only $0.007 \%-0.077 \%$ of the ingested dose was detected. The authors calculated that a lactating mother consuming PTU $200 \mathrm{mg}$ three times daily would transmit only $149 \mu \mathrm{g}(0.149 \mathrm{mg})$ of PTU daily to her infant (538). This is well below a therapeutic dose and deemed to pose no risk to the breastfeeding infant. Separate studies confirm normal thyroid function in breastfed infants of mothers consuming PTU $(539,540)$. In these studies, two infants were found to have an elevated TSH within 1 week of birth, however, these values normalized thereafter despite continued breastfeeding. This suggests the cause of neonatal hypothyroidism in these infants was transplacental passage of PTU before birth, as opposed to any adverse effect of PTU transferred via breast milk. The remaining children who consumed breast milk expressed from mothers taking PTU had normal thyroid function.

Studies of MMI or CM, confirm a 4- to 7-fold higher proportion of the medication transferred into maternal milk in comparison to PTU. Approximately $0.1 \%-0.2 \%$ of an orally administered $\mathrm{MMI} / \mathrm{CM}$ dose is excreted into breast milk $(541,542)$. Johansen and colleagues (541) calculated that a single $40 \mathrm{mg}$ dose of MMI could result in delivery of $70 \mu \mathrm{g}$ $(0.07 \mathrm{mg})$ to the breastfeeding infant. Several studies have separately investigated the effect of maternally ingested MMI or CM upon the thyroid status of the breastfeeding infant (543-545). Virtually all have documented normal neonatal thyroid function. Furthermore, several women overtreated with $\mathrm{MMI} / \mathrm{CM}$ were found to have elevated TSH concentrations (TSH 19-102 mU/L). Even in these situations, normal neonatal thyroid function was nonetheless confirmed in breastfeeding infants.

The largest study investigating the effects of maternal MMI consumption during lactation was performed by Azizi and colleagues (545). Importantly, this study assessed both neonatal thyroid function in the breastfeeding offspring, but also intellectual development and physical growth in a subset of infants. Verbal and performance IQ scores were measured in 14 children who breastfed from MMI-treated mothers, with comparison to 17 control children. Testing was performed between 48 and 74 months of age. No difference was detected in the IQ or physical development of the breastfeeding children compared to the control children (543).

Together, these data have led experts to confirm the safety of low to moderate doses of both PTU and MMI/CM in breastfeeding infants. However, given the relatively small size of the studied population, maximal daily doses of $20 \mathrm{mg}$ MMI or $450 \mathrm{mg}$ PTU are advised (352).

\section{Question 93: What is the approach to the medical treatment of maternal hyperthyroidism in lactating women?}

\section{- RECOMMENDATION 78}

Excepting treatment decisions specifically made on the grounds of improving lactation (discussed above), the decision to treat hyperthyroidism in lactating women should be guided by the same principles applied to nonlactating women.

Strong recommendation, low-quality evidence.

\section{Question 94: When medical treatment of maternal hyperthyroidism is indicated, what medications should be administered?}

\section{- RECOMMENDATION 79}

When antithyroid medication is indicated for women who are lactating, both MMI (up to maximal dose of $20 \mathrm{mg} / \mathrm{d}$ ) 
and PTU (up to maximal dose of $450 \mathrm{mg} / \mathrm{d}$ ) can be administered. Given a small, but detectable amount of both PTU and MMI transferred into breast milk, the lowest effective does of MMI/CM or PTU should always be administered.

\section{Strong recommendation, moderate-quality evidence.}

\section{Question 95: How should breastfeeding children of mothers who are treated with antithyroid medications be monitored?}

\section{- RECOMMENDATION 80}

Breastfed children of women who are treated with ATDs should be monitored for appropriate growth and development during routine pediatric health and wellness evaluations. Routine assessment of serum thyroid function in the child is not recommended.

Weak recommendation, moderate-quality evidence.

\section{Question 96: What are the iodine nutritional considerations in lactating women?}

Iodine is an essential nutrient required for thyroid hormone production and is primarily derived from the diet. For most breastfeeding infants, breast milk is the sole (or primary) source of nutrition and thus of dietary iodine. Therefore, adequate iodine intake in the lactating mother positively affects infant health. Iodine requirements increase during pregnancy to accommodate the maternal-fetal unit. Post partum, maternal iodine requirements increase even further, as iodine is transferred to the breastfeeding infant. The dietary goals for individual total daily iodine intake (dietary and supplement) recommended by the Institute of Medicine are $290 \mu \mathrm{g} / \mathrm{d}$ for women who are breastfeeding (546). The WHO recommends $250 \mu \mathrm{g} / \mathrm{d}$ for both pregnant women and lactating women (547).

As discussed in Section IV, dietary sources of iodine vary regionally, and the dietary iodine intake of individuals cannot be reliably ascertained either by patient history or by any laboratory measure. Owing to concerns that a portion of women in the United States are mildly to moderately iodine deficient (which can be exacerbated during lactation due to increased demand), iodine supplementation for breastfeeding mothers is recommended. The ATA has previously recommended $150 \mu \mathrm{g} / \mathrm{d}$ as iodine supplementation for all North American women who are breastfeeding (117). The goal of supplementation is to augment rather than replace dietary iodine intake.

Unfortunately, recommendations for iodine supplementation when breastfeeding have not seen widespread adoption. In the NHANES 2001-2006 survey, only 15\% of lactating women reported ingesting iodine-containing supplements (121). Furthermore, one analysis confirmed that only $51 \%$ of U.S. prenatal vitamins contained iodine (122). Therefore, physician counseling at the time of postbirth hospital discharge is paramount.

\section{- RECOMMENDATION 81}

All breastfeeding women should ingest approximately $250 \mu \mathrm{g}$ of dietary iodine daily.

Strong recommendation, high-quality evidence.

\section{- RECOMMENDATION 82}

Breastfeeding women should supplement their diet with a daily oral supplement that contains $150 \mu \mathrm{g}$ of iodine. This is optimally delivered in the form of potassium iodide (present in a multivitamin) because kelp and other forms of seaweed do not provide a consistent delivery of daily iodine.

Strong recommendation, moderate-quality evidence.

\section{- RECOMMENDATION 83}

In severely iodine-deficient, low-resource regions, where universal salt iodization is lacking and daily supplementation is not feasible, lactating women should receive one dose of $400 \mathrm{mg}$ iodine as oral iodized oil soon after delivery.

\section{Strong recommendation, high-quality evidence.}

\section{- RECOMMENDATION 84}

As is the case during pregnancy, sustained iodine intake while breastfeeding that exceeds $500-1100 \mu \mathrm{g} / \mathrm{d}$ should be avoided because of concerns about the potential for inducing hypothyroidism in the infant.

Strong recommendation, moderate-quality evidence.

\section{POSTPARTUM THYROIDITIS}

\section{Question 97: What is the definition of PPT and what are its clinical implications?}

Postpartum thyroiditis is the occurrence of thyroid dysfunction, excluding GD, in the first postpartum year in women who were euthyroid prior to pregnancy (548). This is an inflammatory autoimmune condition. In the classic form, transient thyrotoxicosis is followed by transient hypothyroidism with a return to the euthyroid state by the end of the initial postpartum year (2). The clinical course of PPT varies, with approximately one quarter of patients presenting with the classical form, one quarter with isolated thyrotoxicosis, and one half presenting with isolated hypothyroidism (431). The thyrotoxic phase of PPT typically occurs between 2 and 6 months post partum, but episodes have been reported as late as 1 year following delivery. All episodes of thyrotoxicosis resolve spontaneously. The hypothyroid phase of PPT occurs from 3 to 12 months postpartum with $10 \%-20 \%$ of cases resulting in permanent hypothyroidism. It should be noted, however, that a prospective study reported that $50 \%$ of women with PPT remained hypothyroid at the end of the first postpartum year (549).

\section{Question 98: What is the etiology of PPT?}

Postpartum thyroiditis is an autoimmune disorder associated with the presence of thyroid antibodies (TPOAb and $\operatorname{Tg} \mathrm{Ab}$ ), lymphocyte abnormalities, complement activation, increased levels of IgG1, increased NK cell activity, and specific HLA haplotypes (550-552). Women who are thyroid $\mathrm{Ab}$ positive in the first trimester have a high risk of developing PPT, ranging from $33 \%$ to $50 \%$ (553). Women with the highest $\mathrm{Ab}$ titers also have the highest risk of PPT (431). The occurrence of PPT reflects the rebound of the immune system in the postpartum period after the relative immune suppression of pregnancy. 
Question 99: How should the etiology of new thyrotoxicosis be determined in the postpartum period?

The major challenge is to differentiate thyrotoxicosis caused by PPT from thyrotoxicosis caused by GD. This is an important distinction because the two disease entities require different treatments and have markedly different clinical courses. The timing of onset provides some clues about etiology. In a Japanese hospital study of 42 patients who developed thyrotoxicosis within the first year after pregnancy, $86 \%$ of patients who had debut of disease within the first 3 months after delivery had thyroiditis, whereas all who developed thyrotoxicosis after 6.5 months had GD (554).

$\mathrm{TSH}$ receptor antibodies are positive in GD in nearly all cases and are typically negative in PPT, although some mixed-type disease is seen. An elevated T4:T3 ratio suggests the presence of PPT. Physical stigmata of GD, such as goiter with a bruit or ophthalmopathy, are diagnostic when present. The radioiodine uptake is elevated or normal in GD and low in the thyrotoxic phase of PPT, but the use of radioactive diagnostic procedures in lactating patients is rarely indicated. Due to their short half-lives, ${ }^{123}$ I or technetium scans (Tc-99m) may be used in women who are breastfeeding if breast milk is pumped and discarded for several days after the scan pending the isotope used (see Section XI). As described in Section XI, the use of ${ }^{131} \mathrm{I}$ is contraindicated in women who are breastfeeding.

\section{Question 100: What is the prevalence of PPT?}

The prevalence of PPT is approximately $5 \%$, although it has varied markedly in different studies, with frequencies reported from $1.1 \%$ to $16.7 \%$ (555). Women with other autoimmune disorders have an increased risk of PPT. Specifically, the prevalence of PPT is 3-4 times higher in women with diabetes mellitus type 1 compared to unselected populations $(556,557)$. The frequency of PPT is $25 \%$ in women with chronic viral hepatitis (558), $14 \%$ in women with systemic lupus erythematosus (559), $44 \%$ in women with a prior history of GD (560), and $27 \%$ in patients with antipituitary antibodies (561). Individuals who recover fully from PPT have a $70 \%$ chance of developing PPT in each subsequent pregnancy (562). Women on LT4 therapy secondary to Hashimoto's thyroiditis may develop PPT if their thyroid gland is not completely atrophic (563). Cases of PPT have been reported following pregnancy loss, but the prevalence of PPT following pregnancy loss is unknown (564).

\section{Question 101: What symptoms are associated with PPT?}

Postpartum thyroiditis is a painless condition and most women are asymptomatic or only mildly symptomatic during the thyrotoxic phase. This is because the degree of increase in thyroid hormones is typically mild, and T4 levels are usually more elevated than T3. Nevertheless, in prospective studies, reported symptoms include irritability, heat intolerance, fatigue, and palpitations (431,565-567). The hypothyroid phase of PPT is more frequently symptomatic. Symptoms during the hypothyroid phase of PPT may include cold intolerance, dry skin, fatigue, impaired concentration, and paresthesias $(566,567)$. One study demonstrated that patients with TPO antibodies and PPT had more symptoms than those who were TPOAb negative (568).

\section{Question 102: Is PPT associated with depression?}

Studies evaluating the relationship of PPT to postpartum depression have been inconsistent (569-573). Two studies have reported a significant association between the presence of thyroid antibodies and depression $(568,574)$, irrespective of thyroid function, whereas another study showed no association between the presence of microsomal antibodies and postpartum depression (575). A prospective trial of LT4 treatment versus placebo in postpartum TPOAb-positive women resulted in no difference in rates of postpartum depression between the two groups (576).

\section{- RECOMMENDATION 85}

All patients with depression, including postpartum depression, should be screened for thyroid dysfunction.

Strong recommendation, low-quality evidence.

\section{Question 103: What is the treatment for the thyrotoxic phase of PPT?}

There have been no prospective studies evaluating when and how to treat PPT. Treatment of the thyrotoxic phase is guided by its transitory nature. ATDs (PTU and MMI) are ineffective in treating the thyrotoxic phase of PPT because it is a destructive thyroiditis in which thyroid hormone synthesis is not increased. Symptoms are typically mild. In rare cases when symptoms are clinically significant, propranolol at the lowest possible dose to alleviate symptoms may be used. The thyrotoxic phase of PPT must be differentiated from recurrent or de novo GD.

\section{- RECOMMENDATION 86}

During the thyrotoxic phase of PPT, symptomatic women may be treated with $\beta$-blockers. A $\beta$-blocker that is safe for lactating women, such as propranolol or metoprolol, at the lowest possible dose to alleviate symptoms is the treatment of choice. Therapy is typically required for a few weeks.

Strong recommendation, moderate-quality evidence.

\section{- RECOMMENDATION 87}

ATDs are not recommended for the treatment of the thyrotoxic phase of PPT.

Strong recommendation, high-quality evidence.

\section{Question 104: Once the thyrotoxic phase of PPT resolves, how often should TSH be measured to screen for the hypothyroid phase?}

\section{- RECOMMENDATION 88}

Following the resolution of the thyrotoxic phase of PPT, serum TSH should be measured in approximately 4-8 weeks (or if new symptoms develop) to screen for the hypothyroid phase.

Strong recommendation, high-quality evidence. 


\section{Question 105: What is the treatment for the hypothyroid phase of PPT?}

In women with significant symptoms, those currently lactating or women who are actively attempting pregnancy, treatment should be started (431). LT4 treatment should be considered during the hypothyroid phase of PPT if the patient is mildly symptomatic, or recommended if the patient is considering another conception. If treatment is delayed, thyroid function should be rechecked every 4-8 weeks until euthyroidism is restored and women should be counseled to use contraception.

\section{- RECOMMENDATION 89}

LT4 should be considered for women with symptomatic hypothyroidism due to PPT. If treatment is not initiated, their TSH level should be checked every 4-8 weeks until thyroid function normalizes. LT4 should also be started in hypothyroid women who are attempting pregnancy or who are breastfeeding.

Weak recommendation, moderate-quality evidence.

\section{Question 106: How long should LT4 be continued once initiated?}

The length of time that LT4 should be continued has not been systematically evaluated. Guiding principles are to maintain a euthyroid state in women who are attempting pregnancy or pregnant. Tapering LT4 doses in order to determine whether the hypothyroid phase of PPT was transitory or permanent can begin by 12 months post partum. Tapering should be gradual and TSH should be monitored every 6-8 weeks.

\section{- RECOMMENDATION 90}

If LT4 is initiated for PPT, discontinuation of therapy should be attempted after 12 months. Tapering of LT4 should be avoided when a woman is actively attempting pregnancy or is pregnant.

Weak recommendation, low-quality evidence.

\section{Question 107: How often should thyroid function testing be performed after the hypothyroid phase of PPT resolves?}

The impact of PPT on long-term thyroid function has been evaluated in seven studies $(549,562,577-581)$. These data demonstrate that $10 \%-50 \%$ of women in whom the hypothyroid phase of PPT initially resolves will ultimately go on to develop permanent hypothyroidism. Factors associated with an increased risk of developing permanent hypothyroidism are multiparity, thyroid hypoechogenicity on ultrasound, greater severity of the initial hypothyroidism, higher $\mathrm{TPOAb}$ titers, greater maternal age, and a history of pregnancy loss.

\section{- RECOMMENDATION 91}

Women with a prior history of PPT should have TSH testing annually to evaluate for the development of permanent hypothyroidism.

Strong recommendation, high-quality evidence.

\section{Question 108: Does treatment of thyroid Ab-positive euthyroid women during pregnancy prevent PPT?}

Two randomized placebo-controlled controlled trials have evaluated the efficacy of iodine or LT4 treatment during pregnancy to prevent the development of PPT in thyroid Abpositive women. Neither intervention decreased the incidence of PPT $(154,582)$. A single trial has suggested a benefit of selenium supplementation for the prevention of PPT in TPOAb-positive pregnant women, though this has not been independently validated. Furthermore, selenium use has been associated with an increased risk of type 2 diabetes (156). Currently there is not sufficient evidence to recommend selenium supplementation during pregnancy in thyroid $\mathrm{Ab}-$ positive women (see Recommendation 12).

\section{- RECOMMENDATION 92}

Treatment of euthyroid thyroid Ab-positive pregnant woman with either LT4 or iodine to prevent PPT is ineffective and is not recommended.

\section{Strong recommendation, high-quality evidence.}

\section{SCREENING FOR THYROID DYSFUNCTION BEFORE OR DURING PREGNANCY}

Whether to universally screen for thyroid disease either before or during pregnancy remains controversial. For universal screening to be recommended, any index condition must be prevalent, associated with adverse health outcomes, and treatable. Furthermore, effective therapy must exist but also be practical and effectively deliverable. Finally, screening must be cost effective.

Screening for thyroid dysfunction in pregnancy fulfills some of these criteria. Hypothyroidism, hyperthyroidism, and thyroid autoimmunity are common conditions, with prevalence rates of $2 \%-3 \%(259,260,289,583), 0.1 \%-0.4 \%$ (397), and up to $17 \%$ (137), respectively. Untreated overt hypothyroidism and hyperthyroidism are associated with adverse obstetrical and fetal outcomes. Among overtly hypothyroid, but undiagnosed pregnant women, the majority will remain hypothyroid following pregnancy, with a mean time to diagnosis in one study of 5 years (276). However, overt thyroid dysfunction is less common than subclinical dysfunction and is frequently identified by clinical assessment at the time of presentation. Mild TSH suppression can be seen in normal early pregnancy and, even when TSH is $\leq 2.5 \%$ of the gestational reference range in association with normal FT4 (cutoff between 0.008 and $0.668 \mathrm{mU} / \mathrm{L}$, depending on the gestational age), it is not associated with adverse obstetrical outcomes (22). Thus, the most notable impact of a universal screening mandate for thyroid dysfunction would be the identification of the large proportion of patients with subclinical hypothyroidism (mild elevations in serum TSH with normal thyroid hormone levels).

Presently, most studies investigating subclinical hypothyroidism suggest an association with adverse obstetrical outcomes that is linear because greater degrees of TSH elevation are associated with increased risks to the pregnancy. Such adverse outcomes also appear to be influenced by concomitant antithyroid autoimmunity. Exemplifying this, a large prospective study of 3315 women demonstrated the additive 
effect of anti-TPO positivity to the degree of TSH elevation. In anti-TPO-positive women the risk of pregnancy loss increased significantly beyond a TSH concentration $>2.5 \mathrm{mU} / \mathrm{L}$ (OR 4.95 for TSH 2.5-5.2), with an even greater increase when TSH was $>5.2 \mathrm{mU} / \mathrm{L}$ (OR 9.56 for TSH 5.2-10 mU/L), whereas in antiTPO-negative women, significant increases in risk of pregnancy loss was identified only when TSH concentrations exceeded 5.2 mU/L (OR 3.4 for TSH 5.2-10) (288).

Thyroid status can be accurately assessed with currently available blood tests, including TSH, TT4/FT4, and TPOAb. These tests are relatively inexpensive and widely available. Thus, the principal complexity surrounding the screening question relates to the evidence for treatment effectiveness, especially in the population of pregnant women with subclinical hypothyroidism. While prior retrospective studies have suggested a benefit of LT4 treatment in this population $(276,277)$, such findings have not been sufficiently replicated in blinded, prospective analyses thus far.

Important to this discussion is the understanding of two different adverse endpoints that must be considered. These endpoints are maternal/pregnancy risk (primarily pregnancy loss), and separately the adverse effects on fetal neurocognitive development as manifested by lower IQ in the offspring. These endpoints do not necessarily cosegregate. Therefore, each must be considered separately, as different interventions and their timing may affect such endpoints discordantly.

\section{Question 109: Does identification and LT4 treatment of women with subclinical hypothyroidism or thyroid autoimmunity reduce pregnancy complications and pregnancy loss?}

Retrospective studies have frequently suggested a positive correlation between treatment of mild elevations of maternal serum TSH and decreased risk of pregnancy loss. For example, a retrospective study from Belgium assessed the effect of TSH screening in a small cohort of pregnant women. LT4 treatment of TPOAb-positive women with TSH $>1 \mathrm{mU} / \mathrm{L}$ led to a reduction of pregnancy loss rates from $16 \%$ to $0 \%$ (180). A separate historical cohort analysis studied $>1000$ pregnant women in the United Kingdom treated with long-term LT4. Pregnancy loss rates were significantly higher when TSH was $>4.5 \mathrm{mU} / \mathrm{L}$ (and especially when $>10 \mathrm{mU} / \mathrm{L}$ ) compared with when TSH was $<2.5 \mathrm{mU} / \mathrm{L}$ (318). The retrospective design and lack of TPOAb data are two important limitations of this study.

Limited RCT data on the effects of treatment of subclinical hypothyroidism in pregnancy are available to date. Negro and colleagues (28) randomized euthyroid (TSH $<4.2 \mathrm{mU} / \mathrm{L}$ ) TPOAb-positive women to treatment with LT4 or no treatment. This intervention demonstrated a significant reduction in preterm deliveries (from $22 \%$ to $7 \%$ ) and miscarriage rates (from $14 \%$ to $3.5 \%$ ). However, limitations of this study include its small sample size and the fact that average time of LT4 initiation was late in the first trimester (mean 10.3 weeks), at a time when many pregnancy losses had already occurred.

Two more recent prospective RCTs have investigated the benefits of universal screening of pregnant women with subsequent LT4 treatment of women with TSH $>2.5 \mathrm{mU} / \mathrm{L}$. Negro and colleagues (584) randomized 4562 women to universal screening versus screening of women at high risk for thyroid disease. Women identified to have TPOAb posi- tivity and TSH concentrations $>2.5 \mathrm{mU} / \mathrm{L}$ were treated with LT4. The timing of treatment initiation was 8.8 weeks gestation. Even though this is well into the first trimester, limiting assessment of the outcome, this is the earliest randomized intervention trial published to date. In the primary analysis, there was no significant difference in adverse events detected between the universally screened cohort versus those who underwent high-risk screening. The main limitation of this study was its design given that all high-risk patients were treated in both study arms, limiting the ability to detect a treatment effect. However, in a secondary analysis of the low-risk cohort, a significant decrease in adverse obstetrical and neonatal outcomes was detected when a screening and treatment strategy was applied (OR 0.43 [CI 0.26-0.70]). How to best interpret this finding, however, remains unclear because this study used a composite endpoint for assessing adverse outcomes. For these reasons, this study alone is often viewed as insufficient to warrant adoption of a universal screening mandate.

A second investigation employed cluster randomization at two centers in China, comparing a process of universal screening at one hospital (and treatment when TSH $>2.5 \mathrm{mU} /$ L) to a traditional nonscreening approach in the second institution. In the latter group, serum was obtained from women in early pregnancy, but banked and not analyzed until the postnatal period. The authors concluded that identifying and treating elevated TSH values early in pregnancy reduced pregnancy loss rates (OR 0.343 [CI 0.21-0.56]) and macrosomia (OR 0.46 [CI 0.28-0.74]) (585). However, these data should be viewed with caution because the timing of enrollment at the two centers varied substantially, likely influencing the reporting of pregnancy loss rates. Notably, the screening arm enrolled women at week 11 of pregnancy, whereas the control arm enrolled women at week 7. Because most pregnancy losses occur during the first trimester, the earlier time at testing in the control group suggests that the much higher rates of pregnancy loss were simply due to early enrollment bias.

Importantly, two additional prospective, randomized trials investigating obstetric outcomes are currently in progress. The Thyroid Antibodies and Levothyroxine Trial (586) is a large RCT conducted in the United Kingdom, randomizing TPOAb-positive euthyroid women with a history of pregnancy loss or infertility to treatment with $50 \mu \mathrm{g} \mathrm{LT} 4$ versus placebo. The endpoint is successful delivery of the infant beyond 34 weeks of gestation. Separately, the T4Life trial in the Netherlands is randomizing euthyroid anti-TPOAbpositive women with a history of recurrent pregnancy losses to treatment with LT4 (starting before conception) versus placebo. The primary outcome is live birth after 24 weeks gestation (587).

\section{Question 110: Does identification and LT4 treatment of pregnant women with subclinical hypothyroidism improve neurocognitive outcomes in offspring?}

With regards to the endpoint of fetal neurocognitive development, there are mixed findings from currently available studies. Haddow and colleagues (276) first published seminal data from a large case-control study investigating the effects of untreated maternal hypothyroidism on the IQ of offspring tested at $\sim 8$ years. This study analyzed sera from 25,216 
women obtained at an average gestational age of 17 weeks. They identified 62 women with either TSH concentrations $\geq 99$.7th percentile, or women with TSH concentrations between the 98 and 99.6th percentiles who also had low FT4 concentrations. The authors compared the IQ of the offspring (at 7-9 years) with that of 124 matched children born to euthyroid mothers. IQ scores were 7 points lower in children born to untreated hypothyroid mothers in comparison to controls. However, the retrospective nature of this study has led to questions regarding its reproducibility.

The largest prospective randomized trial of LT4 therapy investigating the treatment of maternal hypothyroidism or hypothyroxinemia in early pregnancy is the CATS study. This study randomized 22,000 women with singleton pregnancies at $<16$ weeks gestation to immediate thyroid function testing and LT4 treatment if serum TSH was $>97.5$ th percentile and/or if serum FT4 was $<2.5$ th percentile. The control arm received no treatment, though serum was obtained in early pregnancy and banked until testing was performed postpartum. The primary outcome was offspring IQ at 3 years of age. Ultimately, the trial found no significant difference in IQ between the children of 390 treated mothers compared with those of 404 untreated mothers (306). Limitations of this study include the timing of LT4 administration (median initiation $>13$ weeks), and the mild degree of TSH elevation (median TSH 3.1-3.8 mU/L) in its subjects. Furthermore, the predictive value of early IQ testing at age 3 years remains uncertain. With these major limitations, the negative primary endpoint nonetheless does not support universal screening for thyroid disease in pregnant women.

Two separate randomized trials evaluating the effect of LT4 treatment upon fetal neurodevelopment are currently in progress. The first is a multicenter, randomized placebocontrolled clinical trial conducted by the National Institutes of Health, which seeks to evaluate the effects of LT4 treatment in women with either subclinical hypothyroidism or isolated hypothyroxinemia on offspring cognition. Preliminary results presented as an oral abstract at the 2016 Annual Meeting of the Society for Maternal Fetal Medicine meeting showed that initiation of LT4 treatment in these women at a mean gestational age of 17 weeks had no effect on child IQ (21). Full results are anticipated in 2017. A separate, large prospective study is also underway in China. The Subclinical Hypothyroid during Early Pregnancy (SHEP) trial will screen 21,500 women planning pregnancy and seek to assess the effects of LT4 treatment initiated before conception in women with iodine deficiency, those with subclinical hypothyroidism, and those positive for TPOAb (588). The results of the study are expected between 2018 and 2020.

Importantly, if a screening paradigm is conclusively proved effective, universal screening of women in the first trimester has been shown to be cost effective (589-592). A study by Dosiou et al. (590) established the cost-effectiveness of universal screening of pregnant women with both TSH and TPO antibodies in the first trimester compared with a strategy of high-risk screening. At base-case analysis, this study assumed that treatment of identified women with TPOAb positivity and subclinical hypothyroidism reduced pregnancy loss and preterm delivery rates as per the randomized clinical trial of Negro and colleagues (28), and that screening resulted in more prompt diagnosis of PPT and hypothyroidism later in life, in the women who developed these disorders. The study did not assume any adverse effects of untreated thyroid disease on child IQ. In sensitivity analyses, even when the benefits of screening were limited to detection and treatment of overt hypothyroidism, screening was shown to be highly costeffective at $<\$ 8000 /$ quality-adjusted life year (QALY). Assumption of benefit for fetal IQ made screening cost-saving. Another recent study from Spain demonstrated similar findings, with an incremental cost-effectiveness ratio of $€ 374.28$ / QALY (592). The cost effectiveness of screening for thyroid disease in pregnancy compares well with that for other widely used screening practices in pregnant women such as cystic fibrosis screening (\$8290/QALY) (593) and prenatal diabetes screening (\$16,689-19,339/QALY) (594).

Finally, it is important to place the screening discussion in the context of worldwide opinion, and acknowledge the recommendations of other Societies. The 2011 ATA pregnancy guidelines and the 2012 Endocrine Society pregnancy guidelines significantly expanded the definition of "high risk" women from that used in earlier editions to include women $>30$ years of age. This expanded definition of high risk encompassed many more women in populations with a high mean maternal pregnancy age. In a 2012 study by Potlukova et al. (595), with a mean maternal age at pregnancy of 31 years, the addition of age 30 or older as a risk factor increased the proportion of women correctly identified in a case-finding strategy from $55.3 \%$ to $85.6 \%$. However, in a recent Chinese population with mean maternal age at pregnancy of 26.6 years, testing using only high-risk criteria missed $82.4 \%$ of women with subclinical hypothyroidism, $28.6 \%$ of women with overt hyperthyroidism, and $74.6 \%$ of women with antithyroid antibodies (596). Member surveys of professional societies have shown that $42.7 \%$ of responders in Latin America and $43 \%$ in Europe perform universal screening $(597,598)$, whereas only $21 \%$ of the Asia-Oceania Thyroid Association members do so (599), and 74\% of ATA members support such an approach (600). In the recent 2014 ETA guidelines, the majority of authors recommended universal screening because of the beneficial effects of treatment for overt hypothyroidism, and the fact that the targeted approach will miss a large percentage of women with subclinical hypothyroidism (601). The Spanish Society of Endocrinology and Nutrition (602) and the Indian Thyroid Society (603) have expressed support for universal screening in early pregnancy or preconception. The Indian National Guidelines recommend testing only high-risk women (604). Finally, the American Society for Reproductive Medicine recommends TSH testing in all infertile women attempting pregnancy and in high-risk women in early pregnancy (605).

Together, these data provide a perplexing dilemma. Studies strongly suggest an increase in pregnancy loss risk associated with elevated maternal TSH concentrations, especially when elevated TPOAb are detected. Similarly, thyroid dysfunction is a prevalent condition that can be diagnosed with readily available and inexpensive tests. However, the effectiveness of LT4 therapy has not yet been conclusively demonstrated. Importantly, many have argued that screening for thyroid dysfunction must occur very early in pregnancy (e.g., 4-7 weeks of gestation) to maximize potential benefits of LT4 treatment upon pregnancy loss rates and possibly neurocognitive development. The largest prospective screening studies thus far have provided data most translatable to typical pregnancy care currently provided 
worldwide, with initial evaluation between 10 and 15 weeks of gestation. This is important to consider because the feasibility of any screening earlier in gestation is unclear.

Further uncertainty surrounds the choice of biochemical test to use for any screening mandate. Most studies identify two dichotomous hypothyroid populations detected in early pregnancy-those with elevated maternal TSH concentrations, but separately from those with decreased FT4 concentrations. Such populations rarely overlap. These findings suggest that the most effective screening strategies may require multimodal testing beyond just TSH evaluation. And, as mentioned above, detection of elevated TPOAb concentrations may ultimately prove the strongest risk factor for adverse outcomes, though no RCT to date has used this as the sole initial testing approach. Furthermore, the only study to date to target treatment of pregnant women with low FT4 concentrations demonstrated no benefit (306).

Therefore, while acknowledging an impressive amount of retrospective data associating thyroid dysfunction with pregnancy harm, the above uncertainties preclude the task force from recommending for or against a universal screening mandate. The outcome of future studies will prove critical for expanding our knowledge, and the task force acknowledged the wealth of data which will become available in the years ahead. In coming to this conclusion, the task force noted that the majority of patients identified through any universal screening process have TSH concentrations between 2.5 and $5.0 \mathrm{mU} / \mathrm{L}-\mathrm{a}$ population in whom a treatment benefit is not well established. Furthermore, such a strategy could have detrimental effects, labeling many patients with a biochemical abnormality, and in many cases leading to initiation of possibly inappropriate long-term treatment.

One task force member (CD) dissented from this recommendation, feeling that universal testing for maternal TSH and anti-TPO antibodies soon after pregnancy confirmation is warranted given the existing support for the obstetrical benefits of treatment, with minimal risk of harm with appropriate monitoring. There might be an additional benefit of treatment on fetal neurodevelopment, as shown in animal studies. Unfortunately, the timing of initiation of LT4 treatment after completion of the first trimester in the RCTs that examine child IQ as the outcome is a very serious design flaw that biases these studies to producing negative results, since they miss the relevant window of opportunity to influence neurodevelopment. Nonetheless, universal screening would allow identification and treatment of all women with overt hypothyroidism, which is prevalent in $0.3 \%-0.5 \%$ of pregnant women $(259,260,583)$ and asymptomatic in about $70 \%$ of patients (606). It would also allow identification and selective treatment of women with autoimmune subclinical hypothyroidism, and appropriate close monitoring of euthyroid women with isolated thyroid autoimmunity, some of whom will develop hypothyroidism during gestation or PPT.

The task force uniformly recommends that health care providers identify all newly pregnant women at high risk for thyroid disease. This would include women with a history of thyroid dysfunction, symptoms or signs of thyroid dysfunction, presence of a goiter, and known thyroid antibody positivity. Other risk factors for thyroid disease include age $>30$ years $(145,607)$, history of diabetes mellitus type 1 (608) or other autoimmune disorders (609), history of pregnancy loss, preterm delivery or infertility $(206,208)$, history of head or neck radiation $(610)$ or prior thyroid surgery $(611,612)$, family history of autoimmune thyroid disease or thyroid dysfunction $(613,614)$, morbid obesity $(615,616)$, use of amiodarone $(617)$, lithium (392), or recent administration of iodinated radiologic contrast (618), two or more prior pregnancies (619), and residing in area of moderate to severe iodine deficiency (620). In these women, measurement of serum TSH concentration should be performed as soon as pregnancy is confirmed, with reflex anti-TPOAb if TSH is $2.5-10 \mathrm{mU} / \mathrm{L}$ (Fig. 1).

While screening women for thyroid disease preconception may also prove beneficial, there are currently no data to support such an approach, and the process of testing such a high volume of women, the majority of whom will not become pregnant, seems impractical. Universal screening for TPOAb in early pregnancy or possibly preconception may also prove an attractive alternative, but it warrants further investigation. The high prevalence of anti-TPO positivity (up to $17 \%$ in reproductive age women), the extensive findings demonstrating increased risks in the anti-TPOAb-positive population, and the fact that this test would also identify women at risk for developing hypothyroidism during gestation (20\%) (147) and PPT (30-50\%) (621), make this test an attractive theoretical consideration. However, no data support this testing algorithm at present.

\section{Question 111: Should women be universally tested for thyroid function before or during pregnancy?}

\section{- RECOMMENDATION 93}

There is insufficient evidence to recommend for or against universal screening for abnormal TSH concentrations in early pregnancy.

\section{No recommendation, insufficient evidence.}

\section{- RECOMMENDATION 94}

There is insufficient evidence to recommend for or against universal screening for abnormal TSH concentrations preconception, with the exception of women planning assisted reproduction or those known to have TPOAb positivity.

\section{No recommendation, insufficient evidence.}

\section{- RECOMMENDATION 95}

Universal screening to detect low FT4 concentrations in pregnant women is not recommended.

Weak recommendation, moderate-quality evidence.

\section{- RECOMMENDATION 96}

All pregnant women should be verbally screened at the initial prenatal visit for any history of thyroid dysfunction, and prior or current use of either thyroid hormone (LT4) or antithyroid medications (MMI, CM, or PTU).

\section{Strong recommendation, high-quality evidence.}

\section{- RECOMMENDATION 97}

All patients seeking pregnancy, or newly pregnant, should undergo clinical evaluation. If any of the following risk factors are identified, testing for serum TSH is recommended: 


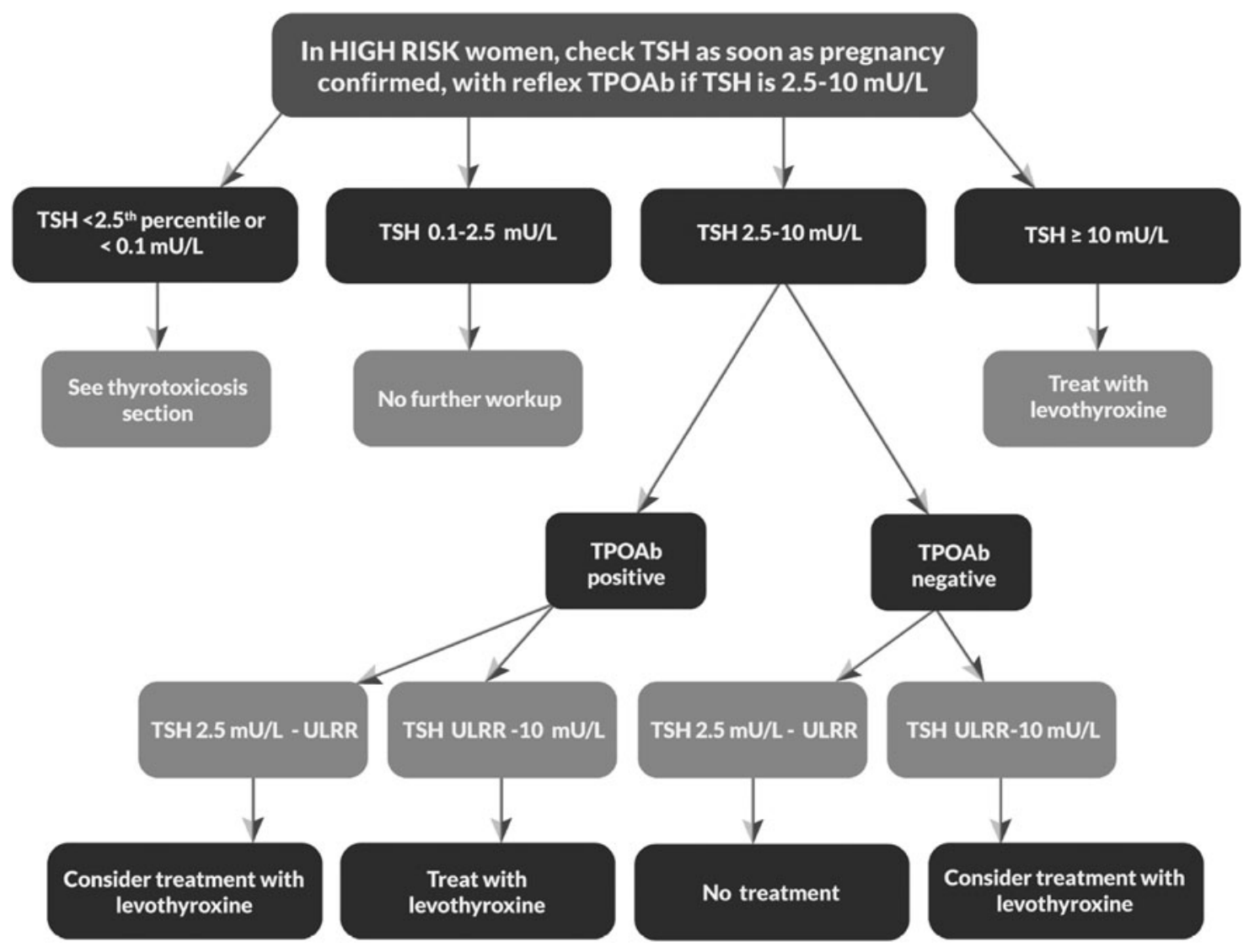

FIG. 1. Testing for thyroid dysfunction in pregnancy. ULRR, upper limit of the reference range.

1. A history of hypothyroidism/hyperthyroidism or current symptoms/signs of thyroid dysfunction

2. Known thyroid antibody positivity or presence of a goiter

3. History of head or neck radiation or prior thyroid surgery

4. Age $>30$ years

5. Type 1 diabetes or other autoimmune disorders

6 . History of pregnancy loss, preterm delivery, or infertility

7. Multiple prior pregnancies $(\geq 2)$

8. Family history of autoimmune thyroid disease or thyroid dysfunction

9. Morbid obesity (BMI $\geq 40 \mathrm{~kg} / \mathrm{m}^{2}$ )

10. Use of amiodarone or lithium, or recent administration of iodinated radiologic contrast

11. Residing in an area of known moderate to severe iodine insufficiency

\section{Strong recommendation, moderate-quality evidence.}

\section{FUTURE RESEARCH DIRECTIONS}

In developing the Guidelines, the task force frequently struggled with the paucity of high-quality double-blinded placebo-controlled trials in the field of thyroid and pregnancy. In fact, only a minority of the 97 recommendations (24\%) in the present Guidelines were graded at the highest American College of Physicians Grading System level of evidence. The Guidelines task force identified topics for future research that will be critical in resolving many of the unanswered questions in the field of thyroid and pregnancy. Of concern to the task force is that most of the double-blind placebo-controlled studies either recently completed, or presently underway, began screening and intervention after the first trimester. As such, these studies will not be able to address the impact of LT4 treatment in the first trimester in women with subclinical hypothyroidism, isolated hypothyroxinemia, or thyroid Ab positivity on the mother and developing fetus. A trial that screens women preconception and then randomizes women with subclinical hypothyroidism, isolated hypothyroxinemia, and isolated thyroid autoimmunity (with normal TSH) to either a treatment or no treatment arm is needed. The task force is aware of the difficulties inherent in performing such a trial, and the ethical challenges to be faced. Nevertheless, we believe that such a trial is feasible, can be ethically performed with appropriate study design and safeguards, and will yield invaluable information related to the optimal care of the pregnant women and the developing fetus. Other areas for future research include:

- A study evaluating the impact of iodine supplementation in pregnant women with the mildest form of iodine deficiency (median UICs $100-150 \mu \mathrm{g} / \mathrm{L}$ ).

- A RCT of early LT4 intervention (at 4-8 weeks) in women with either subclinical hypothyroidism or isolated hypothyroxinemia to determine effects on child IQ.

- A study focused on the effects of iodine supplementation during lactation on infant thyroid function and cognition.

- A study to determine safe upper limits for iodine ingestion in pregnancy and lactation. 
- A comprehensive study to assess the iodine status of pregnant and lactating women in the United States.

- A trial assessing the optimal targeted FT4 level in pregnant women treated for hyperthyroidism.

- Another well powered, prospective, randomized interventional trial of LT4 in euthyroid patients who are anti-TPOAb positive for the prevention of miscarriage and preterm delivery.

- A study to evaluate the impact of LT4 therapy in euthyroid thyroid $\mathrm{Ab}$-positive women with recurrent pregnancy loss.

- Basic and clinical studies aimed at elucidating the mechanisms underlying thyroid Ab-associated adverse pregnancy outcomes.

- Studies examining the effects of $\mathrm{TgAb}$ on pregnancy outcomes.

- A study investigating the best criteria that can be used to predict which patients with hyperthyroidism can safely tapered off antithyroid medication in the first trimester.

- A study evaluating the safest timing of administration of the different ATDs for management of hyperthyroidism in pregnancy.

- Novel ways to differentiate fetal hyperthyroidism from fetal hypothyroidism when a fetal goiter is detected.

\section{ACKNOWLEDGMENTS}

The task force wishes to thank Ms. Bobbi Smith, Executive Director, ATA, and the rest of the ATA staff for their constant help and support, as well as Ms. Sheri Slaughter for her substantial assistance in compiling meeting notes, updating drafts, and final manuscript preparation. We thank Dr. Tim I. Korevaar (Erasmus University, the Netherlands) for his help in compiling Tables 4-7. We would like to thank the ATA members who responded to our survey in preparation for this iteration of the guidelines as well as manuscript review prior to journal submission. These guidelines were funded by the ATA without support from any commercial sources. The following groups reviewed and endorsed the final document: American Association of Clinical Endocrinologists (AACE); American Radium Society (ARS); Brazilian Society of Head and Neck Surgery (BSHNS); Graves' Disease and Thyroid Foundation; International Association of Endocrine Surgeons (IAES); Japan Thyroid Association; The Endocrine Society of Australia (ESA); ThyCa: Thyroid Cancer Survivors' Association, Inc.; and the Thyroid Federation International. The Pediatric Endocrine Society affirms the value of these guidelines.

\section{AUTHOR DISCLOSURE STATEMENT}

These guidelines were funded by the ATA without support from any commercial sources.

CD, GB, HC, JL, PL, SM, SS, and WG have no significant financial or competing interests to disclose.

EP has been a consultant for the Scientific Consulting Company $\mathrm{GmbH}$.

EA has been a consultant for Veracyte.

$\mathrm{RB}$ has received grant and/or research support from Versartis, Lilly, Novo Nordisk and Genentech.
RP has received a speaker honoria from Genzyme and Ipsen as well as serving on the advisory boards of Genzyme, Veracyte and Eisai. Additionally, as patent holder for a test to predict thyroid dysfunction during pregnancy, he recused himself from review and approval of the Screening for Thyroid Dysfunction Before or During Pregnancy (Section XIII).

\section{REFERENCES}

1. Stagnaro-Green A, Abalovich M, Alexander E, Azizi F, Mestman J, Negro R, Nixon A, Pearce EN, Soldin OP, Sullivan S, Wiersinga W, American Thyroid Association Taskforce on Thyroid Disease During Pregnancy and Postpartum 2011 Guidelines of the American Thyroid Association for the diagnosis and management of thyroid disease during pregnancy and postpartum. Thyroid 21:1081-1125.

2. Qaseem A, Snow V, Owens DK, Shekelle P 2010 The development of clinical practice guidelines and guidance statements of the American College of Physicians: summary of methods. Ann Intern Med 153:194-199.

3. van Raaij JM, Vermaat-Miedema SH, Schonk CM, Peek ME, Hautvast JG 1987 Energy requirements of pregnancy in The Netherlands. Lancet 2:953-955.

4. Glinoer D 1997 The regulation of thyroid function in pregnancy: pathways of endocrine adaptation from physiology to pathology. Endocr Rev 18:404-433.

5. Weeke J, Dybkjaer L, Granlie K, Eskjaer Jensen S, Kjaerulff E, Laurberg P, Magnusson B 1982 A longitudinal study of serum TSH, and total and free iodothyronines during normal pregnancy. Acta Endocrinologica 101:531.

6. Baloch Z, Carayon P, Conte-Devolx B, Demers LM, Feldt-Rasmussen U, Henry JF, LiVosli VA, Niccoli-Sire P, John R, Ruf J, Smyth PP, Spencer CA, Stockigt JR, Guidelines Committee, National Academy of Clinical Biochemistry 2003 Laboratory medicine practice guidelines. Laboratory support for the diagnosis and monitoring of thyroid disease. Thyroid 13:3-126.

7. Soldin OP, Tractenberg RE, Hollowell JG, Jonklaas J, Janicic N, Soldin SJ 2004 Trimester-specific changes in maternal thyroid hormone, thyrotropin, and thyroglobulin concentrations during gestation: trends and associations across trimesters in iodine sufficiency. Thyroid 14:1084-1090.

8. Kahric-Janicic N, Soldin SJ, Soldin OP, West T, Gu J, Jonklaas J 2007 Tandem mass spectrometry improves the accuracy of free thyroxine measurements during pregnancy. Thyroid 17:303-311.

9. Orito Y, Oku H, Kubota S, Amino N, Shimogaki K, Hata M, Manki K, Tanaka Y, Sugino S, Ueta M, Kawakita K, Nunotani T, Tatsumi N, Ichihara K, Miyauchi A, Miyake M 2009 Thyroid function in early pregnancy in Japanese healthy women: relation to urinary iodine excretion, emesis, and fetal and child development. J Clin Endocrinol Metab 94:1683-1688.

10. Roti E, Gardini E, Minelli R, Bianconi L, Flisi M 1991 Thyroid function evaluation by different commercially available free thyroid hormone measurement kits in term pregnant women and their newborns. J Endocrinol Invest 14:1-9.

11. Sapin R, D’Herbomez M, Schlienger JL 2004 Free thyroxine measured with equilibrium dialysis and nine immunoassays decreases in late pregnancy. Clin Lab 50:581-584. 
12. Haddow JE, Knight GJ, Palomaki GE, McClain MR, Pulkkinen AJ 2004 The reference range and within-person variability of thyroid stimulating hormone during the first and second trimesters of pregnancy. J Med Screen 11:170-174.

13. Panesar NS, Li CY, Rogers MS 2001 Reference intervals for thyroid hormones in pregnant Chinese women. Ann Clin Biochem 38:329-332.

14. Lockwood CM, Grenache DG, Gronowski AM 2009 Serum human chorionic gonadotropin concentrations greater than 400,000 IU/L are invariably associated with suppressed serum thyrotropin concentrations. Thyroid 19:863-868.

15. De Groot L, Abalovich M, Alexander EK, Amino N, Barbour L, Cobin RH, Eastman CJ, Lazarus JH, Luton D, Mandel SJ, Mestman J, Rovet J, Sullivan S 2012 Management of thyroid dysfunction during pregnancy and postpartum: an Endocrine Society clinical practice guideline. J Clin Endocrinol Metab 97:2543-2565.

16. Yan YQ, Dong ZL, Dong L, Wang FR, Yang XM, Jin XY, Lin LX, Sun YN, Chen ZP 2011 Trimester- and methodspecific reference intervals for thyroid tests in pregnant Chinese women: methodology, euthyroid definition and iodine status can influence the setting of reference intervals. Clin Endocrinol (Oxf) 74:262-269.

17. Li C, Shan Z, Mao J, Wang W, Xie X, Zhou W, Li C, Xu B, Bi L, Meng T, Du J, Zhang S, Gao Z, Zhang X, Yang L, Fan C, Teng W 2014 Assessment of thyroid function during first-trimester pregnancy: what is the rational upper limit of serum TSH during the first trimester in Chinese pregnant women? J Clin Endocrinol Metab 99:73-79.

18. Marwaha RK, Chopra S, Gopalakrishnan S, Sharma B, Kanwar RS, Sastry A, Singh S 2008 Establishment of reference range for thyroid hormones in normal pregnant Indian women. BJOG 115:602-606.

19. Korevaar TI, Schalekamp-Timmermans S, de Rijke YB, Visser WE, Visser W, de Muinck Keizer-Schrama SM, Hofman A, Ross HA, Hooijkaas H, Tiemeier H, BongersSchokking JJ, Jaddoe VW, Visser TJ, Steegers EA, Medici M, Peeters RP 2013 Hypothyroxinemia and TPO-antibody positivity are risk factors for premature delivery: the generation R study. J Clin Endocrinol Metab 98:4382-4390.

20. Moon HW, Chung HJ, Park CM, Hur M, Yun YM 2015 Establishment of trimester-specific reference intervals for thyroid hormones in Korean pregnant women. Ann Lab Med 35:198-204.

21. Casey B. Effect of treatment of maternal subclinical hypothyroidism or hypothyroxinemia on IQ in offspring. [Abstract]. Available at: www.ajog.org/article/S0002-9378(15)01319-8/ pdf (accessed February 9, 2017).

22. Casey BM, Dashe JS, Wells CE, McIntire DD, Leveno KJ, Cunningham FG 2006 Subclinical hyperthyroidism and pregnancy outcomes. Obstet Gynecol 107:337-341.

23. Thienpont LM, Van Uytfanghe K, Beastall G, Faix JD, Ieiri T, Miller WG, Nelson JC, Ronin C, Ross HA, Thijssen JH, Toussaint B, IFCC Working Group on Standardization of Thyroid Function Tests 2010 Report of the IFCC Working Group for Standardization of Thyroid Function Tests; Part 1: Thyroid-stimulating hormone. Clin Chem 56:902-911.

24. Bestwick JP, John R, Maina A, Guaraldo V, Joomun M, Wald NJ, Lazarus JH 2014 Thyroid stimulating hormone and free thyroxine in pregnancy: expressing concentrations as multiples of the median (MoMs). Clin Chim Acta 430:33-37.
25. Toft AD, Beckett GJ 2005 Measuring serum thyrotropin and thyroid hormone and assessing thyroid hormone transport. In: Braverman, LE, Utiger, RD (eds) Werner \& Ingbar's The Thyroid: A Fundamental and Clinical Text. Ninth edition. Lippincott, Williams \& Wilkins, Philadelphia, PA, pp 329-344.

26. Anckaert E, Poppe K, Van Uytfanghe K, Schiettecatte J, Foulon W, Thienpont LM 2010 FT4 immunoassays may display a pattern during pregnancy similar to the equilibrium dialysis ID-LC/tandem MS candidate reference measurement procedure in spite of susceptibility towards binding protein alterations. Clin Chim Acta 411:1348-1353.

27. Sapin R, d'Herbomez M 2003 Free thyroxine measured by equilibrium dialysis and nine immunoassays in sera with various serum thyroxine-binding capacities. Clin Chem 49:1531-1535.

28. Negro R, Formoso G, Mangieri T, Pezzarossa A, Dazzi D, Hassan H 2006 Levothyroxine treatment in euthyroid pregnant women with autoimmune thyroid disease: effects on obstetrical complications. J Clin Endocrinol Metab 91:2587-2591.

29. Lee RH, Spencer CA, Mestman JH, Miller EA, Petrovic I, Braverman LE, Goodwin TM 2009 Free T4 immunoassays are flawed during pregnancy. Am J Obstet Gynecol 200:260.e1-260.e6.

30. Stockigt J 2003 Assessment of thyroid function: towards an integrated laboratory-clinical approach. Clin Biochem Rev 24:109-122.

31. Yue B, Rockwood AL, Sandrock T, La'ulu SL, Kushnir MM, Meikle AW 2008 Free thyroid hormones in serum by direct equilibrium dialysis and online solid-phase extraction-liquid chromatography/tandem mass spectrometry. Clin Chem 54:642-651.

32. Thienpont LM, Van Uytfanghe K, Beastall G, Faix JD, Ieiri T, Miller WG, Nelson JC, Ronin C, Ross HA, Thijssen JH, Toussaint B, IFCC Working Group on Standardization of Thyroid Function Tests 2010 Report of the IFCC Working Group for Standardization of Thyroid Function Tests; part 2: free thyroxine and free triiodothyronine. Clin Chem 56:912-920.

33. Glinoer D 2007 The importance of iodine nutrition during pregnancy. Public Health Nutr 10:1542-1546.

34. Liberman CS, Pino SC, Fang SL, Braverman LE, Emerson CH 1998 Circulating iodide concentrations during and after pregnancy. J Clin Endocrinol Metab 83:3545-3549.

35. Brander L, Als C, Buess H, Haldimann F, Harder M, Hanggi W, Herrmann U, Lauber K, Niederer U, Zurcher T, Burgi U, Gerber H 2003 Urinary iodine concentration during pregnancy in an area of unstable dietary iodine intake in Switzerland. J Endocrinol Invest 26:389-396.

36. Azizi F, Smyth P 2009 Breastfeeding and maternal and infant iodine nutrition. Clin Endocrinol (Oxf) 70:803-809.

37. Andersen S, Karmisholt J, Pedersen KM, Laurberg P 2008 Reliability of studies of iodine intake and recommendations for number of samples in groups and in individuals. Br J Nutr 99:813-818.

38. König F, Andersson M, Hotz K, Aeberli I, Zimmermann MB 2011 Ten repeat collections for urinary iodine from spot samples or 24-hour samples are needed to reliably estimate individual iodine status in women. J Nutr 141:2049-2054.

39. Berghout A, Wiersinga W 1998 Thyroid size and thyroid function during pregnancy: an analysis. Eur J Endocrinol 138:536-542. 
40. Sahin SB, Ogullar S, Ural UM, Ilkkilic K, Metin Y, Ayaz T 2014 Alterations of thyroid volume and nodular size during and after pregnancy in a severe iodine-deficient area. Clin Endocrinol (Oxf) 81:762-768.

41. Delange FM, Dunn JT 2005 Iodine deficiency. In: Braverman LE, Utiger RD (eds) Werner and Ingbar's The Thyroid: A Fundamental and Clinical Text. Ninth edition. Lippincott, Williams and Wilkins, Philadelphia, pp 264 288.

42. World Health Organization/International Council for the Control of the Iodine Deficiency Disorders/United Nations Children's Fund (WHO/ICCIDD/UNICEF) 2007 Assessment of the iodine deficiency disorders and monitoring their elimination. World Health Organization, Geneva.

43. Vermiglio F, Lo Presti VP, Castagna MG, Violi MA, Moleti M, Finocchiaro MD, Mattina F, Artemisia A, Trimarchi F 1999 Increased risk of maternal thyroid failure with pregnancy progression in an iodine deficient area with major iodine deficiency disorders. Thyroid 9:19-24.

44. de Escobar GM, Obregon MJ, del Rey FE 2007 Iodine deficiency and brain development in the first half of pregnancy. Public Health Nutr 10:1554-1570.

45. Costeira MJ, Oliveira P, Santos NC, Ares S, Saenz-Rico B, Morreale de Escobar G, Palha JA 2011 Psychomotor development of children from an iodine-deficient region. $\mathrm{J}$ Pediatr 159:447-453.

46. Zimmermann MB 2012 The effects of iodine deficiency in pregnancy and infancy. Paediatr Perinat Epidemiol 26(Suppl 1): 108-117.

47. Moreno-Reyes R, Glinoer D, Van Oyen H, Vandevijvere S 2013 High prevalence of thyroid disorders in pregnant women in a mildly iodine-deficient country: a populationbased study. J Clin Endocrinol Metab 98:3694-3701.

48. Olivares JL, Olivi GI, Verdasco C, Ortiz VA, Mayer MA, Cresto JC 2012 Low iodine intake during pregnancy: relationship to placental development and head circumference in newborn. Endocrinol Nutr 59:326-330.

49. Lean MI, Lean ME, Yajnik CS, Bhat DS, Joshi SM, Raut DA, Lubree HG, Combet E 2014 Iodine status during pregnancy in India and related neonatal and infant outcomes. Public Health Nutr 17:1353-1362.

50. Vermiglio F, Lo Presti VP, Moleti M, Sidoti M, Tortorella G, Scaffidi G, Castagna MG, Mattina F, Violi MA, Crisa A, Artemisia A, Trimarchi F 2004 Attention deficit and hyperactivity disorders in the offspring of mothers exposed to mild-moderate iodine deficiency: a possible novel iodine deficiency disorder in developed countries. $\mathbf{J}$ Clin Endocrinol Metab 89:6054-6060.

51. Bath SC, Steer CD, Golding J, Emmett P, Rayman MP 2013 Effect of inadequate iodine status in UK pregnant women on cognitive outcomes in their children: results from the Avon Longitudinal Study of Parents and Children (ALSPAC). Lancet 382:331-337.

52. Hynes KL, Otahal P, Hay I, Burgess JR 2013 Mild iodine deficiency during pregnancy is associated with reduced educationaloutcomes in the offspring: 9-year follow-up of the gestational iodine cohort. J Clin Endocrinol Metab 98: $1954-1962$.

53. van Mil NH, Tiemeier H, Bongers-Schokking JJ, Ghassabian A, Hofman A, Hooijkaas H, Jaddoe VW, de Muinck Keizer-Schrama SM, Steegers EA, Visser TJ, Visser W, Ross HA, Verhulst FC, de Rijke YB, SteegersTheunissen RP 2012 Low urinary iodine excretion during early pregnancy is associated with alterations in executive functioning in children. J Nutr 142:2167-2174.

54. Santiago P, Velasco I, Muela JA, Sánchez B, Martínez J, Rodriguez A, Berrio M, Gutierrez-Repiso C, Carreira M, Moreno A, García-Fuentes E, Soriguer F 2013 Infant neurocognitive development is independent of the use of iodised salt oriodine supplements given during pregnancy. Br J Nutr 110:831-839.

55. Trumpff C, De Schepper J, Tafforeau J, Van Oyen H, Vanderfaeillie J, Vandevijvere S 2013 Mild iodine deficiency in pregnancy in Europe and its consequences for cognitive and psychomotor development of children: a review. J Trace Elem Med Biol 27:174-183.

56. Soldin OP, Soldin SJ, Pezzullo JC 2003 Urinary iodine percentile ranges in the United States. Clin Chim Acta 328: $185-190$.

57. Caldwell KL, Jones R, Hollowell JG 2005 Urinary iodine concentration: United States National Health And Nutrition Examination Survey 2001-2002. Thyroid 15:692699.

58. Caldwell KL, Makhmudov AA, Ely EK, Jarrett JM, Henahan D, Jones RL 2009 Iodine status of the U.S. population, NHANES 2005-2006. Thyroid 19:S26-S27.

59. Caldwell KL, Miller GA, Wang RY, Jain RB, Jones RL 2008 Iodine status of the U.S. population, National Health and Nutrition Examination Survey 2003-2004. Thyroid 18:1207-1214.

60. Hollowell JG, Staehling NW, Hannon WH, Flanders DW, Gunter EW, Maberly GF, Braverman LE, Pino S, Miller DT, Garbe PL, DeLozier DM, Jackson RJ 1998 Iodine nutrition in the United States. Trends and public health implications: iodine excretion data from National Health and Nutrition Examination Surveys I and III (1971-1974 and 1988-1994). J Clin Endocrinol Metab 83:3401-3408.

61. Caldwell KL, Makhmudov A, Ely E, Jones RL, Wang RY 2011 Iodine status of the U.S. population, National Health and Nutrition Examination Survey, 2005-2006 and 20072008. Thyroid 21:419-427.

62. Caldwell KL, Pan Y, Mortensen ME, Makhmudov A, Merrill L, Moye J 2013 Iodine status in pregnant women in the National Children's Study and in U.S. women (1544 years), National Health and Nutrition Examination Survey 2005-2010. Thyroid 23:927-937.

63. Pearce EN, Leung AM, Blount BC, Bazrafshan HR, He X, Pino S, Valentin-Blasini L, Braverman LE 2007 Breast milk iodine and perchlorate concentrations in lactating Boston-area women. J Clin Endocrinol Metab 92:16731677.

64. Kirk AB, Martinelango PK, Tian K, Dutta A, Smith EE, Dasgupta PK 2005 Perchlorate and iodide in dairy and breast milk. Environ Sci Technol 39:2011-2017.

65. Gordon JH, Leung AM, Hale AR, Pearce EN, Braverman LE, He X, Belfort MB, Nelson SM, Brown RS 2014 No difference in urinary iodine concentrations between Boston-area breastfed and formula-fed infants. Thyroid 24:1309-1313.

66. Pearce EN, Andersson M, Zimmermann MB 2013 Global iodine nutrition: where do we stand in 2013? Thyroid 23:523-528.

67. Jaiswal N, Melse-Boonstra A, Sharma SK, Srinivasan K, Zimmermann MB 2015 The iodized salt programme in Bangalore, India provides adequate iodine intakes in pregnant women and more-than-adequate iodine intakes in their children. Public Health Nutr 18:403-413. 
68. Majumder A, Jaiswal A, Chatterjee 2014 Prevalence of iodine deficiency among pregnant and lactating women: experience in Kolkata. Indian $\mathbf{J}$ Endocrinol Metab 18:486-490.

69. Patey-Pirra S, Keriel-Gascou M, Borson-Chazot F 2014 Benefits and risks of iodine supplementation during pregnancy: a review ofobservational and experimental studies in mild-to-moderate iodine deficiencyareas. Rev Epidemiol Sante Publique 62:65-74.

70. Rydbeck F, Bottai M, Tofail F, Persson LÅ, Kippler M 2014 Urinary iodine concentrations of pregnant women in rural Bangladesh: a longitudinal study. J Expo Sci Environ Epidemiol 24:504-509.

71. Elahi S, Nagra SA 2014 Low maternal iodine intake and early pregnancy hypothyroxinemia: possible repercussions for children. Indian J Endocrinol Metab 18:526-530.

72. Wei Z, Wang W, Zhang J, Zhang X, Jin L, Yu X 2015 Urinary iodine level and its determinants in pregnant women of Shanghai, China. Br J Nutr 113:1427-1432.

73. Shi X, Han C, Li C, Mao J, Wang W, Xie X, Li C, Xu B, Meng T, Du J, Zhang S, Gao Z, Zhang X, Fan C, Shan Z, Teng W 2015 Optimal and safe upper limits of iodine intake for early pregnancy in iodine-sufficient regions: a cross-sectional study of 7,190 pregnant women in China. J Clin Endocrinol Metab 100:1630-1638.

74. Cho YY, Kim HJ, Oh SY, Choi SJ, Lee SY, Joung JY, Jeong DJ, Sohn SY, Chung JH, Roh CR, Kim SW 2016 Iodine status in healthy pregnant women in Korea: a first report. Eur J Nutr 55:469-475.

75. Sadou H, Seyfoulaye A, Malam Alma M, Daouda H 2014 Inadequate status of iodine nutrition among pregnant women residing in three districts of Niamey, the Niger Republic's capital. Matern Child Nutr 10:650-656.

76. Kedir H, Berhane Y, Worku A 2014 Subclinical iodine deficiency among pregnant women in Haramaya district, Eastern Ethiopia: a community-based study. J Nutr Metab 2014:878926.

77. Amouzegar A, Khazan M, Hedayati M, Azizi F 2014 An assessment of the iodine status and the correlation between iodine nutritionand thyroid function during pregnancy in an iodine sufficient area. Eur J Clin Nutr 68:397-400.

78. Ferreira SM, Navarro AM, Magalhães PK, Maciel LM 2014 Iodine insufficiency in pregnant women from the State of São Paulo. Arq Bras Endocrinol Metabol 58:282287.

79. Jiskra J, Fait T, Bílek R, Krátký J, Bartáková J, Lukáš J, Límanová Z, Telička Z, Zamrazil V, Potluková E 2014 Mild iodine deficiency in women after spontaneous abortions living in an iodine-sufficient area of Czech Republic: prevalence and impact on reproductive health. Clin Endocrinol (Oxf) 80:452-458.

80. Andersen SL, Sørensen LK, Krejbjerg A, Møller M, Laurberg P 2013 Iodine deficiency in Danish pregnant women. Dan Med J 60:A4657.

81. Raverot V, Bournaud C, Sassolas G, Orgiazzi J, Claustrat F, Gaucherand P, Mellier G, Claustrat B, Borson-Chazot F, Zimmermann M 2012 Pregnant French women living in the Lyon area are iodine deficient and have elevated serum thyroglobulin concentrations. Thyroid 22:522-528.

82. Konrade I, Kalere I, Strele I, Makrecka-Kuka M, Jekabsone A, Tetere E, Veisa V, Gavars D, Rezeberga D, Pīāangs V, Lejnieks A, Dambrova M 2015 Iodine deficiency during pregnancy: a national cross-sectional survey in Latvia. Public Health Nutr 18:2990-2997.
83. Brantsæter AL, Abel MH, Haugen M, Meltzer HM 2013 Risk of suboptimal iodine intake in pregnant Norwegian women. Nutrients 5:424-440.

84. Aguayo A, Grau G, Vela A, Aniel-Quiroga A, Espada M, Martul P, Castaño L, Rica I 2013 Urinary iodine and thyroid function in a population of healthy pregnant women in the North of Spain. J Trace Elem Med Biol 27:302-306.

85. Bath SC, Sleeth ML, McKenna M, Walter A, Taylor A, Rayman MP 2014 Iodine intake and status of UK women of childbearing age recruited at the University of Surrey in the winter. Br J Nutr 112:1715-1723.

86. Pharoah PO, Buttfield IH, Hetzel BS 1971 Neurological damage to the fetus resulting from severe iodine deficiency during pregnancy. Lancet 1:308-310.

87. O'Donnell KJ, Rakeman MA, Zhi-Hong D, Xue-Yi C, Mei ZY, DeLong N, Brenner G, Tai M, Dong W, DeLong GR 2002 Effects of iodine supplementation during pregnancy on child growth and development at school age. Dev Med Child Neurol 44:76-81.

88. Fierro-Benitez R, Cazar R, Stanbury JB, Rodriguez P, Garces F, Fierro-Renoy F, Estrella E 1988 Effects on school children of prophylaxis of mothers with iodized oil in an area of iodine deficiency. J Endocrinol Invest 11:327-335.

89. Cao XY, Jiang XM, Dou ZH, Rakeman MA, Zhang M, O’Donnell K, Ma T, Amette K, DeLong N, DeLong GR 1994 Timing of vulnerability of the brain to iodine deficiency in endemic cretinism. N Engl J Med 331:17391744.

90. DeLong GR, Leslie PW, Wang SH, Jiang XM, Zhang ML, Rakeman M, Jiang JY, Ma T, Cao XY 1997 Effect on infant mortality of iodination of irrigation water in a severely iodine-deficient area of China. Lancet 350:771773.

91. Chaouki ML, Benmiloud M 1994 Prevention of iodine deficiency disorders by oral administration of lipiodol during pregnancy. Eur J Endocrinol 130:547-551.

92. Anees M, Anis RA, Yousaf S, Murtaza I, Sultan A, Arslan M, Shahab M 2015 Effect of maternal iodine supplementation on thyroid function and birth outcome in goiter endemic areas. Curr Med Res Opin Feb 13:1-8.

93. Bouhouch RR, Bouhouch S, Cherkaoui M, Aboussad A, Stinca S, Haldimann M, Andersson M, Zimmermann MB 2014 Direct iodine supplementation of infants versus supplementation of their breastfeeding mothers: a doubleblind, randomised, placebo-controlled trial. Lancet Diabetes Endocrinol 2:197-209.

94. Antonangeli L, Maccherini D, Cavaliere R, Di Giulio C, Reinhardt B, Pinchera A, Aghini-Lombardi F 2002 Comparison of two different doses of iodide in the prevention of gestational goiter in marginal iodine deficiency: a longitudinal study. Eur J Endocrinol 147:29-34.

95. Berbel P, Mestre JL, Santamaria A, Palazon I, Franco A, Graells M, Gonzalez-Torga A, de Escobar GM 2009 Delayed neurobehavioral development in children born to pregnant women with mild hypothyroxinemia during the first month of gestation: the importance of early iodine supplementation. Thyroid 19:511-519.

96. Glinoer D, De Nayer P, Delange F, Lemone M, Toppet V, Spehl M, Grun JP, Kinthaert J, Lejeune B 1995 A randomized trial for the treatment of mild iodine deficiency during pregnancy: maternal, neonatal effects. J Clin Endocrinol Metab 80:258-269. 
97. Liesenkotter KP, Gopel W, Bogner U, Stach B, Gruters A 1996 Earliest prevention of endemic goiter by iodine supplementation during pregnancy. Eur J Endocrinol 134: 443-448.

98. Nohr SB, Laurberg P 2000 Opposite variations in maternal and neonatal thyroid function induced by iodine supplementation during pregnancy. J Clin Endocrinol Metab 85:623-627.

99. Pedersen KM, Laurberg P, Iversen E, Knudsen PR, Gregersen HE, Rasmussen OS, Larsen KR, Eriksen GM, Johannesen PL 1993 Amelioration of some pregnancyassociated variations in thyroid function by iodine supplementation. J Clin Endocrinol Metab 77:1078-1083.

100. Romano R, Jannini EA, Pepe M, Grimaldi A, Olivieri M, Spennati P, Cappa F, D'Armiento M 1991 The effects of iodoprophylaxis on thyroid size during pregnancy. Am J Obstet Gynecol 164:482-485.

101. Velasco I, Carreira M, Santiago P, Muela JA, GarciaFuentes E, Sanchez-Munoz B, Garriga MJ, GonzalezFernandez MC, Rodriguez A, Caballero FF, Machado A, Gonzalez-Romero S, Anarte MT, Soriguer F 2009 Effect of iodine prophylaxis during pregnancy on neurocognitive development of children during the first two years of life. J Clin Endocrinol Metab 94:3234-3241.

102. Sukkhojaiwaratkul D, Mahachoklertwattana P, Poomthavorn P, Panburana P, Chailurkit LO, Khlairit P, Pongratanakul S 2014 Effects of maternal iodine supplementation during pregnancy and lactation oniodine status and neonatal thyroid-stimulating hormone. J Perinatol 34:594-598.

103. Rebagliato M, Murcia M, Alvarez-Pedrerol M, Espada M, Fernández-Somoano A, Lertxundi N, Navarrete-Muñoz EM, Forns J, Aranbarri A, Llop S, Julvez J, Tardón A, Ballester F 2013 Iodine supplementation during pregnancy and infant neuropsychological development. INMA Mother and Child Cohort Study. Am J Epidemiol 177: 944-953.

104. Vandevijvere S, Amsalkhir S, Mourri AB, Van Oyen H, Moreno-Reyes R 2013 Iodine deficiency among Belgian pregnant women not fully corrected by iodine-containing multivitamins: a national cross-sectional survey. Br J Nutr 109:2276-2284.

105. Taylor PN, Okosieme OE, Dayan CM, Lazarus JH 2013 Therapy of endocrine disease: impact of iodine supplementation inmild-to-moderate iodine deficiency: systematic review and meta-analysis. Eur $\mathrm{J}$ Endocrinol 170: R1-R15.

106. Zhou SJ, Anderson AJ, Gibson RA, Makrides M 2013 Effect of iodine supplementation in pregnancy on child development and otherclinical outcomes: a systematic review of randomized controlled trials. Am J Clin Nutr 98:1241-1254.

107. Bath SC, Rayman MP 2015 A review of the iodine status of UK pregnant women and its implications for the offspring. Environ Geochem Health 37:619-629.

108. Stagnaro-Green A, Pearce EN 2013 Iodine and pregnancy: a call to action. Lancet 382:292-293.

109. Bougma K, Aboud FE, Harding KB, Marquis GS 2013 Iodine and mental development of children 5 years old and under: a systematic review and meta-analysis. $\mathrm{Nu}-$ trients 5:1384-1416.

110. Menéndez Torre E, Delgado Alvarez E, Rabal Artal A, Suárez GutiérrezL, Rodríguez Caballero MG, Ares Blanco J, Díaz Naya L, Fernández Fernández JC 2014 Iodine nutrition in pregnant women from Oviedo area. Is iodine supplementation necessary? Endocrinol Nutr 61:404-409.

111. Trumbo P, Yates AA, Schlicker S, Poos M 2001 Dietary reference intakes: vitamin $\mathrm{A}$, vitamin $\mathrm{K}$, arsenic, boron, chromium, copper, iodine, iron, manganese, molybdenum, nickel, silicon, vanadium, and zinc. J Am Diet Assoc 101: 294-301.

112. Maalouf J, Barron J, Gunn JP, Yuan K, Perrine CG, Cogswell ME 2015 Iodized salt sales in the United States. Nutrients 7:1691-1695.

113. Galton DM, Petersson LG, Erb HN 1986 Milk iodine residues in herds practicing iodophor premilking teat disinfection. J Dairy Sci 69:267-271.

114. Conrad LM 3rd, Hemken RW 1978 Milk iodine as influenced by an iodophor teat dip. J Dairy Sci 61:776-780.

115. Pearce EN, Pino S, He X, Bazrafshan HR, Lee SL, Braverman LE 2004 Sources of dietary iodine: bread, cows' milk, and infant formula in the Boston area. J Clin Endocrinol Metab 89:3421-3424.

116. Haldimann M, Alt A, Blanc A, Blondeau K 2005 Iodine content of food groups. J Food Comp Anal 18:461-471.

117. Public Health Committee of the American Thyroid Association, Becker DV, Braverman LE, Delange F, Dunn JT, Franklyn JA, Hollowell JG, Lamm SH, Mitchell ML, Pearce E, Robbins J, Rovet JF 2006 Iodine supplementation for pregnancy and lactation-United States and Canada: recommendations of the American Thyroid Association. Thyroid 16:949-951.

118. Obican SG, Jahnke GD, Soldin OP, Scialli AR 2012 Teratology public affairs committee position paper: iodine deficiency in pregnancy. Birth Defects Res A Clin Mol Teratol 94:677-682.

119. Council on Environmental Health, Rogan WJ, Paulson JA, Baum C, Brock-Utne AC, Brumberg HL, Campbell CC, Lanphear BP, Lowry JA, Osterhoudt KC, Sandel MT, Spanier A, Trasande L 2014 Iodine deficiency, pollutant chemicals, and the thyroid: new information on an old problem. Pediatrics 133:1163-1166.

120. Leung AM, Lamar A, He X, Braverman LE, Pearce EN 2011 Iodine status and thyroid function of Boston-area vegetarians and vegans. J Clin Endocrinol Metab 96: E1303-E1307.

121. Gregory CO, Serdula MK, Sullivan KM 2009 Use of supplements with and without iodine in women of childbearing age in the United States. Thyroid 19:1019-1020.

122. Leung AM, Pearce EN, Braverman LE 2009 Iodine content of prenatal multivitamins in the United States. N Engl J Med 360:939-940.

123. U.S. Salt Institute 2007 Iodized salt. Available at http:// www.saltinstitute.org/Uses-benefits/Salt-in-Food/Essentialnutrient/Iodized-salt (accessed September 12, 2011).

124. Pennington JA 1990 A review of iodine toxicity reports. J Am Diet Assoc 90:1571-1581.

125. Wolff J, Chaikoff IL 1948 Plasma inorganic iodide as a homeostatic regulator of thyroid function. J Biol Chem 174:555-564.

126. Eng PH, Cardona GR, Fang SL, Previti M, Alex S, Carrasco N, Chin WW, Braverman LE 1999 Escape from the acute Wolff-Chaikoff effect is associated with a decrease in thyroid sodium/iodide symporter messenger ribonucleic acid and protein. Endocrinology 140:3404-3410.

127. Fisher DA, Klein AH 1981 Thyroid development and disorders of thyroid function in the newborn. $\mathrm{N}$ Engl $\mathrm{J}$ Med 304:702-712. 
128. Theodoropoulos T, Braverman LE, Vagenakis AG 1979 Iodide-induced hypothyroidism: a potential hazard during perinatal life. Science 205:502-503.

129. Fang MC, Stafford RS, Ruskin JN, Singer DE 2004 National trends in antiarrhythmic and antithrombotic medication use in atrial fibrillation. Arch Intern Med 164:5560 .

130. Aiba M, Ninomiya J, Furuya $K$, Arai H, Ishikawa $H$, Asaumi S, Takagi A, Ohwada S, Morishita Y 1999 Induction of a critical elevation of povidone-iodine absorption in the treatment of a burn patient: report of a case. Surg Today 29:157-159.

131. Leung AM, Avram AM, Brenner AV, Duntas LH, Ehrenkranz J, Hennessey JV, Lee SL, Pearce EN, Roman SA, Stagnaro-Green A, Sturgis EM, Sundaram K, Thomas MJ, Wexler JA 2015 Potential risks of excess iodine ingestion and exposure: statement by the American Thyroid Association public health committee. Thyroid 25:145-146.

132. Aakre I, Bjøro T, Norheim I, Strand TA, Barikmo I, Henjum S 2015 Excessive iodine intake and thyroid dysfunction among lactating Saharawi women. J Trace Elem Med Biol 31:279-284.

133. Sang Z, Wei W, Zhao N, Zhang G, Chen W, Liu H, Shen J, Liu J, Yan Y, Zhang W 2012 Thyroid dysfunction during late gestation is associated with excessive iodine intake in pregnant women. J Clin Endocrinol Metab 97:E1363-E1369.

134. Chen W, Sang Z, Tan L, Zhang S, Dong F, Chu Z, Wei W, Zhao N, Zhang G, Yao Z, Shen J, Zhang W 2015 Neonatal thyroid function born to mothers living with long-term excessive iodine intake from drinking water. Clin Endocrinol (Oxf) 83:399-404.

135. Medici M, Ghassabian A, Visser W, de Muinck KeizerSchrama SM, Jaddoe VW, Visser WE, Hooijkaas H, Hofman A, Steegers EA, Bongers-Schokking JJ, Ross HA, Tiemeier H, Visser TJ, de Rijke YB, Peeters RP 2014 Women with high early pregnancy urinary iodine levels have an increased risk ofhyperthyroid newborns: the population-based Generation R Study. Clin Endocrinol (Oxf) 80:598-606.

136. Thaker VV, Leung AM, Braverman LE, Brown RS, Levine B 2014 Iodine-induced hypothyroidism in full-term infants with congenital heart disease: more common than currently appreciated? J Clin Endocrinol Metab 99:35213526.

137. Ashoor G, Maiz N, Rotas M, Jawdat F, Nicolaides KH 2010 Maternal thyroid function at 11 to 13 weeks of gestation and subsequent fetal death. Thyroid 20:989-993.

138. Benhadi N, Wiersinga WM, Reitsma JB, Vrijkotte TG, van der Wal MF, Bonsel GJ 2007 Ethnic differences in TSH but not in free T4 concentrations or TPO antibodies during pregnancy. Clin Endocrinol (Oxf) 66:765-770.

139. La'ulu SL, Roberts WL 2007 Second-trimester reference intervals for thyroid tests: the role of ethnicity. Clin Chem 53:1658-1664.

140. Abbassi-Ghanavati M, Casey BM, Spong CY, McIntire DD, Halvorson LM, Cunningham FG 2010 Pregnancy outcomes in women with thyroid peroxidase antibodies. Obstet Gynecol 116(2 Pt 1):381-386.

141. Wasserman EE, Nelson K, Rose NR, Eaton W, Pillion JP, Seaberg E, Talor MV, Burek L, Duggan A, Yolken RH 2008 Maternal thyroid autoantibodies during the third trimester and hearing deficits in children: an epidemiologic assessment. Am J Epidemiol 167:701-710.
142. Pearce EN, Oken E, Gillman MW, Lee SL, Magnani B, Platek D, Braverman LE 2008 Association of firsttrimester thyroid function test values with thyroperoxidase antibody status, smoking, and multivitamin use. Endocr Pract 14:33-39.

143. Moleti M, Lo Presti VP, Mattina F, Mancuso A, De Vivo A, Giorgianni G, Di Bella B, Trimarchi F, Vermiglio F 2009 Gestational thyroid function abnormalities in conditions of mild iodine deficiency: early screening versus continuous monitoring of maternal thyroid status. Eur $\mathrm{J}$ Endocrinol 160:611-617.

144. McElduff A, Morris J 2008 Thyroid function tests and thyroid autoantibodies in an unselected population of women undergoing first trimester screening for aneuploidy. Aust N Z J Obstet Gynaecol 48:478-480.

145. Hollowell JG, Staehling NW, Flanders WD, Hannon WH, Gunter EW, Spencer CA, Braverman LE 2002 Serum TSH, T(4), and thyroid antibodies in the United States population (1988 to 1994): National Health and Nutrition Examination Survey (NHANES III). J Clin Endocrinol Metab 87:489-499.

146. Unuane D, Velkeniers B, Anckaert E, Schiettecatte J, Tournaye H, Haentjens P, Poppe K 2013 Thyroglobulin autoantibodies: is there any added value in the detection of thyroid autoimmunity in women consulting for fertility treatment? Thyroid 23:1022-1028.

147. Glinoer D, Riahi M, Grün JP, Kinthaert J 1994 Risk of subclinical hypothyroidism in pregnant women with asymptomatic autoimmune thyroid disorders. J Clin Endocrinol Metab 79:197-204.

148. Seror J, Amand G, Guibourdenche J, Ceccaldi PF, Luton D 2014 Anti-TPO antibodies diffusion through the placental barrier during pregnancy. PLoS One 9(1):e84647.

149. Gartner R, Gasnier BC, Dietrich JW, Krebs B, Angstwurm MW 2002 Selenium supplementation in patients with autoimmune thyroiditis decreases thyroid peroxidase antibodies concentrations. J Clin Endocrinol Metab 87:1687-1691.

150. Duntas LH, Mantzou E, Koutras DA 2003 Effects of a six month treatment with selenomethionine in patients with autoimmune thyroiditis. Eur J Endocrinol 148:389-393.

151. Mazokopakis EE, Papadakis JA, Papadomanolaki MG, Batistakis AG, Giannakopoulos TG, Protopapadakis EE, Ganotakis ES 2007 Effects of 12 months treatment with L-selenomethionine on serum anti-TPO Levels in Patients with Hashimoto's thyroiditis. Thyroid 17:609-612.

152. Fan Y, Xu S, Zhang H, Cao W, Wang K, Chen G, Di H, Cao M, Liu C 2014 Selenium supplementation for autoimmune thyroiditis: a systematic review and metaanalysis. Int J Endocrinol 2014:904573.

153. Karanikas G, Schuetz M, Kontur S, Duan H, Kommata S, Schoen R, Antoni A, Kletter K, Dudczak R, Willheim M 2008 No immunological benefit of selenium in consecutive patients with autoimmune thyroiditis. Thyroid 18:7-12.

154. Negro R, Greco G, Mangieri T, Pezzarossa A, Dazzi D, Hassan H 2007 The influence of selenium supplementation on postpartum thyroid status in pregnant women with thyroid peroxidase autoantibodies. J Clin Endocrinol Metab 92:1263-1268.

155. Mao J, Pop VJ, Bath SC, Vader HL, Redman CW, Rayman MP 2016 Effect of low-dose selenium on thyroid autoimmunity and thyroid function in UK pregnant women with mild-to-moderate iodine deficiency. Eur J Nutr 55:55-61.

156. Stranges S, Marshall JR, Natarajan R, Donahue RP, Trevisan M, Combs GF, Cappuccio FP, Ceriello A, Reid ME 
2007 Effects of long-term selenium supplementation on the incidence of type 2 diabetes: a randomized trial. Ann Intern Med 147:217-223.

157. Ellish NJ, Saboda K, O'Connor J, Nasca PC, Stanek EJ, Boyle C 1996 A prospective study of early pregnancy loss. Hum Reprod 11:406-412.

158. Wilcox AJ, Weinberg CR, O'Connor JF, Baird DD, Schlatterer JP, Canfield RE, Armstrong EG, Nisula BC 1988 Incidence of early loss of pregnancy. N Engl J Med 319:189-194.

159. Toth B, Jeschke U, Rogenhofer N, Scholz C, Wurfel W, Thaler CJ, Makrigiannakis A 2010 Recurrent miscarriage: current concepts in diagnosis and treatment. J Reprod Immunol 85:25-32.

160. Dudley DJ 2007 Diabetic-associated stillbirth: incidence, pathophysiology, and prevention. Clin Perinatol 34:611626, vii.

161. De Vivo A, Mancuso A, Giacobbe A, Moleti M, Maggio Savasta L, De Dominici R, Priolo AM, Vermiglio F 2010 Thyroid function in women found to have early pregnancy loss. Thyroid 20:633-637.

162. Stagnaro-Green A, Roman SH, Cobin RH, el-Harazy E, Alvarez-Marfany M, Davies TF 1990 Detection of at-risk pregnancy by means of highly sensitive assays for thyroid autoantibodies. JAMA 264:1422-1425.

163. Chen L, Hu R 2011 Thyroid autoimmunity and miscarriage: a meta-analysis. Clin Endocrinol (Oxf) 74:513-519.

164. Thangaratinam S, Tan A, Knox E, Kilby MD, Franklyn J, Coomarasamy A 2011 Association between thyroid autoantibodies and miscarriage and preterm birth: metaanalysis of evidence. BMJ 342:d2616.

165. Lee YL, Ng HP, Lau KS, Liu WM, O WS, Yeung WS, Kung AW 2009 Increased fetal abortion rate in autoimmune thyroid disease is related to circulating TPO autoantibodies in an autoimmune thyroiditis animal model. Fertil Steril 91:2104-2109.

166. Imaizumi M, Pritsker A, Kita M, Ahmad L, Unger P, Davies T 2001 Pregnancy and murine thyroiditis: thyroglobulin immunization leads to fetal loss in specific allogeneic pregnancies. Endocrinology 142:823-829.

167. Matalon ST, Blank M, Levy Y, Carp HJ, Arad A, Burek L, Grunebaum E, Sherer Y, Ornoy A, Refetoff S, Weiss RE, Rose NR, Shoenfeld Y 2003 The pathogenic role of antithyroglobulin antibody on pregnancy: evidence from an active immunization model in mice. Hum Reprod 18: 1094-1099.

168. Twig G, Shina A, Amital H, Shoenfeld Y 2012 Pathogenesis of infertility and recurrent pregnancy loss in thyroid autoimmunity. J Autoimmun 38:J275-J281.

169. Lee RM, Silver RM 2000 Recurrent pregnancy loss: summary and clinical recommendations. Semin Reprod Med 18:433-440.

170. Baek KH, Lee EJ, Kim YS 2007 Recurrent pregnancy loss: the key potential mechanisms. Trends Mol Med 13:310-317.

171. Iravani AT, Saeedi MM, Pakravesh J, Hamidi S, Abbasi M 2008 Thyroid autoimmunity and recurrent spontaneous abortion in Iran: a case-control study. Endocr Pract 14: 458-464.

172. Kutteh WH, Yetman DL, Carr AC, Beck LA, Scott RT Jr 1999 Increased prevalence of antithyroid antibodies identified in women with recurrent pregnancy loss but not in women undergoing assisted reproduction. Fertil Steril 71:843-848.
173. Esplin MS, Branch DW, Silver R, Stagnaro-Green A 1998 Thyroid autoantibodies are not associated with recurrent pregnancy loss. Am J Obstet Gynecol 179(6 Pt 1):15831586.

174. Pratt DE, Kaberlein G, Dudkiewicz A, Karande V, Gleicher N 1993 The association of antithyroid antibodies in euthyroid nonpregnant women with recurrent first trimester abortions in the next pregnancy. Fertil Steril 60: 1001-1005.

175. Rushworth FH, Backos M, Rai R, Chilcott IT, Baxter N, Regan L 2000 Prospective pregnancy outcome in untreated recurrent miscarriers with thyroid autoantibodies. Hum Reprod 15:1637-1639.

176. Lata K, Dutta P, Sridhar S, Rohilla M, Srinivasan A, Prashad GR, Shah VN, Bhansali A 2013 Thyroid autoimmunity and obstetric outcomes in women with recurrent miscarriage: a case-control study. Endocr Connect 2:118-124.

177. van den Boogaard E, Vissenberg R, Land JA, van Wely M, van der Post JA, Goddijn M, Bisschop PH 2011 Significance of (sub)clinical thyroid dysfunction and thyroid autoimmunity before conception and in early pregnancy: a systematic review. Hum Reprod Update 17:605-619.

178. De Carolis C, Greco E, Guarino MD, Perricone C, Dal Lago A, Giacomelli R, Fontana L, Perricone R 2004 Anti-thyroid antibodies and antiphospholipid syndrome: evidence of reduced fecundity and of poor pregnancy outcome in recurrent spontaneous aborters. Am J Reprod Immunol 52:263-266.

179. Kim NY, Cho HJ, Kim HY, Yang KM, Ahn HK, Thornton S, Park JC, Beaman K, Gilman-Sachs A, Kwak-Kim J 2011 Thyroid autoimmunity and its association with cellular and humoral immunity in women with reproductive failures. Am J Reprod Immunol 65:78-87.

180. Lepoutre T, Debiève F, Gruson D, Daumerie C 2012 Reduction of miscarriages through universal screening and treatment of thyroid autoimmune diseases. Gynecol Obstet Invest 74:265-273.

181. Kiprov DD, Nachtigall RD, Weaver RC, Jacobson A, Main EK, Garovoy MR 1996 The use of intravenous immunoglobulin in recurrent pregnancy loss associated with combined alloimmune and autoimmune abnormalities. Am J Reprod Immunol 36:228-234.

182. Stricker RB, Steinleitner A, Bookoff CN, Weckstein LN, Winger EE 2000 Successful treatment of immunologic abortion with low-dose intravenous immunoglobulin. Fertil Steril 73:536-540.

183. Vaquero E, Lazzarin $\mathrm{N}$, De Carolis C, Valensise $\mathrm{H}$, Moretti C, Ramanini C 2000 Mild thyroid abnormalities and recurrent spontaneous abortion: diagnostic and therapeutical approach. Am J Reprod Immunol 43:204-208.

184. Martin JA, Hamilton BE, Osterman MJK, Curtin SC, Mathews T 2015 Births: final data for 2013. Vital Statistics Reports. Available at http://www.cdc.gov/nchs/data/ nvsr/nvsr64/nvsr64_01.pdf (accessed February 28, 2015).

185. Institute of Medicine (US) Committee on Understanding Premature Birth and Assuring Healthy Outcomes 2007 Preterm Birth: Causes, Consequences, and Prevention. Behrman RE, Butler AS (eds). National Academies Press, Washington, DC.

186. Goldenberg RL, Culhane JF, Iams JD, Romero R 2008 Epidemiology and causes of preterm birth. Lancet 371: 75-84.

187. Glinoer D, Soto MF, Bourdoux P, Lejeune B, Delange F, Lemone M, Kinthaert J, Robijn C, Grun JP, de Nayer P 
1991 Pregnancy in patients with mild thyroid abnormalities: maternal and neonatal repercussions. J Clin Endocrinol Metab 73:421-427.

188. Ghafoor F, Mansoor M, Malik T, Malik MS, Khan AU, Edwards R, Akhtar W 2006 Role of thyroid peroxidase antibodies in the outcome of pregnancy. J Coll Physicians Surg Pak 16:468-471.

189. Iijima T, Tada H, Hidaka Y, Mitsuda N, Murata Y, Amino N 1997 Effects of autoantibodies on the course of pregnancy and fetal growth. Obstet Gynecol 90:364-369.

190. Haddow JE, Cleary-Goldman J, McClain MR, Palomaki GE, Neveux LM, Lambert-Messerlian G, Canick JA, Malone FD, Porter TF, Nyberg DA, Bernstein PS, D'Alton ME; First- and Second-Trimester Risk of Aneuploidy (FaSTER) Research Consortium 2010 Thyroperoxidase and thyroglobulin antibodies in early pregnancy and preterm delivery. Obstet Gynecol 116:58-62.

191. Männistö T, Vääräsmäki M, Pouta A, Hartikainen AL, Ruokonen A, Surcel HM, Bloigu A, Järvelin MR, Suvanto-Luukkonen E 2009 Perinatal outcome of children born to mothers with thyroid dysfunction or antibodies: a prospective population-based cohort study. J Clin Endocrinol Metab 94:772-779.

192. Karakosta P, Alegakis D, Georgiou V, Roumeliotaki T, Fthenou E, Vassilaki M, Boumpas D, Castanas E, Kogevinas M, Chatzi L 2012 Thyroid dysfunction and autoantibodies in early pregnancy are associated with increased risk of gestational diabetes and adverse birth outcomes. J Clin Endocrinol Metab 97:4464-4472.

193. Kumru P, Erdogdu E, Arisoy R, Demirci O, Ozkoral A, Ardic C, Ertekin AA, Erdogan S, Ozdemir NN 2015 Effect of thyroid dysfunction and autoimmunity on pregnancy outcomes in low risk population. Arch Gynecol Obstet 291:1047-1054.

194. Negro R 2011 Thyroid autoimmunity and pre-term delivery: brief review and meta-analysis. J Endocrinol Invest 34:155-158.

195. He X, Wang P, Wang Z, He X, Xu D, Wang B 2012 Thyroid antibodies and risk of preterm delivery: a metaanalysis of prospective cohort studies. Eur J Endocrinol 167:455-464.

196. Negro R, Schwartz A, Gismondi R, Tinelli A, Mangieri T, Stagnaro-Green A 2011 Thyroid antibody positivity in the first trimester of pregnancy is associated with negative pregnancy outcomes. J Clin Endocrinol Metab 96:E920-E924.

197. Groer MW, Vaughan JH 2013 Positive thyroid peroxidase antibody titer is associated with dysphoric moods during pregnancy and postpartum. J Obstet Gynecol Neonatal Nurs 42:E26-E32.

198. Kuijpens JL, Vader HL, Drexhage HA, Wiersinga WM, van Son MJ, Pop VJ 2001 Thyroid peroxidase antibodies during gestation are a marker for subsequent depression postpartum. Eur J Endocrinol 145:579-584.

199. Li Y, Shan Z, Teng W, Yu X, Li Y, Fan C, Teng X, Guo $\mathrm{R}$, Wang H, Li J, Chen Y, Wang W, Chawinga M, Zhang L, Yang L, Zhao Y, Hua T 2010 Abnormalities of maternal thyroid function during pregnancy affect neuropsychological development of their children at 25-30 months. Clin Endocrinol (Oxf) 72:825-829.

200. Williams FL, Watson J, Ogston SA, Visser TJ, Hume R, Willatts P 2013 Maternal and umbilical cord levels of T4, FT4, TSH, TPOAb, and TgAb in term infants and neurodevelopmental outcome at 5.5 years. J Clin Endocrinol Metab 98:829-838.
201. Wasserman EE, Pillion JP, Duggan A, Nelson K, Rohde C, Seaberg EC, Talor MV, Yolken RH, Rose NR 2012 Childhood IQ, hearing loss, and maternal thyroid autoimmunity in the Baltimore Collaborative Perinatal Project. Pediatr Res 72:525-530.

202. Ghassabian A, Bongers-Schokking JJ, de Rijke YB, van Mil N, Jaddoe VW, de Muinck Keizer-Schrama SM, Hooijkaas H, Hofman A, Visser W, Roman GC, Visser TJ, Verhulst FC, Tiemeier H 2012 Maternal thyroid autoimmunity during pregnancy and the risk of attention deficit/ hyperactivity problems in children: the Generation $\mathrm{R}$ Study. Thyroid 22:178-186.

203. Brown AS, Surcel HM, Hinkka-Yli-Salomäki S, Cheslack-Postava K, Bao Y, Sourander A 2015 Maternal thyroid autoantibody and elevated risk of autism in a national birth cohort. Prog Neuropsychopharmacol Biol Psychiatry 57:86-92.

204. Zegers-Hochschild F, G. D. Adamson, J. de Mouzon, O. Ishihara, R. Mansour, K. Nygren, E. Sullivan, and S. Vanderpoel, for ICMART and WHO 2009 International Committee for Monitoring Assisted Reproductive Technology (ICMART) and the World Health Organization (WHO) revised glossary of ART terminology, 2009. Fertil Steril 92:1520-1524.

205. Chandra A, Martinez GM, Mosher WD, Abma JC, Jones J 2005 Fertility, family planning, and reproductive health of U.S. women: data from the 2002 National Survey of Family Growth. Vital Health Stat 23(25):1-160.

206. Krassas GE, Poppe K, Glinoer D 2010 Thyroid function and human reproductive health. Endocr Rev 31:702-755.

207. Krassas GE, Pontikides N, Kaltsas T, Papadopoulou P, Batrinos M 1994 Menstrual disturbances in thyrotoxicosis. Clin Endocrinol (Oxf) 40:641-644.

208. Poppe K, Glinoer D, Van Steirteghem A, Tournaye H, Devroey P, Schiettecatte J, Velkeniers B 2002 Thyroid dysfunction and autoimmunity in infertile women. Thyroid 12:997-1001.

209. Krassas GE, Pontikides N, Kaltsas T, Papadopoulou P, Paunkovic J, Paunkovic N, Duntas LH 1999 Disturbances of menstruation in hypothyroidism. Clin Endocrinol (Oxf) 50:655-659.

210. Grassi G, Balsamo A, Ansaldi C, Balbo A, Massobrio M, Benedetto C 2001Thyroid autoimmunity and infertility. Gynecol Endocrinol 15:389-396.

211. Quintino-Moro A, Zantut-Wittmann DE, Tambascia M, Machado Hda C, Fernandes A 2014 High prevalence of infertility among women with Graves' disease and Hashimoto's thyroiditis. Int J Endocrinol 2014:982705.

212. Lincoln SR, Ke RW, Kutteh WH 1999 Screening for hypothyroidism in infertile women. J Reprod Med 44:455-457.

213. Abalovich M, Mitelberg L, Allami C, Gutierrez S, Alcaraz G, Otero P, Levalle O 2007 Subclinical hypothyroidism and thyroid autoimmunity in women with infertility. Gynecol Endocrinol 23:279-283.

214. Lee YJ, Kim CH, Kwack JY, Ahn JW, Kim SH, Chae HD, Kang BM 2014 Subclinical hypothyroidism diagnosed by thyrotropin-releasing hormone stimulation test in infertile women with basal thyroid-stimulating hormone levels of 2.5 to $5.0 \mathrm{mIU} / \mathrm{L}$. Obstet Gynecol Sci 57:507-512.

215. Arojoki M, Jokimaa V, Juuti A, Koskinen P, Irjala K, Anttila L 2000 Hypothyroidism among infertile women in Finland. Gynecol Endocrinol 14:127-131.

216. Verma I, Sood R, Juneja S, Kaur S 2012 Prevalence of hypothyroidism in infertile women and evaluation of response 
of treatment for hypothyroidism on infertility. Int $\mathbf{J}$ Appl Basic Med Res 2:17-19.

217. Yoshioka W, Amino N, Ide A, Kang S, Kudo T, Nishihara E, Ito M, Nakamura H, Miyauchi A 2015 Thyroxine treatment may be useful for subclinical hypothyroidism in patients with female infertility. Endocr J 62:87-92.

218. Kachuei M, Jafari F, Kachuei A, Keshteli AH 2012 Prevalence of autoimmune thyroiditis in patients with polycystic ovary syndrome. Arch Gynecol Obstet 285:853-856.

219. Monteleone P, Parrini D, Faviana P, Carletti E, Casarosa E, Uccelli A, Cela V, Genazzani AR, Artini PG 2011 Female infertility related to thyroid autoimmunity: the ovarian follicle hypothesis. Am J Reprod Immunol 66: 108-114.

220. Ott J, Aust S, Kurz C, Nouri K, Wirth S, Huber JC, Mayerhofer K 2010 Elevated antithyroid peroxidase antibodies indicating Hashimoto's thyroiditis are associated with the treatment response in infertile women with polycystic ovary syndrome. Fertil Steril 94:2895-2897.

221. Jatzko B, Vytiska-Bistorfer E, Pawlik A, Promberger R, Mayerhofer K, Ott J. 2014 The impact of thyroid function on intrauterine insemination outcome-a retrospective analysis. Reprod Biol Endocrinol 12:28.

222. Karmon AE, Batsis M, Chavarro JE, Souter I 2015 Preconceptional thyroid-stimulating hormone levels and outcomes of intrauterine insemination among euthyroid infertile women. Fertil Steril 103:258-263.

223. Baker VL, Rone HM, Pasta DJ, Nelson HP, Gvakharia M, Adamson GD 2006 Correlation of thyroid stimulating hormone (TSH) level with pregnancy outcome in women undergoing in vitro fertilization. Am J Obstet Gynecol 194: 1668-1674.

224. Fumarola A, Grani G, Romanzi D, Del Sordo M, Bianchini M, Aragona A, Tranquilli D, Aragona C 2013 Thyroid function in infertile patients undergoing assisted reproduction. Am J Reprod Immunol 70:336-341.

225. Reh A, Grifo J, Danoff A 2010 What is a normal thyroidstimulating hormone (TSH) level? Effects of stricter TSH thresholds on pregnancy outcomes after in vitro fertilization. Fertil Steril 94:2920-2922.

226. Chai J, Yeung WY, Lee CY, Li HW, Ho PC, Ng HY 2014 Live birth rates following in vitro fertilization in women with thyroid autoimmunity and/or subclinical hypothyroidism. Clin Endocrinol (Oxf) 80:122-127.

227. Weghofer A, Himaya E, Kushnir VA, Barad DH, Gleicher N 2015 The impact of thyroid function and thyroid autoimmunity on embryo quality in women with low functional ovarian reserve: a case-control study. Reprod Biol Endocrinol 13:43.

228. Aghahosseini M, Asgharifard H, Aleyasin A, Tehrani Banihashemi A 2014 Effects of thyroid stimulating hormone (TSH) level on clinical pregnancy rate via in vitro fertilization (IVF) procedure. Med J Islam Repub Iran 28:46.

229. Green KA, Werner MD, Franasiak JM, Juneau CR, Hong KH, Scott RT Jr 2015 Investigating the optimal preconception TSH range for patients undergoing IVF when controlling for embryo quality. J Assist Reprod Genet 32: 1469-1476.

230. Abdel Rahman AH, Aly Abbassy H, Abbassy AA 2010 Improved in vitro fertilization outcomes after treatment of subclinical hypothyroidism in infertile women. Endocr Pract 16:792-797.

231. Kim CH, Ahn JW, Kang SP, Kim SH, Chae HD, Kang BM 2011 Effect of levothyroxine treatment on in vitro fertilization and pregnancy outcome in infertile women with subclinical hypothyroidism undergoing in vitro fertilization/intracytoplasmic sperm injection. Fertil Steril 95:1650-1654.

232. Negro R, Mangieri T, Coppola L, Presicce G, Casavola EC, Gismondi R, Locorotondo G, Caroli P, Pezzarossa A, Dazzi D, Hassan H 2005 Levothyroxine treatment in thyroid peroxidase antibody-positive women undergoing assisted reproduction technologies: a prospective study. Hum Reprod 20:1529-1533.

233. Velkeniers B, Van Meerhaeghe A, Poppe K, Unuane D, Tournaye H, Haentjens P 2013 Levothyroxine treatment and pregnancy outcome in women with subclinical hypothyroidism undergoing assisted reproduction technologies: systematic review and meta-analysis of RCTs. Hum Reprod Update 19:251-258.

234. Karmisholt J, Andersen S, Laurberg P 2011 Variation in thyroid function in subclinical hypothyroidism: importance of clinical follow-up and therapy. Eur J Endocrinol 164:317-323.

235. Scoccia B, Demir H, Kang Y, Fierro MA, Winston NJ 2012 In vitro fertilization pregnancy rates in levothyroxine-treated women with hypothyroidism compared to women without thyroid dysfunction disorders. Thyroid 22:631-636.

236. Busnelli A, Somigliana E, Benaglia L, Leonardi M, Ragni G, Fedele L 2013 In vitro fertilization outcomes in treated hypothyroidism. Thyroid 23:1319-1325.

237. Toulis KA, Goulis DG, Venetis CA, Kolibianakis EM, Negro R, Tarlatzis BC, Papadimas I 2010 Risk of spontaneous miscarriage in euthyroid women with thyroid autoimmunity undergoing IVF: a meta-analysis. Eur J Endocrinol 162:643-652.

238. Tan S, Dieterle S, Pechlavanis S, Janssen OE, Fuhrer D 2014Thyroid autoantibodies per se do not impair intracytoplasmic sperm injection outcome in euthyroid healthy women. Eur J Endocrinol 170:495-500.

239. Łukaszuk K, Kunicki M, Kulwikowska P, Liss J, Pastuszek E, Jaszczołt M, Męczekalski B, Skowroński K 2015 The impact of the presence of antithyroid antibodies on pregnancy outcome following intracytoplasmatic sperm injection-ICSI and embryo transfer in women with normal thyreotropine levels. J Endocrinol Invest 38:1335-1343.

240. Karacan M, Alwaeely F, Cebi Z, Berberoglugil M, Batukan M, Ulug M, Arvas A, Camlibel T 2013 Effect of antithyroid antibodies on ICSI outcome in antiphospholipid antibody-negative euthyroid women. Reprod Biomed Online 27:376-380.

241. Zhong YP, Ying Y, Wu HT, Zhou CQ, Xu YW, Wang Q, Li J, Shen XT, Li J 2012 Relationship between antithyroid antibody and pregnancy outcome following in vitro fertilization and embryo transfer. Int J Med Sci 9:121-125.

242. Revelli A, Casano S, Piane LD, Grassi G, Gennarelli G, Guidetti D, Massobrio M 2009 A retrospective study on IVF outcome in euthyroid patients with anti-thyroid antibodies: effects of levothyroxine, acetyl-salicylic acid and prednisolone adjuvant treatments. Reprod Biol Endocrinol 7:137.

243. Litwicka K, Arrivi C, Varricchio MT, Mencacci C, Greco E 2015 In women with thyroid autoimmunity, does lowdose prednisolone administration, compared with no adjuvant therapy, improve in vitro fertilization clinical results? J Obstet Gynaecol Res 41:722-728.

244. Turi A, Giannubilo SR, Zanconi S, Mascetti A, Tranquilli AL 2010 Preconception steroid treatment in infertile women 
with antithyroid autoimmunity undergoing ovarian stimulation and intrauterine insemination: a double-blind, randomized, prospective cohort study. Clin Ther 32:2415-2421.

245. Michael AE, Papageorghiou AT 2008 Potential significance of physiological and pharmacological glucocorticoids in early pregnancy. Hum Reprod Update 14:497-517.

246. Poppe K, Unuane D, D'Haeseleer M, Tournaye H, Schiettecatte J, Haentjens P, Velkeniers B 2011 Thyroid function after controlled ovarian hyperstimulation in women with and without the hyperstimulation syndrome. Fertil Steril 96:241-245.

247. Mintziori G, Goulis DG, Toulis KA, Venetis CA, Kolibianakis EM, Tarlatzis BC 2011 Thyroid function during ovarian stimulation: a systematic review. Fertil Steril 96:780-785.

248. Muller AF, Verhoeff A, Mantel MJ, De Jong FH, Berghout A 2000 Decrease of free thyroxine levels after controlled ovarian hyperstimulation. J Clin Endocrinol Metab 85:545-548.

249. Poppe K, Glinoer D, Tournaye H, Schiettecatte J, Devroey P, van Steirteghem A, Haentjens P, Velkeniers B 2004 Impact of ovarian hyperstimulation on thyroid function in women with and without thyroid autoimmunity. J Clin Endocrinol Metab 89:3808-3812.

250. Poppe K, Glinoer D, Tournaye H, Schiettecatte J, Haentjens P, Velkeniers B 2005 Thyroid function after assisted reproductive technology in women free of thyroid disease. Fertil Steril 83:1753-1757.

251. Gracia CR, Morse CB, Chan G, Schilling S, Prewitt M, Sammel MD, Mandel SJ 2012 Thyroid function during controlled ovarian hyperstimulation as part of in vitro fertilization. Fertil Steril 97:585-591.

252. Reinblatt S, Herrero B, Correa JA, Shalom-Paz E, Ata B, Wiser A, Morris D, Holzer H 2013 Thyroid stimulating hormone levels rise after assisted reproductive technology. J Assist Reprod Genet 30:1347-1352.

253. Corbett S, Shmorgun D, Claman P, Reproductive Endocrinology Infertility Committee, Healey S, Gysler M 2014 The prevention of ovarian hyperstimulation syndrome. J Obstet Gynaecol Can 36:1024-1036.

254. Skweres T, Wójcik D, Ciepłuch R, Sliwiński W, Czech R, Gruszczyński W, Rogacki M 2014 Thyroid dysfunction during severe ovarian hyperstimulation syndrome. A case report. Ginekol Pol 85:472-475.

255. Stuckey BG, Yeap D, Turner SR 2010 Thyroxine replacement during super-ovulation for in vitro fertilization: a potential gap in management? Fertil Steril 93:2414.e1-3.

256. Busnelli A, Somigliana E, Benaglia L, Sarais V, Ragni G, Fedele L 2014 Thyroid axis dysregulation during in vitro fertilization in hypothyroid-treated patients. Thyroid 24:1650-1655.

257. Busnelli A, Vannucchi G, Paffoni A, Faulisi S, Fugazzola L, Fedele L, Somigliana E 2015 Levothyroxine dose adjustment in hypothyroid women achieving pregnancy through IVF. Eur J Endocrinol 173:417-424.

258. Davis LB, Lathi RB, Dahan MH 2007 The effect of infertility medication on thyroid function in hypothyroid women who conceive. Thyroid 17:773-777.

259. Casey BM, Dashe JS, Wells CE, McIntire DD, Byrd W, Leveno KJ, Cunningham FG 2005 Subclinical hypothyroidism and pregnancy outcomes. Obstet Gynecol 105: 239-245.

260. Allan WC, Haddow JE, Palomaki GE, Williams JR, Mitchell ML, Hermos RJ, Faix JD, Klein RZ 2000 Ma- ternal thyroid deficiency and pregnancy complications: implications for population screening. J Med Screen 7:127-130.

261. Casey BM, Dashe JS, Spong CY, McIntire DD, Leveno KJ, Cunningham GF 2007 Perinatal significance of isolated maternal hypothyroxinemia identified in the first half of pregnancy. Obstet Gynecol 109:1129-1135.

262. Stricker R, Echenard M, Eberhart R, Chevailler MC, Perez V, Quinn FA, Stricker R 2007 Evaluation of maternal thyroid function during pregnancy: the importance of using gestational age-specific reference intervals. Eur J Endocrinol 157:509-514.

263. Soldin OP, Soldin D, Sastoque M 2007 Gestation-specific thyroxine and thyroid stimulating hormone levels in the United States and worldwide. Ther Drug Monit 29:553-559.

264. Bocos-Terraz JP, Izquierdo-Alvarez S, Bancalero-Flores JL, Alvarez-Lahuerta R, Aznar-Sauca A, Real-Lopez E, Ibanez-Marco R, Bocanegra-Garcia V, Rivera-Sanchez G 2009 Thyroid hormones according to gestational age in pregnant Spanish women. BMC Res Notes 2:237.

265. La'ulu SL, Roberts WL 2011 Ethnic differences in first trimester thyroid reference intervals. Clin Chem 57:913-915.

266. Mannisto T, Surcel HM, Ruokonen A, Vaarasmaki M, Pouta A, Bloigu A, Järvelin MR, Hartikainen AL, Suvanto E 2011 Early pregnancy reference intervals of thyroid hormone concentrations in a thyroid antibody-negative pregnant population. Thyroid 21:291-298.

267. Medici M, de Rijke YB, Peeters RP, Visser W, de Muinck Keizer-Schrama SM, Jaddoe VV, Hofman A, Hooijkaas $\mathrm{H}$, Steegers EA, Tiemeier H, Bongers-Schokking JJ, Visser TJ 2011 Maternal early pregnancy and newborn thyroid hormone parameters: the Generation R study. J Clin Endocrinol Metab 97:646-652.

268. Springer D, Zima T, Limanova Z 2009 Reference intervals in evaluation of maternal thyroid function during the first trimester of pregnancy. Eur J Endocrinol 160:791-797.

269. Medici M, Korevaar TI, Visser WE, Visser TJ, Peeters RP 2015 Thyroid function in pregnancy: what is normal? Clin Chem 61:704-713.

270. Lambert-Messerlian G, McClain M, Haddow JE, Palomaki GE, Canick JA, Cleary-Goldman J, Malone FD, Porter TF, Nyberg DA, Bernstein P, D'Alton ME; FaSTER Research Consortium 2008 First- and secondtrimester thyroid hormone reference data in pregnant women: a FaSTER (First- and Second-Trimester Evaluation of Risk for aneuploidy) Research Consortium study. Am J Obstet Gynecol 199:62.e1-62.e6.

271. Gilbert RM, Hadlow NC, Walsh JP, Fletcher SJ, Brown SJ, Stuckey BG, Lim EM 2008 Assessment of thyroid function during pregnancy: first-trimester (weeks 9-13) reference intervals derived from Western Australian women. Med J Aust 189:250-253.

272. Quinn FA, Gridasov GN, Vdovenko SA, Krasnova NA, Vodopianova NV, Epiphanova MA, Schulten M 2005 Prevalence of abnormal thyroid stimulating hormone and thyroid peroxidase antibody-positive results in a population of pregnant women in the Samara region of the Russian Federation. Clin Chem Lab Med 43:1223-1226.

273. Vaidya B, Hubalewska-Dydejczyk A, Laurberg P, Negro R, Vermiglio F, Poppe K 2012 Treatment and screening of hypothyroidism in pregnancy: results of a European survey. Eur J Endocrinol 166:49-54.

274. Vaidya B, Anthony S, Bilous M, Shields B, Drury J, Hutchison S, Bilous R 2007 Detection of thyroid dysfunction 
in early pregnancy: universal screening or targeted high-risk case findings? J Clin Endocrinol Metab 92:203-207.

275. van den Boogaard E, Vissenberg R, Land JA, van Wely M, van der Post JA, Goddijn M, Bisschop PH 2011 Significance of (sub)clinical thyroid dysfunction and thyroid autoimmunity before conception and in early pregnancy: a systematic review. Hum Reprod Update 17:605-619.

276. Haddow JE, Palomaki GE, Allan WC, Williams JR, Knight GJ, Gagnon J, O'Heir CE, Mitchell ML, Hermos RJ, Waisbren SE, Faix JD, Klein RZ 1999 Maternal thyroid deficiency during pregnancy and subsequent neuropsychological development of the child. N Engl J Med 341:549-555.

277. Abalovich M, Gutierrez S, Alcaraz G, Maccallini G, Garcia A, Levalle O 2002 Overt and subclinical hypothyroidism complicating pregnancy. Thyroid 12:63-68.

278. Leung AS, Millar LK, Koonings PP, Montoro M, Mestman JH 1993 Perinatal outcome in hypothyroid pregnancies. Obstet Gynecol 81:349-353.

279. Sahu MT, Das V, Mittal S, Agarwal A, Sahu M 2010 Overt and subclinical thyroid dysfunction among Indian pregnant women and its effect on maternal and fetal outcome. Arch Gynecol Obstet 281:215-220.

280. Ong GS, Hadlow NC, Brown SJ, Lim EM, Walsh JP 2014 Does the thyroid-stimulating hormone measured concurrently with first trimester biochemical screening tests predict adverse pregnancy outcomes occurring after 20 weeks gestation? J Clin Endocrinol Metab 99:E2668E2672.

281. Chen LM, Du WJ, Dai J, Zhang Q, Si GX, Yang H, Ye EL, Chen QS, Yu LC, Zhang C, Lu XM 2014 Effects of subclinical hypothyroidism on maternal and perinatal outcomes during pregnancy: a single-center cohort study of a Chinese population. PLoS One 9(10): e109364.

282. Godoy GA, Korevaar TI, Peeters RP, Hofman A, de Rijke YB, Bongers-Schokking JJ, Tiemeier H, Jaddoe VW, Gaillard R 2014 Maternal thyroid hormones during pregnancy, childhood adiposity and cardiovascular risk factors: the Generation R Study. Clin Endocrinol (Oxf) 81:117-125.

283. León G, Murcia M, Rebagliato M, Álvarez-Pedrerol M, Castilla AM, Basterrechea M, Iñiguez C, FernándezSomoano A, Blarduni E, Foradada CM, Tardón A, Vioque J 2015 Maternal thyroid dysfunction during gestation, preterm delivery, and birthweight. The Infancia y Medio Ambiente Cohort, Spain. Paediatr Perinat Epidemiol 29:113-122.

284. Päkkilä F, Männistö T, Pouta A, Hartikainen AL, Ruokonen A, Surcel HM, Bloigu A, Vääräsmäki M, Järvelin MR, Moilanen I, Suvanto E 2014 The impact of gestational thyroid hormone concentrations on ADHD symptoms of the child. J Clin Endocrinol Metab 99:E1-E8.

285. Medici M, Korevaar T, Schalekamp-Timmermans S, Gaillard R, de Rijke YB, Visser WE, Visser W, de Muinck Keizer-Schrama SM, Hofman A, Hooijkaas H, Bongers-Schokking JJ, Tiemeier H, Jaddoe VW, Visser TJ, Peeters RP, Steegers EA 2014 Maternal earlypregnancy thyroid function is associated with subsequent hypertensive disorders of pregnancy: the generation $\mathrm{R}$ study. J Clin Endocrinol Metab 99:E2591-E2598.

286. Negro R, Schwartz A, Gismondi R, Tinelli A, Mangieri T, Stagnaro-Green A 2010 Increased pregnancy loss rate in thyroid antibody negative women with TSH levels between 2.5 and 5.0 in the first trimester of pregnancy. J Clin Endocrinol Metab 95:E44-E48.
287. Benhadi N, Wiersinga WM, Reitsma JB, Vrijkotte TG, Bonsel GJ 2009 Higher maternal TSH levels in pregnancy are associated with increased risk for miscarriage, fetal or neonatal death. Eur J Endocrinol 160:985-991.

288. Liu H, Shan Z, Li C, Mao J, Xie X, Wang W, Fan C, Wang H, Zhang H, Han C, Wang X, Liu X, Fan Y, Bao S, Teng W 2014 Maternal subclinical hypothyroidism, thyroid autoimmunity, and the risk of miscarriage: a prospective cohort study. Thyroid 24:1642-1649.

289. Cleary-Goldman J, Malone FD, Lambert-Messerlian G, Sullivan L, Canick J, Porter TF, Luthy D, Gross S, Bianchi DW, D'Alton ME 2008 Maternal thyroid hypofunction and pregnancy outcome. Obstet Gynecol 112:85-92.

290. Stagnaro-Green A, Chen X, Bogden JD, Davies TF, Scholl TO 2005 The thyroid and pregnancy: a novel risk factor for very preterm delivery. Thyroid 15:351-357.

291. Su PY, Huang K, Hao JH, Xu YQ, Yan SQ, Li T, Xu YH, Tao FB 2011 Maternal thyroid function in the first twenty weeks of pregnancy and subsequent fetal and infant development: a prospective population-based cohort study in China. J Clin Endocrinol Metab 96:3234-3241.

292. Schneuer FJ, Nassar N, Tasevski V, Morris JM, Roberts CL 2012 Association and predictive accuracy of high TSH serum levels in first trimester and adverse pregnancy outcomes. J Clin Endocrinol Metab 97:3115-3122.

293. Wilson KL, Casey BM, McIntire DD, Halvorson LM, Cunningham FG 2012 Subclinical thyroid disease and the incidence of hypertension in pregnancy. Obstet Gynecol 119:315-320.

294. Mannisto T, Vaarasmaki M, Pouta A, Hartikainen AL, Ruokonen A, Surcel HM, Bloigu A, Jarvelin MR, Suvanto E 2010 Thyroid dysfunction and autoantibodies during pregnancy as predictive factors of pregnancy complications and maternal morbidity in later life. J Clin Endocrinol Metab 95:1084-1094.

295. Mannisto T, Vaarasmaki M, Pouta A, Hartikainen AL, Ruokonen A, Surcel HM, Bloigu A, Jarvelin MR, Suvanto-Luukkonen E 2009 Perinatal outcome of children born to mothers with thyroid dysfunction or antibodies: a prospective population-based cohort study. J Clin Endocrinol Metab 94:772-779.

296. Chan S, Boelaert K 2015 Optimal management of hypothyroidism, hypothyroxinaemia and euthyroid TPO antibody positivity preconception and in pregnancy. Clin Endocrinol (Oxf) 82:313-326.

297. Henrichs J, Bongers-Schokking JJ, Schenk JJ, Ghassabian A, Schmidt HG, Visser TJ, Hooijkaas H, de Muinck Keizer-Schrama SM, Hofman A, Jaddoe VV, Visser W, Steegers EA, Verhulst FC, de Rijke YB, Tiemeier H 2010 Maternal thyroid function during early pregnancy and cognitive functioning in early childhood: the Generation $\mathrm{R}$ study. J Clin Endocrinol Metab 95:4227-4234.

298. Craig WY, Allan WC, Kloza EM, Pulkkinen AJ, Waisbren S, Spratt DI, Palomaki GE, Neveux LM, Haddow JE 2012 Mid-gestational maternal free thyroxine concentration and offspring neurocognitive development at age two years. J Clin Endocrinol Metab 97:E22-E28.

299. Román GC, Ghassabian A, Bongers-Schokking JJ, Jaddoe VW, Hofman A, de Rijke YB, Verhulst FC, Tiemeier H 2013 Association of gestational maternal hypothyroxinemia and increased autism risk. Ann Neurol 74:733-742.

300. Finken MJ, van Eijsden M, Loomans EM, Vrijkotte TG, Rotteveel J 2013 Maternal hypothyroxinemia in early pregnancy predicts reduced performance in reaction time 
tests in 5- to 6-year-old offspring. J Clin Endocrinol Metab 98:1417-1426.

301. Julvez J, Alvarez-Pedrerol M, Rebagliato M, Murcia M, Forns J, Garcia-Esteban R, Lertxundi N, Espada M, Tardón A, Riaño Galán I, Sunyer J 2013 Thyroxine levels during pregnancy in healthy women and early child neurodevelopment. Epidemiology 24:150-157.

302. Ghassabian AE, Marroun H, Peeters RP, Jaddoe VW, Hofman A, Verhulst FC, Tiemeier H, White T 2014 Downstream effects of maternal hypothyroxinemia in early pregnancy: nonverbal IQ and brain morphology in schoolage children. J Clin Endocrinol Metab 99:2383-2390.

303. Gyllenberg D, Sourander A, Surcel HM, Hinkka-YliSalomäki S, McKeague IW, Brown AS 2016 Hypothyroxinemia during gestation and offspring schizophrenia in a national birth cohort. Biol Psychiatry 79:962-970.

304. Modesto T, Tiemeier H, Peeters RP, Jaddoe VW, Hofman A, Verhulst FC, Ghassabian A 2015 Maternal mild thyroid hormone insufficiency in early pregnancy and attentiondeficit/hyperactivity disorder symptoms in children. JAMA Pediatr 169:838-845.

305. Pop VJ, de Vries E, van Baar AL, Waelkens JJ, de Rooy HA, Horsten M, Donkers MM, Komproe IH, van Son MM, Vader HL 1995 Maternal thyroid peroxidase antibodies during pregnancy: a marker of impaired child development? J Clin Endocrinol Metab 80:3561-3566.

306. Lazarus JH, Bestwick JP, Channon S, Paradice R, Maina A, Rees R, Chiusano E, John R, Guaraldo V, George LM, Perona M, Dall'Amico D, Parkes AB, Joomun M, Wald NJ 2012 Antenatal thyroid screening and childhood cognitive function. N Engl J Med 366:493-501.

307. Liu H, Momotani N, Noh JY, Ishikawa N, Takebe K, Ito K 1994 Maternal hypothyroidism during early pregnancy and intellectual development of the progeny. Arch Intern Med 154:785-787.

308. Momotani N, Iwama S, Momotani K 2012 Neurodevelopment in children born to hypothyroid mothers restored to normal thyroxine (T4) concentration by late pregnancy in Japan: no apparent influence of maternal T4 deficiency. J Clin Endocrinol Metabol 97:1104-1108.

309. Downing S, Halpern L, Carswell J, Brown RS 2012 Severe maternal hypothyroidism corrected prior to the third trimester is associated with normal cognitive outcome in the offspring. Thyroid 22:625-630.

310. Ausó E, Lavado-Autric R, Cuevas E, Del Rey FE, Morreale De Escobar G, Berbel P 2004 A moderate and transient deficiency of maternal thyroid function at the beginning of fetal neocorticogenesis alters neuronal migration. Endocrinol 145:4037-4047.

311. Wang S, Teng W, Gao Y, Fan C, Zhang H, Shan Z 2012 Early levothyroxine treatment on maternal subclinical hypothyroidism improves spatial learning of offspring in rats. J Neuroendocrinol 24:841-848.

312. Pop VJ, Brouwers EP, Vader HL, Vulsma T, van Baar AL, de Vijlder JJ 2003 Maternal hypothyroxinaemia during early pregnancy and subsequent child development: a 3-year follow-up study. Clin Endocrinol (Oxf) 59:282-288.

313. Henrichs J, Ghassabian A, Peeters RP, Tiemeier H 2013 Maternal hypothyroxinemia and effects on cognitive functioning in childhood: how and why? Clin Endocrinol (Oxf) 79:152-162.

314. Medici M, Timmermans S, Visser W, de Muinck KeizerSchrama SM, Jaddoe VW, Hofman A, Hooijkaas H, de
Rijke YB, Tiemeier H, Bongers-Schokking JJ, Visser TJ, Peeters RP, Steegers EA 2013 Maternal thyroid hormone parameters during early pregnancy and birth weight: the Generation R Study. J Clin Endocrinol Metab 98:59-66.

315. León G, Murcia M, Rebagliato M, Álvarez-Pedrerol M, Castilla AM, Basterrechea M, Iñiguez C, FernándezSomoano A, Blarduni E, Foradada CM, Tardón A, Vioque J 2015 Maternal thyroid dysfunction during gestation, preterm delivery, and birthweight. The Infancia y Medio Ambiente Cohort, Spain. Paediatr Perinat Epidemiol 29:113-122.

316. Haddow JE, Craig WY, Neveux LM, Haddow HR, Palomaki GE, Lambert-Messerlian G, Malone FD, D'Alton ME, First and Second Trimester Risk of Aneuploidy (FaSTER) Research Consortium 2014 Implications of high free thyroxine (FT4) concentrations in euthyroid pregnancies: the FaSTER trial. J Clin Endocrinol Metab 99:2038-2044.

317. Korevaar TI, Muetzel R, Medici M, Chaker L, Jaddoe VW, de Rijke YB, Steegers EA, Visser TJ, White T, Tiemeier H, Peeters RP 2016 Association of maternal thyroid function during early pregnancy with offspring IQ and brain morphology in childhood: a population-based prospective cohort study. Lancet Diabetes Endocrinol 4:35-43.

318. Taylor PN, Minassian C, Rehman A, Iqbal A, Draman MS, Hamilton W, Dunlop D, Robinson A, Vaidya B, Lazarus JH, Thomas S, Dayan CM, Okosieme OE 2014 TSH levels and risk of miscarriage in women on longterm levothyroxine: a community-based study. J Clin Endocrinol Metab 99:3895-3902.

319. Lev-Ran A 1983 Part-of-the-day hypertriiodothyroninemia caused by desiccated thyroid. JAMA 250:2790-2791.

320. Wiersinga W, Duntas L, Fadeyev V, Nygaard B, Vanderpump M 20122012 ETA guidelines: the use of L-T4 + L-T3 in the treatment of hypothyroidism. Eur Thyroid J 1:55-71.

321. Alexander EK, Marqusee E, Lawrence J, Jarolim P, Fischer GA, Larsen PR 2004 Timing and magnitude of increases in levothyroxine requirements during pregnancy in women with hypothyroidism. N Engl J Med 351:241-249.

322. Yassa L, Marqusee E, Fawcett R, Alexander EK 2010 Thyroid hormone early adjustment in pregnancy (the THERAPY) trial. J Clin Endocrinol Metab 95:3234-3241.

323. Mandel SJ, Larsen PR, Seely EW, Brent GA 1990 Increased need for thyroxine during pregnancy in women with primary hypothyroidism. N Engl J Med 323:91-96.

324. Kaplan MM 1992 Monitoring thyroxine treatment during pregnancy. Thyroid 2:147-152.

325. Loh JA, Wartofsky L, Jonklaas J, Burman KD 2009 The magnitude of increased levothyroxine requirements in hypothyroid pregnant women depends upon the etiology of the hypothyroidism. Thyroid 19:269-275.

326. Abalovich M, Alcaraz G, Kleiman-Rubinsztein J, Pavlove MM, Cornelio C, Levalle O, Gutierrez S 2010 The relationship of preconception thyrotropin levels to requirements for increasing the levothyroxine dose during pregnancy in women with primary hypothyroidism. Thyroid 20:1175-1178.

327. Galofre JC, Haber RS, Mitchell AA, Pessah R, Davies TF. 2010 Increased postpartum thyroxine replacement in Hashimoto's thyroiditis. Thyroid 20:901-908.

328. Cooper DS, Laurberg P 2013 Hyperthyroidism in pregnancy. Lancet Diabetes Endocrinol 1:238-249. 
329. Bolz M, Korber S, Schober HC 2013 TSH secreting adenoma of pituitary gland (TSHom) - rare cause of hyperthyroidism in pregnancy. Dtsch Med Wochenschr 138:362-366.

330. Merza Z, White D, Khanem N 2015 Struma ovarii in pregnancy: an uncommon cause of hyperthyroidism. Clin Nucl Med 40:687-688.

331. Paschke R, Niedziela M, Vaidya B, Persani L, Rapoport B, Leclere J 20122012 European Thyroid Association guidelines for the management of familial and persistent sporadic non-autoimmune hyperthyroidism caused by thyroid-stimulating hormone receptor germline mutations. Eur Thyroid J 1:142-147.

332. Goodwin TM, Montoro M, Mestman JH 1992 Transient hyperthyroidism and hyperemesis gravidarum: clinical aspects. Am J Obstet Gynecol 167:648-652.

333. Tan JY, Loh KC, Yeo GS, Chee YC 2002 Transient hyperthyroidism of hyperemesis gravidarum. BJOG 109:683-688.

334. Niebyl JR 2010 Clinical practice. Nausea and vomiting in pregnancy. N Engl J Med 363:1544-1550.

335. Verberg MF, Gillott DJ, Al-Fardan N, Grudzinskas JG 2005 Hyperemesis gravidarum, a literature review. Hum Reprod Update 11:527-539.

336. Hershman JM 1999 Human chorionic gonadotropin and the thyroid: hyperemesis gravidarum and trophoblastic tumors. Thyroid 9:653-657.

337. Grun JP, Meuris S, De Nayer P, Glinoer D 1997 The thyrotrophic role of human chorionic gonadotrophin (hCG) in the early stages of twin (versus single) pregnancies. Clin Endocrinol (Oxf) 46:719-725.

338. Rodien P, Bremont C, Sanson ML, Parma J, Van Sande J, Costagliola S, Luton JP, Vassart G, Duprez L 1998 Familial gestational hyperthyroidism caused by a mutant thyrotropin receptor hypersensitive to human chorionic gonadotropin. N Engl J Med 339:1823-1826.

339. Glinoer D, Spencer CA 2010 Serum TSH determinations in pregnancy: how, when and why? Nat Rev Endocrinol 6:526-529.

340. Yoshihara A, Noh JY, Mukasa K, Suzuki M, Ohye H, Matsumoto M, Kunii Y, Watanabe N, Suzuki N, Kameda T, Sugino K, Ito K 2015 Serum human chorionic gonadotropin levels and thyroid hormone levels in gestational transient thyrotoxicosis: is the serum hCG level useful for differentiating between active Graves' disease and GTT? Endocr J 62:557-560.

341. Carle A, Knudsen N, Pedersen IB, Perrild H, Ovesen L, Rasmussen LB, Laurberg P 2013 Determinants of serum $\mathrm{T} 4$ and T3 at the time of diagnosis in nosological types of thyrotoxicosis: a population-based study. Eur J Endocrinol 169:537-545.

342. Bouillon R, Naesens M, Van Assche FA, De Keyser L, De Moor P, Renaer M, De Vos P, De Roo M 1982 Thyroid function in patients with hyperemesis gravidarum. Am $\mathrm{J}$ Obstet Gynecol 143:922-926.

343. Ross DS, Burch HB, Cooper DS, Greenlee MC, Laurberg P, Maia AL, Rivkees SA, Samuels M, Sosa JA, Stan MN, Walter MA 20162016 American Thyroid Association guidelines for diagnosis and management of hyperthyroidism and other causes of thyrotoxicosis. Thyroid 26:1343-1421.

344. Laurberg P, Bournaud C, Karmisholt J, Orgiazzi J 2009 Management of Graves' hyperthyroidism in pregnancy: focus on both maternal and foetal thyroid function, and caution against surgical thyroidectomy in pregnancy. Eur J Endocrinol 160:1-8.

345. Alexander EK, Larsen PR 2002 High dose of (131)I therapy for the treatment of hyperthyroidism caused by Graves' disease. J Clin Endocrinol Metab 87:1073-1077.

346. Moura-Neto A, Mosci C, Santos AO, Amorim BJ, de Lima MC, Etchebehere EC, Tambascia MA, Ramos CD, Zantut-Wittmann DE 2012 Predictive factors of failure in a fixed $15 \mathrm{mCi}{ }^{131}$ I-iodide therapy for Graves' disease. Clin Nucl Med 37:550-554.

347. de Jong JA, Verkooijen HM, Valk GD, Zelissen PM, de Keizer B 2013 High failure rates after (131)I therapy in Graves hyperthyroidism patients with large thyroid volumes, high iodine uptake, and high iodine turnover. Clin Nucl Med 38:401-406.

348. Schneider DF, Sonderman PE, Jones MF, Ojomo KA, Chen H, Jaume JC, Elson DF, Perlman SB, Sippel RS 2014 Failure of radioactive iodine in the treatment of hyperthyroidism. Ann Surg Oncol 21:4174-4180.

349. Davis LE, Lucas MJ, Hankins GD, Roark ML, Cunningham FG 1989 Thyrotoxicosis complicating pregnancy. Am J Obstet Gynecol 160:63-70.

350. Millar LK, Wing DA, Leung AS, Koonings PP, Montoro MN, Mestman JH 1994 Low birth weight and preeclampsia in pregnancies complicated by hyperthyroidism. Obstet Gynecol 84:946-949.

351. Andersen SL, Olsen J, Laurberg P 2015 Foetal programming by maternal thyroid disease. Clin Endocrinol (Oxf) 83:751-758.

352. Mandel SJ, Cooper DS 2001 The use of antithyroid drugs in pregnancy and lactation. J Clin Endocrinol Metab 86:2354-2359.

353. Azizi F 2006 The safety and efficacy of antithyroid drugs. Expert Opin Drug Saf 5:107-116.

354. Nicholas WC, Fischer RG, Stevenson RA, Bass JD 1995 Single daily dose of methimazole compared to every 8 hours propylthiouracil in the treatment of hyperthyroidism. South Med J 88:973-976.

355. Kallner G, Vitols S, Ljunggren JG 1996 Comparison of standardized initial doses of two antithyroid drugs in the treatment of Graves' disease. J Intern Med 239:525-529.

356. Nakamura H, Noh JY, Itoh K, Fukata S, Miyauchi A, Hamada N 2007 Comparison of methimazole and propylthiouracil in patients with hyperthyroidism caused by Graves' disease. J Clin Endocrinol Metab 92:2157-2162.

357. Homsanit M, Sriussadaporn S, Vannasaeng S, Peerapatdit T, Nitiyanant W, Vichayanrat A 2001 Efficacy of single daily dosage of methimazole vs. propylthiouracil in the induction of euthyroidism. Clin Endocrinol (Oxf) 54:385-390.

358. He CT, Hsieh AT, Pei D, Hung YJ, Wu LY, Yang TC, Lian WC, Huang WS, Kuo SW 2004 Comparison of single daily dose of methimazole and propylthiouracil in the treatment of Graves' hyperthyroidism. Clin Endocrinol (Oxf) 60:676-681.

359. Nakamura H, Miyauchi A, Miyawaki N, Imagawa J 2013 Analysis of 754 cases of antithyroid drug-induced agranulocytosis over 30 years in Japan. J Clin Endocrinol Metab 98:4776-4783.

360. Watanabe N, Narimatsu H, Noh JY, Yamaguchi T, Kobayashi K, Kami M, Kunii Y, Mukasa K, Ito K, Ito K 2012 Antithyroid drug-induced hematopoietic damage: a retrospective cohort study of agranulocytosis and pancytopenia involving 50,385 patients with Graves' disease. J Clin Endocrinol Metab 97:E49-E53. 
361. Kobayashi S, Noh JY, Mukasa K, Kunii Y, Watanabe N, Matsumoto M, Ohye H, Suzuki M, Yoshihara A, Iwaku K, Sugino K, Ito K 2014 Characteristics of agranulocytosis as an adverse effect of antithyroid drugs in the second or later course of treatment. Thyroid 24:796-801.

362. Rivkees SA, Mattison DR 2009 Propylthiouracil (PTU) hepatoxicity in children and recommendations for discontinuation of use. Int J Pediatr Endocrinol 2009:132041.

363. Russo MW, Galanko JA, Shrestha R, Fried MW, Watkins P 2004 Liver transplantation for acute liver failure from drug induced liver injury in the United States. Liver Transpl 10:1018-1023.

364. Bahn RS, Burch HS, Cooper DS, Garber JR, Greenlee CM, Klein IL, Laurberg P, McDougall IR, Rivkees SA, Ross D, Sosa JA, Stan MN 2009 The role of propylthiouracil in the management of Graves' disease in adults: report of a meeting jointly sponsored by the American Thyroid Association and the Food and Drug Administration. Thyroid 19:673-674.

365. Anderson SL, Olsen J, Laurberg P 2016 Antithyroid drug side effects in the population and in pregnancy. J Clin Endocrinol Metab 101:1606-1614.

366. Milham SJ, Elledge W 1972 Maternal methimazole and congenital defects in children. Teratology 5:125-126.

367. Clementi M, Di Gianantonio E, Cassina M, Leoncini E, Botto LD, Mastroiacovo P, SAFE-Med Study Group 2010 Treatment of hyperthyroidism in pregnancy and birth defects. J Clin Endocrinol Metab 95:E337-E341.

368. Foulds N, Walpole I, Elmslie F, Mansour S 2005 Carbimazole embryopathy: an emerging phenotype. Am J Med Genet A 132A(2):130-135.

369. Yoshihara A, Noh J, Yamaguchi T, Ohye H, Sato S, Sekiya K, Kosuga Y, Suzuki M, Matsumoto M, Kunii Y, Watanabe N, Mukasa K, Ito K, Ito K 2012. Treatment of Graves' disease with antithyroid drugs in the first trimester of pregnancy and the prevalence of congenital malformation. J Clin Endocrinol Metab 97:2396-2403.

370. Andersen SL, Olsen J, Wu CS, Laurberg P 2013 Birth defects after early pregnancy use of antithyroid drugs: a Danish nationwide study. J Clin Endocrinol Metab 98:4373-4381.

371. Andersen SL, Laurberg P 2014 Antithyroid drugs and congenital heart defects: ventricular septal defect is part of the methimazole/carbimazole embryopathy. Eur J Endocrinol 171(5):C1-C3.

372. Laurberg P, Andersen SL 2014 Therapy of endocrine disease: antithyroid drug use in early pregnancy and birth defects: time windows of relative safety and high risk? Eur J Endocrinol 171:R13-R20.

373. Andersen SL, Olsen J, Wu CS, Laurberg P 2014 Severity of birth defects after propylthiouracil exposure in early pregnancy. Thyroid 24:1533-1540.

374. Rubin PC 1981 Current concepts: beta-blockers in pregnancy. N Engl J Med 305:1323-1326.

375. Sherif IH, Oyan WT, Bosairi S, Carrascal SM 1991 Treatment of hyperthyroidism in pregnancy. Acta Obstet Gynecol Scand 70:461-463.

376. Nedrebo BG, Holm PI, Uhlving S, Sorheim JI, Skeie S, Eide GE, Husebye ES, Lien EA, Aanderud S 2002 Predictors of outcome and comparison of different drug regimens for the prevention of relapse in patients with Graves' disease. Eur J Endocrinol 147:583-589.

377. Moore KL, Persaud TVN, Torchia MG 2013 Human birth defects. In: The Developing Human: Clinically Oriented
Embryology. 9th edition. Saunders/Elsevier, Philadelphia, PA, pp 471-501.

378. Laurberg P, Krejbjerg A, Andersen SL 2014 Relapse following antithyroid drug therapy for Graves' hyperthyroidism. Curr Opin Endocrinol Diabetes Obes 21:415421.

379. Plummer HS 1923 Results of administering iodine to patients having exophthalmic goiter. JAMA 1923;80:155156.

380. Means JH 1948 The response to iodine and to antithyroid drugs in Graves' disease. In: The Thyroid and its Diseases. 2nd edition. Lippincott Company, Philadelphia, PA, pp 341-374.

381. Momotani N, Hisaoka T, Noh J, Ishikawa N, Ito K 1992 Effects of iodine on thyroid status of fetus versus mother in treatment of Graves' disease complicated by pregnancy. J Clin Endocrinol Metab 75:738-744.

382. Yoshihara A, Noh JY, Watanabe N, Mukasa K, Ohye H, Suzuki M, Matsumoto M, Kunii Y, Suzuki N, Kameda T, Iwaku K, Kobayashi S, Sugino K, Ito K 2015 Substituting potassium iodide for methimazole as the treatment for Graves' disease during the first trimester may reduce the incidence of congenital anomalies: a retrospective study at a single medical institution in Japan. Thyroid 25:11551161.

383. Connelly KJ, Boston BA, Pearce EN, Sesser D, Snyder D, Braverman LE, Pino S, LaFranchi SH 2012 Congenital hypothyroidism caused by excess prenatal maternal iodine ingestion. J Pediatr 161:760-762.

384. Chanoine JP, Pardou A, Bourdoux P, Delange F 1988 Withdrawal of iodinated disinfectants at delivery decreases the recall rate at neonatal screening for congenital hypothyroidism. Arch Dis Child 63:1297-1298.

385. Solomon BL, Wartofsky L, Burman KD 1993 Adjunctive cholestyramine therapy for thyrotoxicosis. Clin Endocrinol (Oxf) 38:39-43.

386. Mercado M, Mendoza-Zubieta V, Bautista-Osorio R, Espinoza-de los Monteros AL 1996 Treatment of hyperthyroidism with a combination of methimazole and cholestyramine. J Clin Endocrinol Metab 81:3191-3193.

387. Tsai WC, Pei D, Wang TF, Wu DA, Li JC, Wei CL, Lee $\mathrm{CH}$, Chen SP, Kuo SW 2005 The effect of combination therapy with propylthiouracil and cholestyramine in the treatment of Graves' hyperthyroidism. Clin Endocrinol (Oxf) 62:521-524.

388. Gurung V, Middleton P, Milan SJ, Hague W, Thornton JG 2013 Interventions for treating cholestasis in pregnancy. Cochrane Database Syst Rev 6:CD000493.

389. Leung AM, Pearce EN, Braverman LE 2010 Perchlorate, iodine and the thyroid. Best Pract Res Clin Endocrinol Metab 24:133-141.

390. Wenzel KW, Lente JR 1984 Similar effects of thionamide drugs and perchlorate on thyroid-stimulating immunoglobulins in Graves' disease: evidence against an immunosuppressive action of thionamide drugs. J Clin Endocrinol Metab 58:62-69.

391. EFSA CONTAM Panel (EFSA Panel on Contaminants in the Food Chain) 2014 Scientific Opinion on the risks to public health related to the presence of perchlorate in food, in particular fruits and vegetables. EFSA Journal 12(10):3869. Available at https://www.efsa.europa.eu/en/ efsajournal/pub/3869

392. Lazarus JH 2009 Lithium and thyroid. Best Pract Res Clin Endocrinol Metab 23:723-733. 
393. Diav-Citrin O, Shechtman S, Tahover E, Finkel-Pekarsky V, Arnon J, Kennedy D, Erebara A, Einarson A, Ornoy A 2014 Pregnancy outcome following in utero exposure to lithium: a prospective, comparative, observational study. Am J Psychiatry 171:785-794.

394. Donnelly MA, Wood C, Casey B, Hobbins J, Barbour LA 2015 Early severe fetal Graves disease in a mother after thyroid ablation and thyroidectomy. Obstet Gynecol 125:1059-1062.

395. Momotani N, Noh JY, Ishikawa N, Ito K 1997 Effects of propylthiouracil and methimazole on fetal thyroid status in mothers with Graves' hyperthyroidism. J Clin Endocrinol Metab 82:3633-3636.

396. Patil-Sisodia K, Mestman JH 2010 Graves hyperthyroidism and pregnancy: a clinical update. Endocr Pract 16:118-129.

397. Glinoer D 1998 Thyroid hyperfunction during pregnancy. Thyroid 8:859-864.

398. Momotani N, Noh J, Oyanagi H, Ishikawa N, Ito K 1986 Antithyroid drug therapy for Graves' disease during pregnancy. Optimal regimen for fetal thyroid status. $\mathrm{N}$ Engl J Med 315:24-28.

399. Bliddal S, Rasmussen AK, Sundberg K, Brocks V, FeldtRasmussen U 2011 Antithyroid drug-induced fetal goitrous hypothyroidism. Nat Rev Endocrinol 7:396-406.

400. Amino N, Tanizawa O, Mori H, Iwatani Y, Yamada T, Kurachi K, Kumahara Y, Miyai K 1982 Aggravation of thyrotoxicosis in early pregnancy and after delivery in Graves' disease. J Clin Endocrinol Metab 55:108-112.

401. Andersen SL, Olsen J, Carle A, Laurberg P 2015 Hyperthyroidism incidence fluctuates widely in and around pregnancy and is at variance with some other autoimmune diseases: a Danish population-based study. J Clin Endocrinol Metab 100:1164-1171.

402. Hamburger JI 1992 Diagnosis and management of Graves' disease in pregnancy. Thyroid 2:219-224.

403. Laurberg P, Bournaud C, Karmisholt J, Orgiazzi J 2009 Management of Graves' hyperthyroidism in pregnancy: focus on both maternal and foetal thyroid function, and caution against surgical thyroidectomy in pregnancy. Eur J Endocrinol 160:1-8.

404. Rotondi M, Cappelli C, Pirali B, Pirola I, Magri F, Fonte R, Castellano M, Rosei EA, Chiovato L 2008 The effect of pregnancy on subsequent relapse from Graves' disease after a successful course of antithyroid drug therapy. J Clin Endocrinol Metab 93:3985-3988.

405. Nakagawa Y, Mori K, Hoshikawa S, Yamamoto M, Ito S, Yoshida K 2002 Postpartum recurrence of Graves' hyperthyroidism can be prevented by the continuation of antithyroid drugs during pregnancy. Clin Endocrinol (Oxf) 57:467-471.

406. Abraham P, Avenell A, McGeoch SC, Clark LF, Bevan JS 2010 Antithyroid drug regimen for treating Graves' hyperthyroidism. Cochrane Database Syst Rev 20(1):CD003420.

407. McNab T, Ginsberg J 2005 Use of anti-thyroid drugs in euthyroid pregnant women with previous Graves' disease. Clin Invest Med 28:127-131.

408. The American College of Obstetricians and Gynecologists Committee Opinion on Nonobstetric Surgery During Pregnancy. Available at http://www.acog.org/ResourcesAnd-Publications/Committee-Opinions/Committee-onObstetric-Practice/Nonobstetric-Surgery-DuringPregnancy (accessed May 29, 2016).
409. Kempers MJ, van Tijn DA, van Trotsenburg AS, de Vijlder JJ, Wiedijk BM, Vulsma T 2003 Central congenital hypothyroidism due to gestational hyperthyroidism: detection where prevention failed. J Clin Endocrinol Metab 88:5851-5857.

410. Zwaveling-Soonawala N, van Trotsenburg P, Vulsma T 2009 Central hypothyroidism in an infant born to an adequately treated mother with Graves' disease: an effect of maternally derived thyrotrophin receptor antibodies? Thyroid 19:661-662.

411. Luton D, Le Gac I, Vuillard E, Castanet M, Guibourdenche J, Noel M, Toubert ME, Leger J, Boissinot C, Schlageter MH, Garel C, Tebeka B, Oury JF, Czernichow P, Polak M 2005 Management of Graves' disease during pregnancy: the key role of fetal thyroid gland monitoring. J Clin Endocrinol Metab 90:6093-6098.

412. McKenzie JM, Zakarija M 1992 Fetal and neonatal hyperthyroidism and hypothyroidism due to maternal TSH receptor antibodies. Thyroid 2:155-159.

413. Mitsuda N, Tamaki H, Amino N, Hosono T, Miyai K, Tanizawa O 1992 Risk factors for developmental disorders in infants born to women with Graves disease. Obstet Gynecol 80:359-364.

414. Peleg D, Cada S, Peleg A, Ben-Ami M 2002 The relationship between maternal serum thyroid-stimulating immunoglobulin and fetal and neonatal thyrotoxicosis. Obstet Gynecol 99:1040-1043.

415. Laurberg P, Wallin G, Tallstedt L, Abraham-Nordling M, Lundell G, Tørring O 2008 TSH-receptor autoimmunity in Graves' disease after therapy with anti-thyroid drugs, surgery, or radioiodine: a 5-year prospective randomized study. Eur J Endocrinol 158:69-75.

416. Laurberg P, Nygaard B, Glinoer D, Grussendorf M, Orgiazzi J 1998 Guidelines for TSH-receptor antibody measurements in pregnancy: results of an evidence-based symposium organized by the European Thyroid Association. Eur J Endocrinol 139:584-586.

417. Zimmerman D 1999 Fetal and neonatal hyperthyroidism. Thyroid 9:727-733.

418. Abeillon-du Payrat J, Chikh K, Bossard N, Bretones P, Gaucherand P, Claris O, Charrié A, Raverot V, Orgiazzi J, Borson-Chazot F, Bournaud C 2014 Predictive value of maternal second-generation thyroid-binding inhibitory immunoglobulin assay for neonatal autoimmune hyperthyroidism. Eur J Endocrinol 171:451-460.

419. Besancon A, Beltrand J, Le Gac I, Luton D, Polak M 2014 Management of neonates born to women with Graves' disease: a cohort study. Eur J Endocrinol 170:855-862.

420. Phoojaroenchanachai M, Sriussadaporn S, Peerapatdit T, Vannasaeng S, Nitiyanant W, Boonnamsiri V, Vichayanrat A 2001 Effect of maternal hyperthyroidism during late pregnancy on the risk of neonatal low birth weight. Clin Endocrinol (Oxf) 54:365-370.

421. Polak M, Le Gac I, Vuillard E, Guibourdenche J, Leger J, Toubert ME, Madec AM, Oury JF, Czernichow P, Luton D 2004 Fetal and neonatal thyroid function in relation to maternal Graves' disease. Best Pract Res Clin Endocrinol Metab 18:289-302.

422. Cohen O, Pinhas-Hamiel O, Sivan E, Dolitski M, Lipitz S, Achiron R 2003 Serial in utero ultrasonographic measurements of the fetal thyroid: a new complementary tool in the management of maternal hyperthyroidism in pregnancy. Prenat Diagn 23:740-742. 
423. Huel C, Guibourdenche J, Vuillard E, Ouahba J, Piketty M, Oury JF, Luton D 2009 Use of ultrasound to distinguish between fetal hyperthyroidism and hypothyroidism on discovery of a goiter. Ultrasound Obstet Gynecol 33:412-420.

424. Porreco RP, Bloch CA 1990 Fetal blood sampling in the management of intrauterine thyrotoxicosis. Obstet Gynecol 76:509-512.

425. Daffos F, Capella-Pavlovsky M, Forestier F 1985 Fetal blood sampling during pregnancy with use of a needle guided by ultrasound: a study of 606 consecutive cases. Am J Obstet Gynecol 153:655-660.

426. Nachum Z, Rakover Y, Weiner E, Shalev E 2003 Graves' disease in pregnancy: prospective evaluation of a selective invasive treatment protocol. Am J Obstet Gynecol 189:159-165.

427. Kilpatrick S 2003 Umbilical blood sampling in women with thyroid disease in pregnancy: is it necessary? Am J Obstet Gynecol 189:1-2.

428. Kahaly GJ 2015 Bioassays for TSH receptor antibodies: quo vadis? Eur Thyroid J 4:3-5.

429. Laurberg P, Pedersen KM, Vestergaard H, Sigurdsson G 1991 High incidence of multinodular toxic goitre in the elderly population in a low iodine intake area vs. high incidence of Graves' disease in the young in a high iodine intake area: comparative surveys of thyrotoxicosis epidemiology in East-Jutland Denmark and Iceland. J Intern Med 229:415-420.

430. Carle A, Pedersen IB, Knudsen N, Perrild H, Ovesen L, Rasmussen LB, Laurberg P 2011 Epidemiology of subtypes of hyperthyroidism in Denmark: a population-based study. Eur J Endocrinol 164:801-809.

431. Stagnaro-Green A 2012 Approach to the patient with postpartum thyroiditis. J Clin Endocrinol Meta 97:334-342.

432. Lazarus JH, Parkes AB, Premawardhana LD 2002 Postpartum thyroiditis. Autoimmunity 35:169-173.

433. Stagnaro-Green A 2004 Postpartum thyroiditis. Best Pract Res Clin Endocrinol Metab 18:303-316.

434. Azizi F 2006 Treatment of post-partum thyrotoxicosis. J Endocrinol Invest 29:244-247.

435. Struve CW, Haupt S, Ohlen S 1993 Influence of frequency of previous pregnancies on the prevalence of thyroid nodules in women without clinical evidence of thyroid disease. Thyroid 3:7-9.

436. Kung AW, Chau MT, Lao TT, Tam SC, Low LC 2002 The effect of pregnancy on thyroid nodule formation. $\mathrm{J}$ Clin Endocrinol Metab 87:1010-1014.

437. Tan GH, Gharib H, Goellner JR, van Heerden JA, Bahn RS 1996 Management of thyroid nodules in pregnancy. Arch Intern Med 156:2317-2320.

438. Marley EF, Oertel YC 1997 Fine-needle aspiration of thyroid lesions in 57 pregnant and postpartum women. Diagn Cytopathol 16:122-125.

439. Rosen IB, Walfish PG, Nikore V 1985 Pregnancy and surgical thyroid disease. Surgery 98:1135-1140.

440. Smith LH, Danielsen B, Allen ME, Cress R 2003 Cancer associated with obstetric delivery: results of linkage with the California cancer registry. Am J Obstet Gynecol 189:1128-1135.

441. Loh KC 1997 Familial nonmedullary thyroid carcinoma: a meta-review of case series. Thyroid 7:107-113.

442. Hegedus L 2004 Clinical practice. The thyroid nodule. N Engl J Med 351:1764-1771.

443. Haugen BR, Alexander EK, Bible KC, Doherty GM, Mandel SJ, Nikiforov YE, Pacini F, Randolph GW, Sawka
AM, Schlumberger M, Schuff KG, Sherman SI, Sosa JA, Steward DL, Tuttle RM, Wartofsky L 20162015 American Thyroid Association management guidelines for adult patients with thyroid nodules and differentiated thyroid cancer: the American Thyroid Association Guidelines Task Force on thyroid nodules and differentiated thyroid cancer. Thyroid 26:1-133.

444. Tucker MA, Jones PH, Boice JD Jr, Robison LL, Stone BJ, Stovall M, Jenkin RD, Lubin JH, Baum ES, Siegel SE 1991 Therapeutic radiation at a young age is linked to secondary thyroid cancer. The Late Effects Study Group. Cancer Res 51:2885-2888.

445. Pacini F, Vorontsova T, Demidchik E, Molinaro E, Agate, L, Romei C, Shavrova E, Cherstvoy ED, Ivashkevitch Y, Kuchinskaya E, Schlumberger M, Ronga G, Filesi M, Pinchera A 1997 Post-Chernobyl thyroid carcinoma in Belarus children and adolescents: comparison with naturally occurring thyroid carcinoma in Italy and France. J Clin Endocrinol Metab 81:3563-3569.

446. Tan GH, Gharib H, Reading CC 1995 Solitary thyroid nodule. Comparison between palpation and ultrasonography. Arch Intern Med 155:2418-2423.

447. Horvath E, Majlis S, Rossi R, Franco C, Niedmann JP, Castro A, Dominguez M 2009 An ultrasonogram reporting system for thyroid nodules stratifying cancer risk for clinical management. J Clin Endocrinol Metab 94:17481751.

448. Russ G, Royer B, Bigorgne C, Rouxel A, BienvenuPerrard M, Leenhardt L 2013 Prospective evaluation of thyroid imaging reporting and data system on 4550 nodules with and without elastography. Eur $\mathrm{J}$ Endocrinol 168:649-655.

449. Bennedbaek FN, Perrild H, Hegedus L 1999 Diagnosis and treatment of the solitary thyroid nodule. Results of a European survey. Clin Endocrinol (Oxf) 50:357-363.

450. Bennedbaek FN, Hegedus L 2000 Management of the solitary thyroid nodule: results of a North American survey. J Clin Endocrinol Metab 85:2493-2498.

451. Hegedus L, Bonnema SJ, Bennedbaek FN 2003 Management of simple nodular goiter: current status and future perspectives. Endocr Rev 24:102-132.

452. Costante G, Meringolo D, Durante C, Bianchi D, Nocera M, Tumino S, Crocetti U, Attard M, Maranghi M, Torlontano M, Filetti S 2007 Predictive value of serum calcitonin levels for preoperative diagnosis of medullary thyroid carcinoma in a cohort of 5817 consecutive patients with thyroid nodules. J Clin Endocrinol Metab 92:450455.

453. Pacini F, Pinchera A, Giani C, Grasso L, Baschieri L 1980 Serum thyroglobulin concentrations and 131I whole body scans in the diagnosis of metastases from differentiated thyroid carcinoma (after thyroidectomy). Clin Endocrinol (Oxf) 13:107-110.

454. Papini E, Guglielmi R, Bianchini A, Crescenzi A, Taccogna S, Nardi F, Panunzi C, Rinaldi R, Toscano V, Pacella CM 2002 Risk of malignancy in nonpalpable thyroid nodules: predictive value of ultrasound and color-Doppler features. J Clin Endocrinol Metab 87:1941-1946.

455. Belfiore A, La Rosa GL 2001 Fine-needle aspiration biopsy of the thyroid. Endocrinol Metab Clin North Am 30:361-400.

456. Goellner JR, Gharib H, Grant CS, Johnson DA 1987 Fine needle aspiration cytology of the thyroid, 1980 to 1986. Acta Cytol 31:587-590. 
457. Atkinson BF 1993 Fine needle aspiration of the thyroid. Monogr Pathol (35):166-199.

458. Solomon D 1993 Fine needle aspiration of the thyroid: an update. Thyroid Today 16:1-9.

459. Oertel YC 1996 Fine-needle aspiration and the diagnosis of thyroid cancer. Endocrinol Metab Clin North Am 25:69-91.

460. Singer PA 1996 Evaluation and management of the solitary thyroid nodule. Otolaryngol Clin North Am 29:577-591.

461. Powers CN, Frable WJ 1996 Fine Needle Aspiration Biopsy of the Head and Neck. Butterworth-Heinemann, Boston, MA.

462. Choe W, McDougall IR 1994 Thyroid cancer in pregnant women: diagnostic and therapeutic management. Thyroid 4:433-435.

463. Hamburger JI 1992 Thyroid nodules in pregnancy. Thyroid 2:165-168.

464. Herzon FS, Morris DM, Segal MN, Rauch G, Parnell T 1994 Coexistent thyroid cancer and pregnancy. Arch Otolaryngol Head Neck Surg 120:1191-1193.

465. Moosa M, Mazzaferri EL 1997 Outcome of differentiated thyroid cancer diagnosed in pregnant women. J Clin Endocrinol Metab 82:2862-2866.

466. Yasmeen S, Cress R, Romano PS, Xing G, Berger-Chen S, Danielsen B, Smith LH 2005 Thyroid cancer in pregnancy. Int J Gynaecol Obstet 91:15-20.

467. Gorman CA 1999 Radioiodine and pregnancy. Thyroid 9:721-726.

468. IAEA Radiation Protection of Patients [International Atomic Energy Agency] 2015 Pregnancy and Radiation Protection in Nuclear Medicine. Available at https://rpop .iaea.org/RPOP/RPoP/Content/SpecialGroups/

1_PregnantWomen/PregnancyNuclearMedicine.htm (accessed August 7, 2015).

469. Pauwels EK, Thomson WH, Blokland JA, Schmidt ME, Bourguignon M, El-Maghraby TA, Broerse JJ, Harding LK 1999 Aspects of fetal thyroid dose following iodine131 administration during early stages of pregnancy in patients suffering from benign thyroid disorders. Eur $\mathrm{J}$ Nucl Med 26:1453-1457.

470. Berg GE, Nystrom EH, Jacobsson L, Lindberg S, Lindstedt RG, Mattsson S, Niklasson CA, Noren AH, Westphal OG 1998 Radioiodine treatment of hyperthyroidism in a pregnant women. J Nucl Med 39:357-361.

471. Zanzonico PB 1997 Radiation dose to patients and relatives incident to 131I therapy. Thyroid 7:199-204.

472. Vannucchi G, Perrino M, Rossi S, Colombo C, Vicentini L, Dazzi D, Beck-Peccoz P, Fugazzola L 2010 Clinical and molecular features of differentiated thyroid cancer diagnosed during pregnancy. Eur J Endocrinol 162:145-151.

473. Lee JC, Zhao JT, Clifton-Bligh RJ, Gill AJ, Gundara JS, Ip J, Sywak MS, Delbridge LW, Robinson BG, Sidhu SB 2012 Papillary thyroid carcinoma in pregnancy: a variant of the disease? Ann Surg Oncol 19:4210-4216.

474. Messuti I, Corvisieri S, Bardesono F, Rapa I, Giorcelli J, Pellerito R, Volante M, Orlandi F 2014 Impact of pregnancy on prognosis of differentiated thyroid cancer: clinical and molecular features. Eur J Endocrinol 170:659-666.

475. Gharib H, Papini E, Valcavi R, Baskin HJ, Crescenzi A, Dottorini ME, Duick DS, Guglielmi R, Hamilton CR Jr, Zeiger MA, Zini M, AACE/AME Task Force on Thyroid Nodules 2006 American Association of Clinical Endocrinologists and Associazione Medici Endocrinologi medical guidelines for clinical practice for the diagnosis and management of thyroid nodules. Endocr Pract 12:63-102.
476. Rosen IB, Walfish PG 1986 Pregnancy as a predisposing factor in thyroid neoplasia. Arch Surg 121:1287-1290.

477. Doherty CM, Shindo ML, Rice DH, Montero M, Mestman JH 1995 Management of thyroid nodules during pregnancy. Laryngoscope 105:251-255.

478. Chong KM, Tsai YL, Chuang J, Hwang JL, Chen KT 2007 Thyroid cancer in pregnancy: a report of 3 cases. J Reprod Med 52:416-418.

479. Kuy S, Roman SA, Desai R, Sosa JA 2009 Outcomes following thyroid and parathyroid surgery in pregnant women. Arch Surg 144:399-406.

480. Uruno T, Shibuya H, Kitagawa W, Nagahama M, Suino K Ito K 2014 Optimal timing of surgery for differentiated thyroid cancer in pregnant women. World J Surg 38:704-708.

481. Mestman JH, Goodwin TM, Montoro MM 1995 Thyroid disorders of pregnancy. Endocrinol Metab Clin North Am 24:41-71.

482. American Thyroid Association (ATA) Guidelines Taskforce on Thyroid Nodules and Differentiated Thyroid Cancer, Cooper DS, Doherty GM, Haugen BR, Kloos RT, Lee SL, Mandel SJ, Mazzaferri EL, McIver B, Pacini F, Schlumberger M, Sherman SI, Steward DL, Tuttle RM 2009 Revised American Thyroid Association management guidelines for patients with thyroid nodules and differentiated thyroid cancer. Thyroid 19:1167-1214.

483. Wang CC, Friedman L, Kennedy GC, Wang H, Kebebew E, Steward DL, Zeiger MA, Westra WH, Wang Y, Khanafshar E, Fellegara G, Rosai J, Livolsi V, Lanman RB 2011 A large multicenter correlation study of thyroid nodule cytopathology and histopathology. Thyroid 21:243-251.

484. McLeod DS, Watters KF, Carpenter AD, Ladenson PW, Cooper DS, Ding EL 2012 Thyrotropin and thyroid cancer diagnosis: a systematic review and dose-response metaanalysis. J Clin Endocrinol Metab 97:2682-2692.

485. Pacini F, Schlumberger M, Dralle H, Elisei R, Smit JW, Wiersinga W, European Thyroid Cancer Taskforce 2006 European consensus for the management of patients with differentiated thyroid carcinoma of the follicular epithelium. Eur J Endocrinol 154:787-803.

486. Sawka AM, Lakra DC, Lea J, Alshehri B, Tsang RW, Brierley JD, Straus S, Thabane L, Gafni A, Ezzat S, George SR, Goldstein DP 2008 A systematic review examining the effects of therapeutic radioactive iodine on ovarian function and future pregnancy in female thyroid cancer survivors. Clin Endocrinol (Oxf) 69:479-490.

487. Garsi JP, Schlumberger M, Rubino C, Ricard M, Labbe M, Ceccarelli C, Schvartz C, Henri-Amar M, Bardet S, de Vathaire F 2008 Therapeutic administration of ${ }^{131}$ I for differentiated thyroid cancer: radiation dose to ovaries and outcome of pregnancies. J Nucl Med 49:845-852.

488. Pacini F, Gasperi M, Fugazzola L, Ceccarelli C, Lippi F, Centoni R, Martino E, Pinchera A 1994 Testicular function in patients with differentiated thyroid carcinoma treated with radioiodine. J Nucl Med 35:1418-1422.

489. FDA. Drug Approvals and Databases. Available at: www .fda.gov/Drugs/InformationonDrugs/default.htm (accessed February 11, 2017).

490. Rosvoll RV, Winship T 1965 Thyroid carcinoma and pregnancy. Surg Gynecol Obstet 121:1039-1042.

491. Hill CS, Jr, Clark RL, Wolf M 1966 The effect of subsequent pregnancy on patients with thyroid carcinoma. Surg Gynecol Obstet 122:1219-1222.

492. Leboeuf R, Emerick LE, Martorella AJ, Tuttle RM 2007 Impact of pregnancy on serum thyroglobulin and detection 
of recurrent disease shortly after delivery in thyroid cancer survivors. Thyroid 17:543-547.

493. Rosario PW, Barroso AL, Purisch S 2007 The effect of subsequent pregnancy on patients with thyroid carcinoma apparently free of the disease. Thyroid 17:1175-1176.

494. Hirsch D, Levy S, Tsvetov G, Weinstein R, Lifshitz A, Singer J, Shraga-Slutzky I, Grozinski-Glasberg S, Shimon I, Benbassat C 2010 Impact of pregnancy on outcome and prognosis of survivors of papillary thyroid cancer. Thyroid 20:1179-1185.

495. Ito Y, Miyauchi A, Kihara M, Higashiyama T, Kobayashi K, Miya A 2014 Patient age is significantly related to the progression of papillary microcarcinoma of the thyroid under observation. Thyroid 24:27-34.

496. Shindo H, Amino N, Ito Y, Kihara M, Kobayashi K, Miya A, Hirokawa M, Miyauchi A 2014 Papillary thyroid microcarcinoma might progress during pregnancy. Thyroid 24:840-844.

497. Wells SA Jr, Asa SL, Dralle H, Elisei R, Evans DB, Gagel RF, Lee N, Machens A, Moley JF, Pacini F, Raue F, Frank-Raue K, Robinson B, Rosenthal MS, Santoro M, Schlumberger M, Shah M, Waguespack SG, American Thyroid Association Guidelines Task Force on Medullary Thyroid Carcinoma 2015 Revised American Thyroid Association guidelines for the management of medullary thyroid carcinoma. Thyroid 25:567-610.

498. Bernal J, Pekonen F 1984 Ontogenesis of the nuclear 3,5,3'-triiodothyronine receptor in the human fetal brain. Endocrinology 114:677-679.

499. Thorpe-Beeston JG, Nicolaides KH, Felton CV, Butler J, McGregor AM 1991 Maturation of the secretion of thyroid hormone and thyroid-stimulating hormone in the fetus. N Engl J Med 324:532-536.

500. Thorpe-Beeston JG, Nicolaides KH, AM 1992 Fetal thyroid function. Thyroid 2:207-217.

501. Burrow GN, Fisher DA, Larsen PR 1994 Maternal and fetal thyroid function. N Engl J Med 331:1073-1078.

502. Brown RS 2004 Minireview: developmental regulation of thyrotropin receptor gene expression in the fetal and newborn thyroid. Endocrinology 145:4058-4061.

503. Ferreiro B, Bernal J, Goodyer CG, Branchard CL 1988 Estimation of nuclear thyroid hormone receptor saturation in human fetal brain and lung during early gestation. J Clin Endocrinol Metab 67:853-856.

504. Kester MH, Martinez de Mena R, Obregon MJ, Marinkovic D, Howatson A, Visser TJ, Hume R, Morreale de Escobar G 2004 Iodothyronine levels in the human developing brain: major regulatory roles of iodothyronine deiodinases in different areas. J Clin Endocrinol Metab 89:3117-3128.

505. Wu SY, Green WL, Huang WS, Hays MT, Chopra IJ 2005 Alternate pathways of thyroid hormone metabolism. Thyroid 15:943-958.

506. Ballabio M, Nicolini U, Jowett T, Ruiz de Elvira MC, Ekins RP, Rodeck CH 1989 Maturation of thyroid function in normal human fetuses. Clin Endocrinol (Oxf) 31:565-571.

507. Patel J, Landers K, Li H, Mortimer RH, Richard K 2011 Delivery of maternal thyroid hormones to the fetus. Trends Endocrinol Metab 22:164-170.

508. Vulsma T, Gons MH, de Vijlder JJ 1989 Maternal-fetal transfer of thyroxine in congenital hypothyroidism due to a total organification defect or thyroid agenesis. N Engl J Med 321:13-16.
509. Li H, Patel J, Mortimer RH, Richard K 2012 Ontogenic changes in human placental sodium iodide symporter expression. Placenta 33:946-948.

510. Mathieson L, Nielsen LK, Andersen JT, Grevys A, Sandlie I, Michaelsen TE, Hedegaard M, Knudsen LE, Dziegiel MH 2013 Maternofetal transplacental transport of recombinant IgG antibodies lacking effector functions. Blood 122:1174-1181.

511. Michalek K, Morshed SA, Latif R, Davies TF 2009 TSH receptor autoantibodies. Autimmun Rev 9:113-116.

512. Benvenga S, Ordookhani A, Pearce EN, Tonacchera M, Azizi F, Braverman LE 2008 Detection of circulating autoantibodies against thyroid hormones in an infant with permanent congenital hypothyroidism and her twin with transient congenital hypothyroidism: possible contribution of thyroid hormone autoantibodies to neonatal and infant hypothyroidism. J Pediatr Endocrinol Metab 21:1011-1020.

513. Zakarija M, McKenzie JM 1983 Pregnancy-associated changes in the thyroid-stimulating antibody of Graves' disease and the relationship to neonatal hyperthyroidism. J Clin Endocrinol Metab 57:1036-1040.

514. Skuza KA, Sills IN, Stene M, Rapaport R 1996 Prediction of neonatal hyperthyroidism in infants born to mothers with Graves disease. J Pediatr 128:264-268.

515. Brown RS, Bellisaro RL, Botero D, Fournier L, Abrams CA, Cowger ML, David R, Fort P, Richman RA 1996 Incidence of transient congenital hypothyroidism due to maternal thyrotropin receptor-blocking antibodies in over one million babies. J Clin Endocrinol Metab 81:11471151.

516. Fort P, Lifshitz F, Pugliese M, Klein I 1988 Neonatal thyroid disease: differential expression in three successive offspring. J Clin Endocrinol Metab 663:645-647.

517. Zakarija M, McKenzie JM, Munro DS 1983 Immunoglobulin $\mathrm{G}$ inhibitor of thyroid-stimulating antibody is a cause of delay in the onset of neonatal Graves' disease. J Clin Invest 72:1352-1356.

518. Kohn LD, Suzuki K, Hoffman WH, Tombaccini D, Marcocci C, Shimojo N, Watanabe Y, Amino N, Cho BY, Kohno Y, Hirai A, Tahara K 1997 Characterization of monoclonal thyroid-stimulating and thyrotropin bindinginhibiting autoantibodies from a Hashimoto's patient whose children had intrauterine and neonatal thyroid disease. J Clin Endocrinol Metab 82:3998-4009.

519. Matsuura N, Yamada Y, Nohara Y, Konishi J, Kasagi K, Endo K, Kojima H, Wataya K 1980 Familial neonatal transient hypothyroidism due to maternal TSH-binding inhibitor immunoglobulins. N Engl J Med 303:738-741.

520. Matsuura N, Konishi J 1990 Transient hypothyroidism in infants born to mothers with chronic thyroiditis-a nationwide study of twenty-three cases. The Transient Hypothyroidism Study Group. Endocrinol Jpn 37:369-379.

521. Ford G, LaFranchi SH 2014 Screening for congenital hypothyroidism: a worldwide view of strategies. Best Pract Res Clin Endocrinol Metab 28:175-187.

522. Rose SR, Brown RS, Foley T, Kaplowitz PB, Kaye CI, Sundararajan S, Varma SK 2006 Update of newborn screening and therapy for congenital hypothyroidism. Pediatrics 117:2290-2303.

523. Leger J, Olivieri A, Donaldson M, Torresani T, Krude H, van Vliet G, Polak M, Butler G, ESPE-PES-SLEP-JSPEAPEG-APPES-ISPAE, Congenital Hypothyroidism Consensus Conference Group 2014 European Society for Paediatric Endocrinology consensus guidelines on 
screening, diagnosis, and management of congenital hypothyroidism. J Clin Endocrinol Metab 99:363-384.

524. Carswell JM, Gordon JH, Popovsky E, Hale A, Brown RS 2013 Generic and brand-name L-thyroxine are not bioequivalent for children with severe congenital hypothyroidism. J Clin Endocrinol Metab 98:610-617.

525. LaFranchi SH 2011 Approach to the diagnosis and treatment of neonatal hypothyroidism. J Clin Endocrinol Metab 96:2959-2967.

526. Brown RS. Disorders of the thyroid gland in infants, children, and adolescents. In DeGroot LJ, ed. Thyroid Disease Manager. Available at http://www.thyroidmanager .org.

527. Buckshee K, Kriplani A, Kapil A, Bhargava VL, Takkar D 1992 Hypothyroidism complicating pregnancy. Aust N Z J Obstet Gynaecol 32:240-242.

528. Stein MT, Kessler DB, Hubbard E 2004 Failure to thrive in a four-month-old nursing infant. J Dev Behav Pediatr 25:S69-S73.

529. Hapon MB, Varas SM, Giménez MS, Jahn GA 2007 Reduction of mammary and liver lipogenesis and alteration of milk composition during lactation in rats by hypothyroidism. Thyroid 17:11-18.

530. Thrift TA, Bernal A, Lewis AW, Neuendorff DA, Willard CC, Randel RD 1999 Effects of induced hypothyroidism on weight gains, lactation, and reproductive performance of primiparous Brahman cows. J Anim Sci 77:1844-1850.

531. Rosato RR, Gimenez MS, Jahn GA 1992 Effects of chronic thyroid hormone administration on pregnancy, lactogenesis and lactation in the rat. Acta Endocrinol (Copenh) 127:547-554.

532. Varas SM, Muñoz EM, Hapon MB, Aguilera Merlo CI, Giménez MS, Jahn GA 2002 Hyperthyroidism and production of precocious involution in the mammary glands of lactating rats. Reproduction 124:691-702.

533. van Wassenaer AG, Stulp MR, Valianpour F, Tamminga P, Ris Stalpers C, de Randamie JS, van Beusekom C, de Vijlder JJ 2002 The quantity of thyroid hormone in human milk is too low to influence plasma thyroid hormone levels in the very preterm infant. Clin Endocrinol (Oxf) 56:621-627.

534. Akasha MA, Anderson RR, Ellersieck M, Nixon DA 1987 Concentration of thyroid hormones and prolactin in dairy cattle serum and milk at three stages of lactation. J Dairy Sci 70:271-276.

535. Akasha M, Anderson RR 1984 Thyroxine and triiodothyronine in milk of cows, goats, sheep, and guinea pigs. Proc Soc Exp Biol Med 177:360-371.

536. Todini L, Salimei E, Malfatti A, Ferraro S, Fantuz F 2012 Thyroid hormones in milk and blood of lactating donkeys as affected by stage of lactation and dietary supplementation with trace elements. J Dairy Res 79:232-237.

537. Howe DB, Beardsley M, Bakhsh S 2008 Appendix U. Model procedure for release of patients or human research subjects administered radioactive materials. In Consolidated Guidance About Materials Licenses. ProgramSpecific Guidance About Medical Use Licenses. Final Report. U.S. Nuclear Regulatory Commission Office of Nuclear Material Safety and Safeguards. NUREG-1556, vol. 9, rev. 2. Available at http://www.nrc.gov/reading-rm/ doc-collections/nuregs/staff/sr1556/v9/r2/.

538. Kampmann JP, Johansen K, Hansen JM, Helweg J 1980 Propylthiouracil in human milk. Revision of a dogma. Lancet 1:736-737.
539. Momotani N, Yamashita R, Yoshimoto M, Noh J, Ishikawa N, Ito K 1989 Recovery from foetal hypothyroidism: evidence for the safety of breast-feeding while taking propylthiouracil. Clin Endocrinol (Oxf) 31:591-595.

540. Momotani N, Yamashita R, Makino F, Noh JY, Ishikawa N, Ito K 2000 Thyroid function in wholly breast-feeding infants whose mothers take high doses of propylthiouracil. Clin Endocrinol (Oxf) 53:177-181.

541. Johansen K, Andersen AN, Kampmann JP, Mølholm Hansen JM, Mortensen HB 1982 Excretion of methimazole in human milk. Eur J Clin Pharmacol 23:339-341.

542. Cooper DS, Bode HH, Nath B, Saxe V, Maloof F, Ridgway EC 1984 Methimazole pharmacology in man: studies using a newly developed radioimmunoassay for methimazole. J Clin Endocrinol Metab 58:473-479.

543. Azizi F, Khoshniat M, Bahrainian M, Hedayati M 2000 Thyroid function and intellectual development of infants nursed by mothers taking methimazole. J Clin Endocrinol Metab 85:3233-3238.

544. Lamberg BA, Ikonen E, Osterlund K, Teramo K, Pekonen F, Peltola J, Välimäki M 1984 Antithyroid treatment of maternal hyperthyroidism during lactation. Clin Endocrinol (Oxf) 21:81-87.

545. Azizi F 1996 Effect of methimazole treatment of maternal thyrotoxicosis on thyroid function in breast-feeding infants. J Pediatr 128:855-858.

546. Institute of Medicine, Food and Nutrition Board 2001 Dietary Reference Intakes for Vitamin A, Vitamin K, Arsenic, Boron, Chromium, Copper, Iodine, Iron, Manganese, Molybdenum, Nickel, Silicon, Vanadium, and Zinc. National Academy Press, Washington, DC.

547. World Health Organization, UNICEF, ICCIDD 2007 Assessment of Iodine Deficiency Disorders and Monitoring Their Elimination. A Guide for Programme Managers. 3rd edition. Geneva, Switzerland.

548. Amino N, Mori H, Iwatani Y, Tanizawa O, Kawashima M, Tsuge I, Ibaragi K, Kumahara Y, Miyai K 1982 High prevalence of transient post-partum thyrotoxicosis and hypothyroidism. N Engl J Med 306:849-852.

549. Stagnaro-Green A, Schwartz A, Gismondi R, Tinelli A, Mangieri T, Negro R 2011 High rate of persistent hypothyroidism in a large-scale prospective study of postpartum thyroiditis in southern Italy. J Clin Endocrinol Metab 96:652-657.

550. Muller AF, Drexhage HA, Berghout A 2001 Postpartum thyroiditis and autoimmune thyroiditis in women of childbearing age: recent insights and consequences for antenatal and postnatal care. Endocr Rev 22:605-630.

551. Kuijpens JL, De Hann-Meulman M, Vader HL, Pop VJ, Wiersinga WM, Drexhage HA 1998 Cell-mediated immunity and postpartum thyroid dysfunction: a possibility for the prediction of disease? J Clin Endocrinol Metab 83:1959-1966.

552. Stagnaro-Green A, Roman SH, Cobin RH, el-Harazy E, Wallenstein S, Davies TF 1992 A prospective study of lymphocyte-initiated immunosuppression in normal pregnancy: evidence of a T-cell etiology for postpartum thyroid dysfunction. J Clin Endocrinol Metab 74:645-653.

553. Smallridge RC 2000 Postpartum thyroid disease: a model of immunologic dysfunction. Clin Appl Immunol Rev 1:89-103.

554. Ide A, Amino N, Kang S, Yoshioka W, Kudo T, Nishihara E, Ito M, Nakamura H, Miyauchi A 2014 Differentiation of postpartum Graves' thyrotoxicosis from postpartum 
destructive thyrotoxicosis using antithyrotropin receptor antibodies and thyroid blood flow. Thyroid 24:10271031.

555. Nicholson WK, Robinson KA, Smallridge RC, Ladenson PW, Powe NR 2006 Prevalence of postpartum thyroid dysfunction: a quantitative review. Thyroid 16:573-582.

556. Gerstein HC 1993 Incidence of postpartum thyroid dysfunction in patients with type I diabetes mellitus. Ann Intern Med 118:419-423.

557. Alvarez-Marfany M, Roman SH, Drexler AJ, Robertson C, Stagnaro-Green A 1994 Long-term prospective study of postpartum thyroid dysfunction in women with insulin dependent diabetes mellitus. J Clin Endocrinol Metab 79:10-16.

558. Elefsiniotis IS, Vezali E, Pantazis KD, Saroglou G 2008 Post-partum thyroiditis in women with chronic viral hepatitis. J Clin Virol 41:318-319.

559. Stagnaro-Green A, Akhter E, Yim C, Davies TF, Magder L, Petri M 2011 Thyroid disease in pregnant women with systemic lupus erythematosus: increased preterm delivery. Lupus 20:690-699.

560. Tagami T, Hagiwara H, Kimura T, Usui T, Shimatsu A, Naruse M 2007 The incidence of gestational hyperthyroidism and postpartum thyroiditis in treated patients with Graves' disease. Thyroid 17:767-772.

561. Manetti L, Parkes AB, Lupi I, Di Cianni G, Bogazzi F, Albertini S, Morselli LL, Raffaelli V, Russo D, Rossi G, Gasperi M, Lazarus JH, Martino E 2008 Serum pituitary antibodies in normal pregnancy and in patients with postpartum thyroiditis: a nested case-control study. Eur J Endocrinol 159:805-809.

562. Lazarus JH, Ammari F, Oretti R, Parkes AB, Richards CJ, Harris B 1997 Clinical aspects of recurrent postpartum thyroiditis. Br J Gen Pract 47:305-308.

563. Caixas A, Albareda M, Garcia-Patterson A, RodriguezEspinosa J, de Leiva A, Corcoy R 1999 Postpartum thyroiditis in women with hypothyroidism antedating pregnancy? J Clin Endocrinol Metab 84:4000-4005.

564. Marqusee E, Hill JA, Mandel SJ 1997 Thyroiditis after pregnancy loss. J Clin Endocrinol Metab 82:2455-2457.

565. Walfish PG, Meyerson J, Provias JP, Vargas MT, Papsin FR 1992 Prevalence and characteristics of post-partum thyroid dysfunction: results of a survey from Toronto, Canada. J Endocrinol Invest 15:265-272.

566. Hayslip CC, Fein HG, O’Donnell VM, Friedman DS, Klein TA, Smallridge RC 1988 The value of serum antimicrosomal antibody testing in screening for symptomatic postpartum thyroid dysfunction. Am J Obstet Gynecol 159:203-209.

567. Lazarus JH 1999 Clinical manifestations of postpartum thyroid disease. Thyroid 9:685-689.

568. Kuijpens JL, Vader HL, Drexhage HA, Wiersinga WM, van Son MJ, Pop VJ 2001 Thyroid peroxidase antibodies during gestation are a marker for subsequent depression postpartum. Eur J Endocrinol 145:579-584.

569. Pop VJ, de Rooy HA, Vader HL, van der Heide D, van Son M, Komproe IH, Essed GG, de Geus CA 1991 Postpartum thyroid dysfunction and depression in an unselected population. N Engl J Med 324:1815-1816.

570. Hayslip CC, Fein HG, O'Donnell VM, Friedman DS, Klein TA, Smallridge RC 1988 The value of serum antimicrosomal antibody testing in screening for symptomatic postpartum thyroid dysfunction. Am J Obstet Gynecol 159:203-209.
571. Lucas A, Pizarro E, Granada ML, Salinas I, Sanmarti A 2001 Postpartum thyroid dysfunction and postpartum depression: are they two linked disorders? Clin Endocrinol (Oxf) 55:809-814.

572. Lazarus JH, Hall R, Othman S, Parkes AB, Richards CJ, McCulloch B, Harris B 1996 The clinical spectrum of postpartum thyroid disease. QJM 89:429-435.

573. Le Donne M, Settineri S, Benvenga S 2012 Early pospartum alexithymia and risk for depression: relationship with serum thyrotropin, free thyroid hormones and thyroid autoantibodies. Psychoneuroendocrinology 37:519-533.

574. Harris B, Othman S, Davies JA, Weppner GJ, Richards CJ, Newcombe RG, Lazarus JH, Parkes AB, Hall R, Phillips DI 1992 Association between postpartum thyroid dysfunction and thyroid antibodies and depression. BMJ 305: $152-156$

575. Pop VJ, de Rooy HA, Vader HL, van der Heide D, van Son MM, Komproe IH 1993 Microsomal antibodies during gestation in relation to postpartum thyroid dysfunction and depression. Acta Endocrinol (Copenh) 129:26-30.

576. Harris B, Oretti R, Lazarus J, Parkes A, John R, Richards C, Newcombe R, Hall R 2002 Randomised trial of thyroxine to prevent postnatal depression in thyroidantibody-positive women. Br J Psychiatry 180:327-330.

577. Fung HY, Kologlu M, Collison K, John R, Richards CJ, Hall R, McGregor AM 1988 Postpartum thyroid dysfunction in Mid Glamorgan. Br Med J (Clin Res Ed) 296:241-244.

578. Vargas MT, Briones-Urbina R, Gladman D, Papsin FR, Walfish PG 1988 Antithyroid microsomal autoantibodies and HLA-DR5 are associated with postpartum thyroid dysfunction: evidence supporting an autoimmune pathogenesis. J Clin Endocrinol Metab 67:327-333.

579. Rasmussen NG, Hornnes PJ, Hoier-Madsen M, FeldtRasmussen U, Hegedus L 1990 Thyroid size and function in healthy pregnant women with thyroid autoantibodies. Relation to development of postpartum thyroiditis. Acta Endocrinol (Copenh) 123:395-401.

580. Azizi F 2005 The occurrence of permanent thyroid failure in patients with subclinical postpartum thyroiditis. Eur $\mathbf{J}$ Endocrinol 153:367-371.

581. Nohr SB, Jorgensen A, Pedersen KM, Laurberg P 2000 Postpartum thyroid dysfunction in pregnant thyroid peroxidase antibody-positive women living in an area with mild to moderate iodine deficiency: is iodine supplementation safe? J Clin Endocrinol Metab 85:3191-3198.

582. Kampe O, Jansson R, Karlsson FA 1990 Effects of Lthyroxine and iodide on the development of autoimmune postpartum thyroiditis. J Clin Endocrinol Metab 70:10141018.

583. Klein RZ, Haddow JE, Faix JD, Brown RS, Hermos RJ, Pulkkinen A, Mitchell ML 1991 Prevalence of thyroid deficiency in pregnant women. Clin Endocrinol (Oxf) 35:41-46.

584. Negro R, Schwartz A, Gismondi R, Tinelli A, Mangieri T, Stagnaro-Green A 2010 Universal screening versus case finding for detection and treatment of thyroid hormonal dysfunction during pregnancy. J Clin Endocrinol Metab 95:1699-1707.

585. Ma L, Qi H, Chai X, Jiang F, Mao S, Liu J, Zhang S, Lian X, Sun X, Wang D, Ren, Yan Q 2016 The effects of screening and intervention of subclinical hypothyroidism on pregnancy outcomes: a prospective multicenter singleblind, randomized, controlled study of thyroid function 
screening test during pregnancy. J Matern Fetal Neonatal Med 29:1391-1394.

586. TABLET trial: Randomised Controlled Trial of the Efficacy and Mechanism of Levothyroxine Treatment on Pregnancy and Neonatal Outcomes in Women with Thyroid Antibodies (TABLET). Available at http://www. birmingham.ac.uk/research/activity/mds/trials/bctu/trials/ womens/tablet/index.aspx.

587. Nederlands TRIAL Register. T4Life trial. http://www. trialregister.nl/trialreg/admin/rctview.asp?TC $=3364$.

588. Teng W, Shan Z 2011 Pregnancy and thyroid diseases in China. Thyroid 21:1053-1055.

589. Dosiou C, Sanders GD, Araki SS, Crapo LM 2008 Screening pregnant women for autoimmune thyroid disease: a costeffectiveness analysis. Eur J Endocrinol 158:841-851.

590. Dosiou C, Barnes J, Schwartz A, Negro R, Crapo L, Stagnaro-Green A 2012 Cost-effectiveness of universal and risk-based screening for autoimmune thyroid disease in pregnant women. J Clin Endocrinol Metab 97:1536-1546.

591. Thung SF, Funai EF, Grobman WA 2009 The costeffectiveness of universal screening in pregnancy for subclinical hypothyroidism. Am J Obstet Gynecol 200: 267.e1-267.e7.

592. Donnay Candil S, Balsa Barro JA, Álvarez Hernández J, Crespo Palomo C, Pérez-Alcántara F, Polanco Sánchez C 2015 Cost-effectiveness analysis of universal screening for thyroid disease in pregnant women in Spain. Endocrinol Nutrition 62:322-330.

593. Rowley PT, Loader S, Kaplan RM 1998 Prenatal screening for cystic fibrosis carriers: an economic evaluation. Am J Hum Genet 63:1160-1174.

594. Werner EF, Pettker CM, Zuckerwise L, Reel M, Funai EF, Henderson J, Thung SF 2012 Screening for gestational diabetes mellitus: are the criteria proposed by the International Association of the Diabetes and Pregnancy Study Groups cost-effective? Diabetes Care 35:529-535.

595. Potlukova E, Potluka O, Jiskra J, Limanova Z, Telicka Z, Bartakova J, Springer D 2012 Is age a risk factor for hypothyroidism in pregnancy? An analysis of 5223 pregnant women. J Clin Endocrinol Metab 97:1945-1952.

596. Yang H, Shao M, Chen L, Chen Q, Yu L, Cai L, Lin Z, Zhang C, Lu X 2014 Screening strategies for thyroid disorders in the first and second trimester of pregnancy in China. PLoS ONE 9(6):e99611.

597. Medeiros MF, Cerqueira TL, Silva Junior JC, Amaral MT, Vaidya B, Poppe KG, Carvalho GA, Gutierrez S, Alcaraz G, Abalovich M, Ramos HE, Latin American Thyroid Society 2014 An international survey of screeningand management of hypothyroidismduring pregnancy in Latin America. Arq Bras Endocrinol Metab 58:906-911.

598. Vaidya B, Hubalewska-Dydejczyk A, Laurberg P, Negro R, Vermiglio F, Poppe K 2012 Treatment and screening of hypothyroidism in pregnancy: results of a European survey. Eur J Endocrinol 166:49-54.

599. Azizi F, Amouzegar A, Mehran L, Alamdari S, Subekti I, Vaidya B, Poppe K, San Luis T Jr, Akamizu T 2014 Screening and management of hypothyroidism in pregnancy: results of an Asian Survey. Endocr J 61:697-704.

600. Srimatkandada P, Stagnaro-Green A, Pearce EN 2015 Attitudes of ATA survey respondents toward screening and treatment of hypothyroidism in pregnancy. Thyroid 25:368-369.

601. Lazarus J, Brown RS, Daumerie C, HubalewskaDydejczyk A, Negro R, Vaidya B 20142014 European
Thyroid Association Guidelines for the management of subclinical hypothyroidism in pregnancy and in children. Eur Thyroid J 3:76-94.

602. Vila L, Velasco I, González S, Morales F, Sánchez E, Lailla JM, Martinez-Astorquiza T, Puig-Domingo M, Grupo de Trabajo de Trastornos por Deficiencia de Yodo y Disfunción Tiroidea de la Sociedad Española de Endocrinología y Nutrición 2012 Detection of thyroid dysfunction in pregnant women: universal screening is justified. Endocrinol Nutr 59:547-60.

603. 2012 Indian Thyroid Society Guidelines for Management of Thyroid Dysfunction During Pregnancy. Clinical Practice Guidelines. Elsevier. New Delhi.

604. Maternal Health Division, Ministry of Health and Family Welfare, Government of India 2014 National guidelines for screening of hypothyroidism during pregnancy. Available at http://nrhm.gov.in/images/pdf/programmes/ maternal-health/guidelines/National_Guidelines_for_ Screening_of_Hypothyroidism_during_Pregnancy.pdf

605. Practice Committee of the American Society for Reproductive Medicine 2015 Subclinical hypothyroidism in the infertile female population: a guideline. Fertil and Steril 104:545-553.

606. Canaris GJ, Steiner JF, Ridgway EC 1997 Do traditional symptoms of hypothyroidism correlate with biochemical disease? J Gen Intern Med 12:544-550.

607. Canaris GJ, Manowitz NR, Mayor G, Ridgway EC 2000 The Colorado thyroid disease prevalence study. Arch Intern Med 160:526-534.

608. Jovanovic-Peterson L, Peterson CM 1988 De novo clinical hypothyroidism in pregnancies complicated by type I diabetes, subclinical hypothyroidism, and proteinuria: a new syndrome. Am J Obstet Gynecol 159:442-446.

609. Ladenson PW 2005 Diagnosis of hypothyroidism. In: Braverman LE, Utiger RD (eds) Werner \& Ingbar's The Thyroid: A Fundamental and Clinical Text. Ninth edition. Lippincott, Williams \& Wilkins, Philadelphia, PA, pp 857-863.

610. Mercado G, Adelstein DJ, Saxton JP, Secic M, Larto MA, Lavertu P 2001 Hypothyroidism: a frequent event after radiotherapy and after radiotherapy with chemotherapy for patients with head and neck carcinoma. Cancer 92:28922897.

611. De Carlucci D Jr, Tavares MR, Obara MT, Martins LA, Hojaij FC, Cernea CR 2008 Thyroid function after unilateral total lobectomy: risk factors for postoperative hypothyroidism. Arch Otolaryngol Head Neck Surg 134: 1076-1079.

612. Verloop H, Louwerens M, Schoones JW, Kievit J, Smit JW, Dekkers OM 2012 Risk of hypothyroidism following hemithyroidectomy: systematic review and meta-analysis of prognostic studies. J Clin Endocrinol Metab 97:22432255.

613. Strieder TG, Prummel MF, Tijssen JG, Endert E, Wiersinga WM 2003 Risk factors for and prevalence of thyroid disorders in a cross-sectional study among healthy female relatives of patients with autoimmune thyroid disease. Clin Endocrinol (Oxf) 59:396-401.

614. Manji N, Carr-Smith JD, Boelaert K, Allahabadia A, Armitage M, Chatterjee VK, Lazarus JH, Pearce SH, Vaidya B, Gough SC, Franklyn JA 2006 Influences of age, gender, smoking, and family history on autoimmune thyroid disease phenotype. J Clin Endocrinol Metab 91:4873-4880. 
615. Michalaki MA, Vagenakis AG, Leonardou AS, Argentou MN, Habeos IG, Makri MG, Psyrogiannis AI, Kalfarentzos FE, Kyriazopoulou VE 2006 Thyroid function in humans with morbid obesity. Thyroid 16:73-78.

616. Rotondi M, Leporati P, La Manna A, Pirali B, Mondello T, Fonte R, Magri F, Chiovato L 2009 Raised serum TSH levels in patients with morbid obesity: is it enough to diagnose subclinical hypothyroidism? Eur $\mathrm{J}$ Endocrinol 160:403-408.

617. Eskes SA, Wiersinga WM 2009 Amiodarone and thyroid. Best Pract Res Clin Endocrinol Metab 23:735-751.

618. Vagenakis AG, Braverman LE 1975 Adverse effects of iodides on thyroid function. Med Clin North Am 59: 1075-1088.

619. Carle A, Pedersen IB, Knudsen N, Perrild H, Ovesen L, Rasmussen LB, Laurberg P 2014 Development of autoimmune overt hypothyroidism is highly associated with live births and induced abortions but only in premenopausal women. J Clin Endocrinol Metab 99:2241-2249.

620. Zimmermann MB, Boelaert K 2015 Iodine deficiency and thyroid disorders. Lancet Diabetes Endocrinol 3:286-295.

621. Premawardhana LD, Parkes AB, John R, Harris B, Lazarus JH 2004 Thyroid peroxidase antibodies in early pregnancy: utility for prediction of postpartum thyroid dysfunction and implications for screening. Thyroid 14:610-615.

Address correspondence to: Elizabeth N. Pearce, MD, MSc

Section of Endocrinology, Diabetes, and Nutrition Boston University School of Medicine

88 East Newton St., H3600

Boston, MA 02118

E-mail: elizabeth.pearce@bmc.org 